TRANSFORMING EXPERIENCES:

\title{
A REFLECTIVE TOPICAL AUTOBIOGRAPHY OF FACILITATING STUDENT NURSE DEVELOPMENT THROUGH INTERNATIONAL IMMERSION PROGRAMMES
}

by

Mary Anne Levine

A thesis

submitted to the Victoria University of Wellington

in fulfilment of the requirements for the degree of

Doctor of Philosophy in Midwifery

Victoria University of Wellington

2006 


\begin{abstract}
The focus of this study is the impact of an international education programme on baccalaureate nursing students taken by me, their teacher/professor, to become immersed in another culture. This is an unusual undertaking for a nursing education programme but it is one to which I have been passionately committed for more than 20 years. This study examines my life-work in a deeply reflective and narrative way. I have used many sources of data to assist in the creation of my story including the framework of Moustakas (1990) and Reflective Topical Autobiography as described by Johnstone (1999). My story is woven throughout this thesis as I gradually reveal more of myself as I feel that who I am should be a continuous thread that lends credence to multiple sections of this work. Several of my reflective stories about the immersion programme experiences, called here, vignettes are included, so that my reflections, thoughts, and feelings can be expressed. "I didn't have to create the world I wrote about it. I realized that words could tell. [sic] That there was such a thing as an emotional sentence” (Lorde, 1984, p. 85). The genesis of the emotional sentence emerged through the use of interviews with student participants and my own introspective process. In this way I came to a new understanding of myself and my passion for this way of working.

I found that these educational experiences had the ability to change the personal and professional lives of participants. Students’ world views expanded exponentially as the true-life experiences in which they actively participated nurtured a profound metamorphosis. It is important to recognise the critical social nature of this work. I have carefully considered the issues of class and gender, poverty and powerlessness, and the inherent dialectic as key elements in the reflective process. The awareness of these social issues coupled with profound personal changes that occurred when immersed in another culture frame the contribution of this work to the profession of nursing in general and to midwifery specifically. In addition, I have been changed. My “way of being” has become radically different. I realise that I facilitate life transformation for participants by
\end{abstract}


providing the platform; I realise the connection, potency, and power of studentteacher relationships; and most of all I learned that I teach from the heart. 


\title{
DEDICATION
}

\author{
Dedicated to: \\ The Poor childbearing Women of the Developing World: \\ The Silent Majority! \\ Oh-for the touch of a vanished hand \\ or the sound of a voice now still \\ Anon \\ And \\ My daughter \\ Joey Shot With Two Arrows, \\ A casualty of colonialism
}

\author{
And \\ My daughter \\ Gita Belfer \\ A daughter of India \\ Por fin! Está hecho!
}

And

My sister Paula,

a forever support

And

My external thesis advisor,

Wendy Woodward, for her staunch support,

constructive criticism, and infinite patience

And

My parents, grandparents, myriad aunts, uncles, and cousins, who comprised the extraordinary extended family from whence I have come and who moulded me into who I am today

I am truly sorry that most of you are long since gone, yet live on! 
Marcos Katz

The APA Maven

Melinda Christensen, whiz of computer formatting

Kaia's Joseph

For super computer expertise

AND

Let me tell you the secret

That has led me

To my goal:

My strength lies solely in my tenacity.

Louis Pasteur 


\title{
ACKNOWLEDGEMENTS
}

\author{
Maralyn Foureur, primary thesis advisor, \\ for your continued gentle encouragement, lack of exasperation, and support \\ throughout this five year process
}

\begin{abstract}
Librarians:
Justin Cargill at Victoria University of Wellington, New Zealand and

Sharon Chadwick at Humboldt State University (HSU), Arcata, California: Although at disparate "ends of the earth" I offer my deepest appreciation for your commitment in enabling me to successfully navigate the world of books and data bases.
\end{abstract}

Julie Graham, distance librarian, HSU (who is probably more ecstatic than I, at my PhD completion), for her unstinting support in accessing the world's libraries

Giselle Rohde, serials manager, HSU library: Mein tiefster dank für die fachmännische hilfe mit Microform

Mary Kay, HSU library information services: For your computer expertise in accessing on-line documents

Diane Benson, fellow professor, HSU, for your endless help with End-Note

To Lori McClean, former nursing department secretary, HSU, for your kindness, responding to my computer logistic questions

Victoria University of Wellington

Jan Duke, Rose McEldowney, Abbey McDonald and Stuart Gates...thanks for your "always" availability

Bill Ehlert-Abler

Thanks for the many hours spent in the final reading of my manuscript and the linguistic errors that you detected 
ABSTRACT .........................................................................ii

DEDICATION ..............................................................................iv

ACKNOWLEDGEMENTS ...............................................................vi

TABLE OF CONTENTS ..........................................................vii

LIST OF TABLES........................................................................xi

CHAPTER I INITIAL ENGAGEMENT ........................................ 1

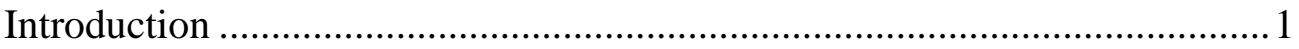

My Teaching Philosophy .................................................................... 2

Qualifications as Programme Director ..................................................... 4

Value of Immersion Programmes............................................................. 7

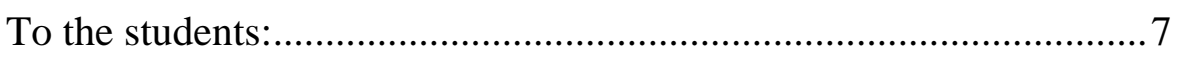

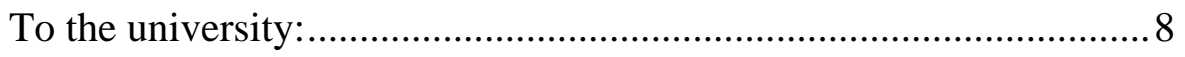

To the discipline of nursing: ......................................................... 8

To the hospitals and community health facilities:............................ 9

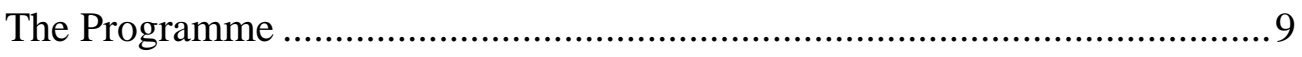

Study Abroad Programmes................................................................... 17

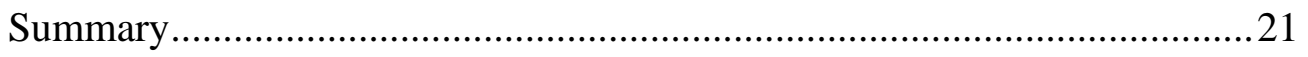

CHAPTER II IMMERSION .........................................................22

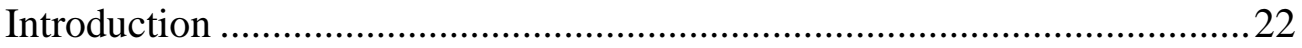

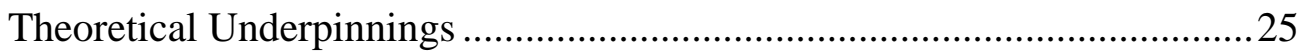

Reflective Topical Autobiography (RTA)......................................26

Autobiography/biography, and interpretive materials.......................33

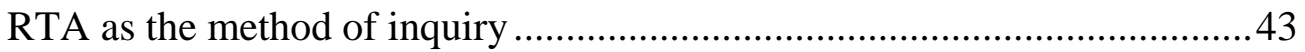

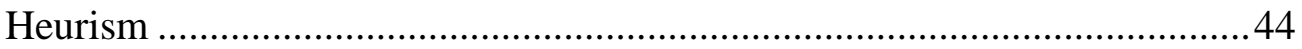

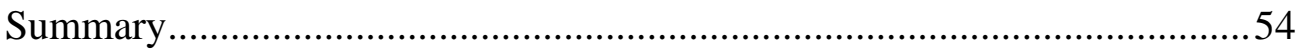

CHAPTER III STUDY DESIGN ..................................................55

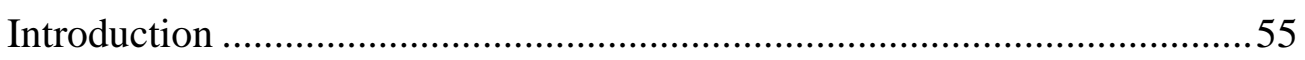

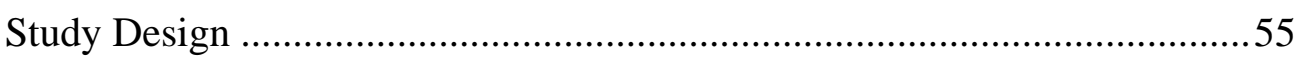




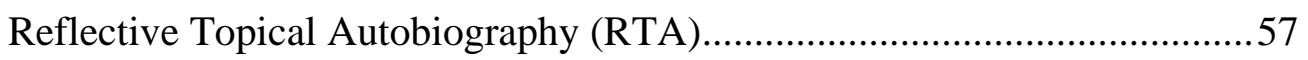

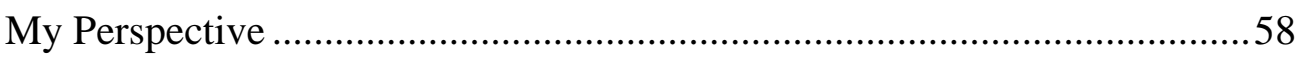

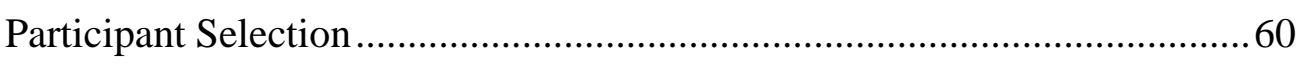

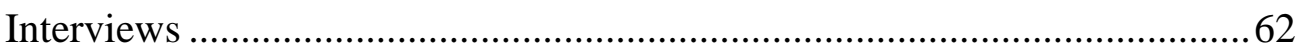

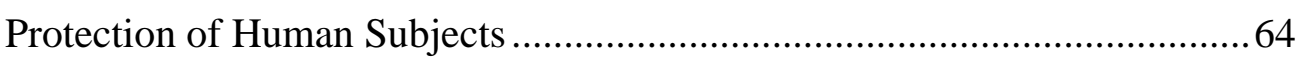

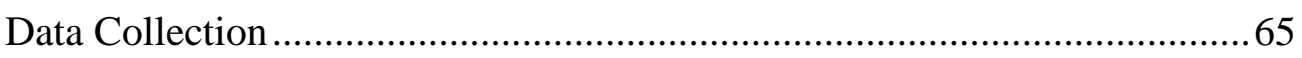

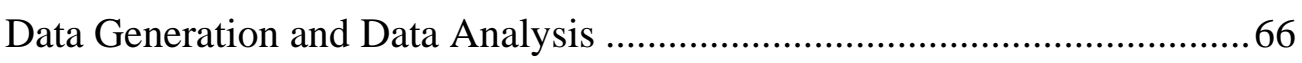

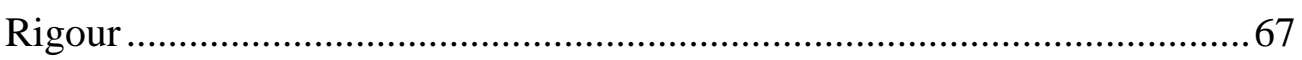

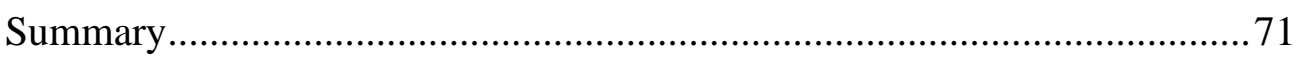

CHAPTER IV INCUBATION: THE REAL STORY ........................72

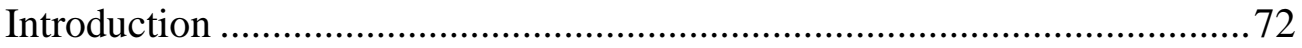

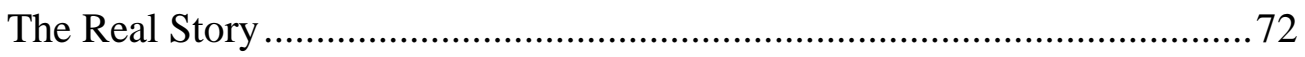

Development of Interest: My Story ………............................................... 75

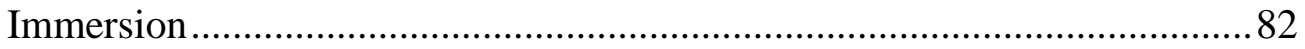

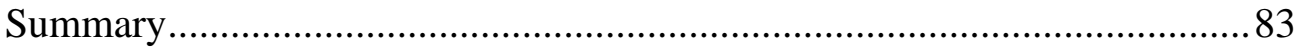

CHAPTER V FINDINGS PART I: ILLUMINATION AND

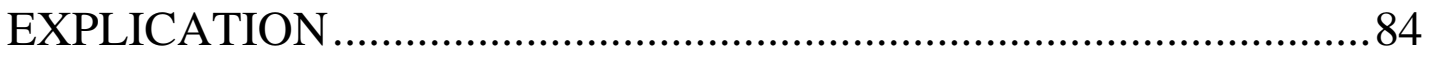

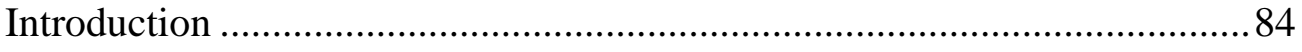

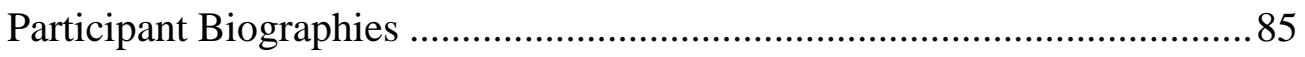

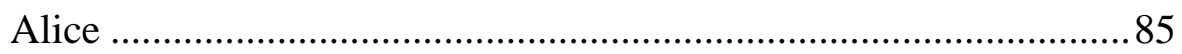

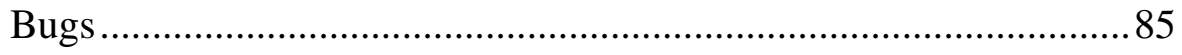

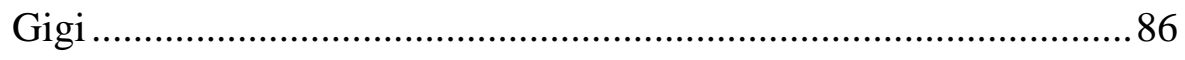

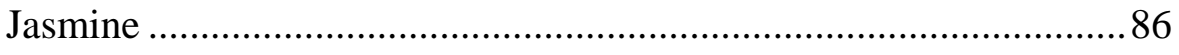

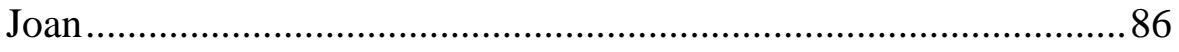

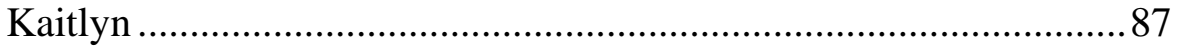

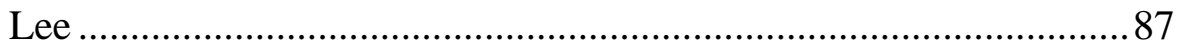

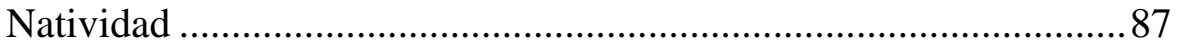

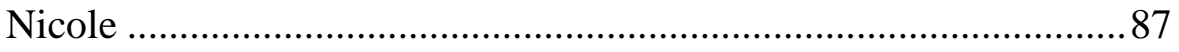

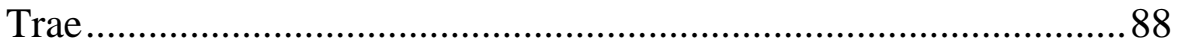

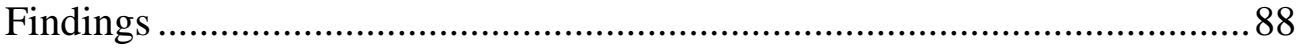

Having Blind Trust ........................................................................ 89

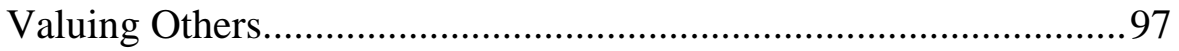

Transforming experiences ...........................................................103 
My Voice

Summary

CHAPTER VI FINDINGS PART II: EXPERIENTIAL LEARNING AND THE DIALECTIC

Introduction

Narrative pedagogy

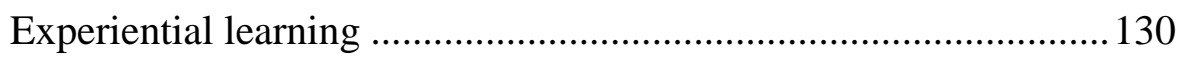

Dialectic Analyses.... 132

Have and Have-Nots 132

Being an Insider and an Outsider 138

A World Shrinking, a World Expanding. 144

Conclusion 153

Summary 156

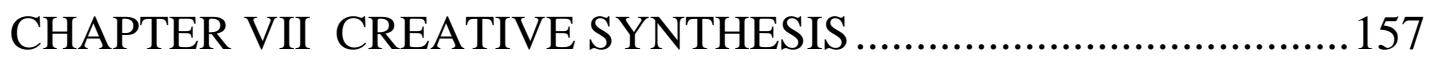

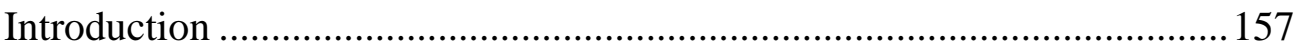

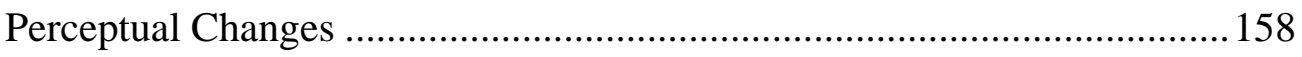

The Influence of Economic Status .......................................................... 161

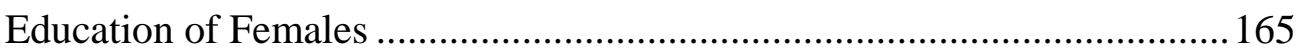

Family: Women’s Roles and Functions ...................................................168

Government Structures and Influence on Health Status............................. 170

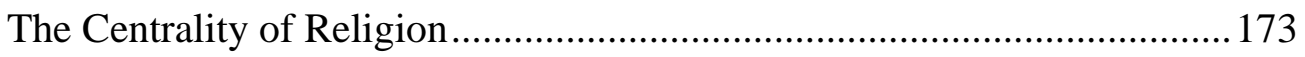

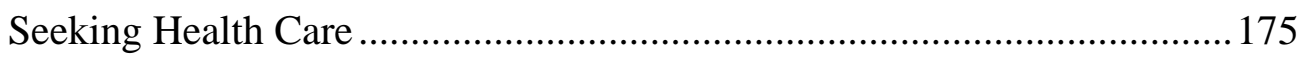

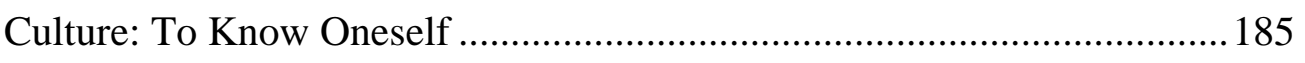

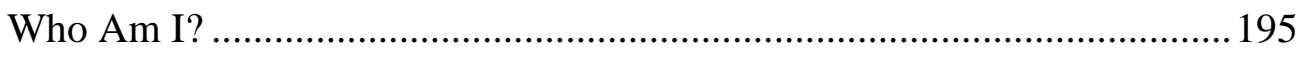

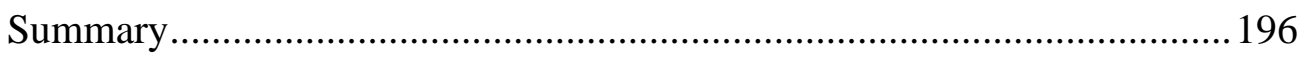

CHAPTER VIII MY EPIPHANY .............................................. 197

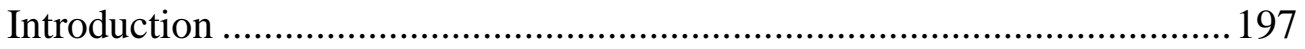

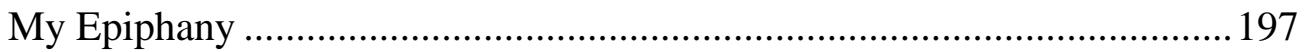

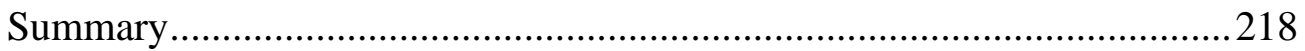

CHAPTER IX REFLECTIONS ON THE EXPERIENCES OF OTHERS: A REVIEW OF THE LITERATURE ..................................219

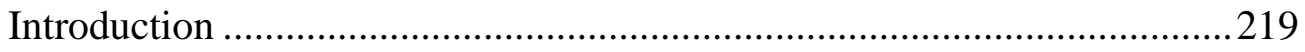

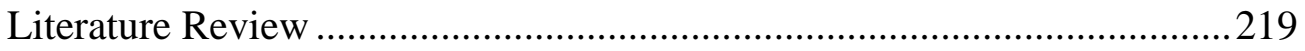


Preparation for immersion ......................................................... 230

Outcomes

Summary

CHAPTER X CONCLUSION: THE TIE THAT BINDS: PULLING IT ALL TOGETHER

Introduction 241

The Evolution of This Project 241

Why This Study Matters 246

Study Limitations 248

Future Studies Needed 250

Summary 252

CODA 254

APPENDIX A INVITATION TO PARTICIPATE ..........................255

APPENDIX B CONSENT FORM-TRANSCRIBER........................258

APPENDIX C CONSENT FORM-PARTICIPANT ........................260

APPENDIX D PREAMBLE UPON TAPING ...............................262

APPENDIX E ETHICS APPROVAL ….........................................264

APPENDIX F EXAMPLE OF TRANSCRIPT PAGES WITH ANALYSIS 266 


\section{LIST OF TABLES}

Page

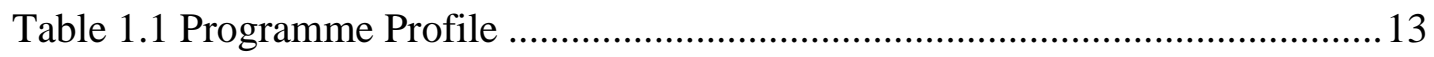

Table 2.1 The Moustakas Model of RTA...............................................................52

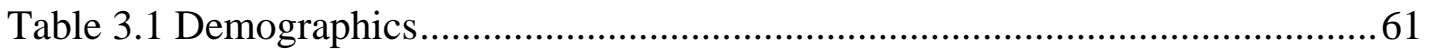

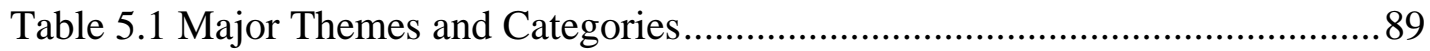

Table 9.1 International Immersion Programmes .................................................. 238 


\section{CHAPTER I}

\section{INITIAL ENGAGEMENT}

“To 'do educational justice' is no simple matter. It means teaching with hope and integrity, moving beyond bureaucratic fixes.....Most of all, it means having the highest respect for our profession, for the work we do, and for the students with whom we work."

(Nieto \& Gordon, 2002, p. 75)

\section{Introduction}

Nursing students for the last twenty five years have faced a rapidly changing practice arena. Indeed the world itself is a setting for change. It is an absolute necessity for today's students to understand who they are and what they bring to the profession and practice of nursing/midwifery. It is essential for each to understand her/his cultural underpinnings, philosophical stance, and what triggers personal responses, be they negative or positive, in order to impart greater understanding and effectiveness as they assume their professional roles with patients. It is also imperative for each to be knowledgeable of the impact, on humanity, of the increasing globalisation of the world.

With this imperative in mind, an international, perinatal, immersion programme for student nurses was developed in 1985 order to promote students’ evolving world view. The international programme for the Department of Nursing at Humboldt State University followed my own transcultural interests in a variety of professional roles in Africa, Great Britain, India, and South America. My volunteer roles included nurse, midwife, resource person, mentor, teacher, facilitator, planned change agent, friend, confidant, and "cultural ambassador" from the developed world. This has dovetailed nicely with what has developed into the international immersion programmes at my university.

In this chapter I will describe my philosophy of nursing education, my nursing background, the programme logistics, theory components, clinical aspects and how they are linked to this research project. This chapter contributes to the thesis by setting the scene, in detail, and affords the reader indepth insight and 
understanding of the immersion programme. My international work is being presented to you my reader, as part of my life's work and doctorate in midwifery at Victoria University of Wellington, New Zealand. I seek to share my reflections on my 30 years of travel both in and out of the United States of America (USA). Travel, especially travel which involves immersion has a powerful impact on people. This impact is even greater on students who are both less worldly and are experientially young. This chapter sets the stage for this project and, in turn, my thesis.

\section{My Teaching Philosophy}

My teaching philosophy includes the belief that learning should be an active and interactive process initiated, stimulated, motivated, and evaluated by both learner and teacher. I also believe that the responsibility of a teacher is to facilitate, promote, and inculcate the never-ending quest for knowledge and its application. As a teacher and facilitator, I endeavour to provide this by providing an environment of freedom, inquiry, and experiential opportunity both in formal and informal settings. As an educator, my devotion to my students, as a mentor, clinical specialist, and theoretician have been my utmost priorities. Overall my philosophy includes a staunch commitment to all of my students to foster their professional and psychosocial growth and development so that they may assume a positive professional as well as social role in society. "It's [all] about students. Why we're here, why we care, what makes us want to continue to do what we're doing-as educators... our very purpose for being is 'about students”” (Accord, 2000, p. 128).

From the inception of my process of professional development I began writing soul searching “stories” about my journeys and my students' because I was “haunted” by my experiences and the emotions they aroused in me. As a teacher I realised that students needed to experience these same emotions in order to gain insight into their (the students) way of being in the over-developed world and insight into the lives of people who are the same in their humanness-no matter where they live. I knew that I had to take students out of their comfort zone to partake in experiential learning, via international immersion, in order to promote psychosocial growth and change. Being in the USA was just too comfortable for most. 
My international experiences became stories, a series of vignettes. “I didn’t have to create the world I wrote about it. I realized that words could tell. [sic] That there was such a thing as an emotional sentence” (Lorde, 1984, p. 85). The stories evolved into 38 vignettes, each a poignant personal reflection of trying to comprehend and make sense of the multiple realities and challenges of the lived lives of childbearing women, and others, in the developing world. Many vignettes involve students, some do not. The vignettes are unpublished and in this thesis I am using 16 vignettes to illustrate salient concepts related to powerful feelings that will remain with me forever. "Stories give shape to experience, experience gives rise to stories. At least this is how it is for those who have had the freedom to tell their own stories, to shape their lives in accord with their experience” (Christ, 1980, p. 5). I will begin with the following vignette which describes my introduction to professional practice in the developing world.

[name of country] Hip/Pin

This is my first time in the developing world. It is 1971. I am still settling in as I have arrived only 36 hours ago. A very old nun expires. The sister in charge asks me, and a young Canadian doctor to report to the room of the late sister. We are to bring a scalpel, surgical hammer and a few other sundry tools. We arrive and are asked to remove her hip pin as it will no longer be of use to sister. I can easily recall this event as if it occurred yesterday. Grisly, lack of orderliness or control, a possible assault on a fellow human being, but remember, MaryAnne, this person has expired and would want someone else to benefit from what she is able to offer. These are the days before gloves and HIV, but the days of HEP B. This is a difficult job, a tedious one, and if I stop to ponder about what I am doing, I could easily be swayed not to do this. It is difficult to get to the area in question and our surgical tools are questionable. The implanted device has been in situ for a few years, is difficult to remove, and it must remain intact. Much struggling and much improvising, but we get it done. An early lesson in practicality, and recycling!

The experience described in the preceding vignette was my introduction to health care in the developing world. It shocks me still. The fact that we would take a hip pin out of a deceased person for possible reuse was beyond my imagination. I felt physiologically queasy and psychosocially questioned the ethics and morality of what I was doing. I was at once horrified but also gratified. Out of this early 
experience, I realised how profound a human interaction could be. I felt compassion and revulsion. I have often reflected on this most vivid memory and ruminated on the issue of developed world student exposure and placement in a similar venue. It was so powerful that I couldn't help but consider the possible impact on students. I wanted them to grow, to see the world, to practice from a global rather than a local viewpoint. I wanted students to have the potential of life changing experiences, so I developed the international immersion programme. The international immersion programmes have been a strategic focal point of my personal and professional life for many years. I developed, devised, initiated, implemented, planned, participated in, and coordinated all the programmes. I'll next address my qualifications to lead the programme.

\section{Qualifications as Programme Director}

For growth to occur, like with plants, one needs both natural ingredients and energy. The natural ingredients for plants are soil, fertilizer, water and sun. The energy comes from the gardener who tills, tends and encourages the plants to grow. The same is true for students, the stage is set, the "plants" are in place, and I provide the raw material. I am the gardener.

My nursing background, working with a broad spectrum of diverse peoples, both in the USA, Great Britain, Slovenia, and many nations of the developing world contributes to my body of bio/psycho/socio/cultural knowledge. This has placed me in the unique position of being able to understand the importance, to students, of knowing who they are as well as who others are in order to offer the best professional nursing care and caring to their patients and families. The qualifications I have as programme director are both educational and experiential. My education includes a Bachelor of Science degree with a major in nursing from the University of Miami, Florida, USA. I received my Master of Science degree in nursing from the Pennyslvania State University, USA, with a major in Transcultural Health and a minor in administration. I am a British educated midwife, receiving my SCM (State Certified Midwife) Certification from Whipps Cross Hospital, London, England. I also hold Certification as a Health Educator, received from Waltham Forest College, 
London, England. I completed my Diploma of Professional Studies in Midwifery at St. Mary’s Hospital, Manchester, England, affiliated with the School of Midwifery, University of Manchester (whilst on sabbatical from Humboldt State University). For the past five years I have been studying and researching for a $\mathrm{PhD}$ in midwifery and continuing to work as a full time professor of nursing/childbearing health.

I have practised as a nurse/midwife in many developing nations as well as working with multi-ethnic and racially diverse populations in the USA and England. From the inception of my career, I have worked with disenfranchised people and the majority of my publications focus on poor childbearing populations in the developing world. Following is a sampling of places and people with whom I have worked.

In New York City I worked as a Public Health Nurse in a predominantly Spanish speaking immigrant Puerto Rican community, which also served a population of recent immigrants from mainland China and Taiwan. Forty percent of my workload was responsibilities in three primary schools serving the local community. I vividly remember one six year old who drew a picture for me of his home, and when asked what the little black dots and grey "bodies" were, he was astounded that I didn't recognise the roaches and mice.

I was the supervisor of a paediatric accident and emergency department serving an inner city New York high density area. The population was composed of an immigrant Spanish speaking community, an urban Black population, a Gypsy community, a poor White community, and a polyglot mix of people from many nations.

Amongst the many professional positions that I have held the following one stands out embedded in my psychosocial memory. I worked at small hospital in rural Florida on a segregated Black unit, by choice, and before racial integration in the United States. The unit was located in the basement of the hospital, and the one Black doctor had to use the dietary kitchen as his way of entry and egress. I was ostracised by the majority culture nurses because I worked on the segregated unit, but that only served to strengthen my resolve and my feelings about the need for education and building of knowledge about racial, religious, and ethnic understanding. 
In England I was a resource for community midwives concerning the relationship of culture to health beliefs and practices. I was also a research consultant on a study about the perception of satisfaction of immigrant Bangladeshi, Indian, and Pakistani women related to the maternity services offered by the local health authority in Manchester. I worked in Malawi, in central Africa, in a mission hospital that provided services to a local community as well as people in the "bush". We had one vehicle and made monthly visits to outlying communities during the dry season. Pregnant syphilitic mothers, infants with kwashiorkor, marasmus, rubeola, helminthiasis, and malaria were common. Part of my role was to work in the antenatal clinic where health maintenance behaviours, and disease prevention, through teaching/learning, were a priority. In Peru and Mexico, I worked with indigenous peoples. In the Dominican Republic I worked with local people as well as Haitians, as the location was on the border of the two countries. I volunteered at a Mother Teresa Foundling Home in New Delhi, India focusing on abandoned babies. I taught at a nursing school in Suzhou, China and was also a consultant to the faculty. I have also worked with students in the Dominican Republic, Honduras, Mexico, Nepal, the Philippines, Russia, and Slovenia. My roles and functions are diverse, as are the settings. I serve as an educator, resource person, practitioner, facilitator, and liaison person. I have worked with health promoters (community lay people who interface between the health care system and individuals and families) designing and implementing protocols in the Dominican Republic, Honduras, Malawi, and Peru.

Having worked in a wide array of clinical and academic settings in the USA and abroad I feel comfortable in diverse surroundings and locations. I would describe my attributes as flexibility, adaptability, creativity, self awareness, and therapeutic communication. I am well qualified to both participate in and conduct the international immersion programmes. 


\section{Value of Immersion Programmes}

\section{To the students:}

The appeal, importance and significance to the students included living and practising their profession in an environment completely different than they had ever known. There was the opportunity for psychosocial growth and the chance to meet and work with their professional counterparts. There was the prospect to socialise with people whom in the regular course of their lives would probably have never taken place. The opportunity existed for students to immerse themselves into cultures new to them and learn about how other people live their lives. Students were able to practise in health care settings very different from what they had ever experienced and were able to be creative, innovative, and flexible.

This programme also promoted cross cultural exchanges of both professional and personal values. For the American (USA) nursing students it opened the borders beyond their university and country and gave each a perspective of life that was vastly different than that to which they were accustomed. This was a growth experience for students as they realised that there are innumerable elements involved in problems, a variety of ways to problem solve, and a great variation in lifestyles. They learned how to work within the structural framework of vastly different milieus by actually being participants. Also, the students were no longer members of the majority culture, in both their professional and personal lives. Each had to learn to adapt and cope with many new ways: language, the roles and functions of the nurses and midwives, the culture of the health care system as well as the effects of the sociological institutions of the particular society on the individual, family, and community. They adapted to food, water, code of dress, and the social conduct related to personal space, interactions, and the differing roles of health practitioners. These interactions affected the student participant, the citizens of the country and the health care providers. The professional lives of the students and the activities that were undertaken were significant for they modelled the world of the target population and utilised accessible local resources. The resources (props, media) were easily replicated in many of the communities, and could be incorporated into the lifestyle of the patients. At the conclusion of the programme students stated that 
their understanding of lifeways were vastly enhanced. They also felt that productive, relevant and appropriate problem solving could be accomplished in different ways. The assumed benefits of the programmes will be explored further in this thesis.

\section{To the university:}

The immersion programmes were predicated on experiential education that promoted a unique dynamic partnership and exchange among all participants. The concepts of critical thinking, problem solving, value systems, and the creation of a supportive environment were all key elements. This is congruent with Humboldt State University's mission to serve humankind; its commitment to multiculturalism in education; its commitment to cognitive and cultural development of students; and its commitment to lifelong learning. The Humboldt State University, Study Abroad Program (1985) preamble states: “All study abroad programs begin with a concept which ties domestic educational objectives to perceived opportunities for enrichment, specialization, and/or altered intellectual perspective available in a foreign learning environment” (p. 3).

\section{To the discipline of nursing:}

Students were profoundly affected by conditions in the health care system as well as the personal lives of those they worked and lived with, yet they were able to see similarities in differences. Students were able to build cultural health care bridges between two very different health care delivery systems through working with local health care providers.

The immersion programme has always been important because the global perspective of the world continues to shrink and humankind becomes more intimate in its interactions. Students were thus on the cutting edge of today's and tomorrow's world due to their total immersion into the institutions of a society that was geographically external to the one that they lived in, and completely different from what they had ever experienced.

Students practised their profession with nurses, midwives, doctors, and ancillary personnel whose roles and functions vastly contrasted with their own education, practices, and philosophies, and lived and participated in the social milieu 
of the citizens of the communities in which they worked. This meant new sights, smells, sounds, tastes, friendships, vistas, and living conditions, all of which required the staples of flexibility, adaptability and therapeutic use of self.

At the conclusion of the programme students' understanding of life was vastly enhanced; participants realised that productive, relevant, and appropriate problem solving can be accomplished in many ways and this opened up the pathway to understanding diversity in their own home communities, and nation. Students felt comfortable working with clients whose modus operandi was different than their own. They also learned about their own culture, who they were, and what they brought to client interactions. "My student” refers to a student and faculty relationship necessary to maintain a supervisory and educative role in place. This term will be used in the thesis to denote this close teacher student relationship. At times the pronoun "we" will be used to denote "us" as distinct from others in the setting and to emphasise other relationships.

\section{To the hospitals and community health facilities:}

We participated in the direct provision of health care to perinatal women, infants, young children, and others. We engaged in health education projects, incidental and formal teaching, direct patient care in both hospitals and community centres, worked directly with local nurses, doctors, midwives, ancillary personnel as well as student nurses and midwives. Most important of all was the camaraderie and understanding that existed among my students and the health care personnel at the immersion sites.

\section{The Programme}

The international perinatal immersion programme was designed to meet the stringent requirements of Humboldt State University, and the California State University system. Each summer's programme (Study Abroad Program, 1985) was completely reviewed and approved by the chancellor's office of the California State University, as the venue for each placement was different. The following sections were included in each application: The Curriculum; Personnel Matters; Evaluations; 
Logistics, Services, and Finance; Housing and Food Services; Health and Safety; Program Budgeting and Financial Aspects; and Student Recruitment and Selection.

The Study Abroad Program (1985) was a seven unit course comprised of one lecture unit and six clinical units. It was a total bio/psycho/socio/cultural immersion into the health care system and culture of another country. A few examples are: The Dominican Republic, Nepal and the Philippines. The patients with whom we worked were individuals, family units, and entire communities, ranging from rural isolated small villages to large metropolitan centres. Students and faculty were partnered with local care providers including nurses, midwives, doctors, health educators and ancillary members of the health care team. The overwhelming majority of clients we served were impoverished. All were culturally diverse and many had little or no formal education. Many had severely limited access to health promotion, maintenance, or restorative services. Specific countries, cities, and people will not be identified to protect the identities of the countries involved, the health care personnel and the students. I will indicate where incidents have taken place in the following manner, by placing a [line] instead of identifying the venue.

The curriculum was composed of a theory segment that took place during the spring semester, followed by the clinical placement immediately following in the summer. The theoretical concepts of planned health change strategies; lifestyle and its cumulative effects on individuals and communities; the constructs of society that directly affect societal roles; empowerment of clients through individual and community participation; utilisation of local resources: people and materials; were some of the topics that students researched, presented and discussed in the theory class.

I have always utilised a sociological framework as theory base as societies share the commonalities of the institutions of education, economics, family, government and religion. These institutions are found in many forms and vary from the rudimentary to the extremely complex. The deconstruction of a society's multiple institutions enabled students to analyse, diagram and understand the components of a social system by being able to separate the functions of each institution and its cumulative effects on the life of individuals, families and 
communities. Students chose which institution they wanted to research and presented a formal lecture followed by a discussion, which they chaired.

Transcultural Nursing and Cultural Safety theories were also topics to be researched and presented. Basic language preparation was also addressed and when possible native speakers conducted these sessions. An example of a student presentation included: The education system in the country to be visited in order to provide perspective and also a comparison and contrast to the same institution in the USA. The academic requirements of the theory class included one formal paper, one presentation and discussion of a researched topic and intensive class participation. The class met for two hours every other week for an entire semester.

Requirements for the clinical (summer) placement included six to eight weeks of professional practice, daily “journaling”, related to professional role, socialization, and reflection of feelings. Four weeks after returning to the USA a formal annotated subjective paper was due. This culminating paper enabled students to integrate the entire experience utilising the journal, reflective practice and references from at least five relevant professional journals/books. This was a synthesis of the entire programme and its individual meaning to each participant at the time of the writing.

The participants ranged in age from 21 through 48 years of age. Several were married and a few had children. Usually 40 students expressed interest in participating. Random selection was used due to the high numbers of applicants which greatly outnumbered the placements available; limited accommodations reduced the number of students who could participate in the programmes. One of the students once described me as a person who "wore many hats", as I had an enormous amount of responsibility for the total welfare of each student and fulfilled many roles and functions.

The structure of the programme at the clinical site was as follows. Students were assigned to units in pairs. I always teamed together the upper class student, who had the most experience, with the lower class student in order to help promote leadership in the former and confidence in the latter. Each pair was assigned to a hospital, clinic, or community rotation for approximately 32-40 hours weekly with rotation to new clinical sites occurring approximately every two weeks. There was 
little or no technology and sometimes the spoken language was not always a common one. Each programme was unique in its clinical settings and assignments directly related to the existing facilities.

During the international clinical component we convened daily or every other day to reflect upon our activities, compare and contrast experiences, and develop aims, goals, interventions, and modalities of evaluation for our projects. This was an essential part of the programme as students, individually and collectively, explored their feelings and reactions to everyday professional and personal occurrences.

In some instances we were supported by translators. In Russia we had four interpreters with us on the hospital units and in the community assisting with tasks such as police clearances, and getting our money changed into rubles. In each instance this seemingly common activity was a major event for the students and the Russian bank, as foreigners rarely travelled to [name of city]. Students and interpreters developed deep connections with each other as they worked closely together everyday and also shared many social experiences out of the work environment.

On many occasions, on the outbound or returning segment of the clinical component there was a unique intermediate stop at a School of Nursing/Midwifery. The aim of this was for students to liaise, and compare and contrast their own programme of nursing, and their general university education with that of their peers in a foreign university. The students also socialised with their peers and learned about the psychosocial constructs of their lives by participating in activities together. Three sites were: Rangsit University Department of Nursing and Midwifery in Bangkok, Thailand, on the way to Nepal; the University of Munich, Department of Midwifery on the way to Slovenia; and the Kandang Kerbau Hospital in Singapore after leaving the Philippines. These were valuable experiences for the students as they could identify with their peers and share many similar experiences of student life, especially, as students of nursing and midwifery. There was a feeling of cohesiveness, closeness and understanding. The visits lasted approximately one week. 
The programme was piloted in 1985. Since that time a further six groups of students have participated in the immersion programmes. A list of locations, years and numbers of participants in each programme follows in Table 1.1.

The locations of the programmes were informally negotiated through my own international contacts, and on two occasions through the Catholic Medical Mission Board. Student selection was unique as it was not based on scholarship. The only qualifying factor for the Humboldt State University programme was that the student had to have completed the first year of the nursing programme. My personal feelings have always been that a high grade point average would not be the qualifying factor for participation and that all nursing students should have an equal opportunity.

Each Study Abroad Program (1985) was offered as an elective and a voluntary overload for me. I had (have) many feelings about this issue and have constantly had to "work through" the administrative challenges in order to offer the programme on a tuition free basis. I did not want students burdened by both the

Table 1.1

Programme Profile

\begin{tabular}{lcc}
\hline Venue & Year & Number of Participants \\
\hline Russia & 2000 & 11 \\
Nepal & 1999 & 9 \\
Slovenia & 1997 & 9 \\
The Philippines & 1996 & 10 \\
The Dominican Republic & 1994 & 3 \\
Mexico & 1991 & 8 \\
Honduras (Pilot Programme) & 1985 & 1 \\
\hline Total Number of Participants & & 51 \\
\hline
\end{tabular}


costs of travel and room and board as well as tuition. I also never sought financial remuneration as I felt (feel) very strongly that being able to offer an expertise to others must not always involve payment.

There were several reasons that I chose to take students to the developing world. I hoped that participants would learn that poverty has relative meanings. For example, the typical American university student may consider herself/himself poor which can then be addressed related to the abject poverty they were exposed to. Poverty can render individuals completely "faceless" in their society which, in turn, can make the individual powerless in the society in which he/she lives. Living in dire poverty denies individuals access to formal education, which also makes access to health care extraordinarily difficult or even non existent. Poverty may also be associated with the individual, or group, who does not belong to the majority culture which can greatly impact lives. Another reason for differing venues is that I, as the programme initiator, director, and accompanying faculty member felt that learning would be maximised in different settings rather than returning to the same community over and over again.

The airfare consumed the largest outlay of money: on a few trips it was as much as $\$ 1200.00$ United States dollars. Despite these high costs students chose to participate. Each participant was responsible for his/her own financial arrangements that consisted of grants, loans, or even credit card cash advances. The cost of each programme typically involved: airfare, room and board, passports, visas, health insurance (including return in a body bag, should the situation arise), immunisations, plus a few miscellaneous expenses. The average cost was about $\$ 2000.00^{1}$

Housing was pre-planned through the sponsoring agency. We were accommodated in the homes of the local populace, sometimes with priests and nuns. We paid from \$25.00-\$35.00 (United States currency) weekly for room and board. We ate with "our families" bathed as they did, in rivers, or with a bucket of rainwater, boiled and filtered our drinking water, and socialised with their friends and relatives, and we became part of their social network. On one trip we lived in an orphanage and were surrounded by 55 children of all ages. During our non-working

\footnotetext{
${ }^{1}$ US dollars used due to its recent devaluation which would not reflect the actual costs if NZ dollars were used.
} 
hours, we had many wonderful hours playing with the children. Probably the most common overwhelming event for us, at some sites, was the incessant and unrelenting heat from which there was no escape.

The beginning of a typical day is portrayed, as follows.

[title not included as it names the city and the country]

Out of bed at 0600 having been awakened long before by the unfamiliar sounds of the city, the heat and the swarms of mosquitoes. The rat in our room has been active during the night and we could hear it scurrying about; this gave rise to fear but physical exhaustion permitted us to sleep with only brief waking moments accompanied by the noises of the rat.

Morning activities of daily living are completed, including ensuring a litre of potable portable water for the day. We prepare for our clinical placement at one of the city's maternity units, or a placement, in a rural nearby village, with the nursing students of College [our primary hosts]. Our breakfast consists of rice, shredded beef in hot sauce, bread, oranges, and tea. At 0730 we board the School of Nursing bus. The American [USA] students are paired with the local nursing students. The local college nursing students look fresh, clean and crisp in their white blouses, blue trousers, and dark shoes, while we are dripping with perspiration, our uniforms soaked, and our hair looking as if we had just stepped out of a shower. We are clutching our water bottles, our most precious and prized possessions.

No matter the amount of theoretical and practical preparation for this journey, the physiological processes still rebel at the intense heat and humidity to which we are not acclimatised. As today is our first day we do not have the requisite handkerchief, or scarf, to cover our noses and mouths as we proceed through the city to our destinations. The bus has both open and broken windows. The dust and exhaust fumes from every type of vehicle imaginable, the blanket of smog and the cacophony of claxons, people, and animals are outstanding to our ears.

The differences in cultures are dramatic. The majority of people raised in the developed world would have a difficult time adjusting to the daily rigours that people who live in the developing world need to survive. The statement "We take our own standards of life so much for granted, that we simply have no conception that they are not universal” (Williams, Baumslag, \& Jelliffe, 1994, p. 29), rings especially true as students negotiated their way through the city. 
As I reflect upon this vignette, which describes a typical developing world major metropolitan area, what comes to mind is the stark contrast of the lived lives of the students from the USA versus their "here and now” spontaneous living. The amazing "jump” from developed to developing world is as a chasm thousands of kilometres in length with no intermediate stops permitted. I think about the romanticism of the students who expected some quaintness, crowdedness, and grime versus the actuality of the squalor and detritus as each experienced a new reality. I ponder the potentially profound effects that this immersion experience may precipitate. Will each student begin to view life through an altered lens? I consider the effects of the assault upon the senses: sights, tastes, smells, sounds, feelings, never ending differences. The effects could be disconcerting and jarring. I intuitively know why I am doing this for it is absolutely necessary for students who grow up in a “cloistered” environment with multiple “creature comforts” and lack of exposure to the realities of the lives of others; true-life exposure.

The summers' clinical immersion projects were always decided upon with the input of community members. Students had the necessary tools from the theory segment of the programme, from their prior nursing courses, and from their knowledge base in the pure and social sciences. They used their critical thinking skills for problem solving within the community(ies). We met with the families, children, shopkeepers, religious leaders, other key people, and conducted open fora in order to ascertain the community's perceptions of their health care needs. We then worked within the community to prioritise their health care needs, and throughout our stay continually liaised with its members.

We were consistently well received by staff and worked with them as part of the health care team. Students would receive invitations to visit the homes of the people they worked with and relationships would develop. In many of the nurses had never met a foreigner, and told us that they never, ever, envisioned working with nurses from another culture and how much they were enjoying the experience. Students were invited to eat lunch with the staff, and treated as if they were employed by the institution(s).

When students were not at work and went out to explore, I always insisted that they go in pairs. This was a policy that I have always rigidly enforced in order 
to maintain safety and security. Students were also required to be dressed in a conservative manner and also appropriate to the setting of the country.

At the conclusion of the clinical component we would spend a few days in rest and recreation and preparing ourselves for the return to the over-developed world. Students used this time to get ready for the physiological and psychosocial transition necessary to return to their former way of living.

In concluding this chapter about the immersion programmes it is important that I address the evaluation tool that I used at the conclusion of each programme. It truly failed to capture the essence and value of the experience for the students. The tool was a typically standard university evaluation form to look at data for trends. Upon reflection of the questions (such as: lecture content relevant and applicable? learning activities relevant to course content? the instructor's general knowledge of the subject matter was? and organisation and clarity of lectures) there was a failure to capture the real meaning for programme participants.

\section{Study Abroad Programmes}

The impact of the study abroad programme on students subsequently became an area of focus and personal exploration for me. While I used to believe that I fully understood the students' experiences, I began to question if my own background and history may have caused me to misrepresent or misinterpret the meaning of the students' experiences abroad. I began to recognise that I was fairly "set” in my own patterns of thought. I began to reconsider whether it was really possible to enter “another world” and “walk a mile in someone else’s moccasins”. It became obvious

that I could only meet people "where they were”, from a place of "where I was", a foundational element of the concept of Cultural Safety (Ramsden, 1990, 2002). I felt that I could question this gap in perception through my students' experiences. Therefore I have undertaken this research project.

This thesis explores the impact of my life's work practising the professions of midwifery/nursing and immersing myself in many cultures around the world. It critically examines the purpose of my going abroad and the inclusion of nursing students in providing health care in hospitals and community settings in many 
countries. I have worked outside the USA in an effort to further engage with people of other cultures, to utilise my professional skills, and learn from others. This thesis investigates the experiences and perceptions of students and also my motivations for undertaking international immersion projects.

I realised that I am passionate about “opening the eyes of my students” to their own world and the developing world. Through reflecting I wondered: would students be able to truly see diversity, difference, suffering, the disrespect and disregard for others that people of all cultures are capable of developing-for those outside their world view? Could students be both insiders and outsiders at the same time? Would students be in an uncomfortable space, the space where I live every day-knowing what I know and have experienced?

The thesis is about me, and my search through intense reflection, student interviews, my slide collection, vignettes, and literature review, to identify my subjective experiences and arrive at a deeper understanding of self. The theoretical framework utilised is Moustakas's (1990) heuristic process and Reflective Topical Autobiography as described by Johnstone (1999) because these methods focus on the lived lives of individuals.

The following section describes the layout of the thesis and provides an overview of the content of each chapter. Chapter II discusses the theoretical underpinnings of the research starting with Reflective Topical Autobiography (RTA). The chapter then addresses, biography, autobiography and interpretive materials and their relevance to the research. This chapter reveals the somewhat unusual nature of this kind of research endeavour and asks the reader to set aside more formal notions of an easily defined and time limited piece of research that investigates a single question. What will be explored and presented in this thesis is a life-work of practice and reflection on practice. This research spans 30 years, involves many individuals and many countries and will continue to evolve even after the thesis is presented.

Chapter III provides a detailed examination of the writers and researchers who have provided the insights into methodology and inspired the methods used in this study. In keeping with the heuristic process at the heart of Moustakas’ (1990) model of inquiry and Reflective Topical Autobiography as utilised by Johnstone 
(1999), the specific steps I followed are provided to guide the reader throughout the thesis. These steps begin with the study design and include a review of RTA, my perspective of this project, and the use of vignettes as a tool of description and reflection. The chapter continues with the rationale for the inclusion of several biographical accounts of the immersion programme provided by a selection of past participants. In this chapter I argue that each source of material; reflections on my life story and perspectives provided by the student stories contribute to new ways that teachers can utilise “...life experiences (epiphanies) that [may] radically alter and shape the meanings persons give to themselves and their life projects" (Coffey \& Atkinson, 1996, p. 145).

An autobiographical account of my personal and professional life journey and my family of origin is provided in Chapter IV. In writing this chapter I discovered and reflected on the experiences that shaped the person I am today and the forces that drive me to invite students to share some of these experiences. This chapter is an integral component of the thesis because it exposes emotion and passion for positively changing the lives of all I meet and reveals the role this thesis will play in that process.

Chapter V reveals the stories of 10 students who participated in the immersion programme over a span of 15 years and seven countries. The first level of analysis of their transcripts is provided. This revealed profound reflective processes. There were many common themes expressed by the students, which then became three major categories, and added to my deep reflection and ultimately factored into my epiphany. The results were garnered from a wealth of material so graciously given. In this chapter I talk about who I am as this fits well with the students' "exposure".

In continuing to reflect on the students' stories and my own story it became apparent to me that there was a deeper level of analysis that needed to be undertaken. Chapter VI therefore reveals a second level of analysis that explores the dialectic. The educational theories of Diekelmann, narrative pedagogy, (1993, 1995, 2001) and Kolb (1984) and Vygotsky’s (1926/ trans. 1977) experiential learning are introduced as both address innovative learning techniques. Through the use of narrative pedagogy, the sharing of daily lived experiences, a constant thread woven 
throughout the immersion programmes, interpretive discourse was ever present and engendered students to think “outside the box”. Students, through their experiential learning, went through a transformative affective process that opened "new worlds" and new ways of thinking and understanding as their current "constraints" blossomed beyond their known boundaries Chapter VII discusses my personal thesis journey and also the students' realisation of the influence of a country's sociological institutions upon each and every citizen. Students were profoundly affected by working with men and women who had no formal education yet were able to affect changes in the lives of others. This chapter addresses students' constant awareness of "differentness yet sameness" of their home country and the immersion venue. I answer the thesis questions in this chapter and how I have been profoundly affected by my students. This project is about culture and I ruminate about knowing oneself, the most important factor. I talk about who I am and how I came to be that person, which is imperative using RTA (1990) and the Johnstone (1999) models.

My epiphany is uncovered in Chapter VIII, “eureka”, simple yet many years in the making and unfolding. There is indepth discussion of who I am and why I do what I do. Through this writing, and reflection, I have come to understand my intuitiveness and how I have always been guided by my feelings. This information revealed makes me understand why I am driven both personally and professionally to my commitment to poor childbearing women in the developing world and to my commitment to my students and the immersion programme. Using RTA (1990) and Johnstone (1999) I constantly reflected, “talked to myself”, and others, and then scoured the literature.

In Chapter IX I reviewed the literature of international programmes for baccalaureate nursing students for the years 1984-2005. This is also in keeping to the traditions of Moustakas (1990) and Johnstone (1999), and is one of last steps of the process. The majority of the programmes reviewed were anecdotal and few were formal studies. Most of the programmes addressed personal and professional growth as a major focus. I believe that this project delves into depths heretofore not fully explored by others. I also use the metaphor, “take your children to church, don't 
send them”, as an appropriate commentary, for the "journeys” were more meaningful to students when accompanied by faculty.

The conclusion of this project is the focus of Chapter $\mathrm{X}$ and pulls all the threads together. My modus operandi, in relationship to students and immersion programmes, through shared humanness, understanding, titillating all the senses, and cognition, is presented. I am acutely cognisant of the shortcoming of this project through the limited number of participants in such programmes, due to the constraints at the site, and the inability to "support” large numbers of students. I am also painfully aware that there is much to be done in the international arena if this world is to survive.

\section{Summary}

This chapter has begun to describe my personal professional experiences of working in many different cultures which led me to develop an international immersion programme. Chapter I has also described in detail the immersion programme itself and its purported effects on a variety of disciplines and institutions. The ordering of the thesis is also summarised in this chapter. The following chapter discusses the theoretical underpinnings of the design of this study, Reflective Topical Autobiography (RTA). RTA is a qualitative research method that utilises an in depth individual journey with an end focus of a personal epiphany. Similar research tools, such as biography, will also be discussed. 


\section{CHAPTER II}

\section{IMMERSION}

"Two roads diverged in a wood, and I-I took the one less traveled by, and that has made all the difference"

Robert Frost (1921, p. 9).

\section{Introduction}

This chapter discusses the theoretical underpinnings of this study beginning with Reflective Topical Autobiography (RTA) and its evolution as a research tool. RTA is a form of qualitative research which seeks to identify in depth social and professional activities of human beings from a variety of data sources. This study specifically focuses on my journey to an epiphany and my increasing understanding of the experiences of nursing students engaged in international immersion programmes. Autobiography, biography and interpretive materials, as they relate to this project, are also discussed in this chapter. Autobiography in this thesis represents both self perception and that of student informants. This broader use of the term auto/biography is an important and unique derivation of this qualitative research method. Similarly, biography refers to published or otherwise accessible writings often bound by origin or focus. Because of the heuristic basis of RTA, that is a reliance on words and meaning, autobiography as traditionally understood is too limiting to capture the depth and breadth of the immersion programme. By expanding autobiography to include both personal reflection and a reflective process using key informants and other data sources, a richness of meaning far exceeds simple descriptive terms. This novel use of auto/biography is an important and legitimate extension of RTA, completely in alignment with both theoretical and pragmatic applications of the method.

For the purposes of this research endeavour, the practice of nursing and the term "nursing” includes the care of perinatal clients (antenatal, labour, delivery, and post natal), as well as clients in all generic nursing specialities. In the USA, nursing 
and midwifery blend concepts whereas in Australia, Germany, New Zealand, the United Kingdom, and many other countries, these disciplines are discrete. In the USA the midwife is a nurse practitioner whose speciality is midwifery. For my project, any term related to my nursing practice infers that I am practising as a midwife.

The discipline of midwifery, like other disciplines, extends its art and science via carefully constructed research. Midwifery relies both on quantitative and qualitative studies to answer its most pressing questions. Quantitative research remains valuable in that it precisely measures and describes phenomena, and qualitative research in midwifery adds the indepth, contextual perspective that captures the human experience. This chapter addresses the qualitative research process of reflective topical autobiography (RTA) and the use and application of the autobiographical method in answering my research questions. Also described is the Humboldt State University Department of Nursing international immersion programme for baccalaureate nursing students as an important source of information in understanding my "turning point” experience. The core questions underpinning the research of my professional practice and teaching were:

What impels me to return again and again to work with poor childbearing women in the developing world?

How did international immersion programmes impact the personal and professional lives of student participants?

In addition I explored the interplay between my life experience and the students' experiences. I wondered,

Can the student experiences help me to understand myself better?

These questions were always on my mind and in my heart. The following vignette portrays some of my reflections, perceptions, and views. 
I have often thought about my continuing motivation to work in the developing world and the effects that it has upon people whose lives have been affected by immersing themselves into the health care system, culture, and social systems of their fellow sojourners on this earth. It is psychologically difficult to sit and ponder the lives of the many people with whom I have worked and lived as the pain is still so real today. It is often said that a picture is worth a thousand words. Poignancy, despair, hope, and other human emotions may be readily transmitted in pictures, ascertained by facial expressions and body postures; yet how does one articulate the reality of smell, heat, filth, abject poverty and the nuances of people's everyday lived lives? How best to describe an accurate "picture" of the woman whose paid employment is hauling twenty kilos on her back for eight to ten hours daily with two young children accompanying her on her rounds, who go "home" to a cardboard dwelling that offers no protection from the rains nor the ceaseless noise from the main city entry highway that borders the "empty" lot that she shares with other squatter families. The toilet is wherever one can squat and water is hauled from a communal pump about 200 meters from her domicile. Cooking takes place, once a day, on a wood fire, the staple for all is rice. This woman is lucky she has paid employment, there are others living here, whom with their children, stand amidst the traffic begging.

Herein, I believe lays the inexorable lure that for me is inescapable; the dichotomy, the separation, the very distinct divisions between two worlds, the have and the have nots, the filthy and the filthy rich. How can it be, the world of plenty existing alongside a world of hopelessness, grief, and the monstrous loss of neonates, infants, young children and childbearing women? The future of a nation belongs to its children and yet, "we” allow the perpetuation of constant death of the young. I also look upon “other” as a fellow human being, another member of the human family, who is also striving toward common human goals that we all share.

This important research topic lends itself to qualitative study; in particular RTA (Reflective Topical Autobiography). This marriage of topic and method works because of the rigorous demand for introspection and systematic study of normal “real life” phenomena. New understandings are gleaned from what looks, on the surface, to be ordinary experiences. As a result these first three chapters may seem somewhat redundant. I am simply weaving the several layers of my research methods together. I am illuminating different facets akin to the layers of life and 
there are some mirror images that possibly represent how the mind both holds and then reveals, during reflection and contemplation, memories of one's life.

\section{Theoretical Underpinnings}

Qualitative research has been described as that which is particularly helpful in facilitating and creating our understanding of social settings and the dynamic flow of lived lives (Gillis \& Jackson, 2002, Streubert \& Carpenter, 1999). This essence of qualitative research is especially useful because cultural observations are complex and multilayered and "capture multiple versions of multiple realities” (Coffey \& Atkinson, 1996, p.163). The use of textual data and description lend themselves to the richness of qualitative methods (Capera, 1983; Creswell, 1998; Parse, 2001; Polit, Beck \& Hungler, 2001; Ray, 1985). Denzin (1989a) stated "The focus of the research is on those life experiences (epiphanies) that radically alter and shape the meanings persons give to themselves and their life projects” (p. 510). Leininger (1985b) and Morse (1997) discussed the importance of qualitative research and its contributions to nursing knowledge because world view(s), value systems, mores, ideas, convictions, thoughts and processes can be uniquely described, captured and effectively portrayed in a variety of forms.

In the 1980’s, M. Carter, (1985), Ray (1985) and Tinkle and Beaton (1983) observed the movement of nursing away from positivism as the most important method of scientific inquiry. These authors theorised that causality and direction were in opposition to the overarching aim of professional nursing which is to understand and gain knowledge about "the multiple contexts in which [life] phenomenon occurs” (Hinds, Chaves \& Cypess, 1992, p. 32). This thesis aligns itself with multiple contexts consistent with a richly qualitative method.

Today when one looks at the profession of nursing one sees that: "Nursing is an academic and practice discipline” (Wood, P., 2002b, p. 40). This means that nurses and midwives must be prepared to initiate, complete, publish and circulate relevant research. The profession of midwifery is predicated on discipline expertise, evidence based practice, a lifelong commitment to learning, flexibility, creativity, adaptability, critical thinking skills, caring, respect, decency, and trust. In order to 
continually expand one’s nursing and midwifery knowledge, and commitment to humanity, research is critical. This study has been completed in an effort to contribute to ongoing nursing/midwifery-based development and acquisition of knowledge. In order to provide a framework for my exploration of the research questions I examined a number of different theoretical approaches until I discovered, Reflective Topical Autobiography (RTA) as described by Johnstone (1999) and Moustakas (1990). This chapter sets out the details of RTA and provides a justification of why an autobiographical approach is the most appropriate for my inquiry.

\section{Reflective Topical Autobiography (RTA)}

RTA is a form of autobiography focusing on the lived experience of an individual in order to identify human commonalities. The overall purpose of RTA is to use one's own richly subjective experience as a method of studying and understanding some of life's most emotional, psychological, sociological, interpersonal and intra-personal realities. I am immersing myself in how to explore the questions that my life and teaching have raised for me. RTA is a method that “...advances...the understanding...of human experience” (Johnstone, 1999, p. 25) through probing the innermost recesses' of one’s personal life experience. “To be a self is to be... the subject of one's own experiencing” (Jourard, 1967, p. 1). This means one person, on reflection, can retrieve the wisdom from within. The process includes dissection, rebuilding and reconstructing a pivotal phenomenon of one's life in order to come to a new deeper reality. Behar (1996) addressed "inscribing of the self” in utilising evocative, colourful, prose. Metaphorically speaking, RTA is an acappella performance, a solo voice, raised in an empty theatre that furthers human understanding of what an actual individual has experienced. Nieswiadomy (2002) stated “...the individual's perspective is very important” (p.150) in gaining insight and understanding of the human condition.

RTA is an offshoot of several similar autobiographical processes that attempt to examine, scrutinise and document one's own life through biographical methodologies. Generic to the biographical model is "the studied use and collection 
of life documents that describe turning-point moments in an individual's life" (Denzin, 1989a, p. 69). Moustakas (1990), Plummer (1983), Rowan (1983), and others (Barnes, 1981; Brettell, 1997; Chalasinski, 1981; Frick, 1990; Giele \& Elder, 1998; Johnstone, 1999; Jourard, 1971; Kadar, 1992a, 1992b; O'Rand, 1998; and Watson \& Watson-Franke, 1985) agree that rich wisdom comes through autobiographical and biographical review. Because RTA is based in personal reflection and thought, a wide variety of classic and current reference works have become embedded in the process. This deviation in the current practice of using only contemporary writings is being abandoned to allow more indepth contemplative activities. Moustakas (1981) emphasises “...intuition, spontaneity, and selfexploration are seen as components of unified experience in which both discovery and creation are reflections of creative research into human ventures, human processes, and human experiences” (p. 216). The following vignette describes my overwhelming feelings.

Tuna/Indigenous Young Boys

A student, my only daughter at this time [10 yrs of age] and I are exploring the countryside and the indigenous small village communities of a nation in the Americas. We have just finished working in a neighbouring country and are on rest and recreation in order to regroup before returning to the developed world. It is the time to seriously consider "our" and "their" lived experiences.

We arrive on market day in one town and it is bustling with locals and foreigners eager to purchase indigenous made goods. We wander around the town's environs and the market; easy to make friends as my daughter, an indigenous North American, looks like the local populace yet does not speak their language nor Spanish and so we spark many a conversation and are made to feel at home. When we return to our rented car we have a perfectly flat tire and know that this will take hours to remedy. I bring out our emergency rations of tuna in a tin, and an opener [I always carry staples because very often there is no place to obtain food]. We have crackers, and carry potable water. When we have finished eating I notice two very emaciated and hungry cats, and give the remnants of the tin to them. All of a sudden two boys materialise [about five years old] and ask me why I gave that food to the cats and not them. What I felt was overwhelming and absolute shame. 
I still feel the shame. I have been constantly barraged by scenes similar to this, commonplace to my life in the developing world. I am ever aware of the gross disparities and have difficulties rationalising the lives of the materialistically "contented" of the developed world to the millions of people who cannot even feed themselves on a daily basis. I again focus on my students, all students, in fact all citizens of the over-developed world who need to experience for themselves the lived lives of those much less fortunate than they. Only by exposure can attitudes change. Change needs to happen, perhaps by starting with small numbers there will be “converts” to a more open acceptance of less materialistic viewpoints/lifestyles.

Others have written about the value of reflection on activities outside of one’s comfort zone, particularly cross culturally. Bertaux’s (1981) position was that biography "uncovers the vast differences in social, economic, cultural, structural, and historical forces that shape, distort and otherwise describe life” (p. 4). Coffey and Atkinson (1996) stated “narratives are not 'naturally' occurring in that they are shaped, formed, and told according to connections and cultural understanding” (p. 80). RTA, as derived from biography, is a type of interpretive sociology as it “...studies real people who have real-life experiences in the social world” (Denzin, 1989a, p. 14). What is special is the individual processing that accompanies RTA.

RTA has been used methodologically for over eight decades. It was incorporated into sociological academic programmes in the 1920's and 30's in Europe and the USA. RTA fell into disfavour as the more scientific and technologically driven positivist model emerged in applied scientific disciplines. Social scientists bowed to more controlled observations and objectivity of the "pure science” of the day (Denzin, 1989a).

RTA experienced a renaissance of sorts in the late $20^{\text {th }}$ century, as interest in the "sociology of emotions" became prominent. Emotions could not be accurately measured using strict experimental processes. Hurlock (2003) directly addressed this issue, "In order to ask for emotions to be included in deliberation, there is a need to first question why emotions have not been included in deliberative experiences, particularly for positions that involve public deliberation, such as nursing practice” 
(p.116). Savage (2004) contended that the "significance of emotion" is well known in nursing, yet “...it is one of the more elusive areas of practice” (p. 25).

One aim of RTA is that readers of the subjective self-life-story are able to identify with what has been written, finding common ground with the material. According to Johnstone (1999) “... this in turn helps them to expand their own horizons of insight into and depth of understanding about their own lived experiences and the meanings they have attributed to these [events]” (p. 25). Another aim of RTA is to make the subjective world of an individual comprehensible, available and accessible to others. RTA is global in the sense that it uses multiple available sources for self-awareness. These sources may include selfreflection, views from others about self, literature that touches oneself and parallels intuition, and research from the social sciences, all of which portray a life event. Devereux (1967) said that it is crucial to understand what transpires within the observer if we are to understand what that person is observing, which has been validated by Behar (1996), Gordon (2002), hooks (2003), Lorde (1984), Rosaldo (1989), and Wolf (1996).

RTA is a research method not well known or used in nursing but is particularly well suited to the discipline. In the past nurses have used the case study method to discover, observe and learn about their patients. Now nurses/midwives, in their professional capacities can reflect and write about their own practices and further the art and science of their disciplines by critically expressing thoughts about unique experiences (Johnstone, 1999). By sharing the "reality” of practice as personally experienced, the qualitative view of that practice becomes richer with more sharing of knowledge gleaned among nurses/midwives beyond a particular locale. Dialectic themes later developed reflect another innovative use of RTA. This derivation is further addressed in Chapter VI. RTA has great potential for use in nursing/midwifery as the sociology of emotions and interpretive research is suited to nursing art and science. The following vignette reveals a montage about the art of nursing, family survival, and professional acceptance. 
The Prostitutes of

Pariahs of this city, yet visited regularly by the male population of all ages and marital status. The majority of the women are refugees from the civil war in the neighbouring country of They are escapees, these indigenous women and their children, their men folk dead, caught in the middle of civil strife. These are the fortunate ones, the ones that have crossed the border into yet how to support oneself and one's children. Opportunity knocks for these women in the guise of prostitution. Social services as we know them in the developed world do not exist. One must feed one's children and one must be expedient and pragmatic in order to ensure survival of the remnants of the extended family unit.

Women: adolescents, young adults, and middle aged all intent on keeping the surviving members of their families together no matter how one is shunned on visits to the town. These are marked women, intent in their goals of family survival, at whatever the psycho/socio/cultural consequences.

My students and I are very fortunate that we have been given permission to visit "the house" and talk with the sex workers; the owner even providing free cokes for us and the employees [although I have often wondered if the price of the soda was deducted from their wages]. We begin our journey early in the morning as we have a walk of approximately two hours each way. The priest at the compound where we are based has asked that we not use the jeep as it is emblazoned with the name of the parish and church; perfectly understandable.

The women are delighted to see us and have a break from work and tell us their individual stories. Each a tragedy in itself: Death, violation of human rights, intimidation, and racism, ad infinitum. They are mourning the losses of many family members, yet live in the present, and hope for the future. They are courageous women, charming women; we all feel so comfortable with one another; a camaraderie that women the world over innately share.

The topic turns to their occupation and their fear of "getting sick"; some of their customers "are so dirty". The women work in very tiny cubicles and ply their trade on small beds of wood covered with a thin mattress, sheet and thin bed covering. There is a small stand in the room that holds a basin of water for washing. They are allotted twenty minutes per client, and then must be ready for their next customer. 
The women prioritise their need to remain "clean" so that they will remain healthy and be able to continue working in order to provide the basic necessities for their families. They also are concerned about bringing home illness and disease to their family members.

We have brought soap for them to use after each client so that they can at least bathe their external genital areas. We work on a plan for them to utilise, and incorporate into their actual work, that of washing the external genitalia of their customers before sexual contact in the guise of "fun and foreplay". Twenty minutes is a short time, and each woman is usually in a position of submission, directed by her customer. Will each be able to integrate this seemingly simple practice? This will not protect the women against HIV or hepatitis, but they take comfort in knowing it may help prevent some lesser infectious diseases.

The prior vignette invokes how nursing does not occur in a social vacuum and as nurses we must understand the life circumstances of those with whom we work. We must promote unconditional acceptance of patients and their families. We must work with them at "their place and space". This may be an extreme example but nurses can identify their own perceptions and issues of scenarios that have been perplexing for them and realise that at some time or other each may be faced with challenging personal issues in their practices. For students, exposure to social issues can invoke many feelings, including the negative, and promote deep soul searching and learning about how people can be affected by situations beyond their control.

Reflective Topical Autobiography uses many steps and “...encompasses a deep inner contemplation (introspection) of the subjective self and its interpretive responses to lived experience” (Johnstone, 1999, p.25). Denzin (1989a) described deep contemplation of "turning point experiences” (p. 14) while Moustakas (1990) illustrated the process of personal “illumination” of a concept (p.29). RTA also enables the self (researcher) to return to original life stories adding new thoughts and insights through carefully constructed self knowledge and analysis (Johnstone, 1999). Johnstone (1999) wrote that one's own story is a story without end because there is always a "...leaving the way open for it to be revisioned and re-told as new understandings, insights and meanings are brought to it by the experiencing interpretative interactive self” (p. 25). 
The strengths of RTA include the ability of the writer to link the experiences of human existence with a reliance on rich subjectivity. RTA understands multiple self-realities as the individual interpretations of one's lived experience from multiple sources. (Denzin, 1989a; Douglass \& Moustakas, 1985; Johnstone, 1999; Moustakas, 1981, 1990).

The critics of RTA suggest that more empirical measures of quantitative science best move knowledge forward. Positivists who want definitive answers and conclusions to specific research hypotheses are less enamoured with such a singular and contemplative process (Denzin, 1989a; Ludwig, 1997). RTA is qualitative, reflective, thoughtful, and human. Yin (2003) defended single case study research methodology, which is similar to RTA (Johnstone, 1999) as a valid research methodology because it is “done in conformity with science's goals and methods" (Yin, 2003, p. x).

Role assumption with RTA means a researcher must consciously, purposefully and thoughtfully self reflect. "The power to change perspectives and view experiences in different ways” (R. Anderson, 1989, p. 436) was a powerful concept discussed in Kierkegaard on Ethics and Communication with Self (R. Anderson, 1989). Self reflection has the potential of altering one’s life (Kierkegaard, 1846/trans. 1941, 1851/trans. 1941). It takes time and willingness to challenge what is known and a desire to move to new realities. I have reflected very thoughtfully on the words of Kierkegaard (Anderson, R., 1989; Kierkegaard, 1846/trans.1941, 1851/trans.1941) and utilised his writings as a stimulus to enable me to pursue the RTA methodology. For me, the self researcher, this has meant honouring and rethinking my experiences, as well as questioning my motives for my chosen way of life.

Johnstone (1999) expressed her passion about RTA and its under utilisation in nursing stating “...reflective topical autobiography is an important research method in its own right but one which has yet to be fully explored and exploited as part of the overall project of advancing nursing inquiry and knowledge” (p. 25). To really think about what one thinks is seldom seen as legitimate research. It appears that RTA fits well with my thesis as it is a tool that explicates an intense life 
experience. For me the focus of my research has been my continuous indepth involvement with poor childbearing women in the developing world and the development of an immersion programme for North American baccalaureate nursing students in this same milieu.

\section{Autobiography/biography, and interpretive materials}

The use of biographical, autobiographical and interpretive materials as a source of data for research is not new. Sociologists and anthropologists have viewed themselves, individuals, groups, communities, and societies through their writings, art, music, photography, cultural practices and behaviours (Bertaux \& Kohli, 1984; Leininger 1985a; Oakley, 1992).

While each reader interprets the essence of the individual, many readers find "human” similarities in differences. Perhaps a way to explain the idea is to use the concept of grief. Even though grief is a universal event, it is very personally experienced, constructed, and socially constrained (Izard, 1991; Savage, 2004). For example a child experiencing the grief associated with parental loss may regress, refuse to talk, or behave in unpredictable ways. A spouse losing her/his partner may grieve by completely retreating into her/him-self or by changing their way of being in the world. Yet another person may actually die of a "broken heart” secondary to grief.

The following is an example of grief that took place during one of my immersion experiences in the developing world. For me it was an exploratory study in grief that transcended cultures and also a common occurrence in the community in question. It was the "out of order" demise of a neonate, predeceasing its young parents. This story reveals the continual grief that had become an everyday part of my life in the developing world. It was an experience that was replicated over and over again in many cultures. It was a frequently repeated event in the everyday lives of parents who routinely lost a baby/child to conditions that don't exist or are treatable in the developed world. It solidified, for me, the harsh realities of the relationship of culture, health and poverty. It brought "home" the understanding of the morbidity and mortality of the under five years of age population, but it never 
eased my personal grief. It made me identify with the writings and stories of others, and their feelings of misery, pain and impotence over events in their lives.

We are affected in similar ways even though we may react differently. We react in ways that are affected by the way we perceive. We are moved according to how we interpret all of this and by what it means to us, whether it seems threatening or reassuring, disturbing or quieting (Salk, 1983, p. 94).

The following vignette is:

\section{Foetal Demise/Stillbirth/Open Ward/Father Cardboard Box}

This is a city of two million inhabitants; most of its citizens live in poverty. The city hospital maternity unit is always bustling with activity; only the indigent come here to have their babies. The ward is completely filled and many beds are occupied by two post natal mothers simultaneously. Forty-two beds, twenty two on each side, neatly lined up, no partitions exist that afford any visual or auditory privacy. Those who bring their own bed linen are few, the rest lie directly on the plastic mattress covering. In a bed directly next to the nursing station is a young mother recovering from general anaesthesia, her stillborn neonate placed next to her; she, as yet unaware of his demise. Her husband is allowed in for a few minutes to sit next to her, and then is sent out to find a cardboard box so that he can take his dead son for burial. The mother is not afforded the opportunity to touch, caress, and talk to her baby. He is soon placed in the cardboard box which is too big to fit well, and as the father leaves the unit you can hear the remains of their son shifting as the box changes position. It is unwieldy in the father's arms.

When reflecting on the scenario described in this vignette I picture the parents, the father's eyes full of pain, and the mum dulled by the effects of her anaesthetic. I remained out of sight to the family yet clearly visualised the overwhelming grief of the father. I think about the developed world and parents and the expectations of a "perfect ending to a perfect pregnancy". If the results are not stellar blame is usually ascribed externally. In the instance described in the vignette the parents are poor, illiterate, and without a knowledge base of what has just happened and why. The father was urged to hurry and remove his dead baby from the unit; he has no perceived control and he never did receive any information from hospital personnel. When students are exposed to life experiences such as this they are fundamentally opened to a viewpoint that is broader than before. Once opened, 
students question the very nature of life. I believe this to be my "calling” to expose people to life as it exists, not the gilded version seen in the USA.

Several forms of auto/biography are commonly recognised, including the life histories of individuals, edited autobiographies, personal diaries, biographical sketches and historical research. One example, in nursing, includes the writings of Florence Nightingale (Fest, 2003a, 2003b; Nightingale, 1859/1980). Nightingale’s (1859/1980) autobiography gave us nursing knowledge that remains as pertinent today as when it was written. She wrote about sanitation, infection control, the advantages of fresh air and sunshine and the disadvantages of clutter and noise in the healing process. One can make comparisons with these differing methods of life writing. RTA is similar to these methods but is further refined, honed, and interpreted in the depth and focus of the exploration of a particular "turning point" in one’s life (Denzin, 1989a).

Of general autobiography, Langness and Frank (1981, pp. 88-89) stated:

...we hear the voice of someone putting us in touch with the experiences, thoughts, and feelings of another's life. The words take shape as images in our minds, as in the unfolding of a drama or the sudden moments of illumination in a poem. ...step by step, we distill that person's essence, weaving our characterizations around themes provided by the text. ... our understanding of the actual life depends upon...the images evoked.

Documenting life histories as "life writing”, has been an accepted form of biography for the last two hundred years (Johnstone, 1999; Kadar, 1992b). Kadar (1992b) aptly described it as:

Life writing is the most flexible and open term available for autobiographical fragments and other kinds of autobiographical seeming texts. It included the conventional genres of autobiography journals, memoirs, letters, testimonies...in earlier definitions it included biography. It is a way of seeing literary and other texts that neither objectifies nor subjectifies the nature of a particular cultural truth. (p. iii)

Johnstone (1999) wrote about forms of biography: the individual case study of the other, the autobiography, and RTA. RTA, as mentioned previously, focuses on "An account of the lived experience of the self that advances shareable understanding of common human experience” (p. 25). Allport (1942) discussed 
autobiography as a significant method of studying personal experiences. Defined as “...capturing 'turning point’ experiences’” (Denzin, 1989a, p.14) and/or “...existential moments” (Denzin, 1989a, p. 14), RTA provides insight (Moustakas \& Perry, 1973) into the life of a given person by serving “...the revelatory purpose of making the world of lived experience of that person directly accessible to others" (Johnstone, 1999, p. 25).

While the case study of one (Yin, 2003) is similar to RTA because it “... focus[es] on a contemporary phenomenon within some real life context” (p. 1), RTA is more deeply reflective and contemplative. The case study of one augments the understanding of the "other”, be it individual or group (Yin, 2003) by exhaustive and thorough study and analysis, yet relies on an outsider's viewpoint. Yin (2003) discussed the case study of one as being important, unique, justifiable and capable of bringing new knowledge to the social sciences despite rather harsh criticism. The detractors of the case study of one believe that the method lacks scientific rigour (Johnstone, 1999; Yin, 2003), a critique generalised to RTA as well. RTA uses multiple data sources to avoid this perceived weakness; multiple types of "rich" descriptive data provides rigour and depth to observations and experiences (Krefting, 1991; Lincoln \& Guba, 1985). “...the symbols and images that people use in telling about their experiences...give [further] substance and texture to the very lives they lead” (Langness \& Frank, 1981, p. 83).

Literary criticism as an auto/biographical technique has been in the forefront of inquiry into distinctly human issues. Spearheaded by feminists (Frey, 1993), black writers (Gates, 1993), ethnic literature, reader-response theory and poetry and post-structuralist work provide clear insight into how people have assigned value to their lives through exploration (Bertaux \& Kohli, 1984; Freedman, Frey, \& Zauhar, 1993).

I am especially struck by the auto/biographical critical confessions of Kanhai-Brunton (1993) and Lim (1993) and their writings on the effects of colonisation on individuals, groups, and societies. Their writing really strikes a personal note as the two authors poignantly describe the lives of people who have been colonised. Colonised people live(d) their lives on a daily basis juggling the 
cultural mores of two or more cultures, caught in between, and mostly excluded from mainstream life by the majority culture; it would be like living one's daily life on a tightrope. Colonised peoples are at the lowest socioeconomic rungs of most societies with educational attainment levels that are much lower than the average person. Colonised cultures confront substandard health care that is often not culturally congruent and many suffer with problems of multiple addictions, obesity, and chronic health issues (Huff \& Kline, 1999; L. Morris, 1999; Waldegrave, 2000). In some societies this cohort of people are not even identified as human. At more than one of the venues the indigenous population was constantly referred to as subhuman. The words spoken by professional health care providers were profound and for people who experienced these remarks, perpetrated by a supposed caring and healing professions, the results were humiliating. These feelings were also expressed and acted out by the local populace. It was not unlike the following quotation from the novel about the siege of Ladysmith, South Africa during the Boer war.

It amazed her, the extent to which the whites failed to see her as human. It was not even that they thought her nearer to the beasts, the oxen and mules; it was they failed to see her at all. To them, all blacks, were invisible, and silent too (Foden, 1999, p. 125).

People who are demeaned and dehumanised are often characterised and represented as having animal like qualities or as four legged animals (Curtis, 1971; Katz, 2005). Further, “ “...if a group does not seem to react in accordance with our conceptions of 'human nature', its behaviour is often depreciatively defined as 'inhuman or bestial’” (Devereux, 1967, p. 159).

On a personal level my older daughter, a Sioux Indian, was adopted at the age of five and a half weeks from an Indian reservation in South Dakota. She has been trapped across her lifespan due to Foetal Alcohol Effects that has impacted every facet of her being. Her biological mother died at the age of twenty-four, inebriated, during a physical altercation. My daughter's biological mother was one of twelve children of whom eight died before reaching the age of thirty. All died secondary to violence in their lives and their very tenuous and marginal position in society. It could be said that select nihilistic policies of the United States 
government toward Native Americans have had tragic and long-term effects. My daughter remains an outcast of society.

In The Intimate Critique-Autobiographical Criticism (Freedman, Frey \& Zauhar, 1993) multiple essays and stories were authored by those who were invited to submit "their" stories about their reality, in the forms that they wanted to present for publication. The editors (Freedman, et al.) felt that writing should be "personal and passionate” (p. 2) and should capture each individual's subjective feelings and world view rather than be “objective and impersonal” (p. 2). For each of the narratives the voice of the writer was clearly central to his/her chosen topic and the perspective of deeply felt personal experience and reality was the core and essence of the human experience. These are important concepts because writing subjectively illuminates the world of the "personal" and is significantly reflecting upon the "essence" of an individual and her/his world and world view. Readers, in turn, may then identify with feelings, thoughts, and events in their own lives as they are triggered, through reading the material. The following vignette, as it is so very personal, again triggers an emotive response for me.

Revisited

It is the monsoon season in India. August 1999. We stand on the streets of [name of city] hoping, not in vain. Looking for the maternity home where my daughter Gita was born. A city of destitute millions!

My daughter was one (but one) placed in a basket in the street for collection by an orphanage, a non governmental, non religious affiliated small facility where infants and young children who have been abandoned (many placed in prisons to be cared for by women inmates as the sheer numbers of abandoned babies and children preclude them being sent to government institutions) find a safe, caring and nurturing environment.

Four hours of searching with a taxi driver who knows the streets of this sprawling city brings us to the nursing home, a sign on the street proclaiming its name. We alight from the cab; I take a few pictures. 
Now it is time to climb the steps, the walls musty, caked with mildew, the paint chipped, and cracked and the stairwell neatly swept and clean of debris. As I mount the concrete steps my emotions are at a pinnacle, my daughter bounding up the steps; the full meaning of this assignation lost to a thirteen year old budding teenager brought up in the developed world.

I, as a midwife, who have worked for years in the developing world, know the pain of childbearing women. I think of the pregnant girl/woman who thirteen years ago ascended these very steps; her circumstances beyond her control; her culture and/or her economic status dictating her chosen path.

We enter the clinic and are greeted by an array of personnel who look questioningly at the white woman and the brown child. Someone is called who can speak English; I tell them my mission. Soon an employee who has been working there for many years is brought forth; she tells her story.

Young girls from surrounding villages and the city itself, women who simply cannot feed yet another baby, come here to have their babies and relinquish them; this is not the formal relinquishment known in the industrialised world. They birth and then disappear, surnames are pseudonyms, no questions asked. For many birth is induced, as the pregnancy is just becoming visible; the sari no longer able to hide the growing uterus, and mothers know that their daughters will never be marriageable, and they must act now. Girls initiated into the sexual act not understanding what they are doing nor knowing any of the implications or consequences that will ensue.

We are lucky enough to find the record book of Gita's birth; her biological mother has (had) an induced third trimester abortion. It is the only birth on the particular day in question. We cannot pursue this further, even if we had accurate information, as this would mean social disgrace and ostracism for the woman who gave birth to "our" daughter.

My memories take me to the airport in San Francisco surrounded by friends and my older daughter, Joey, which is another story to tell. A basket, a baby at four and a half months weighing 4 kilos, a long skinny body, with six strands of hair at the crown. When people, waiting for their flight realise that this is a uniting of mother, child and sister they extemporaneously begin to applaud and congratulate us. Serendipity!

I reflect, once again, on the dictates of society, gender roles and all its implications. Students need to understand how the formal institutions of society 
affect us all, though in different ways, dependent on the circumstances of birth. The following section talks about other emotive happenings in people's lives and how each of us is shaped by our experiences.

The auto/biographies of people with affective mental disorders are among the most compelling portrayals of the many challenges that life presents. Church (1995) in her doctoral thesis, Forbidden Narratives, provides a critical auto/biography of herself and others as survivors of the mental health system. Church wanted to "break out of the objectified modes of thinking and writing” (p. 139) about mental illness and also challenge the way people with mental disorders are treated by society. Her poignant story portrays the mentally ill as pariahs in society who, indeed, did not understand what their own needs were. As a sociologist she was ambivalent about writing "proper” sociology versus subjective sociology. As she continued with her writing, her beliefs, and that of her academic and personal support systems applauded her “subjective/objective connections” (p. 128.) as uniquely insightful. Church was writing and exposing the challenges that the mentally ill face in society, and at the same time challenging the traditional academic form of objectivity. An auto/biography, such as this, can be a source of abundant information that provides new understandings of mental illness (Sommer \& Osmond, 1960).

Other biographical and auto/biographical materials have been found in the stories of childhood survivors of incest. The word incest was scarcely whispered before the 1960's and survivors were relegated to silence by the implicit dictums of society. After that decade survivors' subjectivity and the end of silence prevailed as stories were published in graphic detail. This led to changes in the law as the many victims of childhood incest finally revealed their deep, dark secrets (Williamson, 1992).

Anthropologists Watson and Watson-Franke (1985) were among the first to express the concern of "losing someone" in a quantified and controlled scientific process; their pleas were to restore “...integrity and dignity to the individual in the face of the scientific onslaught...” (p. ix) and maintain rigour without loss of the voices of the people therein. 
Yet another forum of interpretive research is to study produced media such as films or plays. Ellis and Flaherty (1992) in their introduction to Investigating Subjectivity discussed Ingmar Bergmann’s classic 1966 film Persona as representative of people’s true lived experience, the “...real world..., a world characterised by multiple meanings and ambiguities” (p. 7). In the film a woman is portrayed as rejecting her young son yet the issues were far beyond that as she met another woman and a relationship developed and one woman mirrored the other and it was difficult to tell who was who, and what truly represented the "reel" and "real" realities. This is significant because the film paralleled real life in its multiple complexities and interpretations.

Lived experience is seen as interpretive (Polkinghorne, 1982, 1988; Davies, 1992; Denzin, 1994a; Kadar, 1992a, Taylor \& Bogdan, 1984) for those experiencing a phenomenon as well as those writing their commentaries about the experience of the other; the subjective being the important component. Two examples of interpretive research were evident in two popular Hollywood movies made about the life of a North American matriarch, Dr. Lillian Gilbreth, in the early twentieth century. This was a unique woman who became head of her large family with the unexpected death of her husband. She had to assume the financial and educational responsibilities of their many children. The woman was very well educated, which was uncommon in that era and even though it was a glossy Hollywood production the film, Cheaper by the Dozen, challenged traditional gender and economic roles within families. Graham (1992) read the actual (archival) personal writings of the matriarch of this family in order to deconstruct the image that was promulgated by the motion picture industry. By reading the central character's own version of the events in question, Graham found significant departures from those portrayed in the cinematic versions. She viewed the work from a perspective of feminism and an entirely new interpretation of what the woman and her family experienced. Graham stated that intra textual analysis and post-modern realism provided an interpretation and viewpoint in striking contrast to that of the films.

One difficulty with biography, auto/biography and interpretive research is the issue of subjectivity and emotion; Ellis \& Flaherty (1992) stated that subjectivity 
has long been treated as the stepchild of sociological scientific research. They said that sociologists felt revealing emotions or becoming intensely focused on the subjective experience with being human was not considered scientific. It appears that Ellis and Flaherty felt the study of emotions was outside traditional sociological confines and needed to become mainstream in sociological research. Emotions were studied peripherally, however, in the sense of separating the participant from the actual lived experience, by “transforming emotional experience into models of rational action” (Ellis \& Flaherty, p. 2) rather than capturing the real expression of feelings. This was, then, in an isolated manner, the sociologically constructed reality of human life, sans context, that did not accurately describe true emotions. Goffman (1959) worried that dealing with emotions in this manner only provided a superficial view of a person's feelings, the exposed public self. Sociological tools used during the time failed to capture emotion. An example was the questionnaire, with fixed choices and forced answers.

Ellis \& Flaherty (1992) attempted to explain that research methods that were not subjective in nature were too limiting and confining. Commonly raised issues included failure to capture feelings, an inability to describe relationships, the ineptness of categorising unique experiences using coding categories and lack of attention to body language. Data were placed in the researcher's (pre)determined categories that gave "privilege" and power to the researcher but separated emotions from other subjective categories such as the body, reasoning, physical phenomena, occurrences, and experiences. Additionally influencing factors included gender, culture, circumstances, at the time of the research (for the individual), and past personal history. Cancian \& Gordon (1988), Ramsden (2002), Stearns \& Stearns (1986), and P. Stevens, (1989) believed that society and history affected people's lived experience. A related problem, when a researcher uses the autobiographical method is “emotional exhibitionism”. Rosaldo (1989) maintained that this view was directly related to the early researchers, who were rich upper class white men who contained their emotions within and felt that feelings should be totally private.

Yet another issue with auto/biography is multiple "forms of data”. Autobiography can take its shape and structure from one of three techniques: one's 
complete and all-inclusive life, an abbreviated version of one's life, or a particular segment of one’s life that will hold meaning for others (Berg, 2001; Denzin, 1989a; Powell, 1985). A complete version provides rich context but is cumbersome. Using an excerpt or abbreviation of the life of the writer, one is opening oneself to the similarities of others and their experiences for the purposes of comparison, constructing links and furthering the insights and understandings of the common human condition. Key elements, emotions, feelings or content may be omitted. Use of a particular segment may richly portray that phenomenon but miss links to other life experiences and embedded meaning (Berg, 2001; Denzin, 1989a). In this instance the doctoral thesis will enable me to explore inquiry related to a particular activity, that of working as a midwife in many countries. The auto/biography of choice is RTA, as one remembers that RTA has been influenced by life experience and the "sociology of emotions" (James \& Gabe, 1996). It is the emotive, the affective realm, that is deemed of significant importance as emotions go beyond traditional physiological and psychological realms. We, as human beings respond to symbolically transformed objects as emotions, which then influences all communication processes as well as role functions and actions.

\section{RTA as the method of inquiry}

To review, RTA as a qualitative research methodology is particularly well suited to nursing because it enhances knowledge by endorsing and supporting the use of self as a research tool, linked to professional experience. RTA also promotes subjectivity to further the art and science of the discipline in order to create greater understanding of the similarities and differences of lived experiences. In RTA one must expose the self to scientific scrutiny and rigour, to personal exposure of one's true feelings and motivations, and open to the world one’s personal psycho/social milieu.

I feel that my professional life's work in nursing and midwifery is important and needs to be carefully reviewed and considered using RTA. I believe the issues that I have dealt with should be ruminated upon for new awareness and clarity. Perhaps, seeds can be sown, through awareness and common understanding that will 
be positively planted, nurtured, and grown. I will reflect on an extensive experience base in nursing and midwifery across the globe.

As an educator, I have been very fortunate to be able to immerse myself with nursing students in a wide variety of geographic locations in order to fully promote experiential learning. My stated aim has always been to nurture an expanding world view for students and promote growth and understanding of professional nursing and midwifery beyond the confines of the United States. I also write as a midwife, who has heard the voices of those forever silenced: the neonates, young children, and mothers, victims of circumstance through the vagaries of place of birth and status in society. Those voices, too, demand further rigorous contemplation. What were they telling me, telling us, about nursing/midwifery practice and life?

RTA has been chosen as my vehicle to frame this thesis. I had been directed to the seminal article written by Johnstone (1999) and read and re-read it many times trying to fully comprehend and digest its many meanings. I finally, and fully, realised that this method would allow me a new way to better understand my own life's work. This method uses interpretive research strategies, a clear valuing of emotion and utilises select principles of heurism.

\section{Heurism}

'Heuristic' comes from the Greek root word, heuriskein, and means “to discover or to find” (Moustakas, 1990, p. 9). For Moustakas heurism, "refers to a process of internal search through which one discovers the nature and meaning of experience and develops methods and procedures for further investigation and analysis” (p. 9).

Heuristic inquiry, a form of phenomenology, is greatly affected by personal engagement and involvement. It is the researcher herself who is, through her knowing, the tool, the instrument that undergoes indepth analysis (Moustakas, 1990); “...this challenges traditional scientific concerns about researcher objectivity and detachment” (Patton 1990, p. 73).

Another piece of the puzzle is Holstein and Gubrium's (2000) work that discussed the use of interpretive fieldwork, subjectivity, in order to understand the 
significance of what is taking place. For my research this means daily-lived lives, explicit details, familial and community significance and implications, and roles and functions of individuals within their society. Harry (1996) also emphasised that comparison is needed “...beyond the immediate circumstances of the local setting” (p. 65).

Central to this research process is the self who is ever present and continues to evolve as she/he delves deeply into the phenomenon under study and gleans and “experiences growing self-awareness and self-knowledge” (Moustakas, 1990, p. 9). Two of the main tenets of this process are to be continually fully engaged and receptive to new discoveries within one's personal experiences. It is also important to combine one's knowledge and lived life to broaden awareness in order to expedite the heuristic process so that one can investigate one's world very intimately.

Reflection is a very important aspect of heuristic research. Taylor (2000) maintained that the actual nature of reflection based on one's life allows a new level of being and knowing, whether professional or social experiences. Taylor further stated that there are many ways of knowing such as learning from all forms of creative expression including dance, art, song, or poetry. Moustakas (1990) utilised the tools of contemplation, meditation, and intuition in viewing creative expressions to better understand a life-changing epiphany.

Reflection can be classified as a logical, efficient and organised research approach of looking at daily actions and events and making sense of the meanings of occurrences that then act as “...catalysts for self-awareness and personal growth” (Taylor, 2000. p. 5). Reflection is a “...reconstructing experience used ... in order to ...describe...experience, to work through the attitudes and emotions which might colour ...understanding, and to order and make sense of the new ideas and information...” (Boud, Keogh, \& Walker, 1985, p. 11).

Schön (1983) considered "reflection-on-action" as a key element to the practice of any profession. Professions are usually defined as autonomous, intellectual, practical and theoretical, motivated by altruism, and guided by internal organisation (Craven \& Hernle, 2003). Schön (1983) defined the reflective element as a phenomenon in which an individual is trying to work through her/his own 
professional endeavours to understand and make sense of what has transpired in an event, or series of events. By recalling all the actions, verbage, and actors, in other words a complete "instant replay", professional practitioners carefully reconstruct the scenario to gain new understandings and further insights. Schön (1983) further stated that though “...some puzzling, or troubling....phenomenon with which the individual is trying to deal with [sic] arises, the reflection process has the power to clarify, amplify and describe something not previously understood (Schön, 1983, p. 50). Time is not a factor and clarity of the phenomenon can be ruminated upon long after the phenomenon has taken place as meaning can become even more intense with further reflection and scrutiny of the phenomenon (Moustakas, 1990). Schön (1983) states that when one reflects-on-action “...he [she] becomes a researcher in the practice context” (p. 68).

Smyth (1986) says: "There has to be a transformation from a position where scientifically derived knowledge is deemed superior to a circumstance in which artistic and intuitive knowledge may be equally appropriate...” (p. 7). To Dewey (1916), reflection represented bringing together the whole of the experience by linking the separate entities. To others, such as Boud et al. (1985) and Hertz, (1997) reflection meant examining one's own thoughts by profoundly and deeply thinking about the experience(s) in order to thoroughly understand what transpired and what one learned from the experience. In summation "reflective learning is the process of internally examining and exploring an issue of concern, triggered by an experience, which creates and clarifies meaning in terms of self, and which results in a changed conceptual perspective (Boyd \& Fales, 1983, p. 99).

The heuristic process is a way of knowing, of being informed. It is the retrieving of information that is held in the consciousness. Knowing is achieved by personally utilising multiple methods in order to have access to that which may be hidden in the psyche and experience. The ultimate aim of this research process is investigating one's own personal human experience. Whatever it takes to trigger access to one's innermost thoughts and being has particular value in human knowing. What surfaces must be thought of as part of the puzzle that gives clues to more fully understanding that elusive something and what it might mean. In this way 
there is both personal development and intellectual growth. The process involves constant self-searching, self-discovery and self-dialogue, with the significance and importance coming from an ever growing amplification of inner self-awareness and discovery. In heurism one must be consumed by the issue(s) and consistently search within for meaning. Whatever is in the consciousness is considered necessary to focus upon, via inhabiting and residing in these thoughts with one's full being in order to begin to perceive and understand the possible implications of the significance of the phenomenon. By constantly endeavouring to enrich one's self, by intensely understanding the experience under scrutiny and developing it into a research paradigm, new realities can be reached (Moustakas, 1990).

Moustakas (1990) writes that in this process of conscious reflection of phenomenon it is not uncommon to challenge or even doubt one's own thoughts about the subject that is being studied. What becomes important is personal persistence until one increases their knowledge about the issue/research in question. It is the "I" that is intently involved in this process. It is the subjective viewpoint by the one who is continually searching to expose and reveal to her/him self what truly matters. The “...qualities, conditions, and relationships that underlie a fundamental question, issue, or concern” (Moustakas, p. 11) then arise, which are directly related to a crucial human event. One becomes so involved in this process that images, dreams or visions about the issue may be found along the path of discovery. One may discover new things about oneself, and one's relationships with others may be seen in a new light. Being open to change and further discovery of self are inherent in this process. There is a connection to the external world and what one sees, hears, touches, feels, and smells is part of the reflective process within. It is being able to perceive anew that which may be the mundane of daily life. Moustakas said: "When I illuminate a question, it comes to life. When I understand its constituents, it emerges as something solid and real” (p. 12).

The soul of heuristic research is “... the investigator's internal frame of reference, self-searching, intuition, and indwelling” (Moustakas, 1990, p. 12) thoughts. Moustakas emphasised that as one studies an issue, it can induce pleasant thoughts and even very disquieting and unsettling ones. It is going down a specific 
path of inquiry with the knowledge that one is entering a potentially unsettling unknown. One must trust oneself, as indeed it is the path of self-discovery and one is utilizing one’s own powers of observation, feelings and intuition. This becomes the basis of the validity for "clarifying a topic, question, problem, or puzzlement" (Moustakas, p. 13).

One manner of heuristic approach is the analysis of self-talk. Vocate (1994) defines "self-talk" as dialogue with the self “...the self is both the source and the object of the interaction” (p. 7). Mead (1967) called this 'self-awareness' and expanded the term to encompass 'reflective consciousness'. This form of communication can be either silent or vocalised. Moustakas (1990) called this selfdialogue. Pelose (1989) discussed intrapersonal communication as “communication with/within oneself and involves introspection about one's thoughts, perception, and beliefs” (p. 135). Intrapersonal communication can be both expressive and constructive by informing and generating meaning (Johnson, 1994,) and gives revelatory meaning to oneself via the intrapersonal process. Daily, as human beings we talk to ourselves and these “...self-directed monologues” (Johnson, p. 176) may be audible or inaudible. I can readily identify with intrapersonal dialogue as it is a “technique”, with which I am completely comfortable. What is imperative and crucial, is to understand and explore the meaning, value, purpose and depth of what the self really has to say.

I consider myself a "subjective knower”; one who “...depends on their [my] personal experiences. These 'knowers' believe and depend on their own inner voices and inner feelings” (Meleis, 1997, p. 176) to know the truth. The subjective knower is not well expressed, flowing, or effortless in "articulating the processes used to arrive at the knowledge" (Meleis, p. 176) yet has "the wisdom that holistically looks and explains complete situations” (Meleis, p .176). Belenky, Clinchy, Goldberger and Tarule (1986) further stated that "truth, for subjective knowers, is an intuitive reaction, something experienced, not thought out, something felt rather than actively pursued or constructed” (p. 69).

As a researcher I am passionately attracted to Moustakas' (1990) heuristic premise. It is almost as if it is a fatal attraction; a junction that I have reached; a 
scrutinising of, who I am and why I do what I do, in that I was impelled to examine, introspectively probe and investigate my modus operandi (Moustakas, 1967, 1972, 1975, 1990). Moustakas (1990) is compelling and motivating in his descriptions, theory building, and reaching out to others by tantalising, obliging and persuading one to self-examine. I therefore chose to pattern my process of a life changing experience on Moustakas’ (1990) model.

I took on this project to reveal and revel, learn and understand, explain and expunge, through giving "me” permission to truly explore myself and my motives and purposes as I journey through life professionally and personally. I gave to myself, when I committed to this project "the gift of self to create” (Moustakas, 1977, p. 48); this was a very new "space” in my life and it caused a very "significant awakening” (Moustakas, p. 43).

It is imperative in heuristic research that the researcher has direct auto/biographical experience and connection with the phenomenon under investigation. I have been working in the developing world since 1971 and now must scrutinise my reasons for returning again and again to work under adverse conditions, immersed in poverty and tragedy as a daily occurrence of life for the actors; the childbearing women of poverty of the developing world. I also must look at my need to expose nursing students to this same milieu. I feel that I am personally committed to unravelling this mystery by committing to delve deeply within myself. I hope to discover and explicate that which has been elusive to me, hidden to me, "Why do I go? What do I yearn for? What do I see? What magnetic forces pull me back time and time again?”

I realise that the heuristic process is a very difficult one to undertake, as it is very challenging, yet at the same time motivating and thought provoking. The process involves my telling my story (self-talk) over and over again to myself, my internal auditor. "Heuristic research is a demanding process. It requires "rigorous definition, careful collection of data, and a thorough and disciplined analysis” (Frick, 1990, p. 79) with the responsibility solely on the researcher.

When I think of tacit knowing, which is an integral part of heuristic research, words such as: implicit, understood, unspoken, unstated, inferred, unexpressed, 
couched, contained, hidden, buried, come to mind. Polanyi (1966) called it “... a way to know more than we can tell” (p.18), and Benner (1984) talked about "knowledge embedded in expertise” (p. 3). Schön (1983) stated that we cannot always articulate this “knowing”, yet we do "know”, and Taylor (2000) stated “... actions guided by knowledge you may not be able to express” (p. 55), still guide those actions. Tacit knowledge is succinctly, and perhaps best, expressed by Polanyi (1962) as “...inarticulate acts of intelligence” (p. 95). Grene (1969) stated: “...discovery cannot be explained in terms of wholly formalizable, wholly, explicit knowledge” (p. ix). Returning again to Polanyi (1959), “The structure of tacit knowledge is manifested most clearly in the act of understanding. It is a process of comprehending: a grasping of disjointed parts into a comprehensive whole” (p. 28). Intuition is another piece of the heuristic theoretical puzzle and Moustakas (1990) aptly described intuition as follows “...makes immediate knowledge possible” (p. 23). Perception is involved in intuition and one observes over and over again until the complete, the whole, emerges as the certainty of the reality, the truth (Moustakas, 1990). Polanyi (1969) stated that intuition is vital to scientific enquiry; "intuition is a skill, rooted in our natural sensibility to hidden patterns and developed to effectiveness by a process of learning” (p. 118). In the heuristic process intuition is used repetitively and the researcher continually refines her methods and understandings in her pursuits, as she seeks the truth. Using intuition the researcher can continually question herself as she delves deeper and deeper into the vortex of the phenomenon she is researching, as she finds herself completely immersed and enmeshed.

Another concept used by Moustakas (1990) and Polanyi (1962) is "indwelling”. In the heuristic model this means a focusing that endorses and sanctions a thorough and intensive self-directed scrutiny of a segment of an experience or the experience in toto. It is an essential element in the heuristic process that requires internal focusing coupled with self-talk. Douglass and Moustakas (1985) stated that it is inner attention, and getting in touch with the precise meaning of the experience, via clearing the mind in order to get to the awareness and insights. 
Heuristic inquiry connotes the path to discovery through personal inner inquiry; it means flexibility, creativity, openness, self-discovery and innovativeness on the part of the researcher, the self (Frick, 1990). It means the researcher has direct experience with the "problem" or question and Douglass and Moustakas (1985) stated that “...self-experience is the single most important guideline in pursuing heuristic research” (p. 46). Heuristic inquiry has all to do with a personal and passionate, inquiry. It “....is the search for the discovery of meaning and the essence in significant human experience” (Douglass \& Moustakas, p. 40).

Douglass and Moustakas (1985), Moustakas (1990) and Johnstone’s (1999) model of RTA share many of the same steps. Johnstone’s (1999) model follows Moustakas's (1990) framework, even though some of the wording is changed. They both share: the initial engagement, immersion, incubation, and illumination. It is at this point in RTA that Johnstone (1999) advocates the formal writing. As I am framing this entire thesis utilising all of the steps of Moustakas (1999) I will now set out an explanation for each step. I will also include a section on Johnstone's (1999) model of RTA.

Initial engagement: The most important activity of this step of the research is to formulate my main concern, the passion that has consumed me for years in both my profession and avocation. The process is: self-talk and self-searching in order to come into profound contact with the research questions. It is here that I am fully engaging myself, and have fully engaged myself into my life experience(s) in order to clarify each research question. An important tool is reaching inward for tacit knowledge (Douglass \& Moustakas, 1985; Frick, 1983, 1990; Johnstone, 1999; Moustakas, 1990; Polanyi, 1962, 1966, 1969).

Immersion: The research questions, once defined, are honed, and fully explored. The focus is answering these questions by living, sleeping, devouring, and even dreaming, the themes, ideas, and pictures in the mind. It is reflecting again on the slides that I have taken over the many years; vignettes, which I have written related to the experience(s) that will need to be scoured; and examining the formal 
Table 2.1

The Moustakas Model of RTA

\begin{tabular}{ll}
\hline Step 1 & Initial engagement \\
Step 2 & Immersion \\
Step 3 & Incubation \\
Step 4 & Illumination \\
Step 5 & Explication \\
Step 6 & Creative Synthesis \\
\hline Note. From Moustakas, C. (1990). Heuristic Research. Newbury Park: Sage.
\end{tabular}

Note. From Moustakas, C. (1990). Heuristic Research. Newbury Park: Sage.

readings related to my question; what is it that impels me to return again and again to work with poor childbearing women in the developing world? I plan to talk to others in all walks of life about what they know of my experiences and solicit their comments and views and through undertaking interviews with the students who participated in the programmes. (Douglass \& Moustakas, 1985; Johnstone, 1999; Leininger, 1985b; Moustakas, 1956, 1959, 1966, 1967, 1972, 1975, 1990).

Incubation: This is the point in time where the researcher, I, have distanced myself from the topic. Moustakas (1990) stated: “Although the researcher is moving on a totally different path, detached from involvement with the question and removed from awareness of its nature and meanings, on another level expansion of knowledge is taking place” (p. 28). I think of this as dejá vu, or gestalt, when all of a sudden meaning appears “out of the blue”, eureka, that's it!

Illumination: Is the epiphany, the ultimate understanding (Douglass \& Moustakas, 1985; Frick, 1983; Johnstone, 1999; Moustakas, 1990), “...the door to a new awareness, a modification of old understanding, a synthesis of fragmented knowledge, or an altogether new discovery of something that has been present for some time yet beyond immediate awareness” (Moustakas, 1990, p. 30). This is what Frick $(1983,1987,1990)$ called the symbolic growth experience. He explained this 
hypothesis as a unique learning experience, often a profound one, for an individual. “This concept refers to those significant moments in life when we create personal meaning by symbolizing our immediate experience in the interest of heightened consciousness and personal growth” (Frick, 1987, p. 108).

Explication: In following through with Moustakas (1990), the next process is explication. The researcher once again employs all the prior processes that have been utilised to arrive at the epiphany “...in order to understand its various layers of meanings” (Moustakas, p. 31) and understand the themes and qualities of the revelation.

Creative Synthesis: The last step is the creative synthesis. This implies that the researcher "has mastered knowledge of the material that illuminates and explicates the question, the researcher is challenged to put the components and core themes” (Moustakas, 1990, pp. 31-32) into a written document be it poetry, art, or other representative form. It is at this point that Johnstone (1999), as well as Douglass \& Moustakas (1985), and Moustakas (1990) advocate writing the RTA. Johnstone (1999), as well as, Douglass \& Moustakas (1985) and Moustakas (1990) informed the user of this method, that there are no set rules for writing, "self-lifestory” (Johnstone, 1999, p. 29). Johnstone(1999) and Denzin (1989b, 1994) recommended the following considerations: in subjective writing one must be able to portray, and evoke, through the medium of words, feeling. Johnstone’s (1999) second point emphasised being able to tell the story with a very personal approach (Holstein \& Gubrium, 2000; Moustakas, 1975; Salk, 1983). “The personal story, especially, is being resuscitated as an important source of experiential data (Holstein \& Gubrium, 2000 p.103). Lastly Johnstone (1999) stated the self-narrative needs to be able to stand up to scrutiny and affirm writing in the first person about personal experiences.

Each of these steps is highlighted in order to describe the parallel research methods of Moustakas (1990) and Johnstone (1999). These philosophic organising concepts have been methodologically translated into specific research activities. As indicated in Table 2.1, the Moustakas Model (1990) of RTA will be used to direct the entire research project in order to answer the research questions. 


\section{Summary}

This chapter has examined RTA (Johnstone, 1999), and Moustakas’ (1990) heuristic model, and its relationship to biography and interpretive research. The focus of both is on issues of personal concern combined with one's own auto/biographical experience and deep reflection. RTA calls for self scrutiny and examination through reflection and one being compelled to address one's very essence through self examination. The heuristic process is a way of knowing, of being informed, of self searching, which can lead to personal turmoil as one thoroughly investigates, immerses, and engages oneself in the particular phenomenon under investigation. Through living, sleeping, self-talk, internal fixation, and deep passionate reflection an epiphany is reached that reveals to the self researcher new understandings and knowledge of self that lead to life changes. There are six steps in this process that one follows: Initial engagement, Immersion, Incubation, Illumination, Explication, and Creative Synthesis. This chapter has also reviewed the literature on the importance of acknowledging emotions in scientific writings. The next chapter will fully discuss the study design and methodology. 


\section{CHAPTER III}

\section{STUDY DESIGN}

"Our lives begin to end the day we become silent about things that matter" Martin Luther King, Jr. (Quotation, n.d.).

\section{Introduction}

The prior two chapters have set the stage for this study by addressing the immersion programme and the conceptual premises and circumstances in which this study was conducted. I will now address the actual methods and processes used to complete this project. This chapter will include the study design, review of RTA, the researcher's perspective, sampling process, interviews, setting, protection of human subjects, data collection, data analysis and rigour.

\section{Study Design}

A naturalistic (Lincoln \& Guba, 1985) qualitative methodology was used because more typical quantitative measures could not provide insight into the subject matter of this study. According to Lincoln and Guba (1985) “The instrument of choice in naturalistic inquiry is the human...” (p. 55). Qualitative research has been described as that which facilitates and creates understanding of the dynamic flow of lived lives. The use of textual material and description add to the richness of qualitative methods and serve to distinctly separate it from quantitative projects (Lauterbach, 2002; Leininger, 1985b; Ray, 1985; Morse, 1997). Leininger (1985b) states that qualitative research is extremely useful in order to actually understand what is "going on”, and the results increase the body of qualitative research knowledge. World view(s), value systems, mores, ideas, convictions, and thoughts add to the totality of experiences and actions (Leininger, 1985b), in other words, enhance the text with the richness of the data. "It is an approach in which the 
researcher explicitly participates in uncovering the meaning [of] experiences as humanly lived” (Parse, Coyne \& Smith, 1985).

Also the naturalist researcher realises “...the reality that situations constantly change and people’s realities differ” (Gillis \& Jackson, 2002, p. 216). This study used the technique of interviews for concentrating on informants' stories, the subjectivity of each, their point of view, and their reality. This ensured consistency by including students from each programme; the reality of each participant being unique as “...there is no reality except individual reality” (Moustakas, 1967, p. 40) and the programme taking place over many years and many different locations.

Implicit in the qualitative process is bracketing in which all preconceptions are held in abeyance. Bracketing is the process of isolating preconceived notions or ideas carried by the researcher in order to view the data purely (Gillis \& Jackson, 2002; Holloway \& Wheeler, 1996). A “pure” view of data allows themes to emerge without egocentric contamination. The process of reflective review is used to describe how knowledge emerges from the data in the form of the heuristic process in this thesis (Chenitz \& Swanson, 1986; Glaser \& Strauss, 1967; Morse, 2001; Schreiber, 2001; Stern, 1985; Taylor, 2000).

Multiple data sources were used to get a deep and profound perspective of meaning using RTA. This study used interviews as well as deep personal reflection as data sources. I thoroughly reviewed my extensive slide collection which triggered instant memories and insights; again the psychosocial pain was evident as I thought deeply about the mothers, babies, and infants. Diaries, field notes, my published articles, research articles, personal communications and experiences, presentations, lectures, memory, and my vignettes were also incorporated into the reflective process on the road to the discovery of the pinnacle experience. The interviews with the students were extremely important to include because they identified students experiences, feelings and emotions, not necessarily shared at the time of the immersion programme. A much deeper, reflexive and processed viewpoint was obtained during the interviews.

Data were analysed "looking for patterns, threads, tensions, and themes, within and across individuals’ personal experiences” (Clandinin \& Connelly, 1994). 
Consistent with post modern thinking, post modernists write of multiple realities, rather than one didactic truth, thus there exist many constructed "realities” related to specific phenomena. For this reason interviews, introspection and the aforementioned sources were utilised. The very essence of qualitative research is in the: "knowledge of the multiple contexts in which that phenomenon occurs" (Hinds et al. 1992, p. 35).

\section{Reflective Topical Autobiography (RTA)}

RTA, to recap, is an underused but highly beneficial research process and was selected for this thesis because it is a method that looks at complex human issues. RTA uses a rigorous approach to studying pivotal life phenomenon(a) and the ensuing enlightenment that follows. It is an auto/biographical method that focuses on subjectivity and makes individual understanding obvious, discernable, and noticeable. It increases "the search for meaning and increasing understanding of the commonality of existential human experience” (Johnstone, 1999, p. 24) as well as seriously addressing multiple realities and individual elucidation related to lived experiences (Coffey \& Atkinson, 1996). RTA “tells a story, but never claims to have told the 'whole story' leaving the way open for it to be revisioned and re-told as new understanding, insights, and meanings are brought to it by the experiencing interpretative interactive self.” (Johnston, 1999, p. 24). The methodology of biography is an integral component of this form of research.

As revealed earlier, I have chosen RTA as my methodology for this thesis because of its fit with my multiple experiences of work in the developing world and my very personal continuing search for my motives for repeatedly returning as well as the reasons for developing an immersion programme for baccalaureate nursing students.

Assumptions, explicit to RTA, include the valuing of auto/biography that focuses on one individual's lived experience that has as its aim the identifying human commonalities. "At the most fundamental level, people exist generically as human beings, and as such, share many features in common. They have similar biological drives and basic psychological needs and respond to the same sorts of 
social pressures” (Ludwig, 1997, p.91). Another assumption is that the purpose of RTA is to use one's own richly subjective experiences as a way of examining and promoting a better understanding of life's pivotal happenings. Another basic assumption is that one must explore, to the utmost, one's personal life experience(s) utilising multiple modalities to gain inner access to the workings of one's psyche in order to make sense of, and fully comprehend, a pivotal experience(s) and how one's life has been changed. Utilising RTA one must deconstruct, to minutiae, a crucial and critical life experience(s). Denzin (1989a) discussed deconstruction as having the following properties: it readily identifies prior concepts related to the event(s); it decisively decodes these concepts; it significantly probes human action and "it presents the perceived biases that are believed to exist specific to the phenomenon" (Denzin, 1989a, p. 66).

The next step, intrinsic to RTA is reconstruction through the process of deep personal reflection, readings, review of slides, interactions with others, and further self-exploration in order to arrive at a new reality related to the phenomenon and to the self.

\section{My Perspective}

I have successfully combined my nursing, midwifery, and teaching careers. I am telling my story, my metamorphosis, and evolving personage, predicated on my living and practising midwifery in a multitude of diverse communities across the world. Context was important as I sought to fully understand the reasons for my continuing work with poor childbearing women in the developing world and the reasons for taking students to work in this same milieu. My thesis is based directly on informant interviews and my feelings and learning about myself through my extensive interactions with clients, families, and communities. The capstone of my career is the sharing of this personal journey. From my perspective the "clinical" setting and the important peripheral data such as lifestyle, culture, economics, education, religion, family and government all interacted to affect my deeply reflective processes. Barnard, Towers, Boston \& Lambrindou (2000, p. 1) stated: “...in the real world, one is brought face to face with institutional limitations, human 
foibles, problems giving care..., and challenges of cultural diversity, among other things." The physiologic processes of labour are shared the world around but the psychosocial processes are shaped by the milieu in which we live. Throughout this project, I have used my own reflections and memories as a source of rich, thick data congruent with RTA. This is most easily seen in the vignettes that have been included. The vignettes best clarified the points I was seeking to make, and framed the dialectic that I have presented. The following vignette describes one very busy labour ward where I worked. This was a poor country and for those unable to afford private care, the local public hospital, was the only alternative for those women who lived in this small city.

Labour/Abject Fear

Screaming, tenseness faces contorted, eyes bulging and not focusing, bodies and appendages rigid. With each ensuing contraction the process begins again. Labouring alone, made to lay flat on one's back, two to seven other labouring women sharing a common room; each filled with abject fear. Family were made to wait outside in a waiting area, and they could hear the hours of anguish and screaming but were never allowed in to see their loved ones. The primigravida especially frightened as this was a very new experience; their preparation consisting of what their mothers had told them about "having a baby". The staff ignoring the sounds and pleas of the girls/women in their labour beds, attending to them only when they perceived (from the grunting sounds) that birth was imminent. At that time each parturient was ambulated to a common birthing room and manoeuvred herself on to a short narrow birthing table whereupon her legs were placed in stirrups and she would then wait for an attendant to give her directions, too frightened to do anything but scream.

The vignettes have been an important part of my reflective process and of gaining insight into the emotional impact my experiences have had on my life. As I wrote, and as I read them, the memories flood my psyche and say to me: People of the overdeveloped world really need to understand the often negative process of labour and delivery for the majority of the world's women.

In this study I have served as the research instrument through the use of myself as an observer and retriever of students' experiences in the immersion programme, capturing the essence of their experiences as they related them to me 
during their interviews. A major source of my data has been derived from these interviews with 10 students who participated in the programme. Students were immersed in varying cultural settings in the developing world. Gilles and Jackson (2002) stated that the richest data comes from immersion into a particular culture. Students were able to speak to their experiences as both observers and participants and were able to discuss their perceptions of the immersion in depth. Gilles and Jackson stated the participant observer "is considered to be an insider” (pg. 5), which provided me with rich student feedback upon which to reflect because "participant observers watch what people do, listen to what people say, and interact with participants” (LeCompte \& Preissle, 1993, p. 196).

\section{Participant Selection}

Following the institutional review processes, the identification of potential informants was initiated. The sampling process used in this study was both convenience and purposive. A convenience sample is utilising people who are readily available and who understand or have lived the experience of concern. Each informant provides insight into the process and has experienced the phenomenon. This method of sampling is very frequently used in nursing research (Nieswiadomy, 2002; Powers \& Knapp, 1995). It is also a method that uses time effectively, gleans the essence of the area of focus and provides contextual insight.

Purposive sampling was also used for a group of interviewees in order to include a diversity of students who knew about and partook in the immersion programmes (Creswell, 1998). I felt that a range of participants, including the younger partakers and also a male, would increase and expand information relevant to the immersion programme, thus a cluster of key informants were capable of describing the experience on which this work is based. 
The selection criteria for interviewees were as follows:

1. Participation in immersion programme offered by Humboldt State University Department of Nursing

2. Subsequent completion of undergraduate BSN programme, for those who were not senior students

3. A willingness to be audiotaped

4. A willingness to review the transcript in order to clarify salient concepts and ensure rigour

I attempted to interview at least one participant from each immersion programme and also a variety of age ranges of the participants. The table below describes the demographic details of the 10 students who agreed to be interviewed about their experiences in the immersion programme.

A formal letter (see Appendix A) describing the study and soliciting participation was sent to local former students who had participated in the immersion programme. Potential participants were asked if they were interested in being interviewed about involvement in the immersion programme, and if so, to please notify me by signing the letter of interest provided. The letter explained that the interview would be taped, each interview would last approximately an hour,

Table 3.1

Demographics

\begin{tabular}{ccllcc}
\hline Age & $\begin{array}{c}\text { Yrs. } \\
\text { Completed of } \\
\text { Major* }\end{array}$ & Venue & Gender & $\begin{array}{c}\text { Marital } \\
\text { Status }\end{array}$ & \#of Children \\
\hline 20 & 1 & Russia & Female & S & Nil \\
21 & 1 & Russia & Female & S & Nil \\
22 & 2 & Slovenia & Female & S & Nil \\
23 & 1 & Nepal & Female & S & Nil \\
27 & 3 & Philippines & Female & M & Nil \\
28 & 1 & Slovenia & Female & M & Nil \\
31 & 3 & Russia & Female & M & Nil \\
34 & 2 & Mexico & Female & S & 1 \\
43 & 1 & Dominican & Female & S & 1 \\
46 & 2 & Republic & & & \\
\hline
\end{tabular}

* Nursing major courses span three years 
though was not time limited and the transcriber of the audiotapes would only know the participant by their pseudonym (see Appendix B). The letter soliciting participation also included the statements that the participant would have the opportunity to review their transcripts for correctness, and be asked about the identified themes or patterns used in the thesis in order to ensure accuracy. A second letter, a consent form was mailed to proposed participants (see Appendix C). Each participant chose a pseudonym to ensure confidentiality. To further ensure confidentiality interviews were conducted in private and meticulous control of the audiotapes and transcripts were continually assured by their being placed in a locked cabinet with only one key that the researcher holds. Tapes will be returned to the participants at the conclusion of the study and transcripts will be securely kept for six years for possible use in further articles, professional presentations, or further data analysis. Participants were polled concerning mutually convenient times for the taped interview to take place.

The study was conducted using semi structured interviews with personal reflections. Data collection continued until the point of data saturation or redundancy. I was informed by my understanding of the methods described in grounded theory (Chenitz \& Swanson, 1986; Glaser \& Strauss, 1967; Schreiber \& Stern, 2001). Lincoln \& Guba (1985) defined redundancy as reaching a point where no new information is attained. Data saturation was reached when seven informants described consistent experiences, themes, patterns and categories. Because the goal of the research was to obtain rich descriptions on which to reflect, the additional three interviews were conducted to ensure rigour. A total of ten interviews were conducted.

\section{Interviews}

Interviews of former students who participated in the immersion programme were one modality of data retrieval. Self reflection and analysis added further clarification of embedded concepts. Through interviewing students and collecting their stories I was able to discover feelings, thoughts, perception of self and others, 
changes in the affective realm, professional and personal change, and changes in world view.

The study was conducted in Northern California where I live. The choice of venue for the interviews was an impartial informal setting. Tape recorders were set up in order to assure an accurate record of informants' views. The interviewee and the researcher were the only people present during the interviews and telephones were turned off so there would be no disturbances.

Each of the interviews lasted approximately one hour. At the beginning of each taped interview a preamble was read (see Appendix D) relating to confidentiality, use of the data, approval before its use by the researcher, and that the participant, could at any time stop the interview if he/she no longer wished to continue. Probes were used to guide the interviews.

One copy of each tape was sent to the transcriber in New Zealand who only knew the interviewee by their pseudonym and who had also signed a confidentiality statement (see Appendix B). The transcripts were returned to the participants for corrections or clarifications of salient topics. As mentioned previously the tapes will be returned to each participant at the completion of the thesis.

“At first glance, the interview seems simple and self-evident” (Gubrium \& Holstein, 2002, p. 3) but that is not the reality. There are myriad purposes for interviews and many "seemingly" minutiae that must be attended to. The environment, such as time of day, location, physical surroundings, and comfort are of prime importance as well as the psychosocial state of the interviewer and interviewee. Interviewing is purposeful, it requires focus, preparation, attention to verbal and non-verbal communication, and it is informed by theory (Murphy \& Dillon, 1998). An interview has structure in that it has a beginning, middle and an end and it is a particular type of dialogue exchange. One of the most important aspects of an interview is the realisation "individuals speak, listen, and make meaning from their own unique perspective. Two people may share the same experience and yet each perceive[s] it uniquely” (Murphy \& Dillon, 1998, p. 5). Gubrium and Holstein (2002) posited that individuals are acknowledged as significant commentators on their own experiences. During each of the interviews I 
listened intently by focusing on the verbal and non-verbal cues, body posture, and feelings that were being visibly portrayed by the participant. I kept notes on each interview.

Mishler (1986) argues for the respondent to be an active and equal participant in the interview process and that the interview should be an interactional process. Mishler goes on to say that the respondent's perspective and experience, in explicit details, are very important in order for the interview to have greater relevance and that it is important to empower the interviewee to speak in her/his “own voice”. I encouraged each participant to fully describe their experiences and thoughts by being an active listener, by reflecting, and using other cues to further elicit detail and emotive responses. "It is a story that is uniquely the respondent's in that only his or her own voice can articulate it authentically” (Gubrium and Holstein, 2002, p. 19) thus "procedurally, the point is to provide narrative opportunity for this ownership to be expressed, to reveal what presumably lies within” (Gubrium \& Holstein, p. 19). Experience is extremely subjective and in order to gain insight and understanding of the richness of what an individual perceives and feels about a particular set of circumstances that she/he has partaken in, the interview is a highly useful tool.

\section{Protection of Human Subjects}

As a doctoral student at Victoria University of Wellington I obtained authorisation to do this study through the Victoria University Human Ethics Committee. I sought permission to interview six students but revised this to broaden my interview pool to include 10 participants. Permission was granted (see Appendix E).

Following the institutional review process, the identifying of potential informants was initiated. As mentioned previously letters were sent to potential participants and they were asked to respond if they were interested. Prior to any of the interviews informed consent was obtained. The consent form focused on: 1) participant confidentiality 2) custody of tapes/transcriptions 3) voluntary participation 4) withdrawal at any time from the study (see Appendix C). 
Audiotapes and transcriptions have been kept in a locked file cabinet in my office and I have the only key. Both copies of the tapes will be returned to the informants following the acceptance of this thesis. The hard copies of the interviews are also stored in a secured cabinet, and will be kept for six years for possible use in further articles, professional presentations, or further data analysis.

\section{Data Collection}

In RTA data generation comprises the use of multiple sources: the self and the journey to the pinnacle, life changing, epiphany, the use of the research literature, participant observation, deep reflection of vignettes and slides, human interactions, and in the case of this study, the taped interviews of student participants in the international immersion programme. The research questions focused the collection of the data. The questions were:

What impels me to return again and again to work with poor childbearing women in the developing world?

How did international immersion programmes impact the personal and professional lives of student participants?

Can the student experiences help me to understand myself better?

A source of data collection was the interviews of participants from the immersion programme in order to elicit participants' feelings related to their experiences. The opening question, aptly described by Spradley (1979) as the grand tour question was: "Please reflect on what it was like for you going to work and live in a country in the developing world as a student nurse. Tell me about it.” Some participants needed little prompting and continued to talk about the experience and its effect on both their professional and personal life; a few needed probes to rekindle their memories. Utilising a grand tour question enabled participants to discuss their world view about the immersion experience. 
In order to harvest a further yield of information questions such as "Tell me more about that: How did you experience that? How did that make you feel?” were posed to the interviewee. At other times my silence, intent listening, or facial expression garnered more indepth subjective information. Additional questions ensued that followed upon the informant's cues, verbal or non verbal, and/or the particular subject matter under discussion.

\section{Data Generation and Data Analysis}

Data generation and data analysis were an ongoing process consistent with RTA. Tapes were transcribed and carefully read and reread by me in an effort to understand each informant's meaning (see Appendix F). Manifest and latent content analysis were used as I reflected on what I heard during the interviews. The interviewees addressed the immersion programme as affecting their lives both professionally and personally. Issues such as ethnocentricity, culture, prejudice, poverty, living conditions, health care and health care access were frequently talked about from both the immersion experience and their current practice. A constant comparison technique described by Lincoln and Guba (1985) permitted me to identify themes inductively using both informants' words and reflective auto/biographical realities.

During the interviews and afterwards I was constantly connected to RTA as I listened to the students, read and re-read their transcripts, and coded the data. Bracketing was employed in order to allow themes to emerge without egocentric contamination (Gillis \& Jackson, 2002; Holloway \& Wheeler, 1996).

There were two levels of coding completed during early data analysis. Each transcript was read and basic themes were identified. These initial themes included basic concepts about the immersion experience. Level II coding categories were created across transcripts to capture the meaning of the experience to the group rather than individuals. A third process involved my own deeply contemplative process in which I sought answers to the research questions posed. I struggled with both the process and form of the auto/biographical method, at times making notes, at times listening to my beloved Baroque ecclesiastical repertoire for inspiration, 
searching for a way to understand professional activity I have engaged in for many years. To manage the data, I looked for patterns and themes that were later shared with informants to ensure that I had not misrepresented their experience(s). Throughout the process, thick descriptions of the meaning of the immersion programme to informants and subsequently the meaning, to me, emerged (Geertz, 1973). Thick descriptions functioned to "make sense out of expressions of experience” (Denzin, 1989b, p.108).

\section{Rigour}

Qualitative research is generally measured by rigour rather than "reliability" and "validity" commonly employed in quantitative studies. In qualitative research, rigour refers to the trustworthiness readers can put into study findings based on specific criteria/indicators. Miles and Huberman (1994) recognised trustworthiness as present if the following essential elements are met: the research that utilises more than one theoretical approach; indepth knowledge of the subject and setting to be studied is assured; a robust curiosity for theoretical knowledge is present; and the research documents one's ability to carefully explore and scrutinise the matter under study. These elements are also discussed by Guba (1981) as applicability, consistency, neutrality and truth value, each of which I will explain.

Applicability is a particular challenge with qualitative studies conducted in natural settings or with heavy cross cultural contexts. A somewhat better indicator of applicability is “fittingness”, according to Guba (1981). Fittingness means data is fully and richly described, accurate to a fault, and open to the identification of common themes not geographically bound. As I conducted my interviews, I found informants were more likely to describe these common themes such as "embracing visitors" or "celebrating the presence of outsiders" or "being able to non verbally communicate" or "acceptance and flexibility". These themes once identified in one interview could be seen in others. By using constant comparison techniques, back and forth, back and forth, I was able to fully capture what informants were telling me since it is the responsibility of the researcher "...to provide the data base that makes transferability judgements possible on the part of potential appliers” (Lincoln 
\& Guba, 1985, p. 316). I paid attention to describing in detail the immersion programme, the interviews by informants, and interpreting the informants' commentaries and asking for feedback beginning with my earliest analysis. I utilised Moustakas' (1990) heuristic process in order to ensure that I “covered all the bases” by constantly reflecting on the initial engagement, immersion, and incubation components common to RTA (Johnstone, 1999; Moustakas 1990). My cultural socialisation process is also a part of this thesis as well as how it has affected who I am and how I “operationalise” my life.

The second component of rigour in qualitative studies is consistency, or dependability, which means “...data stability over time and over conditions (Polit et al. 2001, p. 315). Krefting (1991) describes consistency as “...whether the findings would be the same if the inquiry were replicated with the same subjects or in a similar context” (1991, p. 216). In qualitative research it is the informant and the researcher “...that are assessed for consistency” (Krefting, 1991, p. 216). The method that I incorporated was the extensive paper trail which showed each and every step and nuance of this study. My focus was to learn all that I could from my informants, represent rich descriptions, and "known variability”. In qualitative research there are multiple realities which “...reflect...that situations constantly change and people’s realities differ” (Gillis \& Jackson, 2002, p. 216). One method of assessing dependability is paying attention to the audit trail whereby the researcher describes, defines and follows a systematic course of study throughout the research project, from inception to conclusion including the written report (Guba, 1981; Gillis \& Jackson, 2002). Consistency has been met by clearly describing the conceptual orientation on which the study was built (RTA, selection of informants, and data management). I thought deeply about every step that was taken in this thesis describing in detail the immersion programme; addressing the cultural socialisation process that I experienced during my formative years; researching the use of auto/biography as a valid qualitative research process; and carefully interviewing students from each of the programmes. All my decisions related to participants, data collection, analysis and conclusions have been included in this report and will be incorporated into the thesis (Sandelowski, 1986). 
Neutrality, or the distance between researcher and subjects, is the third parameter of rigour and refers to what Guba (1981) calls confirmability. This is the neutrality of the data, when creditability of design, of the informants, of the paper trail, of appropriate descriptive data are met. Miles and Huberman (1994) call the concept “objectivity” and Sandelowski (1986) labels it as "having no bias” throughout the research project. Krefting’s (1991) position that "Reflexive analysis is also useful to ensure that the researcher is aware of his or her influence on the data” (p. 221) is an extremely important point because if this is not in place the bias of the researcher may influence all aspects of data collections and results. Guba (1981) informs researchers to ensure that their findings are only those of their informants and the circumstance of the research rather than that of the researcher and her perceptions, ideas and thoughts. "Qualitative researchers “...try to increase the worth of the findings by decreasing the distance between the researcher and the informants, for lengthy periods of observations” (Krefting, 1991, p. 217). This means that in order for the researcher to heighten the significance of the results of her research she must narrow the distance between herself and her informants.

Neutrality was ensured by the data being based on the interviews of the participants of the immersion programmes in order to maintain the rigour of the study. I knew each of the participants of the programme through the theoretical segment of the immersion programme which took place for six months. The participants and I were well known to each other as we lived our immersion experiences together. I also knew each student as part of the nursing programme. I was a woman wearing many hats, as a teacher, resource person, practitioner, nurturer, housemate, support person, programme coordinator and most of all maintaining an excellent rapport with all my students at many emotional levels. I maintained my neutrality by a thorough understanding of the individual and her/his rights to autonomy, the individual's own value concepts, by the individual's uniqueness as related to her/his character and personality and by respecting each participant and her/his thought processes.

The last of the four parameters that ensures rigour is truth value. This, as described by Lincoln \& Guba (1985) reflects the overall credibility of the research 
undertaken. Krefting (1991) states, that this is the very intensity, vibrancy, vividness and fullness of the participants’ subjective descriptions and views. Lincoln \& Guba (1985) used the term prolonged engagement as a strategic approach in order for participants and researcher to become acclimatised and comfortable with each other. Kielhofner (1982, as cited in Krefting, 1991), described the importance of "intense participation, suggesting that it enhances research findings through intimate familiarity and discovery” (p. 217). Both the students and I were constantly immersed in health care delivery, working with local health care providers, and we also lived amongst locals and socialised with them by participating in their family events and other aspects of their daily lives.

Another key component to the credibility of the research is for the participants to be included in the research process by ensuring that the researcher has correctly interpreted their data (Lincoln \& Guba, 1985); “This strategy of revealing research materials to the informants ensures that the researcher has accurately translated the informants’ viewpoints into data” (Krefting, 1991, p. 219). This has been done by keeping in touch with the informants and ensuring that the researcher's perceptions were accurate.

I have carefully ensured credibility by being a very attentive listener, by taking notes, by informants' access to amend their transcripts in order to clarify their salient concepts and having participants identify their themes or patterns used in the thesis in order to ensure accuracy. I am also familiar with the conditions of the developing world, their health care systems, and the many and varied people with whom I have worked, practiced, and lived over a period of many years.

Rigour has been maintained throughout this thesis by the researcher separating her feelings and thoughts from those of the participants in the immersion programmes, by using thick rich descriptions of the participants, by having a very explicit "paper trail”, and by identifying common thematic processes identified by the participants by vigilantly paying attention to the subjects' words, and also by conducting interviews that were guided by the participants. In summary all four aspects of rigour have been met as indicated. 


\section{Summary}

This chapter has discussed the study design, data collection, generation and analysis, sampling process and a review of RTA. The components of rigour, fittingness, consistency, neutrality, and truth value were examined at length. The next chapter will focus on the further unfolding of my story, and perspective. 


\title{
CHAPTER IV
}

\section{INCUBATION: THE REAL STORY}

\author{
"If a [hu]man does not keep pace with his companions, \\ Perhaps it is because he hears a different drummer, \\ Let him step to the music which he hears, \\ However measured or far away" \\ Thoreau (1854/1985, p. 581).
}

\section{Introduction}

The prior chapters have discussed the immersion programme, the theoretical underpinnings of this thesis, and the methodology used. This chapter will address my personal and professional life journey and my family background.

\section{The Real Story}

My personal and professional life journey has taken place over a period of many years and has spanned six of the seven continents. I have followed my heart, in a geographic as well as a psycho/socio/cultural rite of passage, living and working with poor childbearing women throughout the developing world. I have been greatly affected by observing and participating in the child birthing experience of women from all stations and circumstances of life. I have often thought about my impetus to go abroad, and the drive, to return again and again to a world entirely different from my own. I have pondered inordinately and considered the issues of respect and dignity and its role in health care. In 1985 I began to take small groups of nursing students along with me in an effort to broaden their world views. I'd go abroad. I'd help. I'd work. I'd educate students. I'd work with patients, their families, and communities. I'd return home for a year or two then do it again.

What I saw, in most instances, were things like: disorganisation of care, health crises, anger of care providers, lack of health care, lack of resources, low levels of patient and provider education, harshly critical staff members, grief, loss, 
and personal tragedies. Despite these negative occurrences I watched and participated in the most fundamental of human experiences graphically played out during the birthing process again and again and again. I am reminded of a young married girl and her story, typical in the sense of the lack of perceived control, in all facets of her life. , Labour and Delivery/Post Natal

I have recently arrived and my orientation to labour and delivery is taking place today. There is a 16 year old married parturient who has just been walked from the $2^{\text {nd }}$ stage area to the delivery room and has been placed in lithotomy position, She is very shy and embarrassed, and endeavours to hide her face. The fear at what is happening to her body is verbalised by screams of "I'm dying, help me." She is left uncovered and the doctor on duty proceeds to perform the requisite large episiotomy [primigravida]; nobody responds to her verbalisations or her non-verbal communications.

This young woman has been in labour for nine hours, lying flat on her back, with an occasional nurse or doctor stopping in to perform a vaginal examination; she has been the sole occupant of the labour rooms today....alone! No family permitted to visit and no communication with the family representative who is waiting outside. This young woman is from a small village in the nearby mountains and has rarely been outside of its perimeter. Her husband accompanied her to the hospital and left shortly afterwards to return home. Her delivery is not the exception to the rule and the usual fundal pressure, exerted by the nurse standing on a chair, and applying her elbow to the uterine fundus takes place. The patient screams but continues to be ignored.

After delivery of the placenta the doctor does the repair of the episiotomy that take two hours; the client remains uncovered in the lithotomy position with her legs in stirrups. At no time is the client addressed; it is as if she is superfluous, a non-entity, with a perineum through which a neonate has just exited.

Her infant has been placed in a cot in the same room although from where she is lying she cannot see her baby. As yet she does not know the gender but she can hear its cry. She, as a village girl is psycho/socio/culturally out of her realm, and does not ask questions; she is a stranger in this unknown and formidable government institution. She is intimidated by her surroundings, and although trying to withdraw into herself psychologically she is constantly 
reminded of her surroundings and vulnerability by her labour contractions, delivery, and episiotomy repair.

She is discharged to the family friend 24 hours later and will remain with her for a few days until her husband can return to fetch her and the baby. Two days later there is a knock on the door of the house where I am staying; my whereabouts are always known by the people of this small community for I am the "stranger", the foreign nurse/midwife who is always walking about the town getting to know everybody.

The request is for me to come and see the young mother as she is having pain "from where the baby came out". I return with the family friend to her home and talk with the mother who soon starts to cry. I ask her permission to look "below" in order to understand what is happening to her. Upon examination I observe redness, oedema, and ecchymosis. I ask permission to touch and examine her "private areas". She shakes her head yes, and immediately covers her face with a small rag that she is clutching.

The requisite items that I bring with me to the developing world are alcohol swabs and sterile and clean gloves. I use the alcohol to clean my hands and don a pair of sterile gloves. Upon closer examination and inspection and palpation of the vaginal vault and perineum and anal area I do not find signs of an infection; there is no untoward discharge, and there are a few sutures that are not well approximated. The parturient has been using the outhouse, as all of us do, and one must sustain a slight shearing effect as one places oneself in position to void and/or defecate. The seat is made of rough cement and stones that protrude in varying degrees.

I ensure that the mother understands things that she can do to alleviate her distress; simple things that will cost no money and tell her that I will be back later this evening to check in and see how she feels.

As I am leaving the house the family friend accompanies me and as we are walking toward my lodgings she reveals the real reason for the visit. She has gotten word that the husband will come to take his wife and baby home the next day; she fears for the young woman as she knows that the husband will want to exercise his "conjugal rights". She will not be able to refuse. She wants to know what can be done to keep the mother here with her at least for another week.

For me the scenario rarely changes only the characters (players) and geographic location. Women and their babies: labour and delivery, a culmination of the life that millions of women of poverty in the developing world live. 
Powerlessness, pawns, caught up in societies that view them as a means to an end: the creation of new life over and over again. Women, as drudges expected to maintain their households, children, in-laws, and reproduce, and produce; an endless cycle. To be sexually available at all times to their husbands, and when overtaken by death related to pregnancy the husband's role to find a new wife and have a new family as quickly as possible. Sometimes I would like to scream: "Look, see, hear; that person on the delivery table is a human being just like you. She has a family, she experiences the same emotions as you, why is she being treated as if she a nonentity?”

I wonder if the doctors, midwives and nurses pilot on automatic, subconsciously, in order to spare themselves the very real pain, physiologically and psychosocially that they would have to understand and share, the lived lives of their patients. Is this the easier way out? I socialise with the care providers and the potential patients as I become known in the communities; both groups are as humans the world over: caring, concerned, giving. Who am I? How to break this endless repetitious cycle for childbearing women of poverty in the developing world? How would hospital personnel in a medical centre in the developed world respond to suggestions from an outsider?

Am I the ultimate romantic, the ultimate humanist who continues to believe in the potential of each human being. I am in my seventh decade of life and I still hold this belief though at times I question myself when I look at the turbulence and extant tumult of the world today. Planned health change comes slowly from the trust that is developed between care-giver and recipient and I have made changes: small, substantial changes in many of the small communities where I have practiced nursing and midwifery. I have seen changes in the communication styles of personnel, it can happen. It is not a miracle, but an understanding.

\section{Development of Interest: My Story}

One doesn't just “fall” into international nursing. I have always travelled off the beaten path. The most significant part of my journeys has always been the local people(s) and their daily lives, a constant source of fascination and allure. My 
interest in people and learning about them, my caring ethic, my nursing and midwifery skills have been a "package" that has enabled mutual growth and expansion of world views for "local” populations as well as myself. My life has been significantly enhanced and changed by continually building upon my cognitive knowledge through immersing myself into the health care system and culture of the community where I am volunteering. Learning about the uniqueness of each human being, their families and communities and being able to fully participate as a health care provider are a few of the reasons that have propelled me in my pursuit of understanding the world and the unique people(s) that inhabit the earth.

My broad interest in nursing has also served me well. Early in my career I was the nursing supervisor of a busy paediatric emergency services unit at a public hospital in New York City which provided health services to many poor communities. At that time I had more than eleven years of nursing experience in diverse settings and specialties and worked with a wide array of people of all hues and colours. I worked with patients who spoke many different languages and belonged to a plethora of ethnicities. I knew in 1971 that it was finally the appropriate time for me to begin my voluntary service as a health care provider in the developing world.

The unit was constantly bustling and our patients ranged from the chronic asthmatic to severe trauma, to overt child abuse. One evening the Protestant chaplain was visiting and I approached him concerning my proposed plans to work as a nurse volunteer. We talked for a while and I sensed that there was something that he was not able to express. At last, he asked the question about my church affiliation. My answer was that I was not a Protestant. His words were harsh and ominous: "We don't want you; you aren’t a member of a Protestant church”, I was astounded, I was hurt. His comments made me reflect deeply on the role and functions of health professionals who choose to work in the developing world. Was it only one's religious beliefs that afforded one the ability to work well with people, could it not also be the practice of one's profession and the promotion of health that motivated one to global geographic endeavours. I wondered, too, could one not just be a human being who cared deeply about people, their lifeways, and the state of their health? 
A few months later I came in contact with the (Irish) Roman Catholic chaplain and wrestled with these thoughts again. I revised my verbal technique by explaining what I wanted to do and ending with the statement that I was not a Catholic. His response was, "great, that's not a problem”, and so began a long and fruitful association with many wonderful nuns and priests in many countries in the developing world. I learned that human caring was the essence of my interest.

I have practiced nursing and midwifery in many countries and for the last twenty three years I have been a professor of nursing/childbearing health. I began my voluntary professional services in sub Saharan Africa, and have subsequently branched out to include South East Asia, China, the Caribbean, Central and South America, and Eastern Europe. I have also worked in diverse health care settings in both the United States and Great Britain.

This thesis is about my life's professional and personal journey to the developing world and the life changing experiences for student nurses who participated in the Humboldt State University international immersion programmes. As I think of my initial engagement in the developing world I think of an event that took place on my “maiden voyage” in 1971. The following vignette describes my deep feelings which have remained with me all these many years later.

Opisthotonos in [name of country]

The homemade wooden cart drawn by one ox arrives at the courtyard of the hospital early one morning just before dawn. As my quarters are situated at the other end of the compound I am not privy to the commotion that has ensued due to the neonate that has just arrived. He is acutely ill and very distressed. He was born seven days ago and the parents consulted a shaman because the baby's behaviours were different than any of their prior babies. They followed his instructions and after 48 hours decided that the baby's condition was rapidly deteriorating and decided to access medical care at the closest hospital. The parents describe the baby's condition to hospital personnel: the baby was unable to suckle at the breast, he was very hot, the umbilical area was very hard, and his position was "strange". We were a ten hour oxcart journey from their home on earthen rutted roads.

As the story unfolded the parents stated a day and a half after the baby's birth his umbilical cord stump was very red and that the local 
village woman who had delivered the baby suggested a visit to the shaman. When they consulted with him he put soil on the remnants of the cord stump and asked the parents to return with the baby in two days. This was their first son; they had three young daughters.

When a few hours later I arrived for duty I was given report and was taken to the small darkened side room that has been allotted to the parents and baby. He was in full opisthotonus. He was made as comfortable as possible and was receiving parenteral phenobarbital every four hours. The parents sat and watched. They knew that the prognosis was poor. I was overtaken with grief as I felt impotent merely sitting by and watching the baby succumb. I watched an agonizing transition from life to death. The baby spent less than a week in the world.

As I reflect upon this, I say to myself: This happened in 1971. It is now 2006 and I vividly remember this event as if it were yesterday; the pain feels as real today as it did 35 years ago. It makes me feel impotent as a human being. I think about my fellow human beings in non-industrialised nations and also those of us in the lands of plenty, how and where are we positioned? Perhaps the only way to make peace with these differences it to be open to the power of the experiences. I feel that power, it is overwhelming, yet I cannot reconcile myself to the constant loss.

My initial engagement has had many, many encores and I never realised how these multiple "acts” would forever change who I am and how I respond. Moustakas \& Perry (1973) label any human response as “...an expression of one’s own inner experience, a direct expression of feeling...” (p. 7). Because human responses are both learned and emotive, they result in personal development. Self-development through knowledge, learning and understanding, are thus responses to one's lived life, one's emotional self, and one's self expression. True learning for me has come through a contemplative reflective process which is a somewhat unusual qualitative methodology (Barnes, 1981, Boud, Keogh \& Walker, Eds., 1985; Johns \& McCormick, 1998; Morse, 1997; Schön, 1983, Street, 1990, 1992, 1995; Taylor, 2000). Moustakas and Perry (1973) labelled it “...the internal search to discover, with an encompassing puzzlement, a passionate desire to know, a devotion and commitment to pursue a question that is strongly connected to one's own identity and selfhood” (p. 40). I have lived my life qualitatively. I have sought knowledge around the world and at home, watching, participating and considering the lives of 
childbearing women of poverty because "one of the best ways to learn about cultural groups (others) is to observe and interact (participate) with them as they go about their daily lives” (DeSantis, 1999, p. 110).

The multiple issues that I have encountered are much greater than all the pieces of the puzzle parts I have seen. As I approach the last three years of my seventh decade of life, I feel increased pressure from within to describe my passion for life and elucidate and define my feelings and refine my learning.

These issues have totally consumed me for many years as an inner search. I have also been greatly affected by magazine and newspaper articles, as well as the research articles relating to the poverty of families with young children in the developing world, the inability of the developed world and of the multiple individually affected countries to create large scale sustainable planned health and life change for women of poverty, of the morbidity and mortality of the under fives, of the individual struggles of women and young children. I have directly experienced multiple immersion experiences into developing world institutions, and have had endless conversations and explorations with people who live at the opposite ends of a very long continuum, those of the developed world and those of the developing world.

During a period of sabbatical in New Zealand where I was privileged to observe doctoral students presenting their qualitative and often narrative research endeavours for academic critique, I was inspired to write, to question and to reflect on my own story. I felt a compelling need to research and to reveal the meanings of my story through the formal means of a doctoral thesis. A search of the literature on the implicit and explicit dictums of writing a thesis stated strict rules and regulations regarding its format, content and presentation, and also the thorough investigation of a subject that adds to the body of knowledge in a given field (Brause, 2000; Davis \& Parker, 1997; Evans, 1996; Gash, 2000; Hamilton, 1990; Holt, 1998; Phillips \& Pugh, 1998; Roundtree \& Lang, 1996; Turabian, 1987; Watson, 1987). One resource on theses even suggested that it is usually a beginning or mid-career undertaking, emphasising that it is usually the former (Brause, 2000). This thesis however 
diverges from the above as it is a culmination of a professional career in nursing and midwifery, and a personal odyssey that spans forty-five years.

Writing has always been extraordinarily difficult for me, and yet in emotive writing, there has always been something that flowed. I have consistently been able to compose words, full of meaning, when sending anniversary greetings, condolences, or other events marking life's many personal and significant milestones. What was it that enabled me to do that? Could I truly expand my field of "expertise” writing a thesis with much thought and feeling through carefully constructed sentences?

Reflecting on this form of writing brings me to my basic philosophy of life. I am a woman who has spent her life traveling and immersing herself in ways of life so very different than her own. The customs and values of people of innumerable countries have always presented me with an incredible array of belief systems. This has been a major stimulus for me to reflect on the similarities in differences of people and their cultures; I am enthralled by people, their stories, lives, ways, and families. I have learned and grown so much due to people's generosity in opening up and sharing their lives with me.

I have also been fortunate to be able to practice both nursing and midwifery as a member of the health care team of many communities in the developing world as well as working with diverse populations in Great Britain and the United States of America.

I was raised in an extended family that had as one of its basic tenets, the belief that all human beings are unique, important, and that every person deserves to be treated with dignity and respect. I think of my youth and my parents and their thankfulness of being born in a country where opportunity ostensibly existed for all and where one could live without being impacted by prejudice.

My parents were the children of Jewish immigrants escaping Russian racism of the late 19th century. In Europe, for generations, my antecedents lived in dire poverty where education was only for the privileged. Their escape to the "land of the free" was fortuitous as our family unit would never have survived the Stalin and Hitler eras. My grandmothers were illiterate women who worked in the garment 
industry of the early 1900’s in New York City. My grandfathers held menial jobs throughout their lives. My parents did not graduate secondary school as it was necessary for them to help ensure that the extended family would survive economically.

I live in a nation that considers itself multicultural, but with the passing of the years, I realise that this, indeed, is not so. Where, is the participation in government, in business, in the accustomed mainstream social life, of those that “differ” Where is the "visualness” of a people who are dissimilar, "act” differently, and have features and body types that do not fit into the mould of the majority? When I was growing up I was part of that “differentness”.

People, their lived lives, their ways, their celebrations, my extended family upbringing; me, always wanting to learn about how other people live and what is meaningful to them, constitutes my ever growing lexicon of world ways and the knowledge of the similarities in differences of human beings.

Perhaps it is my "eticness" and my belief in people that has been the attractor in my endeavours to understand other "others" who have not been part of the mainstream culture. Perhaps it is my lived life. I look back and remember my father, who had a small business, and always employed ethnic minority peoples in order to give them a chance to get started in life. I remember bringing home friends, in both primary and secondary school, who were not members of mainstream society, and each was treated as a person with individual needs, aspirations and just being a buddy of their daughter....no matter what the colour, shape, or belief system.

I also remember myself as a youngster always concerned and worried about others as I believed, and still do, that we are not individuals afloat on a planet, but interwoven and interlocked into a greater schematic. My inner voice (Douglas \& Moustakas, 1985; Moustakas 1956; 1990; Polanyi, 1962) and I have always conversed, thought about, and agonised over human inequities.

Marginalised people are everywhere and where are the opportunities for them to better their lives and be active members of the institutions of the majority culture, have access to formal education, and most importantly, access to health care that is financially and culturally relevant? Societies, the world round, appear to be 
focused on the status quo, on the maintenance of that which is. "Lip service" is given to the problems of those who are not in a position of power.

When I reflect on the powerlessness of the powerless I think perhaps it is that I am the ultimate romantic. I am the product of my socialisation process, as is the other, and it still deeply affects my actions in both my professional and personal life. If one is powerless and does not have perceived control, is it justifiable to perpetrate violence upon them, especially during the vulnerable time of childbirth that is compounded by the anxiety and fear of the labouring woman (d'Oliveira, Diniz, \& Schraiber, 2002). A woman with no education, a village woman, a woman alone, relatives dispersed by the staff, a poor woman, who is a "foreigner" in a formal institution in her own country: should she be the victim of cultural biases which then gives permission to other forms of accepted behaviours, simply based on her station in life?

I spend considerable time wondering about, I, as the standard of a belief system, the erosion of the family unit, in whatever forms it takes and the uni-focus of consumption and materialism, that contribute to an altered sense of community. With such tunnel vision can there be a world wide concern for others and the quality of their lives?

\section{Immersion}

I am a woman who has melded her professional and personal life to focus on poor childbearing women in the developing world by committing herself to actively practising as a midwife in poor communities. I felt that I must write and cry out to the world of the privileged, LOOK, LISTEN, FEEL: don't you know what is taking place everyday to millions of poor childbearing women in the developing world? I felt it as one of my missions in life, to get the word out, and so embarked on a journey of presentations to countless audiences in myriad countries. I now felt compelled by an inner need to write about my many experiences of work and living in the developing world (Polit et al. 2001).

I recently heard a very apt metaphor on National Public Radio in the United States (Toms \& Toms, 2003). It related to a problem that a tree was undergoing: so 
the leaves were trimmed, and as that didn't work, a branch was cut off, the problem continued, and another branch had to go. Finally the storyteller said: in order to solve a problem "look to the roots", and I echo that palliative treatment in maternal/child health treats symptoms but never gets to the root causes.

After many years I realise just how impotent I am. Who am I to enter another culture, and propose change(s)? “...no culture can be judged from outside and no individual can challenge the moral worldview of another” (Leo, 2002, p.14). Organisations, such as Family Care International, and the United Nations have been working toward decreasing the number of maternal, neonatal, and infant deaths in developing nations yet, target dates have consistently been moved in order to accommodate what has not taken place.

From my earliest memories I have always marched to a different drummer than most people. I have always felt the pain of others whether human or animal, for we are our “brothers' keepers”. From this philosophy comes this work.

\section{Summary}

This chapter has discussed my personal background and perspective and my family of origin. The next chapter will include the participants’ biographies and the findings of the study. 


\section{CHAPTER V}

\section{FINDINGS PART I: ILLUMINATION AND EXPLICATION}

“...we [as nursing educators] have maintained a passion for molding and shaping and teaching and opening the eyes of individuals who, upon graduation, join us as colleagues in our chosen profession"

(Accord, 2000, p. 128).

\section{Introduction}

This chapter will discuss the findings of the study. Ten interviews were conducted with students who had participated in the immersion programme. Each interview began with a grand tour question (Spradley, 1979) and was followed by probes in order to stimulate and arouse each individual's memories. The aim of the interviews was to answer the research questions by deepening my understanding, through information retrieval, of my reasons for developing and continuing an international immersion programme. Understanding can be thought of as the definitive purpose of research (Munhall \& Oiler, 1986), when the unknown is revealed and is also evocative (Spinelli, 1989). Reflective topical autobiography (RTA) was the methodology utilised and Moustakas' (1970) heuristic research model was employed in order to adequately answer the research questions posed in this thesis.

The research questions were:

What impels me to return again and again to work with poor childbearing women in the developing world?

How did international immersion programmes impact the personal and professional lives of student participants?

Can the student experiences help me to understand myself better? 


\section{Participant Biographies}

Ten nurses were invited and agreed to participate in a semi-structured interview process. All ten had participated in a summer international immersion programme that lasted between seven to nine weeks, while enrolled in a baccalaureate nursing programme in Northern California. The following section will provide a brief description of each participant to indicate the diversity in age, marital status, parenthood status and gender. The stage at which participation in the immersion programme occurred and some details of professional employment history and future plans since participation in the programme will be included. Pseudonyms were chosen by each participant to disguise their identity which remains confidential to the researcher.

\section{Alice}

Alice participated in the immersion programme in the year 2000. She had just completed her Bachelor of Science in Nursing, was married, and was 31 years of age. For the first two years following graduation Alice worked on a medicalsurgical unit of a small rural hospital. She is currently working in the same hospital on the obstetrical unit. This includes ante natal, labour and delivery, post natal and the newborn nursery. She has plans to attend a graduate midwifery programme and become a Certified Nurse Midwife with a Master's degree.

\section{Bugs}

Bugs had completed her first year in the nursing programme when she participated in the immersion programme. She was single and 23 years of age. She has been working as an infection control nurse as well as a critical care nurse in a small rural hospital. Initially she worked on a medical-surgical unit. Her future plans include two certifications, critical care nurse (CCRN) and infection control nurse specialist (CIC). Bugs is planning to do a Master’s degree in Public Health. 


\section{Gigi}

Gigi participated in 1996, the year she graduated from the nursing programme. She was married for two weeks at the time of embarkation and was 27 years of age. Currently she is the mother of two small children. Her professional work history includes her continuous work in the ICU (Intensive Care Unit) in a small rural hospital. She has been a charge nurse for the past five years. She is an ACLS (Advanced Cardiac Life Support) instructor, and an associate faculty member at a local junior college that offers an associate degree programme in nursing. She will be starting her Master's Degree in nursing in six months doing an on-line programme.

\section{Jasmine}

Jasmine had completed her second year in the nursing programme when she participated in the immersion programme. She was 21 years of age and single. Jasmine's work history includes a year of medical surgical and neurological nursing in Alaska working with many native peoples. She has also worked in a skilled nursing facility with an elderly population. She recently worked as an $\mathrm{RN}$ at a resident summer camp. She will soon begin employment as a school nurse in a rural secondary school. She is a certified massage therapist. Jasmine's professional plans are to do community nursing but she is uncertain at this time as to exactly what she envisions her role will be.

\section{Joan}

At the time of the immersion programme Joan had completed her first year in the nursing programme and was 21 years of age and single. Since graduation she has been working in a busy maternity unit in a large metropolitan area. Her specialities are labour and delivery, post natal, and high risk perinatal care. She also works at a free-standing birth centre in a large metropolitan city. Joan plans to attend midwifery school and practice as an independent midwife. She also wants to work as a midwife in the developing world. 


\section{Kaitlyn}

Kaitlyn participated in the programme in 1991 at the end of her second year in the nursing major; she was 35 years of age. She was single at the time and the mother of a nine-year old son. Since graduation she has been employed locally as an ICU nurse, worked on a medical-surgical unit, developed a quality management programme for physicians, and was the RN administrator for a non-profit mobile medical unit. She is currently not employed as an $\mathrm{RN}$ due to health reasons.

\section{Lee}

Lee had completed two years of the nursing programme at the time he participated in the immersion programme, 1997. He was 46 years of age, married with two sons. Lee was a veteran at the time he entered the nursing programme. His nursing career has included work on a medical-surgical unit, and as a travelling nurse. He is currently employed as nurse in a state penitentiary. At the present Lee has no plans to return to school.

\section{Natividad}

Natividad participated in the programme in 2000 and had completed one year of the nursing programme. She was 20 years of age and single. She has been working as a staff nurse on a cardiac unit in a rural hospital as well as being a quality assurance director for a home health care agency where she helped to develop "best practices" for the agency. She is a certified holistic nurse with the American Holistic Nurses Association. She has been accepted to begin her Master's degree/ $\mathrm{PhD}$ in acute care nursing in a year's time. Natividad has been a co-author, with three published articles in refeered journals and has presented at national conferences in the United States.

\section{Nicole}

Nicole participated in the immersion programme at the completion of her first year of nursing studies, 1997. She was 29 years of age and married. She has worked as a medical-surgical nurse, and on a cardiac telemetry unit. Currently she is 
working on a medical-surgical unit. She has two children and at the present does not have further educational plans.

\section{Trae}

Trae participated in the immersion programme in 1996 at the end of her first year in the nursing programme. She was 43 years of age, single, and the mother of a 12 year old son. Her work history includes medical-surgical nursing, obstetrical nursing, intensive care, staff development co-ordinator, certified CPR instructor, certified First-Aid instructor and she is currently employed in home health care and staff development. She is working in a rural setting and has her BLS (Basic Life Support) and ACLS (Advanced Cardiac Life Support) certification as instructor and trainer. At this time Trae is working for a local community agency as a home health nurse and staff developer. She is working toward her Master's degree in acute care nursing.

\section{Findings}

An in depth interview was conducted with every participant. I asked each one to reflect on their experience and to describe what they remembered and what impact it has had, if any, on their professional and/or personal lives. Interviews lasted 60-95 minutes and each was fully transcribed and then analysed as described in Chapter III. This section provides the details of that analysis using excerpts from all participant transcripts.

The analysis of the transcripts revealed that there were multiple common themes that arose from the data which were then placed into three major categories representing the themes. Each category will be discussed which reflects the events, feelings, meanings and participant interpretations of their immersion experience. The three common categories are Having Blind Trust, Valuing Others, and Transforming Experiences. Each will be explained more fully. 


\section{Having Blind Trust}

The first category developed from the data analysis, or revealed through the process of data analysis I named "Having Blind Trust". Trust is defined as having confidence in, relying upon, or depending upon (Flexner, 1983). In this study, trust was established individually and collectively with hospital personnel, as well as in social situations. Blind trust means acceptance based on face value, on things being as they are. "Having Blind Trust” inferred acceptance with an absence of thought, decision making or processing. Immersion programme participants talked about "Having Blind Trust” in themes that included "Welcome/acceptance”, "Building rapport”, engaging in “Teaching/learning”, establishing “Open relationship with patient care”, and developing “Camaraderie and rapport”.

The "Welcome/acceptance" that students received at each site by their nursing peers, nursing teachers, community members, and health care workers was cited as amazing. They were blindly accepted in the host countries as evidenced by official receptions, parties, press conferences, get-togethers, dinners and invitations to personal and social activities with both the local populace and hospital personnel.

Table 5.1

Major Themes and Categories

\begin{tabular}{|l|l|}
\hline Themes & Category \\
\hline Welcome/acceptance & Having Blind Trust \\
Building rapport & \\
Teaching/learning & \\
Open relationship with patient care & \\
Camaraderie and rapport & \\
\hline Beyond language & Valuing Others \\
Being a human being & \\
Caring about and for others & \\
Sharing lives & \\
\hline Taking risks & Transforming Experiences \\
Assuming advocacy roles & \\
Recognising prejudice & \\
Having life changes & \\
\hline
\end{tabular}


Participants were very excited by this acceptance, feeling that they immersed themselves quickly into the cultural environment in which they found themselves. According to the participants, being able to savour the daily nuances of others' everyday lives and work alongside health professionals whose roles and functions differed was an exceptional experiential way of being welcomed and accepted.

"Welcome/acceptance” was portrayed by Gigi as:

I was relieved at the welcoming, the people were just so excited to see us and so grateful that we'd come and it was really exciting for them and for us to meet them finally. When talking about the welcoming I have several vivid memories of doing things with the students and/or instructors either at the school or their family homes.

Their families cooking these meals of wonderful food and drinks and things that were really the kind of food that those families commonly ate; that was really exciting.

I remember this one family, the mother was so warm, she was just hugging us and making us so welcome and we had this wonderful meal and that was really fun. I remember, the students and the faculty having welcoming kind of parties, get "togethers" for us and there was all this singing and dancing and music and games and things that we would never really do here. I just can't imagine it, everybody would be sitting there and having a good time. It was really fun because it was genuine. I have this total memory of a party, it was a big party and we did a little skit where we wore big nursing hats and we were dying laughing. We thought it was hilarious; we were asked to participate in this performance and it is nothing that we would ever do and just going, oh my goodness, what are we doing. But it was really fun and they really went all out for us.

Natividad talked about a get together that happened within a few days of our arrival. It was in honour of her birthday and reinforced her beliefs and visions of the outpouring of feelings of welcome and acceptance.

To have such genuine good wishes and such a warm gathering with amazing people who had only known me for about 48 hours just floored me. Not just because of the personal significance, but because this celebration took stereotypes and hardship and distrust and reduced it to the essence of love and humanity.

Lee discussed "Welcome/acceptance” that included an element of safety.

I was really nervous going in because we were not far from a war zone. In the unit we lived, we at all times felt safe and accepted. We 
walked three kilometres to the hospital every day. To know that people accepted you and constantly offered to help really added to our comfort levels as everybody was extremely friendly and kind. One day I was out walking and all of a sudden a car pulls up next to me and a man jumps out. I'm thinking, well, what is going to happen next. And the first thing he said was, "are you Lee from America?” I said yep! He said his wife works at the hospital and he was to take me anywhere that I wanted. We ended up on a short sightseeing tour. Everybody knew who we were!

For Alice safety was also a large part of "Welcome/acceptance"

Both, in my personal and professional life, I often find myself talking with people who have travelled or are foreigners. I talk about my journey to and that I worked there as nurse; it really makes people feel more comfortable and makes them really want to open up because I've taken that step. I've left my country, my safety zone, I've been somewhere else and I can relate to their experience and they feel safe opening up to me.

This makes me feel more confident and excited to continue working with people who have different value systems than I do. It makes me feel like I've opened up part of myself that I thought was open before. I've always said that I'm really interested in people. I'm interested in where they're coming from but really, I'd never stepped outside that safety zone.

Alice further described how different, yet liberating, the trip was:

I considered myself a person who is knowledgeable about international politics and how they affect people. I thought I was very sensitive to that matter, but now I feel much more sensitised because I realize these are my friends they're talking about on the news, that are suffering for lack of healthcare, poor sanitation, lack of potable water, basic necessities, and living in cardboard shacks.

"Welcome/acceptance" was described by Trae as being like royalty; nothing was too special or too much work. Only the best would do:

What comes to my mind is something that I didn't recognize until we had been there a couple of weeks. I realized that we were being treated like, like royalty, like honoured emissaries from another land. We were treated incredibly well. On the many days that we travelled to the outlying communities the people in the villages would dress up in their Sunday best. I think it was on the $10^{\text {th }}$ or 11 th community "talk" that it dawned on me that the folks didn't always walk around with shoes on. The girls wore freshly laundered prim pinafores, and the babies were in colourful homemade outfits. The boys were dressed in their finest clothes, too. The whole village 
would come, teenage boys and girls, elderly grandfathers and grandmothers, and you know, dumb me, it was just like it dawned on me, oh my, this display of finery and kindness is for us. That really impacted me. The people treated us so courteously.

The second theme contributing to the category of "Having Blind Trust” was "Building rapport" Nicole described taking the time to sit and wait for a client to trust her. She was able to establish rapport with her client by engaging in the simplest of human behaviours:

I was making rounds with the nurse and giving medications and I met one patient, who was in a five bedded room, which was a new concept to me. The healthier you got the greater number of patients there were per room. This man spoke a little English and he was very nervous about me giving him his injection and I don't know if it was because I was not a ___ [name of country] nurse or if he was just nervous about the shot. I had to gain his trust and I spoke very slowly and clearly. I'd never sat down on a patient's bed but this man moved over and wanted me to sit and I remember just talking to him for about three minutes and he then felt comfortable to depend on me to administer the medication. He explained that he was in hospital due to an accident on his farm. Just sitting and talking with him and telling him something about myself and my purpose until he trusted me to carry out my nursing functions and was satisfied that I knew what I was doing was very important. We take for granted that there is immediate trust and I really had to gain this patient's trust. I also used touch as communication in this instance

Lee talked about developing rapport in interpersonal social situations. For example, regularly playing basketball with the young boys, teenagers, and the dads of the town easily connected him to them.

I played a lot with them and that's where I really made friends and got to be known by the people and gained their respect. It was a great experience. Everybody also wanted to practice their English skills so we would sit around and chat and this continued to build trust between myself, the other students and the local populace.

The idea of "Building rapport" was also described by individuals as they worked one on one with a local nursing instructor. Joan talked about it this way:

Many people who worked in hospital invited us to their homes, which was so interesting because that's not something that is customary here [USA] to just meet people at work and invite them to meet your family. One of the experiences involved a woman who worked in the hospital cafeteria; it was really wonderful. She was 
hilarious, she just made us laugh so hard. They had the whole family there. Her 16 year old daughter spoke English and was our cultural and linguistic interpreter. It just went on and on and just back and forth.

The third theme contributing to the category "Having Blind Trust" was engaging in mutual "Teaching/ learning”. This was seen as a "Having Blind Trust” issue in that each party was open to discussing and looking at issues and problems from completely different mind-sets. This served to deepen their understanding of one another. Informants talked about the excitement of learning new ways of doing things. They also expressed feelings of anxiety that accompanied the excitement due to being nursing students and not feeling very experienced. They found that experiential learning was a growth experience and felt increasing confidence and comfort in learning new ways and teaching about their own way of doing things. They were open to our seeing everything: "the good and the bad".

For example Alice said:

They [nurses, midwives] were just astonished that we wanted to be there and work with them. For the nurses to have a group of American [USA] student nurses coming to learn from them was a surprise, in fact, it was a source of continuing amazement for the nurses. "Often we host [Alice] foreigners and when they come to visit we show them the pretty things and guide them to see what we want them to see. They were very open and showed us things that our institutions would not share with a foreigner; the good, the bad and the ugly.

Nicole spoke of the learning process as trust based and reciprocal.

We really forged trusting relationships with the nurses; they taught us a lot and we taught them a lot too; it was a reciprocal process. We really bonded with the nurses.

For Bugs learning how to do a dressing change without gloves and with minimal equipment was a unique "blind trust” learning experience. She was shocked at first and then shared with the staff the way she had been taught to do dressing changes. This was a learning experience for all involved, for the local instructor and student nurses had never experienced a dressing change where the care provider directly touched the wound site wearing gloves. For Bugs, using forceps to do the dressing change was a totally different way from how she had been taught. Bugs 
described her fears, as a challenge, and described the learning process in the following way.

Why are you going to wear gloves? Why don't you just do it the way we do it, we don't wear gloves and you aren't going to touch the wound with your bare hands; don't worry about that. I thought at first about just running out of the hospital. Then I thought okay, I'll do it the way they do it and so I did using a haemostat and forceps and it was actually fun. I learned all about taking a small cotton ball and tearing it into four little pieces because it was so expensive to purchase.

The instructor also said that it was the monsoon season and the cotton supplies come from ___ and if the truck cannot make it because of a washout we will then not have any cotton balls. I was kind of stunned with that information; I had no idea. Afterwards she said to me you know you come from a land of waste. At first I felt like I had been slapped in the face, land of waste! And then realised, thinking back on different things I would do in the hospital and things that I had seen, that she was right.

When I came back home and went to work the very first day all her words were just ringing in my ears because there it was, all the dressing change supplies, boxes and boxes of things in each patient's room, most never opened. Then when the patient is discharged and does not wish to take the supplies home we throw it all away because it's been in the patient's room and we can't reuse it on another patient even though the box is sealed. And I thought well she's absolutely right. I guess I do come from the land of waste.

This was clear evidence of "Having Blind Trust” as described by Bugs.

Initially Bugs panicked but quickly regained her composure and was open to learning an alternate method of a dressing change.

The students said that the learning experiences opened them up to being more flexible and adaptable and that the fear related to learning new ways decreased with their continuing exposure to new experiences. Jasmine described it like this:

It's something that happens with experience and I'm really inexperienced as far as the world and careers go. It's an ongoing learning process and I'm still learning to put the pieces of the puzzle together. If you can just let go and understand that you don't have to do everything in a linear way. You gradually become more comfortable with being creative and innovative and realise that the sum is greater than its parts. I'm still in that process as I've been bopping around in different nursing specialities. It's always the new 
learning experience that helps and each time it gets easier to be flexible.

Establishing an open relationship with patient care and recognising the importance of each person as a unique individual embodied "Having Blind Trust" as well. Kaitlyn talked about the experience of working in the developing world as being a "humbling one".

It was humbling to practice my nursing skills learned in the United States. Without resources I realised that all I could sometimes do was the best that I could do, which was often simply to be as compassionate as I could.

For Natividad a comatose and dying paediatric patient, four years of age, allowed her to experience a truly open relationship with another human being. The child was moribund from the very first time that she worked with him, and he succumbed to his injuries after a few weeks.

That was the first time I'd ever listened, smelled, and felt death and then the knowledge that I was in the presence of a child who was dying so wrongly. I had this role where I was there for him and holding his hand and by his side with the other two students that were with me. I was there as a nurse and to be there in that role and see this process with a four year old was probably one of the most heart wrenching things I'd ever done. But yet I found such peace because I knew that I was genuinely there for him - not there to push medicines, or put tubes or needles into his body, but to just be there with and for him.

For Joan, having an "Open relationship with patient care” meant being more flexible and understanding.

I have found that working in another culture and learning the things that I did has made me more flexible and understanding. Each patient has a unique set of needs. Being able to not be judgemental and just work within the environment of what your patient wants, by having an open relationship simply works in one's favour. Being together with a patient was, at times, as simple as doing whatever the patient said.

The fifth and last theme, in the major category of "Having Blind Trust" was “Camaraderie and rapport”. Companionship, closeness, familiarity are words that that the students used to describe this phenomenon. "Having Blind Trust" happened 
both in the workplace and in social situations. Trae talked about connecting as heart expanding:

Frankly, it's a big deal to fly to another country and get there and be immersed in another culture and another language. To live with a family, to totally eat their food, be part of their daily lives, wake with them, sleep with them, hear the same noises they hear at night, walk the streets of the town and say hello to people and feel totally safe and be a respected part of their community. The connection to those you meet helps you grow as a person and expands your heart; it made me more compassionate and caring. It's a much bigger experience than just work; it makes you understand how you can become part of a community and make a difference too.

Alice captured connectedness as she described nurses the world over united by their profession and a feeling of camaraderie. She described it as a big family:

It is hard to explain, but the experience of being in makes me feel like I belong to a big family around the world. They way they treated us and brought us into their homes and shared everything with us just because we were nurses from another country and they wanted to share their lives with us. It makes me want to share mine with whomever I touch.

When you travel around the world and you meet somebody who's a nurse, there's this immediate understanding and bond that exists, a foundation to begin a relationship. Alice said: “Our experiences with the nurses were great; I would like to have done more to thank them”.

Nicole described camaraderie and rapport as:

Young children are great to play with and they don't care whether you speak their language. They're going to like you or not like you. The refugees letting us play with their children and not knowing us and smiling at us trying to say hello. The feeling of closeness that developed between the children and us as well as the parents and our group was amazing.

Jasmine was very taken by the camaraderie of the nurses and felt that it was something that has been lost in the world that she lives in. She felt that technology was one of the causative influencing factors.

What I really learned about over there was the existence of the camaraderie amongst the nurses. They were all friends and always helping one another. I remember just being with them, holding the gauze, those little triangles that would then be autoclaved. They were 
all sitting in the staff lounge working on that together and talking about their families, and including us in their conversations. Here we have the technology and we are also given many more duties and responsibilities. Here we're very focused on time, i.e. medicines, assessments and treatments. The time for bonding with our coworkers is very difficult to find. That was something that made an impact on me.

As a society we are technologically oriented and it has removed that sense of camaraderie between us as people, you know like with the nurses there having that time to bond.

The students addressed the issues of people reaching out to each other regardless of whom they were and where they came from. Students felt that people reached out simply because we were there.

\section{Valuing Others}

A second common thread amongst all informants that emerged as a major category I named "Valuing Others”. To "value others” emerged over and over again as participants were overwhelmed by the feelings of human to human contact. Participants said that there was a personal connectedness that "just existed", a bonding that was there at the beginning and just kept building and building. Valuing others went beyond culture, country and language. Participants described a human closeness unrelated to role. It arose out of all kinds of intracultural and intercultural contacts from initially meeting someone, be it a professional colleague, a patient, a neighbour, a community member or community leaders. Participants said that all parties involved were remarkably open as human beings, relishing the meeting of others and wanting to learn from each one. Informants experienced life as it was locally lived and professional endeavours consistent with the immersion culture. Each person was unique as an individual but together as human beings. This theme was repeated in health provider/recipient situations, with casual acquaintances and with friendships that were made. Many of the students still maintain a long distance relationship with their professional counterparts as well as people that they met in social situations. Human closeness permeated daily living and interactions by sharing, supporting, story-telling, and learning about each other's lives, aspirations, families, and developing strong bonds between individuals. 
"Valuing Others" was the second category thread that emerged from four themes that were identified by participants. Each theme was derived analysing informants' stories. "Valuing” was a phenomenon described as transcending language. Informants stated that their communication went beyond words, as in many instances there was not a shared common language. It was reflected in simply being a human being, was exemplified as caring about and for others and was best captured in the sharing of lives across ethnic, economic and social differences. In the hospital and community health care settings, all levels of health care providers and ancillary personnel were delighted that students would come to their country to learn and work together with them in their institutions. Participants talked about their living accommodations and how their neighbours wanted to get to know them. The lack of a common language was not a barrier as students and family members talked, gestured, mimicked and pantomimed concepts. It took very little time for participants and those they visited to become "fluent" in understanding one another and the nuances of a conversation without the benefit of a common spoken language. Participants repeatedly discussed the openness of those they met and they also talked about their own openness and eagerness to absorb all that was possible in each new environment.

The first theme to emerge I named "Beyond language" and it was clearly described by Nicole. It involved her ongoing provision of patient care with a 77 year old man who was hospitalised in the last stages of amyotrophic lateral sclerosis [ALS]. Nicole spoke about how this one hospital experience deeply affected her and continues to influence her nursing practice today. The focus was that of communicating without verbal language but with one's eyes.

I remember one thing specifically and it was one of the first times in hospital and I had gone to a medical ICU. The patient that I worked with had ALS. It was my first day and I remember going up to him and smiling. He smiled, you know he tried to smile but he didn't have a lot of control of his face and his facial muscles and I had to turn away because I got tears in my eye. Here was this older man that was someone's father, someone's grandfather and he was, just, you could tell looking at him that mentally he was there but he had no control over his body. I vividly remember that experience. It gives me goose bumps thinking about it now because in the next few days I would give him a bed bath and do other activities of daily living and 
I'd come and I'd hold his hand and little things like that and there was no verbal communication and the only physical communication from him were his eyes, because he really had no control of his muscles.

I remember he had grey hair, not a lot of hair but I'd comb it and wash his hands and wash his face and he cried a few times, you could see there were tears coming out and I'd wipe his tears away. So that is one memory I have... and I couldn't even talk to him. I wanted to talk to him. He couldn't understand what I was saying but I would tell him what I was doing in English and probably just from the sound of my voice he knew that I was a friend. It was wonderful that I could help him and we could have that bond...

Jasmine similarly described her feelings and interactions with children and adults with whom she could not verbally communicate. For her "Beyond language" meant meaningful interactions with patients as well as interactions in social situations.

It was really incredible. The children who lived in the orphanage and those that lived up the street, how much fun we all had together. We played games and walked in the fields right from when we arrived until we departed. We were just being people, with that difference that we did not speak a mutual language. We got by with the kids as well as the adults. The bus drivers, the folks we met in the streets, everybody was so helpful. We didn't even know what the other person was saying yet we were able to communicate and learn from each other.

At the maternity hospital too, I remember being with labouring women and not knowing what they were saying. I could tell by their facial expressions what they were experiencing. Just being able to be there for them, holding their hands, touching their shoulder or something like that was very meaningful. There is so much you can express without words, without knowing the words. Those were really powerful experiences.

In the hospital people can't always verbally communicate for one reason or another; it is beyond them. The power of touch conveys a lot so that is something that I always try to incorporate in whatever setting I'm in. Holding someone's hand while you're talking to them, just something to let them know that you are there for them and I think they can really feel that.

Gigi's feelings on "Beyond language” played out in this way. She felt overwhelmed by the following scenario, a scene where a spoken language would not 
have relevance; it was "one picture is worth ten thousand words” and this so deeply affected her. She stated it in the following way.

I remember seeing all these women in this one room and they're all just sitting around making sounds of active labour. There were people coming in and out, kids rushing around and men walking in the room but mostly it was women in there. I thought to myself this must be the waiting room, and it actually was the labour room and everybody was in there labouring. One by one they'd go down the hall and lie on the metal table and give birth. It wasn't about making women's labour experience enjoyable; it was just like let's get her on the table, get the baby out and off she goes. I felt that there wasn't a labour experience.

"Beyond language” illustrates the depth to which students were affected by human understanding. Participants also talked about the power of communication, sans a common language and the ability to express/convey feelings.

"Being a human being" was the second theme to emerge. Nicole said of her clinical practice:

Your background or history doesn't matter, it's just that this person is a human being. You're a person first and because his diagnosis was ALS there was nothing we could do for him with that diagnosis but care for him as a human being. It helped me to realise that people are in hospital with whatever their condition may be but they're there as people first.

Natividad described being a human being, as being non judgmental, as giving up everything you have (for others):

I was really surprised at how non-judgemental and accepting people were about us. It was amazing to walk on the streets and have buses stop and people stop and say, "You are the Americans!" We had press interviews all the time! But beyond media coverage, people said they knew we were Americans because we smiled, we laughed, and these same people - from the street, from the hospitals, from everywhere - would invite us into their homes without even a thought as to the history between our two nations, or even how their homes might be perceived as what in America would be considered meagre accommodations. They gave everything they had. [name of country] are known for being incredible hosts and for loving to give gifts, but they really gave everything that they had. It tugged at my heartstrings, their love of sharing, and when I thought about what they might be giving up to take care of us. It was never about bragging or gloating or competition. We had beautiful tea parties and cakes and everything from that to lavish parties or just small get- 
togethers where we would share music or art. We were invited into people's homes like we were one of their own. We were not greeted and welcomed on a one-time basis like, "Hey, we did our part to show the Americans a good time." We continually built these relationships with the people that we met and became a part of their families and they became a part of our lives. I was just really shocked. I always believed in humanity. I've always believed that we're all the same, we all need love, respect, and we all need these basic essential things and that's what brings people together... When I looked at the people who were around the table with me, sharing food...I felt truly, like we knew that there is a universal thread that ties people together.

Alice described being a human being as simply being embraced:

It wasn't just like visiting a country, it was actually being immersed in the culture. We were drinking their vodka, sweating in their banyas, swimming in their ocean. We looked at their family pictures, met their children and were embraced like family members.

"Caring about and for others" was the third theme to emerge in the category of "Valuing Others". Jasmine described honouring the uniqueness of each individual despite having similar life needs. This is what she said:

The entire experience was really very eye opening. The interpreters met us and I remember one of them saying that she had imagined us as all being tall black men because their view of Americans was from basketball. She was really surprised that we were all different. There were a variety of cultures within our group as well as physiologic differences, you know average, short, tall, thin, heavy. In that sense it really broke down stereotypes and different pictures that we had of each other. It really just gives you the realisation that we're all people who have basic needs who are brought up in different societies and cultures and have different ways of meeting those needs. Realising that the interpreters were women like us and relating to them about common challenges that one experiences, those that happen everywhere, not just in our world, brought us a lot closer. I remember also how the whole immersion programme made it seem like a small world where people cared.

The theme "Caring about and for others" was also discussed by Lee as he talked about patient care. Caring was reflected as being concerned about another's well being in both their emotional and physiologic states.

We cared for people of multiple nationalities and spoken languages. Even though I didn't speak any of the languages they could tell by my body language, my interactions, my simple human 
touch that I cared; whether it was to hold their hand and talk to them or tell them that I really cared when I was doing things for them.

They could tell that I really was caring.

Trae was deeply affected by a pregnant woman who, accompanied by her husband, had walked for hours crossing a national boundary in order to seek health care. She was in a state of fulminating pre-eclampsia with a blood pressure of 240/160. This was not the first time the woman had experienced this extremely dangerous condition of pregnancy. Trae was impressed by the parish priest's actions when he anonymously provided the money for the woman's husband to have a vasectomy so that his wife would not have to undergo a future pregnancy. Trae stated that this was evidence of true caring. Caring about and for others was repeatedly reinforced in a culture far from what she knew/experienced:

I feel an expanded sense of compassion. I see the cycle of birth and death within life. The impact of the baby's miraculous, healthy birth (after not being able to hear foetal heart tones) gave me hope for a better world. This experience just made me feel good about humanity. That the priest would feel the way he did surprised me. I am happy that he cared and wanted to do something for this woman, that he believed would be right for her, even though the procedure conflicted with church doctrine.

Joan went on to say that whenever she or any of her fellow participants sat on concrete there would be immediate concern about their health. The locals would show their "Caring about and for others" by trying to protect the students from disease:

If they saw us sitting down on concrete they would come and they would find anything, they would take off their sweaters, they would find a piece of wood on the ground somewhere, anything to put underneath you so you wouldn't sit directly on the concrete for that was considered to cause illness and they were always concerned about us.

The last theme in the category of "Valuing Others" was "Sharing lives". In the interviews, participants talked about how their lives and the lives of the people and health professionals in the host communities became deeply entwined. From the give and take in their everyday lives grew an awareness and understanding of the 
universality of daily living, both the mundane and the special events. For instance Joan talked about sharing lives as follows:

At mealtime you were given so much food. It would never stop. Anything you put in your mouth, you look down and there was something else on your plate. Every sip you had they put another gulp in your glass and you were just so full at the end of the meal. They were all so kind, giving and welcoming.

Kaitlyn described sharing lives in a more detailed social context as she spoke about her fellow students and the circumstances of their bonding processes:

In terms of human interaction, dealing with the many challenges and multiple differences in life style made it an incredible bonding experience for all. I think that's why we're still friends even though we all live different lives in different areas. Because dealing with the emotional impact of the different culture and dealing with the emotional impact that we had on each other with effective leadership had allowed us to value each other's lives even though they're different.

All the students stated that their bonding experiences meant valuing one another, whether it was members of the community, their fellow students, their patients, and/or the health care providers. "Valuing Others" went "Beyond language” to simply being a human being with others. "Valuing Others" meant caring about, and for others, and sharing lives as mentioned previously. "Valuing Others” was a common thread addressed by all participants.

\section{Transforming experiences}

The last major category, “Transforming Experiences” emerged from the analysis and identification of four themes: “Taking risks”, “Assuming advocacy roles”, "Recognising prejudice” and "Having life changes”. “Taking risks” was expressed in many different forms; learning a new language, trying different foods, caring for patients without supplies and being flexible, adaptable and innovative. “Taking risks” also had an affective component that enhanced personal growth. “Taking risks” was expressed in many ways. For Jasmine it meant increased awareness as she moved beyond her comfort zone.

I read a quote the other day by John Lennon, "Living is easy with eyes closed, misunderstanding all you see." Again I just wanted to 
say that I am so thankful for the opportunity to have been immersed in the culture of a developing nation. I believe that the first step towards creating positive change is awareness. Once we become aware of our limitations, stereotypes, or lack of flexibility we can do something to change them. That is what the international nursing programme facilitated...we were removed from our "comfort zone" with all the amenities we take for granted and we were able to experience life under different circumstances. The experience not only opened my eyes to a different culture and lifestyle, but it increased my self-awareness as well as my acceptance of others. I think this lesson is extremely valuable and I have carried it with me in both my personal and professional life.

Joan talked about the programme as a transition between her childhood and adulthood and expressed it as the parallel transition from dependence to independence. She felt that the immersion experience affected her professionally, personally, intellectually and socially and that many seeds "sprouted" from her experiences. She stated that it was a huge life changing event and "Taking risks" was a key element in the process:

I think it's very significant to be able to step outside of what feels comfortable because the first time you do it, it's terrifying. It is so terrifying. And it's so painful and you feel like you never want to do it again and then it gets better and so it makes the next time a little bit easier. Soon you start taking more risks and even the risks become more comfortable and it broadens the way you think and it broadens the things you know and it broadens your experiences so that you can learn so much more. You just want to be able to get in there and have the most positive impact on your patient as possible. And when I say positive I mean positive for them and positive for me. I mean beneficial for them so that their health can be heightened and their experience can be a good memory for them. And to be open minded with that requires taking risks. I walk in and see a woman doing something really 'weird' in labour and some nurse might want to just get her back on the bed and say what are you doing. I just stand back and watch. I want to see if it's working for her. It tends to have a lot of really good results for me as far as building rapport and learning about differences.

Bugs said that she had never really travelled anyplace before that would have been considered a developing nation. She said that she grew up in middle class America (USA) in her own "clean little bubble". She also learned that in the global sense that sterile view gave her a false sense of reality. She assumed beforehand that 
the majority of the world's people lived, materially, much like she did and that health care institutions were similar in their physical plant, supplies and technology. But for her, the bubble burst with her participation in the immersion programme. She felt that this was beneficial for her as she was able to emerge out of her own little world. Bugs realised that most of the world does not have the many creature comforts that she has always taken for granted. The health care services that she was accustomed to giving and receiving were also quite different than she expected.

“Taking risks” for Bugs was working through some terror:

Before we left home I'd been practising to keep my mouth closed in the shower and to be very conscientious about every time I came near any water that came out of a faucet. I was doing this at home in order to condition myself. Anyway the first night we were in and I was brushing my teeth while discussing, with my roommate, the fact that you couldn't drink the water that comes out of the faucet. I had my bottle of water at the sink and as I went to spit into the sink I scooped out a big handful of water. I put it into my mouth and started swishing it around and then terror struck. Oh my god, what do I have in my mouth and I spit it out and gargled with a strong antiseptic mouth wash and drank grapefruit seed extract and did everything I could in the hope that I would be able to kill everything that might be in my mouth from taking that sip of water. I don't know if that's where I got my intestinal parasite, it could have been but who knows. It's a different way of life and it was good to experience that.

Lee talked about "Taking risks" in the arena of patient care. He felt that it just wasn't the refugee patient population that was powerless but all of the patients. Routinely he described how the medical staff would go to a patient's bedside and talk amongst themselves without including the patient. He read fear in the eyes of the patients, as they were not informed of their status, condition, treatment or prognosis. He talked about taking risks with patient care by breaching secrecy codes. He felt patients should know what their present health status was, in a culture of medical practice that valued them not knowing. With knowing, Lee used compassion:

I tried to explain, in ways that they could understand, what was happening to them. I couldn't speak the language; I would just try to show them with my kindness, my care, caring, touch, and my focus on them. 
Another theme under the category of "Transforming Experiences" was “Assuming advocacy roles”. It was demonstrated by Lee, but verbalised by others as well. Participants described advocacy as having far reaching effects in both their personal and professional lives. Advocacy involved speaking out and acting on issues on behalf of patients; it involved support, help, encouragement and providing for the physiologic and psychosocial needs of the patients.

Kaitlyn talked about an advocacy role she assumed (current practice) for an elderly woman that had sustained a heart attack and was brought to the hospital emergency room by her extended family. When she was stabilised she was admitted to the ICU (Intensive Care Unit).

She wanted to go home. Her family wanted her to go home. It was so clear that she wanted to go home and the doctor would not allow it. I felt that my role as a nurse and as a patient advocate, no matter what I would choose in similar circumstances, I needed to step in and advocate for her wishes. I was so proud that day because I was able to step in and insist that the doctors listen and they were finally able to say if you go home you might die and she looked at them and she said I understand that. I want to go home. That was a day I left work and I cried. I wasn't crying with frustration, I was really happy to be able to be in that role as patient advocate.

In my practice as a nurse I feel it's made me a more effective patient advocate to not be self-righteous and have all the answers. It allowed me to listen to my patients and to glean what they say and what they feel they need. And it may be different from what I would feel I would need in the same circumstances but what I feel I would need is not important in that case.

Kaitlyn felt that she learned advocacy skills are transportable and have enabled her to continue to become an effective nurse advocate.

I used to feel that I had the answers and that I knew how to cure the illnesses of the world. During participation in the programme I realised that I didn't have answers and those that I had wouldn't necessarily work for someone else. I realised that the way I lived my life couldn't be imposed on the people that we were working with and in some ways that's a very uncomfortable place to be. At that time, I was older living for 35 years with this sense of absoluteness. I knew what the answers were; I had my opinions. To have your preconceptions challenged is difficult. 
I feel very fortunate that I've grown comfortable with not having answers. In my practice as a nurse I feel it's made me a more effective patient advocate to not be self-righteous and have answers. It allows me to listen to my patients and glean what they say and what they need. It may be different from what I would feel I would need in the same circumstances but what I feel I need is not relevant.

Natividad focused on the advocacy role in the framework of the immersion programme commenting that every nurse needs to enter a culture that is unfamiliar. She talked about her early life and the guardedness that resulted from her leaving home at a young age to fend for herself. Early on Natividad considered herself shy and unable to share of herself due to her perceived feelings of vulnerability. But as she began to experience a different culture, she was finally able to open herself to personal and professional change.

I was just really shocked. I've always believed that we're all the same in our need for love, respect, and the basic essential things and that's what brings people together. However you grow up with all these stereotypes about I mean, they were the Communists, the people that we were against in the Cold War and the secret police were supposedly everywhere in the eighties - you hear all this stuff growing up.

I have to admit that it almost sounded like an ideal utopian fairy tale when I told people about our experiences in It was really hard for people in the States to believe what had transpired. It was also difficult for many people to believe that this kind of programme makes a difference. People would ask me, "Oh, you are going there for a couple of months, what kind of sustainable change are you actually producing from this? What kind of outcomes are you going to see?” You know we carry it with us everywhere we go. The sustainable change lies in the fact that we were accepted, that we broke down the stereotypes, and that they know Americans, and we know , and whatever the biases might have been, we brought reality and humanity to one another.

I can identify that I have a dramatic change in the way that I look and deal with people, how I really live my life, but sometimes you can't really verbalise what you mean, you just know. You feel it in your gut and you feel it in your heart and you know it in your mind. It's just that way.

This is an opportunity, this kind of program that you [MaryAnne] have provided with your blood, sweat, and tears at this university. It is the kind of opportunity that not only every nursing 
student should have but every person should have because, personally, you want to know that you can deal and provide in all types of situations and environments and be flexible and be adaptable.

Gigi identified her advocacy role as one of the ways that she views her nursing practice today. Working in a developing country introduced her to nursing care minus all the amenities one is used to in the developed world. She also talked about how individuals do not realise how different life is for people in their little corner of the world versus one's own world:

It's the polar opposite of where we live. You see things more for what they really are. I don't know if I really saw it while I was there, but after I came back there were many realisations. You get absorbed in where you live and become so accustomed to a certain way of life that you don't think about other places and how other people live.

People are always making fun of me at work because I am very frugal with supplies and things. I have this tirade when I go into a room and there are three or four mouth washes and all this stuff in the drawer and it's going to go into the bin when the patient is discharged. I'm constantly asked, what are you worrying about? I always remember being in and having several experiences that just blew my mind. I remember going to the ICU and watching glass syringes being washed after use, then dried and reused again. This was the main hospital in the city where we worked. I remember saying, oh my goodness, to myself.

I remember the rubber gloves; it is just stuck in my brain, hanging out on the laundry lines to dry. I have a picture of that and I think, my god, how many pairs of gloves I go through at work every day, just constantly throwing them away, throwing them away, throwing them away. So I do tend to be more frugal at work and people make fun of me in that way. But I think that that is something that stems from having seen people practice nursing, midwifery and medicine in a place where there are just no supplies. I have become an advocate among my colleagues related to judicious use of equipment and materials.

“Transforming Experiences” also dealt with the issue of recognising prejudice, and its existence in both blatant and subtle forms. Participants discussed their realisations about themselves and others having preconceived notions about people. They discussed their own discriminatory beliefs and biased feelings that they did not realise they held. This enabled them to change. As Jasmine said: "When we 
become aware of our limitations and stereotyping we can do something to change them..."

Feelings initially surfaced as indignation that indigenous people were not considered as worthy as others and were treated with disdain, rudeness and intolerance. Stereotyping also arose as refugees were thought of as "lazy" and "not wanting to work or contribute to the society in which they now lived”. Other instances of intolerance emerged as people of a particular ethnicity were grouped and isolated. Certain attributes were often ascribed to all the members of a particular group of people. Students saw prejudice manifest itself both in the hospital and in social situations.”

Joan talked about a typical example of prejudice concerning a preterm neonate and his mother in the maternity hospital newborn nursery. The mother was a member of a minority ethnic group and was treated markedly differently than the patients of the majority population. She was ignored, she was not spoken to, she was not offered routine care and the health care that she was given was substandard. One day she simply walked out of the hospital. Once a week she returned to visit her preterm baby. When she left the hospital personnel kept reiterating among themselves and to the students, "what can you expect from [named ethnicity], they don't care about their children. They're dirty, they don't want to work for a living and they are uneducated.” Joan described this typical incident:

The mother came into the nursery to visit her baby. The paediatrician was there and just ignored her. The mother kept trying to get her attention and finally the doctor turned around and yelled at her and somebody translated for me that she was saying you can't just walk in here and expect us to help you we've got other things to do. Anyway I went and brought her the baby and tried to help her nurse the baby and I just remember how ostracised she was and it's interesting because we have that in this [USA] country, definitely. That same level of disapproval and judgement of people who are different but there it was so much more obvious. Here we're kind of socialised to keep it quieter, especially more educated upper class people. Everybody still has the same prejudices and judgements but we express it more subtly and it was quite obvious there and it was disturbing to see the way she was treated. I had never seen anybody that obviously told you're different, you're wrong, we don't like you, get away. 
There was a part of me that was really hesitant to step in because I knew that on one level this was their culture and I was a visitor and what gave me the right to walk in there and go against what they were doing. On another level, being separate, being a foreigner and being external to that culture, I felt more freedom.

Joan went on to talk about how this has subsequently affected her practice.

I make sure that every woman is treated with respect and honour, honour in her motherhood, honour in her bond to her baby and without judgement of whom she is and where she comes from. In this way it has definitely influenced my practice. There is a lot of judgement by the nurses and doctors: she does drugs, she's a bad person, she's a bad mom; she didn't get prenatal care. I see it all the time in the hospital. My immersion experience definitely taught me to treat every client with respect.

I often get the patients nobody else wants. I will come onto shift at night and they will say "I want you to take this one" and usually it will be a teen mom, a Spanish speaking mom, a patient that the staff defines as difficult, a mother who uses illicit drugs, or just a mom who is driving everybody crazy.

I remember one night there was a lesbian couple who had a baby together and the nurses were laughing at them at shift report and saying that they were disgusting and that they were in bed together, and it's so gross. They're sitting there and they're kissing. I volunteered to work with this family and they laughed and said "no we're going to give her to [name of nurse] a nurse that nobody likes. They wanted to do this because she drives patients crazy, because she is perceived as talking very loudly and being very annoying. They were basically trying to offend this family by giving her the nurse that nobody liked. I said "no" I want to work with them. I want to make sure she has good care, and is treated with respect. I generically gravitate to those patients who are perceived as different and do really well with them and enjoy working with them.

Lee addressed recognising prejudice this way.

From a personal standpoint I felt seeing prejudice in action made me a better and more educated person by just experiencing the multiple cultures within the one country. Working there made me less biased. It's everywhere and it has becomes less significant for me as an issue in my patient care. I just focus on people. I went there with preconceived ideas that there were no biases and learned no matter how things are different they're still the same. It made me feel more confident in my interactions with people and I learned that we are all 
individuals, not just a religion, nationality or race. I was well into my 40’s when I went and yet I was able to make a big life change.

Stereotyping is so common by race, religion or colour and with preconceived ideas about behaviours and a certain way of life. Today I go into a situation totally accepting the patient. I grew up in the American south, a very biased part of the United States and when I returned from the immersion programme I really felt that I had made significant life changes. It was almost like a spiritual awakening about the reality of life and how people are. It was a great experience. You just look into the eyes and soul of your patient and communicate with them. We're all people, and if we can just break the chain of bias and racism it sure would be a better place.

Kaitlyn talked about recognising prejudice in a rural setting of a developing country. It was a difficult and painful lesson.

The practitioners, the doctors, the nurses at the Catholic hospital were absolutely wonderful and dealt with their patients with respect and kindness and patience. The practitioners in the public hospital were quite a contrast. The indigenous population, were treated by the doctors as less than human. They were talked about in front of them as if they weren't there; they were talked about snidely and cruelly during surgical procedures and the doctors even made crude jokes. The doctors were speaking Spanish and they made the assumption that as 'gringos' we could not understand, but we did. In a particular instance they were juggling a woman's uterus and making jokes about turning it into soup. We were very scrupulous about being polite and biting our tongues, but when we left and were returning to our residence, we cried the entire way.

That evening at our group meeting we needed time to decompress. It was such a shock to our system to see that kind of [personal] disregard. We knew that there is much disrespect in the world but to experience it particularly in a profession that is supposedly a caring, healing profession was shocking. It made me absolutely determined, in my practice as a nurse, to always be sensitive to patients. I resolved then that I would always exercise patience and tolerance to all people.

Students described how one may not be aware of one's actions toward others as being discriminatory, unfair or biased. Recognising prejudice was an extremely painful process for students to experience. At the same time it brought home the realisation of their own prejudices. 
Nicole talked about her own prejudices as "self-revelation”. She described it in the following way.

This nurse that we had been socialising with came to pick us up one day and she looked at us in a strange way. We were outside hanging around with the kids from [geographic location] and she asked us what we were doing; she thought it was odd that we would be playing with these children. I remember thinking these people are not accepted into the culture. I mean they are out here, they're great, families with many children all crammed into one room. They were refugees who had to flee their own country in order to save themselves from the war that was raging about them.

It was a strange feeling the community was so accepting of us, yet these refugees were not at all welcomed. The [name of a refuge group], letting us play with their children without knowing us and smiling at us and trying to say hello and then having the nurse and her family thinking that it was not proper that we were associating with these people. It was so shocking because we didn't want to offend our hosts who were the nurses we were working with, but it made no sense to us that these people were not okay to play with.

The local prejudices were coming into play, of which, we had no knowledge because we were from out of the area. That was interesting because I then realised that I have my own prejudices that I don't even think about but all of a sudden we were put into a place where we didn't have them and so coming home that made me think, why do I have these prejudices and it's a big thing.

Last week we had a patient from [geographical location] that is about 120 kilometres from the hospital. It's up in the hills and the [racial grouping] come into town for health care when it's serious. We had a woman who was severely beaten. I don't know the whole story because you never know. She was bruised from head to toe and there was a nurse new to the area working with us and everyone said oh well she's from [geographic location] and everyone said, okay, I understand. The new nurse didn't understand, as she was new to this area, and it didn't make any sense to her. And all of a sudden, oh my goodness, I realised that we were stereotyping this woman because she was [racial group]. The new nurse didn't have this frame of reference. Thank goodness that new nurse was there because she didn't know. Like when I was in [name of country] I didn't know that the [name of a refugee group] were not okay to be with. 
I still think about it, and get caught up in it, but hopefully I will remember to think back on it. Those prejudices are all learned; they have no basis. This patient was a woman and I don't know why she was beaten up and I can't make assumptions and it doesn't matter where she was from. We all assumed because she was [race] that she was a dysfunctional person.

For all the participants, the issues related to recognising prejudice had profound long term effects.

The last theme was that of having had life changing experiences. Participants talked about ways in which the immersion programme transformed their outlook on life professionally and personally. They discussed their increased feelings of comfort in working with diverse people and realising that all individuals have their unique ways of being. They talked about being open and flexible and of relinquishing control to their clients who knew best how to restore their health, achieve their goals and meet their expectations. Participants talked about how they have incorporated experiential learning into their nursing practices.

Alice said:

The experience in and the experience of working with other cultures has made me feel like I can go anywhere and I can work with any people. I realize when patients come to my hospital and they walk in the door that they have a whole different thing that they're bringing with them. Not just the fact that they're here to have a baby or to have surgery. They bring so much with them when they come in the door that is so fascinating and so exciting and if we just give them time to tell us about it, we can then provide care in a more satisfying way.

Alice felt that it was critically important to learn as much about the patient as possible to ensure the best nursing care could be provided. It wasn't a matter of “things” or technology. It wasn’t about supplies.

Gigi also said that the trip made her realise that material possessions were not so important:

It makes you think differently about yourself and your place in the world. I have less appreciation for material things; they just aren't that important. It really had a big impact on me seeing how people live with so few material possessions. Seeing families that were happy and smiling and having fun and children, too, who had only a 
stick or a root to play with. As a parent that has made a profound impression on how I am raising my two children.

Trae learned to understand the differences in people related to their perceptions of health and healing. In her practice now she consciously promotes individuals incorporating their own belief systems. She continues to enjoy participating in cross cultural practices that are different from her own and she values traditional practices and ancient ways:

My international immersion experience has helped me understand and promote the cultural practices of other people. I have a better understanding of how people view healing in different ways. Going to the hospital is only one option. I like to work with patients in valuing their own historical and cultural practices especially after seeing how so many people in were ready to abandon their own cultural practices and embrace those of the United States. Mothers were ready to bottle feed their babies with formula rather than breastfeed, even though breastfeeding had been part of their culture for aeons.

I am comfortable with the rituals and practices that my patients care to use. I like to participate in those rituals that I'm invited to join. If I'm not included, that's fine. I keep a respectful distance. I feel that these are very healing for the families. I feel that being immersed in a culture so totally different can't help but broaden your experience, it's impossible not to. That when you see health care from a totally different perspective in a small village in mountainside communities where people just don't have the access to services that they do here, that you have to - for want of a better phrase broaden your heart, widen your perspective. And even more, you need to be more accepting of what other people want, of the way other people view their health care, and more understanding of what they're going through.

Jasmine talked about her life changing transforming experiences as being "humbling". She discussed how she was able to transcend her own understanding and view life from a different vantage point, realising there are many ways to live:

It gives you an appreciation of differences. You know we get this view of certain things in our life and then step out of that view and learn that there's a whole other way of living life and ours isn't the only way to live. It's a learning process and it puts you in a place of humbleness and awe. I mean the ways of seeing the different things and being blown away by them... 
It's definitely a life changing experience to have the opportunity to participate in the immersion programme. It opens your mind and your eyes and it totally reinforces the fact that we're all people and we are all just trying to get through this life with our different circumstances. Well, I'm really thankful that I had this opportunity because it does awaken you to what's out there in the world more, there is just so much. There's little pieces here and there but it opens up something inside of you that makes it easier to continue to stay open to individuals and their uniqueness.

Maybe there is something that makes you inflexible and when you're forced to change every way that you think is right then wow, that is mind opening. That widens your tunnel vision that we get caught up in. You've learned a lot, your mind has been opened and there are ways of being open to others and accepting of others, that's the important part.

Something else that I want to bring up, our technological orientation has removed that sense of camaraderie between us. The nurses in have time to bond with one another whereas here it is not so common.

Alice described how her practice has been shaped by her life changing experience:

Whenever I work with somebody from a different culture and I don't just mean other countries, with a person who could be deaf or the person could be Amish, I step back and allow them to bring me in rather than enforce my values on them. I make them much more at home and I've had very good responses. People have been very happy with their care and I feel that it makes a difference to them. That somebody cared enough to know about them as an individual/family and what they wanted their experience to be like, what their spiritual and cultural values are, and how that affects their hospital experience...

It makes me feel honoured and grateful that clients are willing to share with me but also it makes me more confident as I am not enforcing my values. They're letting me know what they need and I make it happen.

Bugs had her set ideas about the world completely challenged; she felt that her world was turned upside down. She also felt participating in an immersion programme broadened her perspective and changed her modus operandi in the following way: 
I think about the trip quite often and how fortunate I was to be able to participate. My sense of the world was definitely broadened; to see, to feel, to hear and experience things that most other people won't ever realise. I didn't really know what the rest of the world was like. I haven't been anywhere else to experience how life is in other parts of the world and I had my set ideas about social interactions. I had my ideas of how health care should be delivered and so all of these ideas were certainly challenged. The way that women are treated was something that I had just read about and I knew existed but I had never actually seen it. My whole world was turned upside down.

The programme has impacted my practice in terms of how I interact with patients. I try to always be gentle and not touch anyone in an abrupt kind of harsh way and to be slow and to make sure that I understand what the patient is wishing to express, or is afraid to say. I build trust so that the client can feel comfortable to express their feelings and needs. If someone expresses to me that whatever it is that we are doing is in accordance with their beliefs I have respect for that and advocate for them in the whole grand scheme of their health care.

The three major categories "Having Blind Trust”, "Valuing Others", and “Transforming Experiences” emerged from the process of identifying common themes that were addressed by all the participants and represented the topics and issues that were discussed during the interviews. The respondents were sent copies of what I have placed in this thesis in order to ensure accuracy of their identified themes and all have responded in the affirmative, that I have accurately portrayed what they told me. The common themes, patterns, and experiences that surfaced over and over again in the interviews were placed into three categories. No additional themes emerged. I was truly surprised by what the informants had to say and at the commonality of feelings that arose.

\section{My Voice}

As I think of "my voice” I reflect on the voice of Moustakas (1990) and his interpretation of the concept surrounding the word heuristic. A word that ...meaningfully encompass[es] the processes that I believed to be essential in investigations of human experience. A process of internal search through which one discovers the nature and meaning of 
experiences and develops methods and procedures for further investigation and analysis. The self of the researcher is present throughout the process and, while understanding the phenomenon with increasing depth, the researcher also experiences growing selfawareness and self-knowledge. Heuristic processes incorporate creative self-processes and self-discoveries. (p. 9)

Throughout this thesis the research questions and the heuristic process of Moustakas (1990) guided my work, thoughts and thinking. As I reflected yet again I asked myself: "Why do I do this work? "LET ME COUNT THE WAYS” (Browning, n.d., p. 43).

Each of us has a signature modus operandi. I am who I am, in each of my many roles. Duhl (1963) said “one’s own style as person obviously is the core of one’s style as therapist” [teacher, nurse, midwife] (p. 76). I don’t change persona, for I am very comfortable with myself. I delight in each and every day as it unfolds: the nuances of daily living that are "just there"; the challenges that I will take on; the joys of life that surround me; and my multiple roles and functions that play out into unique scenarios that engulf me. I am constantly nudged to learn about the way of others, as if it is an imposing "artistic process". My experiences intertwine with others. What has brought me to this place is not a paycheck but a genuine passion for my fellow human beings, travellers together in this life of ours, in all our similarities and differences.

I am the product of my socialisation process, an extended family that had the wisdom to instil in their children a dual world focus, both an internal loci and an external orientation. My parents always encouraged, and had us participate in the world of the other. I have always known comfort and understanding in the uniqueness of individuals and groups, recognising the similarities in differences. I look upon the peoples of the world as just an extension of my family, the human family. My own psycho/socio/cultural growth continues as each day I interact with others and continually gain new knowledge of the ways of fellow human beings.

As a teacher I realised that I needed a tool for students to understand these similarities in differences. I also realised that I had feelings of sadness at a country, an education system, that doesn't prepare its citizens for life, by addressing people in all walks of life, ethnicities and colours, as individuals with feelings, aspirations, 
dreams and hopes, similar to their own. I needed for students to feel the humanness of all the people that they worked with. I needed for students to use all their "parts", bio/psycho/socio/cultural, so that their world view would expand. I needed for students to become provocative beginning professional practitioners and know confidence and feel pride in their ability to use themselves as the ultimate clinical tool, the effective communicator. I needed for students to know who they were and what they bring to their role and functions as a nurse. I needed for students to look at issues of power and politics and their formidable cumulative effects on people across the life span, in whatever country they happen to live out their lives. I needed for students to understand, for themselves, what is meant by powerlessness and poverty.

I needed to find venues external to their home country so that each could "taste” powerlessness in their lives. In the USA students were part of the majority establishment, they were representatives of the power of the institute where they did their clinical rotations. In the venues of others the students become the "etic". They were now separated from the "system", the comfort and the safety of the familiar. They worked with midwives and nurses whose roles differed from what they had experienced in their home country. Students had themselves, their own resources, their critical thinking skills, their creativity, adaptability and their innovativeness to rely on and hone.

One may well ask, why take students internationally. Poverty and powerless can be found on every door step, in the inner cities and rural areas of every country, at homeless shelters, at clinics that serve the impoverished, at government hospitals that provide for the poor, in minority communities, on Indian reservations, on the streets and in the schools of culturally diverse non English speaking environs. This is true, but what is different is that students would still be members of the majority culture, part of the establishment and hold, as such, the "trump card".

I have practiced my profession in all of the above venues; I have had a gun brandished at me and a knife wielding parent of an abused child threaten me in a paediatric emergency room where I worked. I have taken home a four year old; he who was drinking water from the toilet, because his mother had been murdered 
several days before. The police had finally broken into the flat and he was discovered. I received permission from Child Protective Services to take the child home for the weekend. The hospital administration, however, was outraged that a nurse would do such a thing. Because of this I lost my job. I stood my ground as a human being, as a nurse, as a caring person and as a patient advocate. The administration rescinded based on the actions of other nurses and doctors who threatened to call a New York City tabloid newspaper and report their actions. I have bought food for children, learning through experience not to give the money to the parents but instead bring actual meals to be eaten, rather than the money being used for alcohol or cigarettes.

When collecting data for my Master's Degree thesis (Levine, 1977) I worked with two undergraduate nursing students on an Indian reservation in South Dakota (USA). One week prior to our arrival three FBI (Federal Bureau of Investigation) agents were murdered. We remained for eight weeks and this was a very tense time at this location. At this remote site we were still members of the majority culture.

For two years I worked with a Spanish speaking population in an inner city setting of New York City. My ritual became one of thumping up the stairs of the tenements to scatter the mice and rats. Upon arrival home I would divest myself of most of my clothes outside of my flat so that the many small travelers of the insect world would remain at bay. I was initially there as the other, a member of the dominant society.

Immersion into a country external to one’s own provides incredible learning experiences, the most important of which is the issue of powerlessness. Students can't return to “their world” at the end of the day or night; often they can't speak the language; the food is different; the ways of the people different and activities of daily living are in direct contrast to those of the students’ norms. Herein lies true experiential learning.

In the personal and social spheres students lived with local families and accomplished their activities of daily living as their "families" did. They became part of the extended family and were treated as family members. Depending on the venue personal bathing and laundry were done in the local river, laundry by hand on 
a rock. Toileting was either sitting or standing over a hole in the ground, or a purposely dug hole, surrounded by cement. Electricity was sparse, and unreliable, water was collected in barrels and then filtered for the purpose of drinking. Washing dishes could mean carrying a metal basin replete with the dishes to the river or the lake to wash. This began a growth process of learning about the other by experiencing "their world".

My students and I continue to get into animated discussions about childbearing women of poverty in the developing world as they find it difficult to comprehend events that are everyday occurrences in the lives of this cohort of young women. In the developed world there are graphic pictures in newspaper, on television, and there are many human-interest stories on the plight of poor childbearing women of the developing world, but "out of sight, out of mind”. For me, it is the recurrent theme that emerges again and again, the lack of knowing about the other and it begins at the basic level of "Who am I?"

My raison d'être: my youth, my socialization process, my life in toto, perhaps, I have viewed through my marginality, my marching to a different drummer, my activism, always going beyond myself (depending on your views and orientation some might label this as minding the business of others) and putting myself out there to "walk the talk". Perhaps it is making a difference one person at a time. Sharing a life, each person involved richer for the experience.

When I reflect on my experiences with childbearing women who live in poverty, I feel the pain, the loss of those who are treated as lesser individuals because they are powerless in their society whether due to poverty, gender ascribed roles, race, religion, ethnicity or colour. Those who are disenfranchised, nonmember of the prevailing culture are treated differently.

I remember being in a shop in a small town. I had just entered the store, the owner had just finished with one customer and an indigenous woman was waiting to be served. The proprietor turned to me and asked what I wanted. I said the lady next to me was here first and I was told, “she'll wait, I'll serve you first, she’s just an Indian”. 
Labour and delivery, the scenario plays like a recording, over and over again, at multiple geographic locations. I want to shout: “There is an entire person who belongs to that perineum.” A woman who has multiple roles...mother, sister, daughter, niece, aunt, tiller of the soil, market gardener, wife, daughter-in-law, sister-in-law, housekeeper, cook, grand-daughter, nurturer...a whole woman with intrinsic worth. She shares the same biologic functions as you, the care provider, and all the psychosocial functions, albeit, those of her "world". She does not have the education, the visibility, the power, which the health care system wields, in her daily life, or in this institution. Her vulnerability is pervasive and taken advantage of.

Her physiologic poverty is glowing... anaemia compounded by helminthiasis, short stature, and her weight. Her dress tattered and soiled. The dress, which she labours and delivers in, is the same dress that she will wear for the remainder of her hospital stay. The dress she'll wear, when she, carrying her baby, is sent on foot to her village a few kilometres from the hospital. The hospital bed is sans any covering, with the original plastic mattress cover that has multiple holes that she will soon be sharing with another parturient and many roaches.

The students are another reason that I return. The importance of exposure, and understanding of others in their home communities and a greater understanding of the global community are essential to the role and function of nurses and midwives in order to give the best possible care. It is now the $21^{\text {st }}$ century and still the power structures exist that continues with the rights of some and the nullification of the basic human rights of others. This is another reason that grounds me and causes me to return. I am compelled also, for are we not “our brothers' keepers”? For are not the people of the world just an extension of one's family?

\section{Summary}

This chapter has revealed the findings of the interviews with ten nurses as well as brief biographical information of each participant. The analysis of the interview transcripts produced multiple common themes that were then put into three major categories: "Having Blind Trust”, "Valuing Others”, and "Transforming Experiences”. My Voice was included in this chapter as it fits well with the students' 
findings, was parallel to what students said, and contributed to my own reflective process, ultimately to my epiphany. I found that immersion programmes were extraordinarily transformative for participants. The following chapter focuses on the analysis of the dialectics of the students. 


\title{
CHAPTER VI
}

\section{FINDINGS PART II: EXPERIENTIAL LEARNING AND THE DIALECTIC}

\author{
We shall not cease from our exploration \\ And the end of all our exploring \\ Will be to arrive where we started \\ And know the place for the first time \\ T.S. Eliot, Little Gidding V
}

(1943, p. 39).

\section{Introduction}

The research questions in this thesis address the essence of who I am. This work reflects my inquiry into a process that I long took for granted and which remained unexamined for many years. The results of the student interviews (Chapter V) and subsequent thematic analyses left me initially bewildered; I was truly shaken and astounded at the depth of feelings that students had revealed. As each discussed and recounted her/his immersion experiences, as each addressed her/his current nursing practice, and as each talked about life changes (directly related to their experiences in the programme) I realised that I was obliged to undertake a further level of analysis.

Qualitative research, in this project, was used to identify various common themes. It also, at times, unearthed diametrically opposed concepts, called dialectics. The term dialectic comes from the Greek language and means to talk, communicate, discuss and the "dialectic in Greek philosophy retains (the above) sense of the term; a conversational form of argument; a method of explanation; a style of reasoning (Powers \& Knapp \& 1995, p. 44). Hegel used the dialectic to signify “contradiction and resolution of contradiction through reconciliation of opposing entities" (Powers \& Knapp \& 1995, p. 44), the scheme of which is “thesis, antithesis, synthesis” (Barnhart, 1969, p. 561). Another way of expressing the dialectic is an “...original tendency, its opposing tendency, and their unification in a new movement” (Barnhart, 1969, p. 561). It is significant that a person who is exposed to "newness" 
in the sense of cognitive, physiologic and/or the affective domains perceives a push/pull effect and is able to look at and develop another view, through opposing expanded information. This new information, obtained through exposure, experience, and immersion will then coalesce into new formations, again in any or all of the domains, cognitive, physiologic, or affective.

Students were constantly exposed to differentness, to dialectics, in their nursing role and functions in the health care system, in their "socialisation" process within the communities, and in their personal relationship that they developed at the venues. For myself, I was in awe of students’ expression of feelings and their articulated level of awareness that constantly occupied their thoughts. I was constantly exposed to their dialectic(s), through their eyes, and additionally became aware of my own dialectic processes that unfolded at each venue through careful assessment of the vignettes. Our reality is our world view and one must stretch those boundaries and go beyond one's known world to a broader view:

The justification for much of what we know and believe, our values and our feelings, depends on the context--biographical, historical, cultural--in which they are embedded. We make meaning with different dimensions of awareness and understanding; in adulthood we may more clearly understand our experience[s] when we know under what conditions an expressed idea is true or justified.... Interpretations and opinions that may have worked for us as children often do not as adults. (Mezirow, 2000, pp. 3-4)

By undertaking an analysis of the deeper processes involved in this chapter I will first explore the dialectic for students and how they have been able to come to a new way of knowing themselves and their world through this process. Dodd (1998) defined world view as:

A belief system about the nature of the universe, its perceived effect on human behavior, and one's place in the universe. World view is a fundamental core set of assumptions explaining cultural forces, the nature of humankind, the nature of good and evil, luck, fate, spirits, the power of significant others, the role of time, and the nature of our physical and natural resources. Because it is so fundamental, world view affects communication encounters and perceptions of difference. (p. 95)

This means that the individual is powerfully influenced by one's world view that has been formulated and conceptualised through one's socialisation process. Through 
immersion into other cultures students were exposed to people who had diametrically opposing views, and thus the dialect was born. The dialect seeks "truth through the exploration of contradictions” (Cox, 1980, pp. 134-135). Anything one does that is new may lend itself to contradictions and then increase knowledge. I feel it is "my compulsion” to expose students to dialectics, as I have been exposed throughout my life.

I look beyond the traditional standard of communication patterns in the discipline of nursing. I envision communication as all encompassing in being able to touch, reach and (re)awaken the affective component in people, among them students, thus opening each to the greater world beyond their own. I endeavour to whet the students' appetites for exposure, understanding, and comfort in other cultures. While I can establish the exposure and the immersion of students, I cannot influence their own critical assessment. As "my control” ceases, it is the student and what he/she then does at the end of this process. "Faculty may not be comfortable with the unpredictability of this process, and particularly with the perceived lack of control over outcomes” (Carpio \& Majumdar, 1993, p. 7). This means that in a lived life "new beginnings" can take place through exposure, immersion, reflection, and communication with self and others, at one's home base and/or at multiple venues.

This is resolutely linked to one's modus operandi in the world and Habermas (1968/trans. 1971) succinctly addressed the "possibilities” of interpretation: "What does it mean to understand social actions? There is interdependence between the basic concepts of social action and the methodology of understanding social actions.” (p. 102). Thus, with exposure to "newness” and to people with opposing views, the dialect was readily evident. The implicit implications relate directly to society and interface with one's world view. This in turn directly connects to how one interprets and relates to the formal institutions of a given society and the warp and weft of the social system in relationship to individuals and groups.

Boutain (1999a) stated that nursing inquiry has "shattered some of the silence in nursing about social ills” (p. 1). She further stated that although nursing scholars continue to explore and acknowledge the definitive relatedness of social inequality and health status, there is much that remains to be done. Boutain's thesis 
was identifying "not which research questions to ask, but how to ask research questions that broaden knowledge about the interconnections among language, discourse, health, and society” (p. 1).

Ironside (2001) contended that the critical teacher "is committed to empowerment... and social action” (p. 76). Further, Ironside (2001) posited that no one is immune because "critical pedagogy denies the possibility of neutrality (every person is affected by his or her culture, socioeconomic position, personal history, and so forth), teachers and students alike are... implicated in the very structures they are trying to change” (p. 78).

The realisation that, simultaneously, I am part of the traditional teaching establishment and yet also using a non-traditional method to teach, guide, educate and prepare students, centres me. It also “incriminates” me for I do indeed have a vested interest in student learning by way of immersing them into the lives of the other, professionally and socially, in lands, languages and cultures unknown to them or myself.

This chapter will discuss the common dialectics of the participants to glean the most prevalent opposing ideas or conflicting principles. It's a viewpoint that enables me to describe "cold" by experiencing "hot". The data is taken from the same ten participant interviews that were previously used. There were three major oppositional categories:

1. Have and Have-Nots

2. Being an Insider and an Outsider

3. A World Shrinking, a World Expanding

A rigorous educational framework will be introduced next because it is the appropriate vehicle to address as it looks at individuals, their feelings and their way of looking at life and the potential changes that can occur from the introduction of new knowledge. It is a necessary and important component to this thesis. Made up of Diekelmann’s narrative pedagogy (1990, 1993, 1995, 2001) and Kolb (1984) and Vygotsky’s (1926/trans. 1997) theories on experiential learning, which guided my 
teaching approach, as well as the thoughtful and rigorous framework of Moustakas (1990) that added further depth.

\section{Educational Theories}

\section{Narrative pedagogy}

The research of Diekelmann (1993, 2001, 2003) has deep meaning as in part she seeks to bring together educational theory and the pedagogy of learning through every day dialogue. "Pedagogies are particular approaches to schooling, learning, and teaching” (Diekelmann, 2001, p. 54). She (1995, 2001) called her approach "narrative pedagogy” which has arisen within the profession of nursing itself. It can also be described as "interpretive pedagogies” which is “... a shift to critiquing, examining, exploring, and deconstructing the experiences experienced by students for their meaning and learning” (Diekelmann, 2001, p. 54). Diekelmann is most known for processes related to "the meaning of practices shaped by behavioral pedagogy within the day-to-day lives of students and teachers” (Diekelmann, 1993, p. 246), and "the common lived experiences of students, teachers, and clinicians" (Diekelmann, 2001, p. 53). Themes are generated to encourage reflection, discourse, intense discussion and interpretation that in turn creates new appreciation, new knowledge and new perceptions. Narrative pedagogy "creates possibilities for new understandings to emerge” (Ironside, 2001, p.8). science. Diekelmann (2001) stated there is uniqueness to this method and style of student teacher discussion that is distinctive, characteristic and definitive to a specific set of circumstances. The importance to the profession of nursing is "alternatives that challenge the underlying assumptions...” (Ironside, 2004, p. 6) of the status quo.

The immersion programme model matched up well with the narrative tradition because it fostered a safe haven for individual and group expression of feelings, reflection, and an atmosphere for all to truly express what each perceived to be "going on" in "their" clinical experiences, social, and personal encounters. Each participant vocalised many feelings and the affective issues generated dialogue on topics such as cultural differences, women's roles, access to health care and the 
overwhelming impact of poverty. In meetings with my students, we continually discussed our daily nursing activities and feelings in our professional roles working with patients, nurses, midwives, doctors, and ancillary personnel (Swenson \& Sims, 2003). We reflected on the multiple meanings of patient and care provider relationships, socioeconomic status and health care provision, nursing roles and functions, students' feelings related to the immersion experience, and the contrast of lifestyles. We also discussed fundamental aspects of the health care settings and lived experiences outside of the hospital as we participated in the lives around us. The student-faculty exchanges "fit" with narrative pedagogy because of the interpretive discourse that was an essential ingredient of the immersion programme. Theory is translated into reality through taking students abroad.

All seven immersion programmes focused on the sharing of professional and social experiences of students and the professor, both singularly and collectively. As we reflected, we discussed, and experienced overwhelming feelings about our clinical experiences and our social interactions. "The personal self and the professional self are not separate. The two come from the same lived experience, the same culture and social plane” (Francis, 2004, p. 245) and must be explored as they are indelibly intertwined. The atmosphere at times became charged, was palpable, and was fraught with grief and either the feelings of powerlessness or the "high" that came with sheer joy at personal or professional exchanges, connections and/or interactions. Diekelmann (1993) stated "We need a nursing literature that is complex and rich in the dilemmas that form day-to-day nursing practice, a dialogue that includes the events of both breakdown and triumph” (p. 249). It is important to acknowledge the gamut of emotions and cognition in order to explore, reflect, learn and transform lives in the profession of nursing. Group meetings, without "boundaries", that provide a safe and secure environment is an optimum place to begin. Narrative pedagogy encourages an atmosphere for students and teachers to work together to think and reflect about the significance of student learning and how it will affect their practice (Diekelman, 2001). “Critical reflection is an interesting and powerful thing. It can send us on an investigation which can change our lives by leading us to further discoveries about ourselves and others” (Street, 1995, p. 109). 
Narrative pedagogy emanated from research using interpretive phenomenology” (Diekelmann, 2001, p. 53) and there are many schools of thought about phenomenology. Powers and Knapp (1995) contended, "It is unlikely that there will be a single unified approach to thinking about and doing phenomenology" (p. 123). The unifying thread is exploring the lived experiences of people (Powers and Knapp, 1995).

Another component of narrative pedagogy, hermeneutics, was also important in my research. Dilthey (1910/trans. 1985) talked of specific experiences, that actually fit into the greater system of life experiences, that are understood through reflection on its significance; "Lived experiences are related to each other like motifs in the andante of a symphony” (p. 227). Van Manen (1990) described the concept of hermeneutic phenomenology as "a philosophy of the personal, the individual, which we pursue against the background of and understanding of the evasive character of the logos of other, the whole, the communal or the social” (p. 7). The "lived experiences" of the students in the immersion programmes were incorporated into their lives as they pieced together similarities of other professional and personal life experiences, journeys, and feelings. Diekelmann (1993) stated "hermeneutic understanding leads to an inclusive position on what kinds of knowledge and understanding need to be in the [nursing] curriculum” (p. 249). The major categories, such as Having Blind Trust, Valuing Others, and Transforming Experiences exemplified compassion, humanity, caring, justice, and dignity.

Utilising the tradition of narrative pedagogy, a positive outcome of the international immersion programme was expression of feelings and emotional support between students and myself (Ambrose \& Bridge, 2005; Packard, 2004). This was important because the entire psycho/socio/cultural environment, including student roles and functions were different from their past nursing education experiences. Students were provided a safe place in order to compare and contrast their emerging roles, functions and feelings. At immersion sites we discussed the impact of daily life, professional practice and personal and group social interaction on each student. Perceptions about professional practice, personal lives and social 
interactions were changed and thus world view was broadened. This also brought about emancipation through changes wrought to individuals.

In summation, narrative pedagogy utilises multiple qualitative research techniques to capture social data that addresses the potential emancipatory effects of bringing to the fore the inherent dialectic(s) present. I am particularly attracted to narrative pedagogy as an approach to teaching/learning, and a way to reflect upon students' and teachers' interactions when they “publicly share and interpret stories of their lived experience” (Ironside, 2001, p. 80). It is comforting to know, learn, and put a "name tag" on a belief system that I have incorporated into my pedagogical lexicon for many years.

A parallel idea is experiential learning and its role in identifying the dialectic through directly "experiencing”. In this research the dialectic refers to polar opposites when such opposition was unexpected. The next section will discuss Kolb (1984) and Vygotsky’s (1926/trans. 1997) theories on experiential learning.

\section{Experiential learning}

Experiential learning aligned with the dialectic by providing hands on nursing practice. The four principles of Kolb’s (1984) theory of experiential learning is the "process whereby knowledge is constructed through the transformation of experience” (Carpio \& Majumdar, 1993, p. 6). It included using concrete experiences, reflective observations, abstract conceptualisations and active experimentation (Kolb, 1984). Students underwent experiential learning in their dayto-day experiences. Experiential learning identified and crystallised those bipolar themes that fall under the rubric of the dialectic. There was a steep learning curve as each student adapted to and clarified her/his roles and functions in foreign hospital units, mobile units, and in community health centres. There were new routines, new medications and new treatments to understand as the processes of carrying out nursing functions differed from that of their home institutions. The language(s) was unfamiliar as was the food, housing, transportation, play and work (Goodenough, 1964, 1981). Students' prior understanding and familiarity were challenged as they entered the world of people in other countries. 
Kolb (1984) stated simply that experiential learning is "learning from experience” (p. xi). It is the synthesis of the art and science of a profession, formal education and personal development. Kolb’s (1984) Experiential Learning is looked upon as the "primer" of expertise of principles of facilitating learning both theoretically and by "experiencing” and incorporating the affective, cognitive, and psychomotor domains. By exposing students to new environments, where they could fully participate, combined with theoretical knowledge, a growth process took place. As Kolb (1984) described, "learning is a continuous process grounded in experience (p. 27). Potgieter (2003) supported this model “as a particularly appropriate model for a humanistic, practice-oriented profession such as nursing” (p. 9) “... as it stimulates true reflection and action upon reality” (Bevis \& Murray, 1990, p. 328).

Vygotsky (1926/trans. 1997) went further by articulating experiential learning as stimulating human development by individuals' unique participation in the process. Experiential learning as knowledge "gained through direct encounter with a subject, person, or thing has an effect [positive] on self-concept” (Burnard, 1991, p. 21). Reference is repeatedly made to linking the reality of the daily clinical experiences of the student to learning (Burnard, 1993). In nursing experiential learning has "been widely advocated for the teaching and learning of interpersonal skills” (Burnard, 1993, p. 60) which is an integral part of the nursing role. "Reflective learning [also] emphasizes the self as the source of learning (Boyd \& Fales, 1983, p. 102).

The dialectic is invariably linked to experiential learning because exposure to views, feelings, and teachings are in direct contrast to prior knowledge and learning. The student is exposed to differences in every realm of life, which fosters thinking, reflection, and a broadening of life experiences. New views can then cause dis-ease, and questioning of prior learning and views, new visions develop, new thought processes, challenges, and questions which leads to the dialectic. The teacher is also very involved in this process (Carpio \& Majumdar, 1993). 


\section{Dialectic Analyses}

The transcripts revealed three major dialectics. The first category was recognising the "Have and the Have-Nots". The second category was "Being an Insider and an Outsider" and the last category was "A World Shrinking, a World Expanding”. Each will be briefly defined, then discussed from the perspective of the students as gleaned from the interviews.

\section{Have and Have-Nots}

The world today has an insidious non geographic divide that distinguishes the industrialised, technological nations from the non-industrialised, nontechnological, developing poor(er,est) nations. Names such as the third world, developing world, developing country and the "south” distinguish the rich countries from the poor. In the developing world, daily survival is a way of life for millions of families. Relationships take precedence over material wealth and possessions. Extended families function as a cohesive unit and, "I", is not as common as "we" as a frame of reference. Individuals look upon the family as a unit rather than being made up of many individuals. Life is economically marginal for many, as families try to physically sustain themselves with continuing dwindling economic resources.

The USA is characterised by extensive material acquisition. Success is equated with personal "wealth" and "affluence” recognised by possession of "things, objects, belongings”, and "goods”. There is continuing pursuit for more and greater possessions, a drive for status via materialism, and the need for immediate gratification. The axiom, that "time is money", means that pursuits should be focused, expedient, and acted upon quickly. The I takes centre stage and relationships diminish inversely with the increase in technology. For example there are the children who sit in front of televisions and computers for many hours daily, in a world of their own, solitary companionship. Sometimes called the overdeveloped world; the USA is diametrically opposite to that of the developing world.

This first dialectic of "Have and the Have-Nots" was illustrated by exemplars from several transcripts, among them an exemplar from Alice. The health care world that Alice initially had known in the USA was one that employed nearly every 
available technological aid. In the immersion programme, Alice became quickly and painfully aware of the lack of equipment and medications that directly affected patients' health promotion, maintenance and restoration.

I think back to the medical hospital where they shared so much with us that wasn't pretty. They showed us the ICU where they didn't have things such as IV pumps. Every time the patient needed IV fluids we had to get a re-sterilised needle to put into their vein; the IV fluid ran in by gravity, and then when it was done the needle came out. There's just so much equipment that we have at hand in the hospitals in the developed world.

In this country [reference to venue] there was very little access to health care or equipment due to devastating financial constraints related to generalised upheaval and massive governmental changes. In general, prices for basic commodities became increasingly more expensive. People did not have the earning power or salaries necessary to pay for even the basics such as food, rent, transportation, and clothes. Before this sweeping massive governmental change, health care was an entitlement. Nearly overnight, the financial responsibility for health care was shifted to individuals poorly suited to pay for even the most basic needs.

As a result of these changes patients brought their own needles, syringes and medication to hospital. Each person also had to bring her/his own eating utensils. Each was also responsible for washing their plates, cups and utensils. Food was provided but the quality was so poor that relatives would send supplemental packages of food whenever they could. Any patient that could ambulate took meals in a common dining room. Visiting was not permitted and there was one telephone available for 57 patients. To type blood, a small marked saucer was used with several drops of blood and an applied solution. The midwife or nurse would then observe for coagulation in order to correctly identify and ensure the patient's blood type in the event of a needed transfusion. Alice felt that she came from the land of the "haves", to the land of the "have-nots" where supplies were scarce and reused over and over. Gloves were autoclaved repeatedly until they fell apart. Another example was the nurses using the same "sterile" instruments for wound packing and dressings on more than one patient. Alice worked in a country where the nurses' 
functions routinely included cutting apart gauze, packaging it into small packets and then hoping it got sterilised. Sometimes, it didn't.

Alice summed up her experience with yet another dialectic about the "Have and Have-Nots” and expressed it the following way:

I'd sum up to say that we're on this planet and we're not isolated. I live in my little community, but what I do has an impact somewhere else. When I think about conditions, lives and health care around the world, the experience I'm having is, as a nurse in the United States is not the experience of most nurses around the world. When I think back to the treatment room in the hospital with five or six patients simultaneously having invasive procedures without analgesia or aseptic technique, of all the labouring and delivering women I worked with, analgesia was not part of the process. I remember the burn patient who endured a lot of pain and suffering and then died because there were no medications except some creams that his family was sent to buy in a local pharmacy. There wasn't any hot water or soap for the nurses to wash their hands and there was not any toilet paper supplied anywhere in the hospital.

Alice was astounded with the inequities of resources, those seemingly available only to the "privileged" people of the developed world. She talked about the excessive use of resources in the technological world such as “one use” dressing sets, suture removal trays, throw away bedpans, and patient bathing equipment. She also discussed her accident of "geographic birth" into a developed world family and how this has totally affected who she is, her expectations, and her realisation about life. She felt that she was born into the world of the privileged and took for granted the amenities in life common to people in the developed world, also, that she would always be respected, have perceived control as a health care consumer, and lastly that she would receive timely financial remuneration for her professional employment.

Gigi was informative about the "Have and Have-Nots" and described, in her words, the existence of economic opposites.

I remember, riding the nursing school bus. Oh my god! We went way up in the mountains to really remote villages where people live in little huts, with no plumbing whatsoever. I remember the kids running around, no clothes, nothing and just looking at us and I remember when I came back I reflected, looked at pictures and it's almost as if you don't see it while you're there but when you look at the pictures you're like oh my goodness, look at us. We had on a 
white shirt and blue pants and a watch and perhaps some jewellery. Our hair was combed and we had makeup on, similar to the nursing students we were working with from the host university' nursing programme. Then you'd see someone else in the picture and they were wearing a pair of shorts, nothing, or a small wrap. What they had on was what they owned, their only possession, yet, they were always so happy to see us. I vividly remember that.

Another "Have and Have-Nots" issue for Gigi was the differential economic effects on poor childbearing women. Poor childbearing women, by necessity, used government hospital facilities if they lived in an urban area. If they resided in rural areas they would, perhaps have a traditional birth attendant (TBA) for their labour and delivery, having no other maternity care. These economic effects were compounded by other intervening variables such as illiteracy and powerlessness that accompanied poor women’s socioeconomic status. Gigi described her feelings about hospital maternity units as,

Two or three women in the same room giving birth and afterwards sharing a post natal bed. I remember my own labour and delivery experience in the USA; you were in your own room. Those experiences really impacted me.

The students' experiences in labour and delivery were always profound because of their own perceptions about working with clients and treating them with esteem, caring and consideration. It was difficult for the participants to witness such things as verbal and physical abuse, and many students had to emotionally separate themselves from the incidents when they occurred, in order to prevent their own emotions from overwhelming them. For poor childbearing women in the world of the have-nots, courtesy, dignity and justice did not exist. For students, anger and their own impotence were themes they felt compelled to address.

In setting the scene, taken from the interviews, for women of poverty, economics had a great impact on childbearing, especially for families that lived in cities and towns. In many rural and remote areas, the local traditional birth attendant offered intrapartal care that took place in the home of the parturient. Women using government hospital facilities for parturition often however had to share a bed with other patients. Linen, if not brought in by the client, was non-existent. Women in many of these societies are socialised to be demure and diffident, and to reveal little 
of their bodies or their lives. Hospital experiences, unclothed with an assortment of personnel wandering in and out, had many women trying to hide their faces because they were embarrassed. The women were told what to do and if perceived to not immediately respond, many were abused verbally and/or physically. This is the life of the have-not childbearing woman in the developing world.

Jasmine was also aware of the extremes of the "Have and Have-Nots" and the culture shock that accompanied her return to the developed world:

My prior knowledge was the media and the pages of history. To be able to go there and live, not as tourists, as we were not just passing by kind of sticking to the things that people recognise as highlights; we were in it. We were in their everyday life. Right there in their shoes, I mean as much as anyone can be. You really get an idea of what their day to day life is all about, doing the laundry by hand, hanging it to dry, the broken roads, the power outages everyday, the rationing of water - just all the conveniences here that we take for granted that they don't have.

It makes me feel really bad because we have so much over here and it just defines the extremes between the rich and the poor countries, a huge divide. It made me feel really kind of excessive about all that we have and take for granted.

It made me really see things in a different light than before. After leaving I went directly to Germany to reunite with 'my family' [Jasmine had been an exchange student for a short period of time when she was a secondary school student] in Germany. There was so much money there and everybody's talking on their cell phones. I was just really shocked. It was like coming out of a time warp and you pop out into the 'rich world'. I was really just blown away.

For Joan the dialectic of the "Have and Have-Nots" focused on the intrapartal process, specifically labour and delivery. In the country in which Joan participated in the immersion programme, although the hospital personnel were clearly in control, the expectations were that birth would have a positive outcome without the "benefit" of technological support. This was a country that was in a more fortunate economic position than many of the other countries. For this student sheer joy, could be experienced.

One of the things that was so remarkable to me is that women would get admitted, they would labour and their babies would come 
out and it was so uncomplicated. Here in the USA it is this big production with all of the different monitors and gadgets and everything beeping and the mechanical beds. In few of the women had IVs, most of them didn't. Foetal heart tones were auscultated intermittently using a Pinard stethoscope.

It opened my mind to other possibilities experiencing something so different from the rest of your experiences. If I had never had that experience and only worked in labour and delivery in one hospital I might be of the impression that this, the American [USA] way, is the only way that labour and delivery is done, period. But having been across the world, I saw the exact same processes taking place in a completely different atmosphere and questioned myself: ' Do we really have to do it that way? Is that really necessary? Do we have to use a foetal monitor for every single labour? Does that really do anything? Do we really need all the interventions and technology that we employ?' Since I experienced working in a completely different environment, it left the door open for me to change.

For Kaitlyn, the "Have and Have-Nots” was represented by the deprived socio/economic status of the indigenous peoples. This was a population greatly affected by absolute poverty. The absolute lack of potable water, sewage, adequate housing, and food was rampant, plus the people were socially ostracised. Daily existence was a hardship and people were heavily burdened.

The people in this town, as in most third world countries, really work hard. The odds are not stacked in their favour in terms of health and well being. They deal with incredibly dirty environments and incredibly cramped environments. Dirty water is contaminated by human faeces, worms and other parasites. Work, especially for the indigenous people whose daily existence is so extreme and so difficult, that to be told that they need to boil all of their water for human consumption, even for washing dishes, is ludicrous. It adds to the workload, burden and finances of the household. It is mainly the women who are affected and who already have so much of a burden. They try to keep things clean, they raise their children, many also work outside their homes, plus care for the small family plot for growing food for family consumption.

The indigenous people needed to have large families because there are more people to work. The more people that there are to work the better their chances of survival. The children were amazing. They were treated by the majority population, almost like animals; they were also continually talked about and addressed in a negative manner. 
Witnessing the rampant poverty of "minority" people on a daily basis both in and out of the health care system truly impacted students. It opened each to another world, and raised their collective consciousness.

\section{Being an Insider and an Outsider}

The second dialectic category was "Being an Insider and an Outsider" at the same time. Powdermaker (1966) stated “To understand a society, the anthropologist has traditionally immersed himself [herself] in it...” (p. 207) while always remembering he/she is from another culture. Students were constantly exposed to a dual view as they worked as members of the health care team and socialised with people. The participants were always conscious of the fact that they were from "another world" due to the newness and differences that they were constantly exposed to and participated in. The participants were afforded multiple "views" into lived professional and social lives of the people of the host community. This meant that although each student was immersed in the health care system and the social system this exposure offered visions of lives in stark contrast to the students' lives in their home community. At once they were participants, insiders, in their roles and functions, yet were also outsiders, as each could contrast their current lives with the lives they left behind in the USA. An example was a perception by the students of the lack of autonomy of the nurses in the host countries compared to the nurses in their home country. Another example was the lives of women and how their roles and functions contrasted significantly with the female students.

Being immersed in the culture and the health care system afforded a broad based view from which students, "insiders" in the sense that they worked as health care providers and were thus privy to the internal workings of the institution as well as becoming "extended family members" to the people with whom they lived and shared a social life. As “outsiders” they were products of their own culture and experience and could never fully identify with the world of the other person. Students' views were broadened by both perspectives, and in a complex way via exposure to the modus operandi of others, as well as the knowledge of their own practices in professional and social realms. Through being immersed in the health 
care and social systems of developing world countries students were constantly and acutely aware of the existing professional and social duality of the USA and the host country.

Alice described it in this way. She talked about her sadness about the locals' lack of awareness of their surroundings in their town [where we, the students and myself] also worked and lived. For Alice the dialectic was that hundreds of children in this community were living in orphanages and paediatric hospitals. The conundrum was that the local community was unaware of this phenomenon although this was a common problem across the depth and breadth of this nation. This situation provoked deep reflection and introspection for Alice. The dilemma was: how could the existence of the orphanages not be common knowledge? The issue of a "lost generation” was known to all in this country, yet, it was not really known: infants, toddlers abandoned in parks and streets by parent(s) who could not care for their offspring, where drug and/or alcohol addiction caused premature death. Perhaps the locals just didn't want to know. As an insider Alice had an intimate working knowledge of the orphanage, and the paedatric unit housing orphans when even those in the culture didn't know about their existence. As an outsider Alice believed all countries have systems in place to care for children who are orphaned and/or have parent(s) who are unable to care for them.

The centrally located orphanage where we lived during our seven week stay was completely unknown to our translators; they had never been there and did not know of its existence. THEY JUST DIDN'T KNOW that the orphans were there; they were shocked and upset to learn that these children were living without families in a community setting 1.5 kilometres away from their own homes. It made me feel like what's the secrecy here, why don't they know what's going on in their own community.

Another common dialectic encountered by Alice was as an insider in the health care system, versus the translators being the outsiders as they were university students majoring in English (two were mature returning students in their 40's, and the others were mid 20's). Working with us, the translators were directly confronted with the everyday workings of the hospitals, the personnel, and the equipment or lack of it. They were exposed to experiences that the average citizen would not have knowledge about. Students talked about this in the interviews. Although the 
translators were educated residents of this city, insiders, they were still outsiders, they didn't see, they didn't know the health care system. Their collective lack of knowledge and shock with prevailing hospital conditions and status of the employees was unnerving to them. They had no idea that nurses' salaries were chronically in arrears of three to four months because there simply was no funding available.

For Alice it was overwhelming, in the sense of her being an insider and thinking that all the information that she had privy to, was common knowledge of the population, which it wasn't. As an outsider it was difficult for her to understand that local residents had little or no information about hospital operating conditions. For both immersion participants and translators the hospital experience was profound.

It was a very eye opening experience for them as well [as us] and you know maybe one eye that they didn't want to open but they had to because they were there with us and they saw things on a daily basis that they wouldn't otherwise see.

For Bugs "Being an Insider and an Outsider” focused on the blending of being a novice traveler (outsider), and her exposure to "another world” (insider). She was, at once, privy to social and professional contrasts as an outsider and participant as an insider. Although she had worked with patients with different life experiences, she had never been immersed into a culture of differentness. She said that she emerged out of her little bubble world view, which was USA middle class, and realized that there are many ways to live a life. She had a preconceived way of order of how things should be which was USA based; in she was completely out of her element. Bugs felt she became, and was regarded as an insider, as she partook in the life around her through her work role and support functions in the hospital as well as personal friendships. As an outsider she was profoundly affected by her prior understanding of what she perceived to be universal health care or "life" in general. She perceived that the world's people all lived as she did and shared the same values and standards. These differences were a revelation for this student. As an outsider she was able to view this immersion experience of cultural differentness and use it to broaden her world view. Bugs found that her perceptions related to people's lives 
changed as she became accustomed to the "newness" that surrounded her. She also recognized and acknowledged that her understanding of other people was expanded and intensified. Her comfort level, professionally and socially, with people with diverse views and values steadily grew. By being immersed, Bugs came to a radically different understanding of what life was and meant.

We spent the semester learning about and the institutions of education, government, family, religion and economics. We talked about the social and health care settings that we would find. We looked at slides from previous trips and yet, it was just so different to discuss the issues on an academic level versus experiencing the real thing. The academia just doesn't speak as loudly as, you know, step off the aeroplane and smell the air and feel the humidity and look around and see just how different it is. I had never been anywhere. I grew up in middle class America and certainly lived in my own little clean bubble. I lived in this kind of false sense of global reality. It was really very beneficial for me to have this experience and to emerge out of my little bubble and realise that most of the world lives differently than what you know and think to be a consistent standard of cleanliness and orderliness. Immersing myself as a health care provider and socially participating in the culture around me made me reflect about my perceptions of lived lives.

Certainly the people we encountered lived very differently than what I was used to and they all managed to survive, and they appeared happy and they lived their lives and had families and relationships and children, and everything goes on just fine. It was a good educational, life changing, experience for me that was really beneficial, to be exposed to life outside of my little bubble. Just to get used to that, come to terms with it and be comfortable was the most positive thing that I could take away from the entire experience.

For Bugs, partaking in the lives of others made her realise that "we", culturally, are only able to "meet" on common ground; this was the sharing of professional lives and the socialisation process that we participated in. It is impossible to thoroughly understand the ways of others. People can only share enough of themselves to jointly participate in life experiences. Thus the outsider can never really "see" what life is like for another just as insiders cannot fathom the outsider world.

For Gigi “Being an Insider and an Outsider” was an intense and overwhelming experience when visiting patients with affective disorders. The 
psychiatric unit was part of the general (government) hospital and she was privileged to observe this hospital unit simultaneously as something of both an insider/outsider. As an outsider, Gigi was consumed by deep-seated instinctive emotions as she endeavoured to "look in" on patients, trying to understand her repulsiveness while still wanting to have care and concern for them. By being an insider, working on the maternity unit, and being oriented to the entire hospital, she was privy to all hospital units. She was made aware of a “world” she didn’t know existed. This is what I wrote about the incident.

$$
\text { Mental Hospital ___ [name of city]. }
$$

The psychiatric pavilion housed 35 patients. This is our first day, and as part of our orientation to the entire facility, my students and I are taken to the 'mental health' unit of this large hospital. For me this was the most profound experience that I have ever undergone while working in the developing world. For my students, an experience and a remembrance that will forever be imprinted in their living memory, perhaps even a nightmare. Immediately I think of $19^{\text {th }}$ century, Bedlam (in England) and other places of incarceration for the mentally ill.

We are led through a small courtyard, which separates the main hospital from this unit, into a dark, dank building. Our eyes adjust, and what do we see, naked human beings in small barred cells, and in larger group cells. Some inmates sit on the wet floor others are standing on their beds, sans linen, in their own worlds dialoguing to themselves; a few fight with each other. The more violent patients are in single cells to keep them from harming the other patients.

Most of these patients have been abandoned by their families because of the shame attached to affective illnesses. The families are no longer able to cope with the aberrant behaviours that are a threat both to the 'sick' member and to all the other family members. There is one particularly violent young man, housed alone, whose father makes a journey to visit him fortnightly; the staff are afraid of him, as he wildly gesticulates and screams and adds to the general melee of all the other patients. This man has been in this cell for five years living in his own mental hell; there are no medications to be given; the family cannot afford the cost. His bath is a few buckets full of water that are splashed on him every few days; his food passed through the bars, his bodily wastes hosed out daily. 
One of my students turns her back to the bars in order to listen to the commentary. She is too close, and one of the inmates grabs her hair and slams her against the bars. With the help of personnel we are able to extricate her, and she immediately comments about her behaviours and lack of attentiveness to her surroundings; perhaps it is the shock that has numbed her senses in this setting.

Gigi explored an insider's role as hospital personnel giving routine treatment to the mentally ill. As outsiders there was deep shock because treatment and housing of patients in psychiatric units, in the developed world, is diametrically opposite to the zoo like environment that was viewed. Gigi describes the scene as follows:

The psychiatric unit was absolutely mind boggling. It was like a zoo. It was worse than a zoo. It was worse than any zoo I've ever been to. I remember the cages, the bars, the cement floors and I remember it was all wet. The floors were wet and they were hosing the cages down to remove excrement. The entire place was foul smelling and it was just filthy.

I remembering being there and I felt so weird. The patients had nothing on, they were stark naked. They had no clothes and they had no possessions. It's hard to put into words when you see something like that, you don't even know what to think. I just had nothing to relate it to, absolutely nothing. I've never seen anything like that. You know the closest thing is feeling overwhelming sadness.

For Gigi, as an outsider, her view was one of consternation, shock, and sadness at the plight of the patients. As an insider she was part of the hospital working force, getting to know each patient and each unit where health care was provided.

In a similar way Trae experienced Being an Insider and an Outsider with a Roman Catholic priest who appeared to transcend the boundaries of his religious life. Trae acted as an insider, as part of the health care team and as an outsider, she witnessed a Roman Catholic priest directly contravene church doctrine regarding contraception.

is a very Catholic country. I was extremely impressed with the action of the priest. This was the woman's $19^{\text {th }}$ pregnancy; she was para 12. This was not her first experience with severe preeclampsia and not the first time that she had nearly died. It was a miracle that she lived, and the baby came out beautifully and that was a miracle too. This all occurred in the same place where we experienced a neonatal demise exactly one week earlier. This gave 
me a sense of hope, new direction and a real sense of the cycle of life and death and birth and death and rebirth.

The action by the priest really impressed me. He said that he would pay for the woman's husband to have a vasectomy because he knew that if she became pregnant again her chances of dying would be astronomical. Chances are that neither the mother nor another baby would survive. It made me feel like I have greater affinity for the Catholic Church even though I'm not Catholic. I now have the sense that even though Rome might be in an ivory tower, out of touch with what was really happening in the world, this priest and people like him have a larger sense of what is right for a human being. I think for that woman to have to go through another pregnancy would have been disastrous.

I feel an expanded sense of compassion. I see the cycle of birth and death within life. The impact of the baby's miraculous, healthy birth [after previously not being able to identify foetal heart tones and believing he was probably dead] gave me hope for a better world. This experience just made me feel good about humanity. That the priest would feel the way he did surprised me. I am happy that he would want to do something for this woman that he believed would be right for her, even though the procedure was in direct conflict with church doctrine. This experience has had an influence on my thinking and my practice.

It's prepared me very well. I feel that recognizing the positive effect that a person, as a teacher, as a nurse, as a member of a religious order can have on somebody's life is very empowering. It empowered me and also enabled me to make changes in my professional practice.

Observing and acting upon this decision of the Roman Catholic priest changed the student's practice in realising how empowering the role of one trusted person can be. It can greatly affect the life(ves) of those so entrusted. As an outsider, the student stood back with the realisation that she was observing a breach of sacred vows. As an insider, she knew this financially impoverished couple already had 12 living children, 19 pregnancies in all. They could not handle more.

\section{A World Shrinking, a World Expanding}

The third and last dialectic was "A World Shrinking, a World Expanding”. With globalisation the physiologic world seems to "shrink". Few in preceding 
generations ever encountered the endless possibilities for "expansion" of psycho/socio/cultural growth that abounds in cross-cultural exploration today. In my view, the necessity to expand one's world view is really a moral imperative. To me, morals embody the principles of individual integrity and conduct in the "practice of life”. Morals are the implementation of my upbringing as I daily interact with people and treat each with respect and dignity. As one traverses countries, cultures, religions, and ethnicities, the moral imperative of humanness, understanding and justice loom large.

As the potential exists for health professionals to practice in many countries during one's career there is the ever present issue of disparity that may arise. Individuals will be confronted everyday by unique experiences as each enters and participates in the world of others. Through exposure and immersion, one's world view can change, and the dialectic emerges as one encounters differences. For the participants in the programme, their immersion and involvement in the professional and social systems of the host countries caused students to question their prior beliefs, values, and knowledge.

...there are different degrees of comprehension and mindfulness regarding becoming aware of one's thoughts. In adulthood, knowing how you know involves awareness of the context, ... of your interpretations and beliefs and those of others...informed decisions require not only awareness of the source and context of our knowledge, values, and feelings but also critical reflection on the validity of their assumptions or premises (Mezirow, 2000, p. 7).

Participants were constantly “assaulted” with newness, and reflected and expressed feelings and ideas that were new to each person. Each had to make her/his unique decision(s) predicated on who they were as an individual and as a thinking adult. Being exposed to duality, such as different ways of problem solving, different ways of practicing nursing, and different life styles promoted the students' questioning and reflection. This, in turn led to new ways of viewing life because "life is a matter of becoming, a process of continual self-transformation and transcendence” (Kothian, 1994, p. 258).

At the end of each programme students had a much clearer understanding of who they were and thus were able to have the perceived control for reflection and 
introspection. They were able to interpret the action of opposing social forces. They were able to experience the tensions, contradictions, and the paradoxes utilising the concepts of duality in their professional practice and personal lives. There were affective changes that took place at the time of the immersion experience and also in the years following in both professional and personal realms. Expanding world view was manifested by immersion into a world where there was there was no indoor plumbing nor potable drinking water, or water for bathing. Water for cooking was collected in large metal drums when it rained. Students realised that people's lives were directly impacted by the vagaries of nature in countries where technologic status for health promotion and maintenance and creature comforts were barely existent. Other examples were bathing and washing dishes in the river. When it rained this couldn't be done due to all the flotsam and jetsam entering the river from the banks. Students were exposed to patients who were unable to afford the purchase of common medications and thus could not receive treatment. Students experienced maternal, neonatal, and infant morbidity and mortality as a fact of everyday life. Students worked directly with clients who were discriminated against because of their gender, colour, religion/beliefs, or country of origin. Students routinely encountered health care provision without technology and equipment, with outcomes that were sometimes the same and sometimes different from the USA.

The physiologic discomforts and inconveniences experienced by the participants were not the issues they focused upon in the interviews. Their foci were relationships, communication, openness, creativity, and understanding people’s modus operandi in relationship to their psycho/socio/physical environment. Emotions, attitudes and feelings were piqued. Rinne (1987) felt that the art of nursing should have equal time with the science of nursing because "human behaviour reflects our knowledge of, skill with, and attitude toward others” (p. 41). For the participants, there came the realisation of how nursing care is profoundly affected by the communication style of the care provider.

An important manifestation of, “A World Shrinking, a World Expanding”, was significant personal transformation. This was a process by which individuals' “take-for-granted” (Mezirow, 2000, p. 7) knowledge, such as value and belief 
systems, and a person’s “frame of reference”, (Mezirow, 2000, p. 7) was

fundamentally “challenged”. This enabled the individual to expand and grow. This transformation took place because of the professional and social components of the immersion programmes and the exposure to "new ways" of thinking and doing things. Freire (1972) called this personal liberation.

For Joan, “A World Shrinking, a World Expanding”, was directed at the ethnocentric thinking of the medical establishment of the USA and its practices as compared to other countries. She perceived that although "we" think we are "best", an attitude of superiority is not warranted. Joan felt that by immersing herself, as a health care provider in another country, specifically with childbearing women, she would be exposed to other cultural medical and nursing views that challenged previous held perceptions. Prior to this Joan had only worked as a health care provider in her home country.

In the United States we're so ethnocentric. We think we're the medical leaders of the entire world but the truth is our infant mortality rate is high considering how industrialised we are. We don't know everything and we don't do everything right here. I think that it's really important to be able to see how they do things in different places and to be able to take that information and use it to add to our practice.

The dialectic was reinforced by Joan's exposure to other professional practices and her subsequent reflecting upon the stance of her own country in relationship to their beliefs about the medical practices of practitioners in other countries. The world contracts with feelings of righteousness and superiority, and it expands when one abandons cultural imperialism.

For Lee, “A World Shrinking, a World Expanding”, focused on events encountered at the venue and how it caused shrinking and expansion of his world view. Racism causes the world to contract because dignity, respect, and humanity are negated by denying people basic human rights. Lee was surprised to find racism that was blatant outside his own country. As an insider in his professional practice, at the hospital in he was exposed daily to people being treated differently depending on their status in the given society. As an outsider these daily events were a “dawning” for him as his outlook became more global and he looked beyond the 
racism that was practised. He learned that racism is "alive and well” and doesn't always relate to the colour of one's skin. Racism causes divisiveness and causes the world to contract while understanding and tolerance promote expansion, via human growth. Through this immersion experience he was able to make personal affective changes as his world expanded in his professional practice such as validating the individual's worth beyond the realm of colour, ethnic identity, country of origin and religious practices.

Lee discussed the refugees and their social status in their country of "refuge". This implied not being employable, living in delegated quarters, not being able to apply for citizenship, and the fear related to being sent back to a war zone and a home and society that no longer existed as it once had. Many of the refugees had been living in for more than six years and were forbidden to work. Many of the refugee children had been born in but were not permitted to become citizens. At the time we were leaving large groups of these refugees were being forcefully repatriated to their ravaged home country. Their farms, places of business, and families had been decimated. They had no viable means to be able to support themselves. This is how Lee described his feelings,

There is racism in the USA and I thought going over there I wouldn't find as much racism because everybody was basically the same colour. Here racism is usually based on colour, there it was based more on religion and refugee status. I guess people have to feel that they're better than somebody so they have to put someone down. The refugees weren't allowed to work and were treated and portrayed as being inferior: intellectually, educationally, physically, the whole lot, everything.

From a nursing point I came away with the feeling that there are biases everywhere. When the refugees came to the hospital, if they were in serious condition they would be treated, if they came in and weren't seriously ill it seemed as if their care would get put on a back burner.

I felt like I was in the USA, almost, just for the racism and the biases and it made me a stronger advocate for patients. I saw what they were doing and I compared it to the biases in my own country. It was like a spiritual awakening for me; that we're all individuals, not just a religion or a race. It made me more confident in my nursing practice and interactions with people. I realised that assumptions are 
made based on your religion, race or colour. I go into a situation now totally accepting.

Lee's world view expanded as he reflected and was able to transform his prior views about racism by becoming more inclusive. Through his experience in the immersion programme, upon return to the USA, differences became more dramatic. The effects of his experiential learning changed his nursing practice in the USA to make him more confident in his nursing role. By being an insider in he was exposed to professional and social racism, by being an outsider he was taken aback by his daily experiences, and realized that many of the insular practices also are perpetrated in the USA. His world could have remained contracted but through exposure and immersion he experienced a great expansion.

For Natividad, “A World Shrinking, a World Expanding”, focused on ethnocentrism. Through the immersion programme she was exposed to experiential learning where opposing views, feelings and teachings about nursing practice, technology, medications, social relationships, materialism were part of her clinical and social experiences.

Americans [USA] consider what they do to be the benchmark because the evidence has been shown in a double blind clinical study and this is the gold standard for care. But, I have always believed in cultural context and doubted the true applicability of Western research to other cultures. For example, in the [name of city] Maternity Hospital, mothers didn't have babies on their breast in the first ten minutes after labour, and fathers weren't even allowed to visit while the mother and child were hospitalised [which could be from a week to three or four months if the mother had been considered a high risk pregnancy]. These are interventions that are performed as the benchmark, in the United States, to promote bonding and attachment at birth and this is thought to affect the remainder of a child's life and his or her relationships. But in what I witnessed in [name of city] families, parents and their children were just as affectionate and engaged, if not more so, than those I see in Western culture, never considering that there would have been any other outcome regardless of not meeting the standards of the USA.

The immersion experience did show me more clearly than ever that Western society is all about the 'one way' and that's what people equate as the best way or the only way. We do all this research to find out that this is the right way. But I think that there are many different 
ways that are equally valid. And that belief was validated during my immersion in

Throughout school [university], I didn't really find truth with what sociology or psychology classes would claim about human development. Stages of life are not universal for different cultures. I was usually appalled at how Asians and Pacific Islanders were depicted in textbooks, because these generalisations were mostly written from an ethnocentric USA perspective. I think that the immersion experience was just more validating than eye-opening to me because it strengthened my belief that it doesn't have to be one way.

Natividad had feelings of frustration related to what she considered a “superior United Statesian” attitude, an arrogance that abrogates equality, and she felt that was not a valid world view. The world, for her, contracted because of feelings of American (USA) superiority, and it expanded when equal validation was practiced among nations. For instance she felt that the USA dictated to other countries on how to provide health care that was based on cultural standards of the United States and that this was equated to cultural imperialism. She had difficulty with the USA's perceived position as being “the” truly knowledgeable nation versus "the rest". She felt vindicated by her participation in the immersion programme when she encountered a different set of operational principles that she felt were more flexible and functioned in harmony with what resources were available. Her belief that Western technological health care systems predicated their basis of operation on scientific studies that dictated the "only right way" to accomplish goals was further negated. "The psychoanalyst Alan Roland maintains that it is psychoanalytic imperialism to approach non-Western cultures as though the paradigms of the West are universal and ought to apply” (as cited in Ahmed,1988, p. 159).

For Nicole, “A World Shrinking, a World Expanding”, was centred on the relationships of the refugee population to that of the majority citizens (of the country). She was awed by the negative behaviours of the populace toward the refugee population versus how we were accepted and integrated into our professional and social roles. This dual recognition, of how two groups of human beings were treated so differently promoted both professional and personal life 
changes for Nicole. For Nicole the world contracted when inequality existed between the refugees and the majority population and it expanded when she was able to provide health care with dignity to all patients. Also, for Nicole, developing social relationships with the refugee population expanded her world. Professionally, Nicole talked about how she learned the importance of validating all individuals. This process of learning set in motion a very thoughtful and reflective time for Nicole. The following sequences describe Nicole's experiences.

We lived in this big tower block [built during the reign of the prior government] and there was a group of refugees that lived in the building directly across the court. We being foreigners didn't know who was local and who wasn't. There was always a group of people out in the courtyard and I just remember thinking these people are not accepted into the culture. I mean they are out there, they're families, each with many children, but all crammed into one room and they too were living in this boarding schoolhouse. They fled their own country because of war, arrive and live here so they wouldn't be bombed and killed, and yet are treated like outcasts while we were made so welcome.

really opened my eyes and taught me that whatever their last name is, whatever the religion, colour or ethnicity, it really doesn't matter. Who they were was not important to us as we didn't have the history. So we could just look at people and care for them as people and if the nurses or the doctors thought that person shouldn't be receiving care because they're a refugee, we just didn't share those same feelings. I felt that we should even treat them better because they were refugees. They've been through horrors that we couldn't even imagine.

It empowered me in that I can look at people as people and I can look at minority people and I can look at these people that are put down, on the lowest rung of the society, as people. My way of thinking came about after I left the country [clinical venue of immersion programme]. I've brought back with me, that I can communicate with people without speaking their language and that I need to set aside my preconceived ideas of a person because of their name or their colour and just look at them as human beings.

When I was there I was doing it but I don't think I thought about it because I didn't know, as I said, that they were not supposed to be treated that way and then coming back and thinking, and reflecting about it and writing in our journals and writing our paper [formal course assignment] and talking with the other students about it. Why weren't we treating those people the way the other people were, well 
we didn't know. For many of my fellow students the true changes didn't happen while we were there.

My memories of this trip keep coming to mind and it's still happening today, changes that I have made and still have to make. I think of those people [refugees] and hopefully they are being treated differently as I'm sure many still haven't been able to go home. Maybe it's gotten better and maybe it's not but I just think the biggest part of my change, awareness and who I am and how I work happened after I came home. I then was able to start putting that existing situation in context of how I live and for me the changes continue to happen.

We have to treat all people as worthwhile human beings, especially minorities because they get the brunt of all evils from people it seems. We need to treat everyone fairly but maybe we need to go out of way and treat minority people and people of poverty and whatever condition with respect and be aware of how we're treating people.

It was a wonderful experience, and it just really empowered me and my ability to care for people. I came to care for these people in a deeper way than I would care if I was just visiting a country because I was caring for their mothers and their brothers and their children. They trusted me because I was caring for their loved ones. What an incredible way to visit a country, to be able to care for people and for them to return such warm feelings towards us. I wouldn't change it but it has changed me.

Nicole discussed the immersion experience and how it changed her because of her greater understanding of life and the expansion of her world view. The dialectic for Nicole was her exposure to racism and her extreme discomfort with the way people who were not members of the majority population were treated, both in the health care system and as ordinary citizens. She spoke about her personal "clean slate” and how this affected her interactions with her neighbours [refugee families in ] and clients [those of the refugee population]. She was not of the "mind set” of the people of our host country, through her cultural innocence, and was able to cross a cultural chasm and form a connective bridge of understanding that has had major continuing effects upon her life. The world contracted with mistreatment of the refugees, but for Nicole the world expanded as she became empowered with her knowledge about racism and its insidious effects on people. She also felt empowered 
through the realisation that she could communicate without speaking the language; it would have remained contracted had not she "taken risks" to grow. Her world expansion was totally summed up with her statement of “I wouldn't change it (venue), but it has changed me”. During the interview Nicole stated that in her personal life, she goes out of her way to help people who are both different and in need. She also says in her professional life, that she tries to support each patient's persona and diversity. Nicole’s world expansion has continued to evolve and reshape her life.

\section{Conclusion}

Students' awareness of the vast differences in the way of life of people in the developing world versus the over-developed world was a revelation to many. By living professionally and socially in this other world, students became empowered through their newly found awareness of other parts of the world and other people. This brought about emancipation through the realisation of new understandings, options, and the abilities to act. Students were able to make changes in their own lives. The dialectic was a constant as students' exposure to duality continued on a daily basis. The students became aware of a plethora of newness, in human relations, in daily living, in their professional and social lives. Through deep reflection, expression of feelings, and discussions about daily experiences deeper meanings were brought forth which enabled disparities to surface, feelings to coalesce, and personal changes to take place.

For students it was exposure and awareness that brought about change. Enlightenment "is knowledge of self in relation to the world...” (Powers \& Knapp, 1995, p. 34). A "light bulb” was turned on when students talked about such things as the powerlessness of patients. The students' realisation of their client interactions through direct health care provision engendered feelings of empowerment. Empowerment "involves social transformation through some form of educative process” (Powers \& Knapp, 1995, p. 35). Empowerment was brought about by students' individual strengths in their abilities and students became "liberated" through "a state of broadened rationality and reflective clarity where people have a 
sense of themselves and can freely and collectively determine the directions they should take in life” (Powers \& Knapp, 1995, p.35).

In all the interviews, students talked about the ongoing, persistent life changing effects of the immersion programme in both their professional practice and personal lives. They talked about their subsequent flexibility and ability to be creative and innovative with each client. Students talked about knowing who they were and how it centred them in their provision of care to patients in the USA. They talked about perceiving each client as an individual with her/his unique set of needs, each of whom deserved absolute respect and understanding. Mezirow (2000) contends “A new way of seeing has to lead to some kind of action” (p. 335). Through the exposure and immersion the programme provided, the participants created newness and differentness in their work and home based lives. They discussed how some of their beliefs could no longer stand up to scrutiny. For example, students questioned the hegemony in the host country(ies) and the USA in terms of how it affects health care. Students focused on the disparities: those who could pay fees for services fared much better than those who could not; people who were perceived to be of minority status were treated differently than the mainstream patient; the powerlessness of many patients and their families; and the overall physical state of the hospitals. Students also talked about the safety net that exists for the poor in the USA versus none in the developing world. Some of the other issues were racism, ethnocentrism, quality of care, technologic nursing, caring, and establishing a real human-to-human connection. As mentioned previously the dialectic has been echoed in my own personal reflections. This second level of theme reinforcement adds depth to presented thesis concepts.

The following vignette captures many of the embedded concepts that epitomises the dialectic. I wrote this vignette many years ago and feel that it represents daily occurrences in the lives of poor childbearing families, daily situations that students encountered:

\section{Neonatal demise/Cord Stump Exsanguination}

A grandmother, her young teenage daughter and a grandson of twenty-two hours of age appear at the door to the hospital. The 
neonate looks moribund. They are shown to a paediatric cot, told to breast feed the baby, he who is barely breathing, with shallow breaths at 110 /minute. We take the baby to the oxygen tank, although I know that he is beyond this, but this is all that we have to offer. It is clearly labelled, we turn it on, and it is empty. Many bits and pieces lie around in this modern hospital built in an election year. There are doors with placards stating: X-ray, Lab, Switchboard, Toilets, Physical Therapy. Open all and they are empty rooms, 'empty promises', as the staff would say.

This is a family that has crossed the river in desperation, to seek help, as there is none on their side of the river. This is a different country, foreign language, different culture, and the old enmities of the two countries surface as the staff blame the family for the baby's condition; for being ignorant, for being stupid. The continuing racial tensions rise; it does not take much to inflame, as this is seen, and heard in daily comments by the populace when talking about or working with the people from the 'other side of the river'. This is not new to these two countries both mired in poverty, illness and hopelessness.

We are able to secure permission from the hospital authorities to take the neonate to a 'better equipped' hospital, about ninety minutes distance. I know that he will not survive. We get the jeep from the local priest, and, on this occasion, do not seek the permission from the local army officer, as this is a baby, and we are hoping for understanding at the military checkpoints. As we drive I get the story, the string securing the umbilical cord stump had loosened and the grandma tells me that the baby had a little blood coming from the 'belly button place'. We are on the road [euphemism] for forty-five minutes and the baby expires; we turn around and reverse our direction. On the way we stop and the family departs through the forest on their way to cross the river and return to their own country. The keening of the two women is heard for a long time. I bereft, my student, in more control, lament yet another neonatal demise; this baby who weighed 3.8 kilos, an unusually high birth weight for either country. This baby from whom I had to retrieve the sheet that enfolded him because it belonged to the hospital. This baby whose birth and death were never registered; as if he had never been in this world; family grief ritualised and stylised, through keening, yet keenly felt. As I write this vivid memories return as if it were yesterday. I [we] felt impotent at the vulnerability of women and babies.

Painfully I reflect on the "resources” that don't exist: the basics, simple to more complex technologies; the lack of compassion; the absence of 
promoting human dignity; the glaring omission of humanity, humanness, and justice; and the blatant racism. These elements add fuel to my compulsion to expose students to the true reality of life as lived by the majority of the inhabitants of this planet...not the "reality" as portrayed by the variety of television reality shows...but reality as it exists.

\section{Summary}

This chapter focused on the analysis of the three major dialectics of the participants. Changes that occurred for the students were addressed in the professional and personal realms as well as world view. Narrative pedagogy and experiential learning were addressed because they are foundational to the findings of this study. In the following chapter the thesis questions are answered, the evolving concept of culture is discussed, and how one socially positions oneself. I further reveal more of myself and who I am. 


\section{CHAPTER VII}

\section{CREATIVE SYNTHESIS}

"Being human profoundly means to be open to the world, a world, that is, which is replete with other beings to encounter and meanings to fulfill” (Frankl, 1969, p.

113).

\section{Introduction}

This chapter speaks to my thesis journey and the revelation of how students' experiences, stories, perceptions, and transformation complemented and affirmed my beliefs to continually challenge myself, and students, through international programmes consisting of total personal, professional, and social immersion. The two questions addressing the impact of the programme on the students, and how the students' experiences factored into my deeper understanding of myself will be addressed. At the initial onset of this project I had no realisation of the enormous contribution of the participants to my epiphany.

I have also looked at societal factors that influence the health status of poor childbearing women. I have included this because greater than 530,000 (Rosenfield \& Schwartz, 2005) women annually die directly related to their pregnancies. Ninetynine percent of these deaths take place in the developing world (Towards evidence, 2001; Germain, 2004; Graham \& Hussein, 2004; Tsu \& Shane, 2004), an environment which has been the major focus of the international immersion programmes. I also was clueless about participants’ personal and professional changes that were described as "life changing", which is also addressed. Culture, another very important component is affected by societal institutions and in turn the provision of health care and its cascading effects, upon individual patients, families, and minority peoples, are discussed in depth. The latter section of this chapter reveals more of who I am and answers the research question of why I return again and again to work with poor childbearing women in the developing world. The research questions that guided this research asked: 
What impels me to return again and again to work with poor childbearing women?

How did the international immersion programmes impact the personal and professional lives of student participants?

Can the student experiences help me to understand myself better?

The answers to the questions enabled me to gain further insight and knowledge as to who I am, and led me, ultimately, to my epiphany. In the 'international immersion programmes students practiced nursing as well as entered into the social life of the venue community. For nursing students who were raised in the USA and thrust into a "new world", daily living, public services and health care systems were diametrically different from what they had ever experienced at home and deeply affected each participant. For myself, experiencing other ways of being, have become part and parcel of my life, and for my students I just did not fathom the great changes that would transpire. Having the students with me enabled me to see “that” part of the world through their perspective. This provided more complete viewpoints of lived lives; provided insight into the meaning of the immersions; and provided words to describe what they saw, felt, and experienced, thus I was further propelled on my journey to my epiphany. The next sections will discuss students' perceptions of the programme and the impact on their personal and professional lives.

How did the international immersion programmes impact the personal and professional lives of student participants?

\section{Perceptual Changes}

The word perception is defined as "a single unified awareness derived from sensory processes while a stimulus is present” (Flexner, 1983, p. 1437). The American Heritage Dictionary of the English Language (Morris, 1976) defined it as “becoming aware of, in one’s mind; to achieve understanding of” (p. 972). Potter and Guzzetta (2000) defined perception as “a human response pattern involving the 
reception of information" (p. 320). Also, perception can be defined as "seen in totality/wholly seen (Morris, W. 1976, p. 972). There are multiple hues of interpretation and even significant variance in perception depending on who is doing the viewing. Wolf, in her foreword to the book Women in the Material World (D’Aluisio \& Menzel, 1996) succinctly states

All too often, the picture [perception] of the planet we carry around with us-a picture formed of our own experiences and the stream of information from the mass media-is at odds with what is real and important. The world is much more diverse, sorrowful, and splendid than the white-washed and overwhelmingly present stereotypes many of us have in our heads. (p. 9)

By total immersion, students moved beyond what they were used to and what they knew. They learned to distinguish and discriminate the finer nuances and segments of a culture by existing in it. Student participants realised that the scenario of "judging a book by its cover" was not in the least, applicable.

The journeys to developing countries broadened students overall perceptions professionally, socially, and personally because of the stark differences in lifestyles (Devereux, 1967; Mann, 1972). These disparities and dissimilarities strengthened and facilitated the process of altering their perceptions because of the realised differences. For students in the immersion programme, perceptions changed based on their experiences in developing countries.

Students' "lenses” were modified as they were challenged to live in circumstances that were "worlds apart" and "far removed" from their lives in the USA. The perceptual changes were categorised as follows: economic, education, family, government, and religion, the traditional sociological institutions of society. The cumulative affects of the society in which one lives has profound effects and/or consequences on individual citizens (Ahmed, Adams, Chowdhury, \& Bhuiya, 2000; Bouchet-Saulnier, 2002; Challenges for, 2001; Chapman \& Berggren, 2005; Levine, 1994, 2000a, 2000b, 2001; Levine et al. 2002; Livingston, 2004a, 2004b; Williams, 2002). The following vignette depicts a scenario that includes the five institutions of a society and its cumulative effects on a family. 


\section{Fulminating Pre-eclampsia/Grand Multipara}

A grand multiparous woman crosses the river from one culture to another, from one country to the neighboring, in search of urgent health care. She carries a short note from a French expatriate midwife: "Please help this woman; she is gravida 19 Para 14. Her $\mathrm{B} / \mathrm{P}$ is $230 / 180$ ”. She and her husband walk; the temperature is $37 \mathrm{C}$ with matching humidity, and the midday sun's rays burn the dry earth. For two hours with fulminating pre-eclampsia, she and her husband slowly wend their way to the hospital. Her abdomen is rigid and we are unable to hear any foetal heart tones. Her head aches, her eyes are photo sensitive. We must seek permission, urgent permission, from the officer at the local army barracks to move her to a better equipped hospital. This takes forty-five minutes; the ongoing enmity persists between the citizens of these two countries and it is with difficulty that we secure permission; perhaps because I, a foreigner, act as an intermediary it finally happens. Her husband is very solicitous and caring, cradling her head on his lap. We begin our journey and my student communicates to me that the woman needs to have a bowel movement. I pull over to the side of the road knowing that this birth is imminent. A live male infant with a lusty cry is delivered.

We happen to be stopped near a settlement of a few rudimentary houses, and when the women within hear what is happening one donates a covering for both mother and baby. We continue to the hospital and with reluctance the woman and her baby are accepted as patients. This is not the first time that this woman has had fulminating pre-eclampsia. I reflect and wonder will she make it through the next pregnancy?

The couple, who like many others in the developing world, continue to reproduce even though the family struggle to feed themselves. This is a couple who must seek medical assistance in another country as there is none available for them in their own. They are a religious couple, who despite utilising a natural family planning method continue to have another child every year or two. The couple's children do not have access to education. The entire extended family participate in subsistence farming in order to survive. I reflect and intuitively know that all I can do is palliative in nature, a Band-Aid to stem the current family crisis. I feel a sense of impotence which then reinforces the influence of societal institutions on students' perceptions and transformations. 


\section{The Influence of Economic Status}

The economic conditions in the developing world were, and are, quite different from the USA. America is the "land of plenty" while most nonindustrialised nations are poverty stricken. Poverty has multiple dimensions, psychosocial and economic. Poverty, in resource poor countries, includes “income poverty, hunger, disease, lack of adequate shelter and exclusion” (Sachs \& McArthur, 2005, p. 347). At the family level, "variables such as employment, income and ownership of consumer durable goods, type of drinking water and sanitation and housing” (Fotso \& Kuate-Defo, 2005, p. 207), and also the paucity and availability of health services truly impact each member of the family (Anand \& Bärnighausen, 2004; Mavalankar \& Rosenfield, 2005; Mehrota \& Jarrett, 2002; Wagaarachchi \& Fernando, 2002; Poverty is pronounced, 2001). Gender inequality, illiteracy, powerlessness and social injustice also relate to poverty status because each person cannot attain her/his full human potential (Grown, Gupta \& Pande, 2005; Unterhalter, 2005). Students perceived that poverty was pervasive at all but one of the venues used for the immersion programmes and impacted all aspects of the lives of local community members. Poverty was manifest in families, communities, housing, transportation, health care provision and health, food intake, etc. There was often inadequate water, food, housing, appropriate clothing for varying weather conditions or health care provision. But, when looking beyond the obvious "lack of" resources and government infrastructure, students perceived something more. They saw in the people, great delight as they lived out their lives. Students participated with families living their daily lives and saw happiness, togetherness and celebration. Students found the pervasiveness of poverty was never the focus of life. While sadness and tragedy touched their lives these issues were accepted as routine and people moved on with their lives. The grime, pollution, substandard living conditions, or dismal state of the hospitals was simply as the way things were. Students saw the joy of people's lives despite the hardships of daily living.

Students' perceptions were completely changed by stepping out of the comfort zone of their lives and experiencing the lives of others. Students 
experienced a rare gift in their acceptance as family members, participating in mundane daily living: household tasks, intimate conversations, marketing, and also family developmental milestones. In small communities local children were watched over by all families, and welcomed in any home. Students, too, assumed this role in their spare time. Students were initially surprised as local children would follow them around town, smiling and laughing. Students developed trust over and over again with the young of a community and often stopped to play games with them. Students even participated in the care of an orphaned neonate who belonged to the community as a whole, and enjoyed being invited to the landmark $15^{\text {th }}$ birthday party of a young ___ [name of country] girl along with all the families in the town. These experiences further expanded participants' affective development and acceptance of value systems new and different from their own. These experiences further added to their repertoire of life in its many guises.

Another area where perceptions were changed had to do with the economy of health care workers in the developing world. Economic deficits impinged on nurses' financial status and the nurses' abilities to support their families. In a few of the countries salaries were only paid sporadically, which meant that nurses largely worked for free. Students bonded with these nurses, worked with them, spent their social hours at their homes and saw their professional "labours of love”. Students marvelled at the nurses' dedication to their patients and to their profession, while each struggled mightily to provide the basic amenities for their own children and extended families.

Students worked in hospitals without technology and cared for patients with few available resources. Without technological equipment, they found themselves focused on building trusting relationships and offering the best physiologic care that they were able to provide in the prevailing environment. Participants learned to understand the humanness of each patient. They increasingly perceived that all humans share basic and complex needs, which always creates a bond of common ground. Participants went beyond language in communicating with their patients and realised their abilities to connect, convey and express feelings, on many occasions 
non-verbally, which was perfectly sufficient. In nursing in the developing world, work was not about economics.

Student perceptions were also seen in the more complex economic arena of host countries. While the lack of economic security has the biggest impact on women and children of poverty it does affect the whole family unit. The economic stability of a family is threatened when a pregnant woman can no longer fully perform her role and functions. Communities are further affected when children of a mother unable to execute her daily duties must be cared for by the extended family, other community members or the father. Students recognised working inequities as well. Women routinely have a “double day” (Meleis \& Lindgren, 2001; Royston \& Armstrong, 1987), sometimes called the "second or third shift” (Browner \& Leslie, 1996), where they complete household tasks, care for children, act as wage earners, and work the fields all in a day, everyday. The economic impact of deforestation, drought, and depleted soil commonly cause poor harvests which means little if any, excess of products that are available to sell in the local market. Students were surprised that there was seldom enough food to sustain the family. Women's time is greatly impinged upon by the hours necessary to collect firewood for cooking, water for irrigation, drinking, and washing clothes whilst still being expected to provide economically (Browner\& Leslie, 1996; Cheru, 1992; Craft, 1997; Haselgrave, 1997; Kendall \& Li, 2005, Levine, 2001).

Additional economic factors were seen as women worked outside the home and were paid less than men who were doing the same work. They usually worked for others and encountered wage discrimination and sexual predation as a fact of life (Self, 2005). Women worked long hours for little financial gain, and often under very harsh conditions. There was also unequal distribution of household tasks between male and female household members. Child care was considered the sole domain of women (Browner \& Leslie, 1996; Pick, Ross, \& Dada, 2002). Moser (1989) in her article "Gender Planning in the Third World: Meeting Practical and Strategic Gender Needs” discussed the significance of women’s roles as related to societal progress. Moser discussed women’s “triple roles”, as being “productive, reproductive, and community management” (p. 83). Only the productive role was 
viewed as vital, as it involved paid employment directly supporting the family. Students worked alongside women doing their household tasks: sweeping the earthen floors of their homes, washing the wooden walls, and sweeping the entryways. Students helped to collect water to wash dishes after meals, as well as shaping cookies in a household bakery, evenings, for women who were multitasking, who were trying to keep their families intact; these were some of the daily routines. Students readily participated in the daily chores of their families' lives which further promoted their relationships, the entwining of lives.

Another economic issue identified by students was that of the rural poor moving to the cities. Students witnessed even greater poverty among this group who were also isolated from their extended families. Students watched as women, accompanied by their small children carried bricks, upon their backs, for eight to ten hours per day. Just outside big cities students watched as entire families, with children as young as five, each family member, hammering rocks into small stones to be used for building roads. They viewed migrant communities on the edge of cities whose shelter consisted of cardboard that was secured daily by their children, from a rubbish heap, to provide night-time relief from the monsoon rains. The movement of the rural poor, into large metropolitan areas, has become a widespread phenomenon in the developing world as impoverished families flock to the cities with the hopes of increasing their economic viability. These workers are unskilled, usually illiterate, and the majority often leave behind the extended family. What they find is not what they envisioned: dire poverty, scarcity of employment, and psychological and social loneliness. Family units dissolve and women become heads of household desperate to find employment in order to feed themselves and their children (Islam \& Tahir, 2002; Levine, 1990; Moser, 1989; Moss, 2002; Epp \& Ringheim, 2004).

In Peru a name has been given to areas that develop on the fringes of large metropolitan areas where houses are quickly and poorly constructed; they are called “pueblos jovenes” which mean "new towns”. Often a derogatory meaning is associated with these immigrant towns. In these areas young children were left to their own devices for eight to twelve hours daily. The eldest child, perhaps seven or 
nine, in charge of all his/her younger siblings; this included meal preparation, supervision and safety. Serious injuries, even deaths were common-place as the terrain was mountainous. Women worked as street vendors and their babies, the good babies, the ones that slept all day, were placed in cardboard boxes under their stalls, so mum could conduct her business. Usually the woman was an employee, a paid labourer, who spent many hours daily attempting to earn enough money to feed her family. This was my experience and my students with childbearing women. Students could readily identify with all this as they bore witness to the injuries of babies and children, and shopped in market places similar to the ones described above.

In similar practices accompany economic pressure as women work in the fields alongside their husbands and other members of the extended family. Children too young to work are simply left at home without adult supervision. Children are responsible for each other's welfare, each learning his/her gender role.

\section{Education of Females}

The student groups were surprised when considering the institution of education. They thought education was universally available, free, and rigorous. In many countries in the developing world primary and secondary education is the financial responsibility of individual families. Students learned that school fees were imposed which included books, paper, pencils, and compulsory uniforms. For the majority of families this amounts to a considerable sum of money that few can afford (Germain, 2004). One-sixth of the population of the world without access to education are women, this was a revelation to students, as they began to fathom how profoundly women and girls were affected (Unterhalter, 2005). In many countries it is not considered necessary for girls to have formal schooling as this poses a financial burden on the family. Additionally, girl children usually leave their families of origin during early teen-age years, or sooner, to marry, thereby being absorbed into their husbands' extended families. Loyalties and obligations then belong to their "new” families (Dollar \& Gatti; 1999; Filmer \& Pritchett, 1998, Schultz, 2002; UNICEF, 1999). 
The education of female children has been shown to be important in promoting and maintaining child health. The more education a mother receives, the greater the decrease in the morbidity and mortality of children, even two to three years of formal education makes a difference (Barrera, 1987; Schultz, 2002). Fertility also declines with female education (Schultz, 2002). For example "In the southern Indian state of Kerala, where literacy is universal, the infant mortality rate is the lowest in the entire developing world-and the fertility rate is the lowest in India” (State of world's children, 2001, p. 8).

Schools, too, are often geographically inaccessible and directly conflict with family economic survival (Dollar \& Gatti, 1999; Schultz, 2002). Children often have to walk several kilometres to arrive at their schools where 50 or 60 children will be in each classroom; if desks and benches exist they may have to accommodate more children than they were originally built for, and children often come without provision of food or drink. When decisions are made about school attendance it is male children that have priority, as they will be the ones to support their parents in their old age as well as their wives and children. Girls rarely attend school, and if they do it is usually for two or three years.

For students coming from the developed world where education is compulsory, the thought, the idea, that the majority of people were illiterate/functionally illiterate was a remarkable experiential learning process that never occurred to them. Students' perceptions were tested in that the "world" they left behind was educationally opposite from the world that they were currently inhabiting. Students had 14, 15, 16 years of formal education while working in communities in which the people had a few years, or none, of formal education. Instead, life provided the educational skills necessary to survive, i.e. life skills not books. Students witnessed highly sustainable communities with only a few members who could read; literacy was not a factor in getting things done.

This insight of students reminded me of a book that I read many years ago, Touch the Earth (McLuhan, 1971). It was about mid $18^{\text {th }}$ century America and the English colonisers and the local Native Americans. Politicians offered to "educate” young Indians in higher institutes of education. When returning to their families and 
communities as "hunters, warriors or counselors" the young men were perceived as “totally good for nothing” (McLuhan, p. 80). Education must have relevance to the “culture” of one's lifestyle, thus all the "book" learning could not sustain the individuals in their home environment.

Students were always aware of young children that worked alongside their parents in the fields, sold home grown produce in the local markets, or just entertained themselves while their parents worked stalls. Participants often met young children going door-to-door selling fruit and/or vegetables, carried in baskets on their heads or backs; bread, cookies baked by their mothers and grandmothers also sold in the same manner. Girls of five, six, or seven, were at the community wells collecting water for cooking family meals and then returning to clean the dishes and pots and pans. It was not possible for these children to attend school; their labours were needed for family survival. The following vignette describes how families remain “stuck at the bottom of the heap”, generation to generation.

\section{Two Walls and a Ceiling}

The heat is oppressive; the fumes from cars and trucks fouling the air as all stand with engines running awaiting the signal to move. This is one of the main roads to the entrance of the capital city. We roll down the car windows, and people turn off their vehicle engines as the minutes stretch into a half hour and beyond. I get out of the car hoping to have a wee bit more circulating air and I notice movement in the rubbish tip which is about five metres directly in front of me. Two young girls are scavenging for any item that their families can possibly use; food, or materials. I watch intently for three quarters of an hour, traffic still at a standstill. Although I do not speak their language I indicate to them to come closer to the car. My students and I turn over our lunches to these young girls, and watch as they return to continue their search. They find a few pieces of cardboard and set off for home. They leave and the monkeys move in to begin their reconnaissance for sustenance. The traffic begins to slowly ebb forward and shortly we see the two girls laden with their lunches and cardboard arrive home. We are stopped once again and watch as the day's booty is used as a replacement for its sodden predecessor; two walls and a ceiling. The conditions they live in are appalling and the children, never having the opportunity to attend school, perpetuate this cycle of poverty, want and ignorance. We are all deeply affected by these everyday street scenes. 
I reflect on this scene often, children just like yours and mine, except that these millions of children do not have the basic everyday benefits of food, a roof over their heads, clothes, access to any education, and that they must work toward sustaining basic family survival. Students ruminated and found this unbelievable, yet they saw similar scenes everyday. They watched in awe as a child, many children, dressed in rags found a twig or stick, and knew happiness engaged in playing as children the world over do.

Students commented on breastfeeding as it was more obvious than in the USA and they were amazed at the ease with which their patients, and others, put their babies to the breast; young girls having grown up with female members of their extended families breastfeeding and thus lactation counsellors were non existent, breast feeding was the norm at the majority of the venues.

\section{Family: Women's Roles and Functions}

Students were energised by the cohesiveness of families yet expressed feelings of sadness related to women who had little or no status in their societies. Participants learned that women needed to ask permission of their husbands in order attend antenatal visits. Students who were used to handling their own financial affairs were shocked to discover that women had no access to any financial resources, having to ask the head of household each time a purchase may have been necessary. Students found relationships with women of the families was much the same as it would be in the USA; daily tasks, conversations about life and values, and sharing jokes were commonplace.

Participants were constantly aware of the trust that families developed with them, and simply because they were there they became part of the family and community life. Participants contrasted this with life in the developed world and the decreasing importance of relationships, the lack of trust and the distancing of people. Participants felt very comfortable with the social situation and support of "their families"; they shared their lives at home and pictures of their families, homes and pets. Host families always wanted to know all about the student and her/his family, their school and vocational plans, their family relationships, and their feelings and 
philosophy of life. Many of the families learned about nuclear families and aunts, uncles, cousins, and grandparents that lived thousands of kilometres away, and that were visited only one or two times annually. Host families were fascinated by stories that students shared, about their lifestyles, about the material items that they owned, about the poverty that existed in the USA. Many local families could not believe this, and thought that all citizens of the USA were "rich".

Student perceptions of family were challenged when it was noted that women sometimes abandoned their children in the marketplace; a parent relinquishing a two year old or a six year old because she could no longer afford to feed him/her. Students also observed that in order to feed the family, a common practice was to send young children to the central marketplace with plastic bags to pick up the discards thrown down to the banks of the river. These discards were used to feed their families that day. Children were also sent to local trash heaps to find usable materials for housing the family. Families lived on the edges of large cities because they left their rural home in hopes of finding work. It was common for family units to drift apart due to the multiple pressures of just trying to survive.

Within the family, a majority of women had no rights of inheritance (Grown et al., 2005). At the time of marriage, any property that a woman might have been entitled to was turned over to her husband (Agarwal, 1994). Women were unable to make any decisions without first consulting with their husbands and/or mothers-inlaw. They had to honour their husbands and be at their beck and call. Women have been beaten, even murdered, due to actual or perceived infractions of the social code, by the male head of household (Holm, 2001). These crimes often go unpunished (Fikree et al., in press; Tinker, 2000).

Commonly, in the developing world, women are absorbed into their husbands' extended family and used as reproductive agents. Women typically have no power related to their reproductive rights and are accorded lower status in their society simply based on their gender (Burke \& Shields, 2005; Grown et al., 2005). “...family forms are buttressed by law, custom and ideology, and all of these are the fruits of political activity (O’Brien, 1981, p.92). 
Perhaps the most ominous commentary on the role of women in the family is the repeated violence perpetrated against them. Violence toward women in the developing world is very common in both physical and emotional forms (Ackerly, 2000; Craft, 1997; Fikree, et al., in press; Germain, 2004; Meleis, 2001; Pallitto \& O’Campo, 2004), and may be perpetrated by a husband, mother-in-law, or other family member. The reasons: perceived infractions related to roles and functions. This can be related to an insufficient dowry, inability to conceive and/or carry a pregnancy to term, or a perception that a daily task was not carried out in the proper way. At one hospital (over a period of seven weeks) students witnessed six young women for whom life had become intolerable; the six had ingested a weed killer (very accessible and cheap). Each died a slow and painful death. For these women this was their only escape even though it meant leaving their children behind. Students learned that although many families were open and trusting and had tendered family membership to them, there was still a greater societal problem generically relating to women and their status. Students worked with women who were labouring for the 6 th or $10^{\text {th }}$ time, and only had two or three living children; their perceptions about status in society and women's roles were piqued.

\section{Government Structures and Influence on Health Status}

Students learned government structures greatly influence the health status of families and communities. There was little infrastructure that filtered out to rural communities. Government tended to be centralised and decisions made at the top levels, with little “wiggle” room for local officials (Fuse \& Crenshaw, 2006; Schultz, 2002). At three of the venues students met with the mayors and spent time in conference learning about health policy and financial provision. Local government officials were often frustrated because it was felt that their tax monies were being spent at the national level and very little money was channelled to local authorities. There was discussion about HIV-AIDs and fears of its exponential growth directly related to lack of funding to conduct public awareness campaigns and treat patients. 
Both the formal and informal systems of the institution of government greatly affected the lives of childbearing women of poverty. Those in positions of power implemented rules, regulations, and mandates that influenced both individuals and groups. These mandates were interpreted and enforced in different ways in different locales. People, especially women, with no or little education, of low socioeconomic status, and without power within their own families were vulnerable because they did not know how to negotiate the rules and regulations. Students observed how this powerlessness was continually reinforced through the sheer numbers of pregnancies that each had during their childbearing years.

Health care funding was a crucial issue, due to economic market failures, World Bank debt repayment, and central government authority rules and regulations. Institutions, at all levels, had minimal local governance with decision-making capacities, and few resources (Filmer, Hammer, \& Pritchett, 2000; Sachs \& McArthur, 2005). Students learned how to work successfully, through being creative and adapting themselves to resource poor communities. Two "neonates" were made of discarded cardboard, one "healthy" and the other "dehydrated" or small for gestational age. Together the "babies” were used as props for hospital and community teaching/learning. "Rafelilto" was named by a patient, and made of a pillowcase stuffed with rags. He was a very successful "breast feeder” who accompanied us to 30 community sites. Amazingly, at each remote community, prior knowledge of Rafelito's existence was known, and was a wonderful trust building tool.

Government investment in health care provided some basic care. Additional services, especially reproductive health services, reinforced gender health care inequalities (Mehrota \& Jarrett, 2002). For example, on the issue of abortion, many of the childbearing women who annually die, their deaths are directly attributed to illegal and illicit "back street” abortions (Awareness mobilization, 2000; Rogo, 2004). This is due to institutional health care failing to provide such services (Burke \& Shields, 2005; Mhlanga, 2003; Pillai \& Gupta, 2006). Abortion is outlawed in many developing countries, yet desperate women seek this form of pregnancy termination. Family planning is another example of health policy that is very 
restricted and restrictive. Women were excluded as "informed" patients as men make the decisions on family size, and child spacing (Mhlanga, 2003; Sloss, Mirsky, \& Radlett, 1998, Szwarcwald Tavares de Andrade \& Bastos, 2002). Students worked with many women who were distressed with once again being pregnant and felt completely disheartened yet did not have access to family planning. Governments omit services, or limit funding, which allows the status quo to continue (Okojie, 1994; Sachs \& McArthur, 2005; Vlassoff; 1994). Ahmed et al. (2000) stated:

Gender is the socioculturally constructed role of the men and women in the society in contrast to sex, which refers to biological differences, and places a variety of expectations and constraints on women. It influences the health of women by putting emphasis on women's reproductive roles, resulting in early and excessive childbearing; sex preferences manifested in discrimination against female children in health, nutrition and general care; lack of autonomy leading to lack of decision-making power and independent income and to violence and abuse, causing severe repercussions on health and self-respect. (p. 362)

The governments of the developing world have a powerful influence on poor childbearing women (Cheru, 1992; Galeo, Freudenbert \& Vlahov, 2004; Germain, 2004) and students realised the potential of power, and the effects that any government can wield in any country. This next vignette depicts the labour and delivery "suite" of a hospital that averages greater than 50 deliveries every 24 hours.

\section{/Suture Material}

Thirteen women in one large room all in varying states of the second and third stages of labour; plus four more women in a smaller second room, opening into the main labour and delivery area. Nine midwives, one obstetrician, two registrars, six midwifery students, two instructors, plus administrative services, with three in staff, a typical day or night in the delivery suite of a public women's hospital serving poor local childbearing women.

Funds for hospital operations are in short supply due to the economic state of the country. Remnants of suture material used for laceration and episiotomy repair are kept in a 1500 millimeter jar. There are five centimetres of antiseptic solution that remain in the container, and suture material lies haphazardly, from the bottom to the top. Midwives and doctors routinely place their hands, or "sterile" 
instruments inside the container to obtain what suture material they need. I reflect: Is it better to (re)use when fresh, with all the colonies still thriving and multiplying, or is it better to dig deeper and use older remnants. The room temperature and the humidity match at approximately thirty seven degrees Celsius; the sun never reaches any of the supply containers.

For females in this country with its strict code of sexual conduct for women, and the double standard for men, and the recent arrival of HIV/AIDS, plus hepatitis, and myriad other infecting organisms, it does not bode well.

I often reminisce and deliberate the merits of the middle European legend of the stork delivering new babies to families, the mysticism surrounding this fable and the many generations of children who were taught this "magical" approach to birth and family expansion. The particular stork in question, huge, beautiful, as real as life but only a taxidermy specimen, is displayed in a prominent place in the entry foyer to the maternity hospital in (name of city). This is a treasured possession of this institution, replete with the stork carrying the new baby in a cloth dangling from its beak. I remember standing directly in front of the glass display case in a sort of dream state, full of magical thinking, philosophising about my intrapartal mothers in the developing world and the definitive benefits that would accrue from this use of "vaginal bypass”; how many maternal lives would be spared, how many neonates protected from the trauma of prolonged labours, maternal/foetal cephalopelvic disproportion, exhausted mothers, anaemic and tired, their bodies worn out by the constant repetitious cycle of pregnancy and birth. The next section will speak to religion and its place in women's lives.

\section{The Centrality of Religion}

Participants were not surprised to find religious practices were central to the lives of many communities and women's roles. At one site women partook in a religious ceremony that required fasting for a 24 hour period; a practice that took place on a weekly basis. Students talked to women to find out all about this religious tradition and then shared some of their own religious practices. At one venue students were amazed to learn of the ritual sacrifice of a goat in honour of a holy 
day. Intellectually students knew about this religious practice but the emotions can be overwhelmed when actually watching the animal being led into the building in preparation for the ceremony. The meat was then divided among community members on a rotating basis so that all could gain sustenance.

Religion affects the daily functions and overall role of women, and their young children across the life span (Ahmed et al., 2000, Carroll, 1983). In many instances religion and culture are intertwined. It is difficult to sort out what has lead to perpetrating certain cultural traditions that are interpreted, in individual countries, as purely religious prescriptions. One example is female infibulation, which under the guise of religion, results in sexual disfigurement and mutilation in order to control women's so called carnal desires and activities. Another "religiously” based cultural adaptation includes giving women's mothers-in-law absolute authority in deciding infant feeding practices, household duties, and food selection. Students saw young women continually deferring to their mothers-in-law and seeking permission to alter a day's routine. They watched the respect that was always present and were awed by the diffidence of the young women.

Yet another example was the standing in which men are held. Men, on the basis of religious dogma, and culture are the sole decision makers in families. In some cultures women cannot even leave their homes without permission to do so (Mumtaz \& Salway, 2005). The violence directed at women is often quoted as coming from God, as men are the true leaders and women should be the followers. It is the right and obligation of men to control their women and keep them in their place according to religious tenets. Students became aware of the openness surrounding violence perpetrated against women and the powerless of those being abused.

In South America "where the Catholic church is growing fastest, current dogma places an intolerable burden on women of reproductive age and it is the poor who suffer most” (Drife \& Kunzel, 2005, p. 1). Students learned about women's roles in relationship to reproduction and found the religious duty of women was to produce many children throughout their childbearing years. Students practiced at hospitals and in communities with grand-multiparous patients. Although they knew 
about large families from the mass media, actually knowing the individuals involved awakened their senses to differing ways of life. For students this all came together in “seeking health care”.

\section{Seeking Health Care}

The major reason traditional sociological institutions of society are addressed is because these five institutions most influenced the perceptions of students. The five institutions come together at the point where poor childbearing women access health care. When these women seek health care, they go to clinics, health stations, hospitals, or their local traditional birth attendant (TBA). They seek assistance from midwives, nurses, or doctors. Economically weak, uneducated, guided by culture and what has always been, they often go long distances to get health care.

In hospitals in the developing world, a physiologic health care model takes precedence over more holistic health care. The psychological, social, and cultural components of a woman's life are largely ignored. Women are ordered about, told what to do, and are expected to immediately comply with the directive(s) of that facility. They are sometimes punished for what the care provider perceives as inappropriate behaviour(s) by being struck, or yelled at. It is the norm to separate labouring women from their families throughout the labour, birthing and postnatal processes. For clients in a typical hospital postnatal unit, visitation hours are strictly regimented and rigidly enforced. Students became quickly aware of the enormous difference in focus; in their practices at their home institutions the psychosocial components of care were integral to the care that they offered.

In both the developing and developed worlds the experience of health care is largely influenced by the relationship women have with the providers in the health care setting. In many developing world countries, health care provision for poor childbearing women is a mandate by governments and providers must offer their services. Participants recognised the dual practices of obstetricians, private pay patients in the doctors' purpose built facility versus practising in government hospital facilities. Procedures were implemented as they have been done for decades. There was little innovation, or research, and few uniform policies and 
procedures to guide practice. In the developing world, government hospitals are largely understaffed, financially stressed, and lack clinical experts (Rosenfield \& Schwartz, 2005; Tinker, 2000).

In these health care settings, women and children who are poor and/or members of a minority community consistently have higher rates of maternal, neonatal, and infant mortality and morbidity (Fuse \& Crenshaw, 2006; Kurland, 2000; Lawn, Cousens \& Zupan, 2005; Sachs\& McArthur, 2005). As impoverished and marginalized, they are victims of stereotypical disparities of care (Bodley, 1982; Burger, 1987; Davis \& Glass, 1999; Foliaki \& Pearce, 2003). Those who can afford medications and treatment receive them, if available. The remainder of childbearing women go without interventions, such as analgesia, antibiotics, intravenous solutions, suturing, positioning, or basic comfort and hygiene measures. Students learned about the reality of poverty for women who could not afford medications for themselves or their sick neonates. Other examples of developing world hospital care included admonishing clients for waiting so long to bring their loved one to hospital and being told they were "stupid" because they could not understand what was happening. The childbearing poor are talked to, and about, in derogatory terms, while they are present, to other personnel. Health care providers, when working with these patients, carried on social conversations as if the clients were not present. Patients were berated concerning their ethnicity, their gender, the way they lived, and are told, "you should know better” than to wait so long to come to hospital. Patients were criticised because they become pregnant again and again. It was a vicious cycle, totally blamed on the childbearing patients. Participants were challenged yet again as they saw differences in care accorded to patients depending upon their "status". Each was deeply affected by the denigration of clients.

Health care providers in developing world institutions are trained to focus primarily on physiological processes related, in this instance, to childbirth. Doctors, nurses, and midwives could be abrasive. Many of the health professionals were placed in venues where they would rather have not been practicing. Because of this non-voluntary placement, disgruntled health care workers commonly displaced their feelings onto the recipients of health care, providing highly critical yet substandard 
care. Many young doctors, nurses, and midwives upon graduating must do a year or two of social service for their governments. There were often great disparities between where the health professional would have liked to practice and where they were actually sent for their period of government social service. Still others were employed by government health care services and were periodically moved to other service regions. By relocating health care providers, the venue, the clientele, and the practitioner were often at odds with one another. Students were well aware that they themselves were treated with dignity yet constantly saw the opposite for their patients. In this section I have addressed students’ perceptions and poor childbearing women in relationship economics, education, family, government and religion. I will now address the research question,

How did the international immersion programmes impact the personal and professional lives of student participants?

Study participants described the profound impact, through alterations in their perceptions, on both their personal and professional lives of participating in an immersion program. In the personal realm, students experienced a wide variety of conditions. They learned, practised, and adapted to local living conditions. They ate an array of different foods which provided little dietary variation; students slept on mats or basic cots, rather than beds; they shared small sleeping quarters with as many as eight other students. Students used toilets that required a small pail of water poured into the bowl in order to flush. They also acclimatised to outhouses with seats at angles that precluded attaining any level of comfort, trying to balance themselves over lopsided stone holes. Living areas had many insects, and at many venues mosquito netting was mandatory.

Participants attended religious services in Orthodox churches, Mosques and Hindu temples. The several hours spent standing, a tradition in the Eastern Rite churches, was a revelation for many. The women of the church loaned students headscarves for the services and made them welcome each time they attended. Students were continually a part of the social fabric participating in the cultural and religious traditions as locally practised. 
On a daily basis students participated in scenarios, and were confronted with dilemmas that challenged their values and expectations. Francis (2004) emphasised "it is in the taken-for-granted that embodied beliefs, values, personal biographies, culture and gender as 'normal' are most likely” (p. 244) to be explored, questioned, dissected, and reflected upon. Daily, professional and social constructs became part of the myriad topics that were routinely discussed.

Students had very few personal possessions, essentially no personal space, and often did not speak the local language. Informants encountered free roaming animals, and other infectious agents; they lived without typical American (USA) creature comforts such as furnishings, indoor plumbing, clean water, steady electricity, showers and baths, refrigeration, etc. Daily schedules were organized by the students to include household chores as well as work schedules. Sweeping the earthen floors with a twig broom, burning used toilet paper, washing and hanging out clothes to dry over barbed wire rails, dusting and washing living quarters with a local soap product and cooking on a kerosene stove, were some of the activities that needed to be organised in order to have each day flow smoothly. Activities of daily living took on a new "face" as an extraordinary amount of time was involved in order to meet the "life" demands of each day.

At one site, linen was changed for participants, though students were very uncomfortable with this arrangement. When they took over the function, they were flabbergasted to find multiple colonies of roaches on the underside of their mattresses. At this venue there was a saying that "only rich people have roaches" the implication being that the orphanage must then be a place of plenty.

The constraints of everyday life in the developing world highlighted profound environmental differences. Participants adapted to available diets and were astounded that having possessions were much less important than socially interacting with people, of establishing relationships. The scarcity of water and not being able to shower on a daily basis, or bathing and washing one's clothes in the river along with other community members, became important personal and social issues that were easily managed. Washing dishes in collected rainwater, or in the river, walking long distances to reach destinations amid forests, upon muddy or 
dusty narrow paths and fording rivers, sans bridges, were everyday "normal” occurrences. Each student learned the importance of the conservation of water as well as the importance of its consumption. Dehydration was of serious concern given both high temperatures and a lack of potable water. Students were always amazed at the scantiness and extreme concentration of their urine, due to their chronic state of diaphoresis/fluid deficit situation but they coped, and coped well. Thus daily life continued, with many notable differences from participants' usual lives as "privileged" first world citizens.

There was something profoundly reinforcing to students who were able to live and work in 38C degree temperatures with 98\% humidity, and still keep their focus. Students tolerated climate extremes, poverty, dirt, weather, animal encroachment, noise, silence, crowding, and darkness. They got up before sunrise and went to bed long after sunset and they loved it.

Despite the poverty, participants described the physical impact of going abroad in nearly universal positive terms. They felt strong and healthy. They described being able to live with fewer possessions than they thought possible. They were excited to be able to work from dawn to dusk, or into the night, without complaint, needed less sleep than at home, and could adapt to nearly any physical demand that arose. And, surprisingly the impact persisted long after the trips were over.

Students learned all about lifestyles that were vastly different than what they were familiar with. Families they encountered were cohesive multigenerational units where the roles and functions of women in relationship to other family members and the world outside the family were especially critical. Students were surprised at the importance of having male children rather than female and at times, the open violence perpetrated against female family members.

On the "home front" students were accepted as family members and included in family functions. As participants explored their village, town, city, they saw children and adults who had careers as beggars. They saw dogs that were homeless, and hairless, due to multiple infections, and skeleton thin. At a few of the venues they saw physical abuse of all species of animals. Most of all the students felt the 
warmth and acceptance by local populations as they engaged in everyday lives and routines within "their families". Their hearts were opened and they became more appreciative of the simple things in life, a protective roof over their heads, potable water, simple clothing, and three meals a day. Their outlook was changed as they were bearing witness to the reality of the life of millions of people who were poor, powerless, and citizens of the developing world.

These same informants were greatly impacted in their professional lives as well. In the immersion countries, there was little to nothing in the way of technological or routine equipment in the hospitals and community health centres. After using gloves, the gloves were routinely washed, hung out to dry and then autoclaved after use until they fell to pieces. Tubs of "sterile instruments" had multiple users who utilised a set of tongs kept in a separate "sterile" solution. Few patients had bed linen and held up to two patients including, at times, their babies. Syringes and needles were repeatedly reused, and at many venues, patients had to bring their own. Patients had to purchase their medications at pharmacies, and for those that could not afford to do so, they did without. Nursing functions included making individual dressings out of cotton wool and a soft covering, which were then autoclaved prior to use. Students practised nursing, as did the nurses at a particular venue, utilising what local resources were available. Many intense conversations ensued between the participants and the nurses at each of the sites. The topics discussed included comparing and contrasting the role and functions of nurses in the host community versus the participants' home community; families and life in general were also compared and contrasted in a very caring manner. Participants learned to do without equipment and technology while engaged in professional practice. They followed nurses who engendered health by touch, by voice, by caring, and the students did the same. The nurses positioned themselves in a willingness to be at the bedside of the most critically ill or "healthy" patients forever giving of themselves. Students again adopted the value of a genuine presence with patients and families above all else.

The participants who are now nurses continue to routinely question the disposability and rampant waste that they confront everyday in the health care 
system and find it very frustrating that their colleagues “just don’t understand” that it could be done differently. Students also talked about making what was needed, finding supplies to aid patients, and bringing in food. The camaraderie of the nurses at the immersion sites was inspiring unlike the USA where participants, currently practising nurses, feel there is no time to do the caring work of nurses in their present professional jobs due to simultaneously working with multiple patients of high acuity who are dependent upon "hi-tech" nursing care.

In their health care role, students learned many things. They participated in innovative ways of problem solving by practising nursing with their counterparts in the host country(ies). They worked side by side at hospital clinics, labour and delivery areas, surgical theatres, hospital units, and health centres. In time, the students provided care and resourcefully adapted to their clients' and their families' needs. Students initially had romanticised versions of how clinical settings would present and the reality was not as they had fantasised. They realised that some rudimentary health needs were met, more so for urban dwellers than those of rural areas. Labour and delivery had fair outcomes but seldom was there treatment for the related anomalies such as helminthiasis, or anaemia of the patient. There were no social services to help patients who were experiencing physical or emotional spousal abuse, nor financial aid to buy food for the patients' families. Participants supported patients throughout labour and delivery by physical reinforcement, massage, and by their constant presence as no family members were permitted entry. Because families were never permitted entry into labour, delivery, or the postnatal units, students were the only initial witnesses when neonates were born with congenital deformities, or were low birth weight and/or preterm. Inevitably, the husbands blamed their wives, and commonly these newborns were left behind to die. Students expressed emotional issues in the form of frustration, sadness and tears at the death of a neonate or young paediatric patient from starvation or other malady. Participants also shared in the joy of the survival of "their babies" or their paediatric patients, broken bones healed, or miraculously recovered from serious illnesses.

The students participated in mass dressing changes in large treatment rooms where many patients were attended to at the same time. The students found it 
emotionally difficult to cope with the physical pain of the patients during painful dressing changes, débridement, or the reducing of fractures without benefit of analgesia. This was especially devastating on a large paediatric burn unit where water baths and débridement were carried out on a daily basis. Students worked tenaciously to maintain an aseptic environment, but it was more difficult to emotionally "endure the pain" of their patients.

Initially frightened by lack of language skills, students found that they could provide effective nursing care using non verbal communication. At the bedside, participants gave full patient care. When students didn't know the language, they engaged in hand and facial gestures, and paralingual sounds; these tools of communication were very effective as student and patient worked together. Students looked upon those entrusted to their care as partners, on a joint journey in trust and care provision. They found their professional practice was not language bound and nursing care could be provided without language.

Social awareness increased as participants were awe-struck at the importance of immediate and extended family and the family as a unit, versus the singular, I, family member in the developed world. The students experienced families performing toileting, bathing, and preparing meals for their loved ones in hospital (all units except maternity) as participants came to realise that hospitalisation was a family "affair". The ongoing continuing effects of family participation opened yet another arena of student learning as such embedded participation in patient care is not the norm in the Western health care system. Participants now describe how they encourage family involvement in patient care, and how it becomes an early intervention. In so doing, the unity of the family is maintained, lines of communication and care are opened and maintained, and hospital experiences in the Western world are rendered more family friendly and less scary.

Students directly witnessed the combined effects of racism, education attainment, religion, economics, government, and family on individuals’ health status and access to health care. Students wondered when the time would come when all people would be treated with dignity and justice, regardless of who they were (are). Students expressed their feelings, and shared their perceived 
“impotence” concerning people who were not members of the prevailing majority population and they witnessed, as common occurrences, individuals and groups adversely disadvantaged throughout their lives.

At the outset students anticipated and equated poverty with sadness and grief. Instead they found great joyfulness for the vast majority of people. Trust, the professional and social friendships that ensued, the camaraderie, the becoming part of families and their activities, were important facets of the immersion experience that had a continuing impact on participants. For example, students who were three to twelve years post immersion participation, still found that they were drawn to simplicity and economic discretion in both their professional and personal lives. They described themselves as person-focused rather than possession-focused. Whilst they have, for the most part, set up traditional homes, they still have values about the importance of time with family and friends, purposeful communication, health and health teaching, safety and security, and offering themselves to help others as opposed to their materialistic consumer oriented counterparts. One student described materialistic things as unimportant; they simply hold little value in her life both at home and at work. For this student who lived in an environment, during her immersion experience, that would be considered “abject poverty” by developed nations, the effects have been both powerful and intense. Seeing people happy in conducting their daily lives and children content playing with toys made of twigs and roots influenced how she lives today. This student felt that now, as a parent, her experiences in the developing world had multiple effects on how she and her husband are raising their children. They have daily family time together, parents and children, talking, taking walks, and playing games as a family unit. They value their family time together sharing their experiences of the day, cooking dinner as a family unit, reading together, emotionally supporting one another, and enjoying themselves as an integrated family. They mirrored the model they had experienced in the developing world, not one commonly seen in the USA today. I furthered reflected on the following research question,

Can the student experiences help me to understand myself better? 
I can emphatically say YES. As I became conscious of the impact of the programme on participants, I began to understand myself more clearly and my commitment to my students and their need for exposure to other. The immersion programme journey was a powerful one for each individual but even more powerful for me. The journeys continue to tug at my heart, have always challenged me professionally, moved me emotionally, and have continued to reinforce the great divide between the have and the have-nots.

The most profound personal impact was a fundamental transformative life change. Before the students went to foreign countries, most saw themselves as progressive and liberal, open and accepting. What happened in the course of the immersion programme was that they realized they were not truly any of these things. They were not progressive - they were well entrenched in the values and beliefs of the USA and they were not as liberal as they thought they were. They were "open" to the American way, but were astounded that the rest of the world didn't function that way. This was a further blatant cue for me of the necessity of exposure and experiential learning. Students were rooted in the majority culture, lacking exposure to genuine differentness. They also were participating members of a powerful, wealthy and privileged first world country. This means to me that there was an incredible need for exposure to "the other side of life".

During the immersion experiences, students were exposed to patients that were completely powerless and disregarded due to their refugee or minority status, related to poverty, race, religion, or ethnicity. This brought about the "illumination" that powerful discrimination exists both overseas and in the USA. Students were troubled as they considered that they were part of the system, perpetrators, of this kind of health care provision without realising it. For me this further solidified the need to continue immersion programmes, and to lobby for all students to be exposed and immersed beyond their known psycho/socio/cultural boundaries.

Seeing class structures and differential care caused the students to be aware of who they are and how they represent themselves. They became, and have remained, willing advocates for all clients. They described the need to speak up when critical comments are made about non-traditional or cross cultural patients, 
both then and now. One student participant was recently horrified when an elderly Native American client was being forced to remain in hospital to die rather than being at home surrounded by extended family. This student spoke up for this patient, advocated and fought for the client's rights, and secured her privilege to pursue her cultural tradition by dying at home. It took a great deal of energy and commitment to do the "right thing" for the patient and her family. Advocacy must be an essential element of the nurse's role and one that I have always engaged in most actively, at times, getting me into "trouble". I am a staunch student advocate and teach to the role of student advocacy for their patients and their families.

Participants described how the relationships they established, then and now, remain the most important work that they do - over and above the technical or basic physiologic patient care routinely completed. Participants talked about trust, partnerships, mutual health goals, empowered and knowledgeable families, caring, supporting, nurturing, advocating, and each client being a highly valued individual with her/his unique background, beliefs, perceptions, and needs.This is who I am. It's about people and relationships, not anything else.

Then and now, the life changing impact of stepping outside one's comfort zone into the world of others changed the hearts, work ethic, values and lives of participants in nearly immeasurable ways. This helped me understand the importance of creativity, flexibility, and innovativeness within the professional and personal lives of students and the need for promoting this. The student experience helped me realise the importance of international immersion programmes in human developmental milestones. This helped me understand myself to an even greater depth in the realisation that I must continue this outreach for my students. I also understood that I needed to know more about the many concepts related to culture and just how deeply each is affected by culture and in order to know oneself one must know "culture”.

\section{Culture: To Know Oneself}

I have been challenged in my thesis reading, writing, and research concerning the meaning of culture. In my education in the USA I was exposed to 
Leininger $(1978,1979)$ and her concepts of Transcultural Nursing. It was proposed that one needed to learn everything about another culture in order to be able to nurse culturally appropriately. Years ago I came upon the writings of Ramsden (1990, 1995, 1996) and initially was stymied when reading about the political processes related to access to health care, the psychosocial process involved, the role and functions of stakeholders, and the revelation of clients' issues related to revealing who they truly are. What I have discovered and now understand is my life's work is getting students to understand themselves and their own culture in order to be able to nurse everyone appropriately.

To define culture absolutely is an elusive prospect that is both challenging and complex. Suominen, Kovasin and Ketola (1997) asserted the essence of culture "has to do with the deep and invisible structures in society which are transferred from one generation to another" (p. 186). Kroeber (1952) envisaged that "values constitute an essential ingredient of culture [and] they are subjectively held” (p. 5). Kroeber \& Kluckhohn, (1952) put forward the concept that the term culture was complex and multifaceted despite there being no general consensus of its meaning. Linton (1955) stated that "No one person is ever familiar with the entire culture of his society” (p. 33) and I agree.

Culture continues to be an evolving concept. Kroeber and Kluckhohn (1952) found more than 100 definitions of the word culture. Culture, and all its myriad nuances, meanings and ramifications still do not have one definitive definition. In the context of today's usage, culture includes the ways of life that are handed down, and practiced, through generations of a family/community/group/society/ nation. The word culture first appeared in British and American dictionaries in the early $20^{\text {th }}$ century and in the German lexicon a century earlier. Up to that time, it was usually defined in relationship to soil cultivation, the arts, or describing the aristocracy (Kroeber \& Kluckhohn, 1952).

Culture is a curious phenomenon. We immerse ourselves transculturally everyday, without thinking about it, often without the realisation of what we are doing. We cross cultural boundaries, such as belonging to an organisation, club, or other group, all with their own "culture”. Rosaldo (1989) termed this "border 
crossings”, defined as “...crossing social boundaries in our daily lives” (p. 29). Yet when we cross other cultural boundaries such as race, language, and ethnicity, the issue can present a conundrum. The conundrum can be a personal perception of vulnerability and powerlessness due to the anxiety and fear of the unknown and the perception of challenge to the status quo. Though we participate in many multiple cultural organisational structures that are accepted without much question, "social systems" that are deemed "foreign to us" result in our feeling threatened. Stated differently, there are values ascribed to a particular organisation that we partake in, yet when these values pertain to individuals or groups that are “different”, problems arise. As we live each day, we participate in multiple experiences and relationships, each interaction familiar to us in our everyday mundane situations. Rosaldo contended the "classic norms of anthropology have attended more to the unity of cultural wholes than to their myriad crossroads and borderlands” (p. 30). Rosaldo's concept is very important because each of us is an individual. Though we may share a particular society’s “cultural values”, it is necessary to separate out the individualism of each person when providing nursing care and caring.

Kalantzis (2000) described how "the local becomes global as immigration transforms once-homogenous communities into global communities” (p. 100). The idea of culture expands and emphasises diversity at a grass roots level. For local small communities this engenders individuals and families interacting with one another on a personal level in multiple roles. The significance of this physical closeness is that traditions, languages, foods, value systems that are new are introduced to individuals. These same individuals who in the past would not have come into contact with such an enormous amount of diversity absorb "new ways” of interacting with each other. Kalantzis described each of us as "members of multiple and overlapping communities-communities of work, of interest and affiliation, of ethnicity of sexual identity, and so on” (p.107). This is important because, again, we must recognise the individual and his/her belief systems rather than making assumptions about the person. She further stated that "each of us has an identity composed of multiple layers, simultaneously a member of multiple lifeworlds” (p. 107). 
Goodenough (1981) characterised culture as a "system of standards for perceiving, believing, evaluating and acting” (p. 110). Barer-Stein’s (1994) definition of culture is the most encompassing: "All aspects of the patterns of daily life that are learned by an individual and that determinedly affects that person's behaviour. [It] provides a sense of order, security and identity, and yet paradoxically is in a state of continuous change” (p. 150). As we live, we are constantly exposed to newness in multiple forms and as a consequence we are constantly evolving, changing, and/or altering some miniscule part of ourselves which becomes entrenched in one's “personal culture”.

The dilemma of culture and cultural identification is embedded in "the evolution of the individual". We are not static beings, and as we "ebb and flow" in our daily lives, we change. We adapt, adopt, alter, and revise ourselves through cultural experiences and persistent exposures of everyday living, and for nurses this knowledge must be fully explored in their daily working lives. As nurses our actions with patients can be directly and negatively affected if we make assumptions about people because of what is perceived as "their" culture.

The basis of the profession of nursing is the relationship between patient and the nurse. This relationship "is considered the foundation of nursing care, the context in which nurses practice” (Hagerty \& Patusky, 2003, p. 145). In order to understand patients, nurses must first, and foremost, understand themselves (Leenerts, 2003). This is pivotal to a nurse-patient therapeutic relationship because "personal knowing, with emphasis on subjective experience and empathy remains critical to healing” (Leenerts, p. 160). In providing culturally appropriate care and caring for patients and their families, nurses must know who they are and address their interactions, in such a way as to accomplish a genuine caring bond.

Few things are more difficult than to see outside the bounds of your own perspective-to be able to identify assumptions that you take as universal truths but which, instead have been crafted by your own unique identity and experiences in the world. We live much of our lives in our own heads, in a reconfirming dialogue with ourselves. (Takacs, 2003, p. 27)

By having deep, profound personal knowledge about ourselves and truly knowing “who we are”, we can be more flexible, adaptable and open to others without feeling 
threatened by differences. The priority of nurses seeking to be culturally competent ought to be exposure...TO ONESELF, because knowing, I, enables the nurse to "provide the catalyst for the passion, authenticity and wholeness that is essential to therapeutic relationships” (Leenerts, 2003, p. 160).

The instrument that I have chosen to use with students is an international immersion programme. The ostensible objective is learning about other cultures, and lifeways, however, students truly learn about themselves, their modus operandi, and beliefs and their value systems. Students' senses are engaged in perceptions new to them that provoke a process of questioning, reflecting, and re-visioning of life, culture, and the profession of nursing. Nurses must first know their very "own” personal culture and how it colours who they are and how they interact with patients and families. Sometimes one’s own cultural values are only understood after stepping outside one's own boundaries. For many students exposure to other has been limited and assumptions are made that "all the world" is like us.

Immersion programmes move beyond (should be beyond) cultural awareness and competence by students being immersed into a new "context" and "confronted with...rapid change in circumstances” (Mezirow 2000, p. 3), known as experiential learning. The programmes have the potential to be emancipatory in the sense that one can learn about oneself, understand oneself, accept oneself and others, and recognize social dialectics. According to Perry’s (1970) theory of development “any situation or experience that is incongruent with an individual's current reasoning structure may serve to challenge established views and stimulate...change” (as cited in Zorn et al. 1995, p. 70). This includes immersion, challenge, and risk taking, as well as transforming cultural perceptions.

There are "simple" cultural awareness(es) that are observable symbols of a culture, among them, distinguishing physiologic characteristics, code of dress, food, and eating practices, music and music making (Purnell \& Paulanka, 2003). A nurse researcher, Camphina-Bacote (1999) defined this awareness as "the deliberate, cognitive process in which health care providers, become appreciative and sensitive to the values, beliefs, lifeways, practices, and problem solving strategies of clients' 
cultures” (p. 204). Camphina-Bacote (2003) further stated that nurses must always initially and deliberately examine their own way of being.

I concur with the premise that "knowing one self first" must always be priority number one. The most important entity that the nurse can offer others is her/him-self. It is I, rather than "thou” that has the greatest impact on care and caring, meaning that the nurse's practice is directly affected by who she is rather than who the patient is. It is what the nurse "brings" of her/him-self to the clientpatient relationship that is pivotal (Benner, 1999, 2000). If you know who you are, you will understand your own reactions, thoughts, and feelings. This in turn affects interventions in professional relationships. Peplau (1952) stated "being able to understand one's [the nurse] own behavior” (xiii) is crucial to a positive patientnurse relationship.

Ramsden’s (1995, 2000, 2002) Cultural Safety Model asserts the importance of the social, political, economic and historical issues of people and their effects on health across the life span. Carryer (1997) asserts “people cannot be effectively nursed without reference to their environment. This may be their family, their wider social sphere or more ambitiously the socio-political context in which each individual negotiates their health” (p. 168). Colonisation has affected millions of the world's people and the effects are still reverberating around the world. Colonialism is defined (Flexner, 1983) as "The control of government influence of a nation over a dependent country, territory, or people... The system or policy by which a nation maintains or advocates such control or influence (p. 405). Colonialisation is also defined by Flexner (1983) as "the act of bringing into subjection or subjugation by colonizing” (p. 405). Colonised people suffer disproportionately higher disease rates, have a shorter life span, have less economic opportunities, have less access to higher education, and are not proportionally represented in the political arena of their countries (Chapman \& Berggren, 2005; de Costa, 2001; Durie, 1994, 2001; Ramsden, 2002; Subramanian, Jarvis, Chen, Rehkopf, Waterman \& Krieger, 2005; Tamasese, 1998; Waldegrave, 1999, 2000). In this way, they are made second class citizens. A classic example is the Maori people of New Zealand who have suffered greatly from colonialism. They are marginalised and have disproportionately higher 
chronic diseases rates, die younger than pakeha (a Maori word used to describe Anglo-Europeans of New Zealand), have a lower socioeconomic position in society, and complete less years of formal schooling than other groups (Crampton, Salmond, Kirkpatrick, Scarborough, \& Skelly, 2000; Davis \& Dew; 1999; Durie 1994, 2001; National Health Committee, 1998). In the United States, the indigenous population, Blacks, and Spanish speakers from many countries, have also experienced similar socio-political, socio-economic, and health challenges (Cornelius, 2004; Gennaro; 2005; Howard, Ford, \& McLean, 2004, Ketefian \& Porter, 2000; Lopez-Alonzo, 2001). Disadvantaged minority groups have higher rates of hypertension, diabetes, heart disease, and poorer obstetric outcomes. Negative consequences, including socioeconomic, such as low waged and menial employment, and less formal education; psychosocial issues, include higher rates of mental health problems, and feelings of being on the outside of societal norms, impact their lives (Braverman, Egerter, \& Cubbin, 2004; Gennaro, 2005; Maze, 2005; Villarruel, 2004; Williams, 2002).

It is imperative for nurses to recognise and understand the potential institutional power [one's professional culture] that each carries within her/him-self. The "institution" represents the majority culture health care system and for people who are powerless in their society, vulnerability is reinforced in the health care system. Health practices of the majority population can be imposed upon minority peoples and may not be congruent with their life ways. The attitude of professional health care providers can negatively affect health status, treatment offered, and ultimately, health maintenance and promotion. Individuals, too, who are identified with a culture that differs from the health care provider [i.e. gender bias, homosexuality] can also experience difficulties (Schim, Doorenbos, \& Borse, 2005).

Nurses' attitudes can also negatively impact care, caring, and patients. Unless patients feel "safe" they will withhold disclosing their uniqueness or their needs to care providers. The effects of marginality and silence are detrimental to health because the patient does not receive the appropriate care (Ramsden, 2000). Nurses must meet the challenge by not assuming or assigning attributes to a patient 
because he/she belongs to a specific cultural group. Individualised care for each patient and family is necessary in order to optimise health.

Florence Nightingale (1859/1980) addressed the multiple issues of psycho/socio/economic, environment and culture which have enormous effects upon individuals and groups of people. “...the symptoms or the sufferings generally considered to be inevitable and incident to the disease are very often not symptoms of the disease at all, but of something quite different” (Nightingale, p. 1). The environment, diet, housing, lifestyle, employment, and education all affect who we are as individuals thus influencing our health care seeking and provision.

A century later, nurse-anthropologist Madeline Leininger, from the USA, emerged with her theory of Transcultural Nursing. Leininger (1978, 1979, 2002) and Leininger and McFarland (2002) discussed caring and how it takes different forms according to cultural beliefs. Her emphasis was the need to incorporate nontraditional practices, such as "folk ways", and cultural specific knowledge into nursing caring. Leininger (1978) and Leininger and McFarland (2002) also addressed the critical importance of nurses in understanding cultures in order to work effectively with clients and their families. The danger remains that assumptions can be made about an ethnic minority person simply because that person belongs to a particular group.

Alibahi (1986) stated, “there are so many cultures that no one can be expected to know them all and the result may be an oversimplified clarifying, and packaging of cultures" (as cited in McGee, 1992, p. 7). Bruni (1988) echoes the potential pitfalls of ascribing attributes to individuals by virtue of their cultural or racial identity: "If you assume an individual is like everyone else in a [specific] culture, you have stereotyped all the various people in that culture into one mold" (Jandt, 2001, p. 6). Another intervening variable is the idea that "diversity within cultures probably exceeds the differences between cultures” (Jandt, p. 6) which means that ascribing particular characteristics to a person because he/she belongs to a particular "group”, is stereotyping (Schim, 2005). An example would be caring for a Hindu client, and without consulting with your patient making assumptions about dietary patterns. Del Bueno and Vincent (1986) stated that culture is interpreted by 
the individual, thus “common ground” may be lacking when healthcare providers impose preconceived notions about the values of their patients without thoroughly assessing each individual's life ways and belief systems.

Nurses, in order to prevail in their role as health care providers, must look to themselves first (Leenerts, 2003). By really understanding self, all the nuances of I, the nurse is prepared to understand others including their culture and life style. The nurse also must know how individuals are affected by their history and place in society. The nurse must also have full realisation of her/his professional and institutional "power" and the overall effects of the sociologic institutions upon individuals and groups. As nurses we must meet our professional goal(s) by establishing the culture of each individual patient because if ignored, health care goals will not be attained. In the next section I will explore,

What is it that impels me to return again and again to the developing world?

Every year an estimated four million babies die in the first four weeks of life. A similar number are stillborn, and 0.5 million [conservative estimate] mothers die from pregnancy-related causes. Almost all (99\%) neonatal deaths arise in low-income and middleincome countries, yet most epidemiological and other research focuses on the 1\% [neonatal] of deaths in rich countries (Lawn, et al. 2005, p. 891)

Many personal feelings are engendered as I look around at American (USA) youth and adults who take so much for granted in their daily lives. They do not have a clue as to how most people in this world live. The majority of people, of any age, in the developed world truly do not understand the personal daily struggles for survival of the people of developing nations, among them, women of childbearing age and their young children. Yes, there are the poignant flashes across millions of television screens; yes, newspapers depict with words and pictures, the unspeakable conditions of daily life; yet the cycle of morbidity and mortality continues to be perpetuated.

My personal and professional journey has provided continuing indepth insight into the complex issues surrounding the processes that poor childbearing 
women undergo as patients in the health care systems of developing world countries. "To study lived experience means that the researcher studies the world as it is immediately experienced rather than as one theorizes about it” (DeLuca, 2004, p. 657).

The commonalities of child birthing experiences have been seen repeatedly. The experiences of women, in this milieu often include: lack of patient control, significant family disruption, disorganization of care, medical crises, anger of care providers, unequal health care, lack of resources, low levels of patient and provider education, harshly critical staff members, grief and loss. Despite these negative happenings, the most fundamental of human experiences are graphically played out during the birthing process.

Nursing students of the developed world came face to face, with global realities. They needed exposure and experience with lives that were different than theirs because “the classroom and 'real life' are inseparable” (Olbrys, 2004, p. 9). They needed to expand their world views; develop their own values; become political, experience being a minority; and provide "caring care” to those, who by virtue of their place of birth lead diametrically opposite lifestyles.

For those nursing students who have participated in immersion experiences a growth process took place revealing the foibles of humanity both in the host countries and the USA. It was/is painful, as one comprehends, and bears witness to the lack of basic humanity towards others, such as social injustice and indignity. One becomes conscious of the perpetration and perpetuation of realities that exist as part of the social fabric of any society. Students were stunned as they came to the realisation that these problems exist not only in the host countries but in their home country too. "No political, or counter-hegemonic invasions into schools can be made without eventually understanding one's relationship to social structures, which embraces one’s personal history” (Kanpol, 1998, p. 191). In the next section I reveal more of who I am. 


\section{Who Am I?}

My personal background has greatly influenced who I am, and my world view. Growing up in a multi-ethnic, racial and polyglot city, New York, I had the advantages of early and constant exposure to cultural practices of many diverse groups of people. The many New York City ethnic enclaves and neighbourhoods offered insights into a rich tapestry of life. My parents had the foresight to expose us to a wonderful array of diverse ethnic cultures, celebrations and lifestyles, encouraging us to participate in these unique activities on a routine basis. It was never feared that we would lose "our culture" via this exposure because it was their desire that their children should constantly and consistently be part of the richness of the life that surrounded them. At an early age my parents and extended family presented the world to me to explore, study, and cultivate because "when one is situated within one's own cultural experience there is a horizon or a range of vision that determines what can or cannot be seen” Gadamer (1960/trans. 1982, p. 19). This guiding principle of exploration and observation has been my modus operandi throughout my life.

My family viewed themselves, not as the centre of the universe, but as part of the "human family" with myriad ways of celebrating milestones, many ways of problem solving, and numerous approaches to family structure, roles and functions; each unique, each valid in the context of the milieu that supported it. This environment provided many contrasting ways to understand the meaning of unique ways of living life. When I became an adult, I extended my cultural geographic forays to include the world at large. This education, and my subsequent immersion into many different societies, contributes to my continuing understanding of people and the effects of culture on their daily lives. I also know that each person is an individual, regardless of how one is "assigned" to a cultural or ethnic or racial grouping and that the "variations" on lived life and beliefs are infinite. 


\section{Summary}

This chapter has focused on my thesis journey and the realisation that at the outset of the programmes I did not have any inkling as to the depth of change that would transpire for students. Students’ perceptions and transformation are presented. I have also addressed some thoughts on poor childbearing women and the societies in which they live. I have discussed the importance of culture in relationship to knowing oneself. Who we are, and how we professionally (and personally) communicate greatly affects our patients and their families and their ultimate trust of the health care system. I have also further revealed more of who I am. The next chapter will reveal my epiphany. 


\section{CHAPTER VIII}

\section{MY EPIPHANY}

"Mundus vult decipi....the taste for the truth is an acquired taste that few acquire"

(Buber, 1937/trans. 1970, p. 9).

\section{Introduction}

In this chapter my epiphany unfolds and the many major and minor nuances that have affected who I am and what I do are engaged. I also address more of my professional and personal background as well as reasons for providing international immersion experiences for students. Philosophical concepts related to my epiphany are spoken to as well as the more pragmatic loss of maternal and neonatal lives in the developing world. All these facets have led me to my epiphany.

\section{My Epiphany}

Passionate scholarship is profoundly related to personal beliefs (Minnich, 1990). "Passionate scholarship that is inspired neither by a desire to dominate, to control, to possess certainty, nor by external rewards emerges from people who are pursuing their own questions” (Minnich, 1990, p. 163). In Humanistic Nursing (1976) Paterson and Zderad stated: “one’s past would be visible in view of how one approached and experienced the present” (p. 105). Meleis (1987) posited that to be personally accountable each of us must search "for the meaning of our research for ourselves" (p. 16). Goodman (1998) emphasised "scholarship is most useful when it helps its readers to gain insight into the human condition in ways that are personally and socially meaningful" (p. 55). I am "the filter of salience through which data are sieved” (Stern, 2001, p. 60).

From my beginning awareness, "I have often wondered what meaning I can take from my youth [my life]” (Kamphol, 1998, p. 194). What are the factors that 
have sculpted and moulded me into the person that I am, doing what I do today? There are multiple factors and an abundance of people, over my lifetime who have been involved in the process.

I embarked on a five year journey of intense reflection, re-visioning, constructing and deconstructing my thesis. My realisation, my epiphany, took place when least expected (Moustakas, 1990). It is absolutely simplistic and yet I never realised what it was, and the sway that it holds over my life as it transmits, conveys and communicates to students and to me "the power of one" to endure. I care, I facilitate, the medium being the immersion programmes; thus I provide the vehicle, thus I am the intermediary to life changing transformation (metamorphosis). I do not cause change I provide the environment for the most basic change that people can experience, a transformation in their perception of humanity. That is the epiphany. Metaphorically speaking I provide the stage, the actors are living their lives, "playing their roles", as "the window to the world" is opened, traversed, explored and experienced. The last act, the finale, the conclusion, is transformation.

Diekelmann $(1990,1995,2001)$ struck a profoundly resonant chord, loud and clear, as I recognised and compared my teaching style to narrative pedagogy, defined as "reflective thinking about common everyday experiences" (Ironsides, 2004, p.11). From this collaborative endeavour (with students) new knowledge, change, growth, flexibility, adaptability, understanding, and different ways of learning, interacting, communicating, and being in the world emerged.

I really had no idea of the profound changes that would take place in the professional and personal lives of student participants in my immersion programmes; the give and take of a dynamic, and ever changing set of experiences. I realised that the combination of who I am and the work of Diekelmann $(1993,2001)$ truly represented an epiphany, that the sum is truly greater than its parts. I suddenly understood the change(s) that each student experienced. As I watched and participated in students' daily lived lives, both professionally and socially in the host country(ies), a slow metamorphosis would take place. The gleaming and the gleening of the participants' discovery of how the lives of others were lived and the effects of culture (cumulative effects of economics, education, family, government, 
and religion) upon each person were significant. Students recognised that they too had a culture that affected their perceptions and actions. Participants identified emotional and mental processes that differed from theirs. Euro-Americans are a diverse group yet "their values are consistent with dominant values of the culture. Therefore, members of this group may not be “...aware of the role that culture plays in their lives as [do] members of other cultural groups” (Engebretson \& Headley, 2000, p. 294). At first differences seemed strange but as the world of the participants expanded they grew. They partook in activities that opened them up to the dialectic of differences and opposites. The many fragments, pieces and segments of the immersion experience meshed together and participants grew exponentially psychosocially. It was my hope that individuals would develop, beyond what they thought possible-to re-vision life as it is lived.

I was raised in a home where both discourse and the dialectic were constants: at the dinner table, on walks, during casual conversation, during very heated discussions. I was encouraged to always question and always push beyond the expected. Why this? Why that? Why are, some people, treated one way and others treated differently? I was brought up to challenge what were perceived as social injustices; race, religion, ethnicity were concepts to be questioned, not merely accepted on their face value. An example, a seeming innocuous comment, made years ago by a friend, about husbands (Caucasian) not wanting their wives to be burdened by "housework” that “coloured women” could do. I retorted that Black husbands, perhaps, had these very same feelings. I received incredulous stares from the group of people with whom I was dining; I don't think that they had ever really thought about the other. It is as if "through our [my] actions we [I] give expression to the personal and social commitments that we [I] have made (Denhardt, 1981, p.7).

I have always felt the pain of others, who, by virtue of belonging to a particular group are "globbed" together, stereotyped, stamped, and perceived as possessing certain negative attributes. I bear witness to psychological and collective pain when any human being(s) is demeaned, globally as well as in my own “backyard”, my country, my town, and my university. Each individual is a unique entity and not just a "member" of a particular cultural, ethnic or racial group. I 
become enveloped by the pain of others as I constantly remember the past, and feel in the present, the inequities of life.

Many years ago my older daughter and I were swimming at a beach in Sri Lanka when we were approached by a distraught man (Tamil) whose pregnant wife needed an emergency Caesarean delivery. The couple had been travelling outside of Jaffna province when she went into premature labour and they did not have the resources to pay for the surgery. They were unable to get back to their home territory and hospital in a reasonable time frame. The man's fear was real, and appropriate, as the civil war in Sri Lanka had been going on for many years. They were in Singhalese territory and he was terrified because of the overt hostility displayed toward him by both adults and children. I vividly remember the young children at the beach openly ridiculing him as he was Tamil and they were Singhalese. There were many derogatory comments such as "I hope that your wife dies”; “you are a Tamil and don’t belong here”; "who cares about Tamils?” I could not ignore this family's need so I accompanied him to the hospital and arranged for payment. Weeks later I received the money from this family to cover the hospital expenses. This was a human being in dire need, caught in hostile territory and for me there was no choice.

I think of my younger daughter and another incident that profoundly affected the two of us. At school recess one day she was climbing on the "monkey bars"; she was eight years old. A voice from another child rang out, "get off the monkey bars, black face”. It took my East Indian daughter six months to be able to share this with me. Being racially ostracised is a profound experience. My daughter is now 20 and still hears friends make racist comments about others, yet they appear to be colourblind to her. The stereotyping of groups continues, yet individuals can be perceived as okay, because the individual is told he/she is not like the rest of "her people". That has been my daughter's experience.

For those raised with multiple creature comforts that have read about and "virtually experienced" the impotence of the everyday lives and struggles of their fellow human beings, it is essential to gain an understanding of life from another vantage point. It is easier to "turn off”, ignore, the lives of others and engage in 
one's “ordinary" life than it is to actually "live” the experience(s) of another person(s). Some believe it is never possible, to either comprehend another's perceptions, nor experience their world and that the best any one human being can do is to meet where lives intersect (Thayer-Bacon, 2000). The following poem eloquently illustrates this point:

To know about the Others and not be able to describe fully To have knowledge and artifacts yet struggle to portray findings adequately To enter the Others world but only partially

To want full knowledge of Others but catch only a glimpse

To share but attain only partial congruence

To sit in awe of Others yet know, one from the Others' World would hear more

Yet know that what is seen is through a haze

How can one enter the Others' World?

The very core, spirit-life of the Others

Hush be still, you cannot---you are of Another's World The Others' World is theirs You must be grateful the Others afforded you a glimpse Not many people are privileged to enter the Others' World The Others risked their spirit-life when you entered Guard the treasure received as precious jewels You were permitted to partake of the Others' spirit-life. (Reeb, 1994, p.230)

At this intersection participants retain their own identity but share their humanity. What this means is we all are in the human family.

So the questions must be asked: Why do I return again and again to work in others' “worlds” and explore different cultural standards and lifestyles? Why do I take students? The answer is straightforward and uncomplicated. There are many people who live in this world, barely existing, yet their existence is not truly acknowledged. They are individuals, families, communities, people, with feelings, roles, and functions, just like us. Others also have dreams, aspirations, and experience the gamut of emotions each wanting to survive and provide for their children.

At times, in my ethical stance, I feel as driven as an artist is impelled to paint. I feel that I must expose my students and myself to the myriad ways one can 
be in the world. As humans we all share this planet and it is imperative that we learn about the ways of living of other people. For the students it is the total absorption, the immersion into the community and becoming part of the lives of others that bring about affective change. It is learning how to be accepted and feel comfortable with others who also offer themselves, as they are in their daily lives that promotes mutual sharing of lived lives. Can it not be the sheer joy of humanness and the contributing, partaking and involvement in the everyday lived lives that bring us together? In the relationship, any relationship, people are changed. They are changed; I am changed; my students are changed. “...we bring to every situation our usual habits of mind, our priorities, our general outlook, our usual modes of interior processing...the power to change perspectives and to view experience in different ways” (Anderson, R., 1989, p. 421), challenges and changes world views and lives.

It is also practising the profession of nursing alongside health care providers in a professional world that greatly differs from our own, yet, holds similar goals. These goals are health promotion, maintenance and restoration. What do you say, what can you say, when students come to you with statements that they hear: "those people are not human like us"; those people are worse than animals”; those people are so dirty”. Students working with majority culture health care professionals in the host country(ies) saw both members of the dominant and non dominant culture(s). They saw staff members discuss the indigenous population for whom they provided health care services in one way and describe in another way the members of the majority culture as the "norm”. It changed students. It changed their lives.

Thus began an existential process of reflection: "Who am I and how do I fit into this world?” This heightened sensitivity to others initiated an interesting intrapersonal and interpersonal dialogue. What happens when students are socialised into the health care profession via lived experiences? What happens with the lack of "perfect" health care, the lack of fit of provider and recipient, the STRESS that interferes with health care promotion, maintenance and restoration? What happens when people are poorly treated, and treated poorly? What happens with marginalised groups, dominant groups, or individuals? "Nursing is an experience lived between human beings. Each nursing situation reciprocally evokes and affects the expression 
and manifestations” (Paterson \& Zderad, 1976, p. 3) of the actors and if one of the key persons is rendered powerless, health status will be negatively impacted. It is this discrepancy in psycho/socio/cultural care provision that directly affects the humanity of the patient. Individuals, families, communities, and even countries suffer socially and economically if trust is not built between health care recipients and providers.

People suffer unnecessarily for being a member of a particular "group" (Davis \& Glass, 1999). Poor childbearing women are one such cohort. I care about these women. The women live with extended family; they live as female heads of households; they live in nuclear families. They live in rural areas, villages and towns, in cities and large metropolitan areas, in deprivation and in need.

Girls are programmed from early childhood for their adult roles as subservient wives, childbearing women, and mothers without formal education. From a very young age, girls are initiated into caring for their younger siblings, domestic work, and labouring in the family fields or businesses. The work day lasts many hours, and includes such things as fetching water and wood for cooking, washing clothes in the river, preparing food, and general housekeeping duties, childcare, extended family care, and tending a small vegetable garden and/or tending to chickens or a cow or goat. I care about these girls.

In the developing world inadequate housing for the poor is the norm. It is commonplace to live with human and other wastes, overcrowding, and little or no circulation of air in one's home. No access to potable water is also typical. Housing consists of paper shacks, thatched huts, corrugated metal, stones pieced together, plastic and cardboard props, or teeming dilapidated tenements. Very few houses have furnishings and inhabitants sleep on the floor, a pile of hay, or other make-shift bedding. I care about those who live in substandard housing and I care that substandard housing is so rampant.

In many cultures females eat after the males of the family have eaten. Foods are often withheld from females and given to males since they are considered most important to family survival. There are general nutritional deficiencies related to 
economic status, food availability, social prescriptions and proscriptions which directly and negatively affect childbearing, especially the intrapartal process.

Psychologically females have many lessons to learn about their future roles and functions. They are well prepared, through the socializations process, to be adaptable and pliable (Dollar \& Gatti, 1999; Levine, 2000a, 2000b; Levine, 2001; Moser, 1989; Staunton, 1990). For example, I remember one eight year old girl who had been "leased out" to a family to work as a maid. A monthly stipend was sent back to her family of origin so that they would have money to augment their meagre income. She worked 12-15 hour days, did not attend school, and slept on the floor in the kitchen, all this in preparation for her future vocations as wife/maid/mother.

The events associated with the childbearing process have direct effects on the family unit, the community (Aye, Champagne, \& Contandriopoulos, 2002) and the nation at large. Poverty encompasses every facet of these women's lives.

Access to health care in the developing nations is an interesting process not commonly known in the developed world. Hospitals and clinics can be geographically inaccessible as there are few, or no roads, nor public transportation Infrastructure, as one knows it in the developed world, does not exist. For those who have access to community clinics and/or hospitals, general care is available. Specialized care that may be needed is seldom if ever available (Grown et al. 2005; Tinker, 2000). Health care tends to be sought when birth is imminent which can negatively impact outcomes.

There is also the issue of social mores as pregnancy is considered a continuing fact of life and the discomforts that may be associated with it are not considered a reason to not fulfil one's usual role expectations. Another factor can be the negative consequences of accessing health because meal preparation for the family will not be done, household tasks will not be completed, and the kitchen garden, farm, or animals will not be tended to during the time it takes for the woman to travel to, wait her turn, receive health care and perhaps be hospitalised. I care about health care.

In developing countries, women are very much part of a silent, unseen economic backbone of the family unit (Beneria \& Bisnath, 1996; Browner \& Leslie, 
1996; Cattell, 1996; Cheru, 1992; Dollar \& Gatti, 1999; Enloe, 1990; Jiggins, 1989; Massiah, 1989; Meleis \& Lindgren, 2001; Pick, Ross \& Dada, 2002; Whiteford, 1996). Because childbearing is a constant factor in women's lives, women must continue with all their responsibilities during their pregnancies. Symptoms that may herald a catastrophic event in pregnancy are often ignored until morbidity and/or mortality are unavoidable. As mentioned previously it is usually when a pregnant woman is fully engaged in the labour process that she will access health care, be it the local TBA, government hospital, or outlying clinic (Cook \& Dickens, 2001; Mariko, 2003). Despite serious inequities in the provision of health care and minimal access, poor women seek and obtain health care in very traditional ways (Cook, Dickens, \& Syed, 2004).

I find great difficulty observing wilful maltreatment of human beings. I have often wondered if the reasons have to do with a person's need to feel superior to another. I have also reflected about health care professionals positioning themselves as the ultimate authority figures. I wonder, too, if part of the reason is the recipient of care is a woman, and/or a poor woman who is a non member of the majority culture. I envision, perhaps a utopian vision, a partnership between patient, family, and care provider regardless of financial status, ethnicity, race, religion, or gender preference.

Many organisations that have an international audience address protection of human rights, ensuring social justice and providing dignity and respect. The great religions of the world profess to do the same; yet, on a daily basis what is professed and what is practiced are quite different. Why is it that people are still treated with inequities? "The term human rights covers the rights possessed by all individuals by virtue of being human, "it represents the legal recognition of human dignity and equality among all individuals” (Bouchet-Saulnier, 2002, p. 125). Yet, to illustrate human inequality "requires examining factors like geography, gender, race and ethnicity, as well as economics” (Gershman \& Irwin, 2000, p. 16). Disparities exist, seventy percent of people living in absolute poverty are female, and also indigenous peoples experience excessive poverty (Buvinic, Gwin \& Bates, 1996). "Poverty, inequality, and patterns of economic change directly and indirectly shape health 
policy and health outcomes” (Gershman \& Irwin, 2000, p.11) and community and national functioning. Is it possible for one person to make a difference? Can I justify my going to another culture on the basis of our shared humanness? Can I provide dignity and respect internationally? I think that I can. I care.

For me, it is my connection to people that just “turns me on” and my desire to do the same for my students. I feel that students must be exposed to the world at large so that they will experience many different ways of living “one’s” life. Nursing students must begin to reflect upon the uniqueness of individuals, and their roles and functions within their social milieu. Students must learn to value and understand other than themselves and their society. I am endeavouring to educate students experientially so that as the world "shrinks", an individual's world has the immense capacity to expand. Experiential learning is the perfect vehicle as one can utilise all one's senses and cognition. It is also the potential to build professional practices to new visions and enhance understanding in personal social intercourse and beyond.

My inner voice and I are in constant communication. As Plato could have said, (Hikins, 1989, p. 28) “...thinking is an inner dialogue carried on by the mind and self”. In human interaction are we not constantly “dialoguing to ourselves” silently? This is akin to doing Kegel exercises "out in the open" but nobody but the self, knows what is going on. This inner dialogue can represent reflection, exposure, revelation and change. For me my "self dialogue" is forever re-visioning itself as I reflect and re-experience, through all my senses: events of myriad labours, deliveries, the vagaries of life, the rampant poverty and the stylised interactions of individuals and groups of people. Roberts, Edwards, and Barker (1987) described intrapersonal communication thusly: "the physiological and psychological processing of messages that happens within individuals at conscious and nonconscious levels as they attempt to understand themselves and their environment” (p. 2), as I am always endeavouring to do.

To intuitively understand that which is implicit, to internalise this process and use this personal "vital data base” in my life is a continuous pursuit. My memories take me back to my older daughter (Sioux Indian) and an early afternoon, shortly after returning from a walk in the hills, which was one of our daily rituals. I 
was getting ready for work and Joey turned on the television. She became engrossed in a traditional “cowboy and Indian” film. The United States Cavalry had just arrived and were beginning to slaughter the Indians, and rescue the "trekkers west", new settlers, who had set up a circle of Conestoga (covered) wagons to protect themselves. Joey, rushed to me, and said, “mum, mum, why are the Indians always bad?” What do you say to a four year old? Do you recite the litany, "stories” of history, manifest destiny, colonisation, racism, ethnocentrism, genocide, and imperialism? Or, do you just say what a child of that age can understand, hoping that she will not internalise the negativity perpetrated about her race? The mass media can have startling and unsettling effects upon individuals and groups whether portrayed unwittingly or in a subtle manner for nefarious purposes. When others are devalued I feel as if my own cultural boundaries have been trespassed.

When I volunteered at a Mother Teresa orphanage in New Delhi, India, I worked with one nun and one aide whose responsibilities included 50 abandoned babies. Physiological care was all that could be offered; bottles were propped, rags (no nappies here) were changed whenever time allowed, which wasn't often. By the time these babies were six months of age, through lack of stimulation, they would continually turn their heads from side to side. On more than one occasions one of the toddlers from an adjacent room would visit with us, and play with the roaches. One day, he managed to eat one, before personnel could get to him. When I share my stories with people, they always reply: this must be the exception, no I say, this is the rule.

At this time I was living with a very nice local family. I was denied access to the kitchen, even for a glass of water (the same glass was always saved for me), and understood implicitly that it was who I wasn't (Hindu) rather than who I was. I was perceived to have the potential to “contaminate”. In my own country it was the diametric opposite; who I was, was the core issue. Whilst an undergraduate student I was visiting the family of my roommate in rural central Florida. One afternoon we went out with a group of her friends, I said something, a common New York City phrase. The car literally came to a grinding halt: “Get out, you Jew”. My memory was immediately jolted back to a conversation with my father a year earlier as I was 
about to embark on my tertiary education. You will be in places, meet people, and hear things that are derogatory to Jewish people and others. Always remember your humanity, he said, and try to reach out.

As a young child our family took a weekend trip to Pennsylvania. We stopped for lunch in a small town restaurant. We waited and waited, the waitress tended to customers at hand and those just entering. It was as if we were invisible. My father finally said: we need to leave, as we will never be served here. As we drove through town we saw a sign and I remember it vividly, it said: "no Jews, no colored, no dogs"; a non-verbal assault, with a clear message, one that should shame all humankind. We live in a scary world today, where we have "significantly progressed” and violence is the norm. I pose a rhetorical question: Can violence be deconstructed through individual bridging, reaching across cultures, and understanding differences?

I implicitly know that to build trust one must reach out and thus my opening and concluding paragraphs of my presentations, in countries where English is not commonly spoken, is always in the national language(s). I work hard to accomplish this, enlist help from native speakers, and practice "forever". I have done this in Croatian, Finnish, German, Mandarin, Russian, Slovene, and Swedish. I take risks, for I know that I can easily be misunderstood and/or not understood. Audiences laugh, barriers come down, and basic humanness is shared, trust is being built.

Some years ago I gave a slide presentation/discussion in Munich to hospital personnel. My topic was, per usual, childbearing women in the developing world. During the presentation, and at the conclusion, there were so many audible sighs of disbelief and multiple queries. All were very familiar with the appalling statistics yet when individuals were contextualised and personalised in their daily lives, it really changed the focus from numbers to individual misery. The audience talked about their families, and they realised, truly, the vast differences in human existence.

When I was a nursing student the dictum was: do not get attached to patients. How can a nurse begin to build trust without a relationship? How can a nurse empower a patient without acceptance, listening, advocating, connecting, understanding, and therapeutically communicating? The potential of the 
nurse/patient relationship is enormous and deeply affects the psychosocial outcomes for the patients and her family. I was 40 years of age when I undertook my midwifery education in England. I was constantly told by the midwife in charge of the postnatal unit, don't linger with the patients; get your work done. Well, that is my work, building a relationship is extremely important, to both the patient and the midwife.

In pursuit of my epiphany I think of Kierkegaard (1851/trans. 1941) who said: “The difference between pursuit of career goal and ethico-religious commitment is that, to an even greater degree, all of life is included in the commitment, and one has oneself as the task” (p. 422). This means to me that my professional life and my avocation are bound into one. My life's commitment is to my work in the developing world that includes the "opening” of students' lives to the possibility of transformation. The transformation goes beyond nursing, affecting the very essence of life. Compassion, understanding, acceptance, tolerance, dignity and social support can clearly be explored. It can be modelled, can promote change and alter the world bit by bit. In stepping out into the world, humanity is honoured, the humanity of us all. It happens by promoting the connection of people to one another by sharing lives and commonalities. The following quotation reflects what I feel:

It is fairly certain that if the next generations do not know and understand the world much better than the present generation and its leaders, and opinion-makers, their survival chances will be in grave jeopardy. The locus of human co-operation for mutual enrichment as well as survival is moving from the national to the world level. (Singh, 1991, p. 60)

I am compelled to provide the opportunity for students to be exposed to the diversity of human life in all its richness. I also want them to work in the developing world with poor childbearing women, a particularly marginalised population (Catell, 1996; Grown et al. 2005;). In this era of "globalism” it is imperative to deepen the depth and breadth of lebenswelt, Husserl's (1913/trans. 1969) concept of "lived experience". The concept implies that which we really can understand and scrutinise in our daily existence regardless of where we are. Scrutiny promotes psychosocial 
and cognitive growth and the capacity to incorporate new lexicons into our daily lived lives.

I am of the privileged world, yet my world view takes me beyond the physiologic and psychosocial boundaries of the world of the "haves"” to the world of the have-nots, because I have been willing to move outside of it. My multiple immersions into the everyday lived lives of those who exist in poverty, is a world where one endeavours to make it through one day at a time by obtaining the basics of food, water and shelter. I would be remiss if I did not provide this for students, a world view altered, changed forever, each student's life profoundly affected which in turn influences the professional practice and personal life of each participant.

Clark Moustakas (1967) would say: "Experience is real only when it is being lived...” (p. 1), that implies the savouring and reflecting upon the daily tenor of life with all its shades and tones. For me this has paved the way to a lifestyle that does not include the cult of acquisition; I am content without the materialism that surrounds many people of the industrialised world. I often think of this anonymous quote: "Happiness is not having what you want but wanting what you have”.

I do not think of myself as a spiritual or religious person; I do think of myself as a humanist and a realist. My belief is that we all inhabit and share this planet despite its unequal distribution of resources such as health care, food, water and land distribution. In the psychosocial realm it is the unequal distribution of dignity, justice and basic human rights. For the majority of people in the developing world life is based on surviving rather than obtaining material wealth and possessions.

In many societies in the "south" men are more valued than women (Ahmed et al. 2000; Okojie, 1994; Pillai \& Gupta, 2006,) as evidenced by the acceptance of the inevitability of maternal/neonatal/infant morbidity and mortality. Poor women's roles and functions are bound up in domesticity and they are socialised to be submissive wives and daughters-in-law. Reproduction is also an important facet of their existence in order to insure economic survival for the family and for parents in their old age.

Moustakas (1977) wrote of “turning points” (p. 1) that affected his very essence of being and resulted in profound change in his life. Moustakas (1977) 
defined these turning points as “awareness of critical shifts in...growth” (p. 1) and also stated "What situation or realization precipitated the journey that made possible the discovery of who I am?” (p. 1). For me this critical junction came with my very first immersion experience into the developing world (1971). The acceptance, the understanding, the instant camaraderie of open and trusting villagers, my humanness, all came together and I realised that we as human beings share multiple commonalities.

I experienced this transformation in Africa, radically changing who I perceived myself to be. I stood helplessly by a neonate and his parents as the baby's life slowly and painfully ebbed from him due to neonatal tetanus. I assisted in the removal of a hip pin from a recently deceased elderly nun. The tools of my trade were a dull surgical knife and a pair of scissors. This took place in the sister's room.

I prepared “formula” for approximately 60 infants, patients in the paediatric unit, who had contracted the measles. Many of these infants were already moribund due to their debilitated state before contracting the disease. I nursed children and adults with smallpox who had received a vaccine that was too potent. I watched as village children slowly starved to death. I tended pregnant and labouring women with the chancres of syphilis. I watched women work 18 hours a day, without benefit of any modern conveniences, so that they and their children could survive to face the onslaught of the next day. I surgically incised breast abscesses and inserted wicks for many mothers who developed these abscesses due to their living conditions. These experiences profoundly affected me FOREVER. The dialectic of the world of the haves' and the have-nots became a reality as I immersed myself into the life of the hospital and all the people it served in the many surrounding communities.

The culture shock of returning to the over-developed world with its waste, pursuit of materialism, and the stark reality of the differences of the haves and havenots, is overwhelming. The harshness of life, the acceptance of the deaths of young women, babies and children and the ultimate pain of the death of one or more of one's children, were common African phenomena. I befriended whole families with their pot bellied young children, most with oedematous legs and golden red yellow 
hair. All this by products of kwashiorkor, were common and part of daily life. Of the under five children on the measles ward, many had Noma which is a gangrenous infection of the mouth that ultimately took the life of the child.

I am consistently guided by feelings. I am intuitive and can readily understand and empathise with others; I have never considered myself "a thinker”. It is people and who they are and what they share of themselves that really have meaning for me. I am constantly learning from and about others.

My perceptions have also changed since beginning my $\mathrm{PhD}$ studies in New Zealand. I have begun to realise that as I enter a culture outside of my own, I will never truly comprehend it in its entirety. Even within my own cultural bearings, I cannot ever understand or know all there is to know (Linton, 1955). Each individual's uniqueness is shaped by time and space and life experience, ad infinitum. Each individual's uniqueness is also predicated on sociological institutions, individual family beliefs, and the socialisation process including the stimuli that surrounds and interacts with each of us. One can never truly know another's "self talk" and what truly propels each to think and act in certain ways (Ramsden, 1990, 1996, 2002). Regardless, not treating people with respect and dignity no matter who they are and what they believe is unacceptable to me.

I take my “cues” from my patients and their families, in my interactions, as to what is "okay". My psychosocial connections have always been my strength. The trust, the cooperation and my intuitive knowing of boundaries not to be crossed and perhaps the most important issues, my reaching out, my caring, have enabled me to be effective. This, along with my flexibility, adaptability and development of mutually trusting relationships with my patients and their families has been my continuing source of contentment, delight, gratification and satisfaction that I feel as a health care provider.

Each of us "lugs" old "baggage", and personally this means my need to be involved and connected with other people, a need to look at society and health care issues and a need to address inequities. "Every baggage room of memories is open day and night, for no living traveller rides free of the burden of old luggage” (Ross, 
1991, p. 7). My inner voice continually plays the songs and melodies of my youth, of my family, and all are related to justice, equality, dignity and caring.

I am "haunted" by psychological and/or the sociologic pain of individuals and groups, by useless suffering that I have seen in my professional and personal life. Perhaps it is growing up in a society where one is different than the majority that intuitively lends potential understanding of another. Perhaps I internalised the psychosocial feelings of having a world minority status.

I am one individual and each patient entrusted to my practice is cared for with honour and respect. I would not be true to myself if I did any differently, and I could not. It's greater than compromising one's standards of care: its devaluing life itself. I continually search, endeavouring to find my way toward truth in the world. This is a lifetime journey on a long and circuitous route. I know that it is imperative for me to expose students to other worlds; students learn that one's personal reality is not universal.

My way of knowing is deeply rooted in who I am and it is my guiding light on how I conduct my life. I reflect on my actions and examine them for meaningfulness as a human sojourner. I constantly have flashbacks and “discussions” with my inner voice about my work and my students’ work in the developing world. It is the realisation that there are parallels, likenesses and connections in disparities, that there are universal feelings, yet emotions are played out and finely honed according to one's culture. Culture can mean many things, an individual, a family unit, a group, a community, a nation, a philosophy. It is the exposure to every day lived lives that brings an understanding and growth process to the multiple concerns that all humanity share.

I think about how these journeys have dispelled complacency as one is immersed into the social fabric and the health care system of others. I think about the realisation of the power that societal institutions have upon us daily. I think about how I have put more than one professional position on the line when my advocacy role superseded my job description for this is who I am. McEldowney (2003) addressed this as “crossing the boundary, laying oneself on the line” (p. 171) and "walk[ing] the talk" (p. 199). 
These incidents took place in both the developing and developed world. I vividly remember having my hand slapped by a passing obstetrician as I was holding the hand of a woman in labour and talking to her. I was told, "we don't do that here” (name of country omitted). In another country a doctor denounced the labouring woman I was working with because of her sexually transmitted disease (lymphogranuloma venerium), which was extremely disfiguring to her vulva, causing her excruciating pain. He said she was just “poor trash”, but I knew this woman having befriended her weeks before. She had no control over any aspect of her life, and it was her husband who was having extra marital affairs who had given her the disease.

When I worked as a public health nurse in New York City I accompanied a young, poor, non English speaking mother and her seven children on public transport in order for the children to receive their immunisations. My supervisor informed that that this wouldn't be tolerated as it was the mother's problem, not mine, actually, I felt it was and continues to be a societal problem.

There are those who are motivated by religious doctrines to reach out; I am motivated by my humanness and by the socialisation process that I internalised and is lived by my extended family and myself “... since to know the world is profoundly to be in the world in a certain way...” (Van Manen,1990, p. 5). For me being is travelling the world and attempting to know it. I am what I am and experience the world primarily through my own eyes. My focus: human connectedness, caring and reaching out.

I recently read W.H. Auden's (as cited in Van Manen, 1990) definition of person. “...person refers to the uniqueness of each human being... as persons, we are incomparable, unclassifiable, uncountable, irreplaceable” (p. 6). It was fascinating to find this quote as it totally encompasses my position as to the essence of the individuality of every human being. To me, travelling and immersing myself in others' cultures reinforces the beauty of learning about persons, families and communities. Each is so different, yet belongs to the human family.

Gadamer (1996/trans. 2000, Weinsheimer, 1985) addressed lived experiences as related to each other, such as developing world, life lived, poverty, 
childbearing women, caring and health care. These may seem as isolated units but they are really connected as a whole. For the students, by using the medium of the developing world in its entirety, the lived experience became part of each student's lexicon of life experience as a totality. Each student was able to fully engage in the immersion experience, and adapt her/his modus operandi to reflect that individual's unique lived experience. In so doing each broadened her/his own world view. According to Van Manen (1990) “...we tend to forget that the change we aim for may have different significance for different persons” (p. 7). As each of us is an individual with our own life's baggage and culture, that which we get out of an experience is thus very individualised.

My style of teaching is directly related to who I am and "what I do is me”, as expressed in G. Manley Hopkins’ poem “As Kingfishers Catch Fire” (1918/1948, p. 95). “...The broadest possible set of experiences is crucial to help each understand....as completely as possible” (Takacs, 2003, p. 28). I respect and relish differences which enables me to more fully understand an ever expanding world view and, by extension, the world at large; "diverse perspectives enable us to experience the world more richly and come to know ourselves more deeply” (Takacs, p. 29). “Knowledge comes in part through life experience, all can learn-but only if you listen” (Takacs, p. 32). By hearing the perspectives of others you "may question your assumptions” (Takacs, p. 32) and “...become more aware of who we [you] are by communicating with those who are not like us [you]” (Thayer-Bacon, 2000, p. 104) thereby opening yourself to the world of others. "Schooling is not preparation for life: it is life” (Bevis \& Murray, 1990; p. 328); I teach to this fact. “...Teaching is built on relationships, connecting students' learning experiences into their life experiences” (Wood, C., 2002b, p. 196) and the teacher connecting with the student (Ambrose \& Bridges, 2005; Barlow, 2005; Cangelosi, 2005; Clark, 2005; Freire, 1998; hooks, 2003; Paterson, 1991; Reavy, 2003; Scanlan, 1996). “...How a teacher makes human connections are the most dramatic influences on student learning” (Jackson \& Jackson, 2002. p. 287). I bring “myself authentically into the classroom and into the lives of my students while still maintaining an appropriate professional” (Kunzman, 2002, pp. 89-90) standard. 
Each of my students is a unique individual and I respect their beliefs and values as I develop a relationship that promotes learning through understanding the individual and the group praxis. "When we go into the classroom without passion, without heart, we are denying our students....We are denying our students ourselves” (Henningfeld, 2004, p. 54). My students and I develop a relationship that is a journey of “our learning, reflection and growth” (Tanner, 2004, p. 4).

We embark on a joint journey, and “develop a sense of community and responsibility for fellow humans” (Bevis \& Murray, 1990, p. 330). We address the institutions of society and we study the relationship upon individuals, groups, and students. By expressing feelings, the potential exists for personal and group growth, learning, and transformation, the latter occurring "in far from equilibrium states through disruption at the intersection between order and chaos” (Newman, 2003, p. 240). The "learning process must be reimbued with the texture and feeling of human experiences shared and interpreted through dialogue with one another” (Kolb, 1984, p. 2).

Why do I take students on journeys that students say changes their lives? Perhaps it is a selfish need, my humanistic, idealised vision of how the world should be. My belief is that every human being should be treated with dignity; that every human being has an intrinsic worth; and that every human being holds a place of importance in her/his milieu and perhaps beyond. Perhaps it is a vested interest, my personal vision of expanding one's world view; perhaps it harkens back to who I am...I must do this, I am programmed to do this...I must expose students to the world at large, other. It is my commitment and a moral imperative to me. It is my own personal moral outrage at a world that is "blind" to the suffering of others. This is the 21st century and still

reproductive health is closely linked to the global burden of illness, poverty, and loss of economic productivity. Those most affected are the 1 [one] billon women of childbearing age who live in poverty and another 1 [one] billon adolescents on the cusp of adulthood (Anderson, B., 2005, p. 352);

one-third of the world's population.

At the beginning of these journeys, I never know what the outcome will be as each individual embarks on an expedition of exposure, immersion, and reflection. 
Each student is unique and has her/his value system that affects her/his individual outcome and gives credence to multiple realities, yet there are common themes that supersede the individual ones.

Having taken seven groups of students to work internationally I can truly see the fruits of these ventures. Experiential learning has meant personal growth that endorses, supports, advances, and encourages a sustainable change, a different outlook on life both professionally and personally. I am an idealistic person and fervently believe the greater the knowledge of the "isms", and knowing about the power structures, the health care systems, the economics, family, religion and government, the more likely a metamorphosis. The importance of the human connection is a vital link. I hearken to my belief in the parallels that different countries share similar goals yet the method of attainment and problem solving are vastly different.

I recently read an article, "Faith, Hope and Clarity” (B. Carter, 2004) about a quest for religion/spirituality and one of the persons that the author interviewed stated: "it's about the journey and not about the answers; it's all about the tools and the resources” (p. 59). I, as well as the students, are on a journey toward enlightenment using flexibility, adaptability, and reflexivity. Although answers may evolve, on and beyond the "voyage" it is even more important to continue on the “expedition”. This is a powerful lifetime commitment to learning, discovery, and change.

In "teaching for and with social change", the subject of McEldowney's (2003, p. 83) doctoral thesis, the author states "I show that who the participants are as people is an intrinsic part of what they do as shape-shifters” (p. 83). The term "shape-shifters" is metaphorical and represents, for McEldowney, a construct for understanding teaching for social change. All of the participants "had moments in which they challenged the status quo” (p. 83) and recounted their memories of being different. I can readily identify with these two concepts and perhaps somehow I fit into this model. Is it possible that I have been a shape-shifter without the realisation of what I have been doing? 


\section{Summary}

This chapter has covered all the facets of my epiphany leading to my discovery of whom I am and why I do what I do. The next chapter explores the literature on international immersion programmes for baccalaureate nursing students in order to learn what other research projects and immersion programmes cite as outcomes. In this way, I could then look at my work for its contribution to this body of knowledge. 


\title{
CHAPTER IX
}

\section{REFLECTIONS ON THE EXPERIENCES OF OTHERS: A REVIEW OF THE LITERATURE}

\author{
"Whether our work is art or science or the daily \\ work of society, it is only the form in which we \\ explore our experience which is different; the \\ need to explore remains the same"
}

(Bronowski, 1965, p.72).

\section{Introduction}

My journey of self discovery has led me to review the literature on international programmes for nursing students in order to find out, understand, and learn what others have experienced and how international immersion experiences have been perceived by both students and faculty. The international immersion programmes have been a strategic focal point of my personal and professional life for many years. Also, the literature on international immersion programmes was reviewed and comparisons made to see if similarities or differences in research findings existed.

\section{Literature Review}

The majority of the international immersion articles were anecdotal and descriptive in nature. Others were more formal studies. Forty-five international programme reports published between 1984 and 2005 have been included in this review (Table 9.1). The programmes that I have drawn on were analysed for purpose, characteristics of the programme, venues, accompaniment by faculty, preparation for the student experience, and identified outcomes. The initiating countries for the programmes were Australia, England, Finland, Holland, Northern Ireland, Spain, Sweden, and the USA. The majority of the programmes involved immersion in first world countries as opposed to the developing world. 
During this period McAuliffe and Cohen (2005) conducted a review of the literature on international nursing educational exchanges and found that "most [articles] were written by faculty, even though most exchanges were for students” (p. 21).

Studies described students who negotiated their own solo experiences (Pross 2000, 2003) to students on highly organised and scheduled journeys (DeDee \& Stewart, 2004; Colling \& Wilson, 1998; Inglis, Rolls \& Kristy, 2000, 1998; Rolls, Inglis \& Kristy, 1997; Lee, N., 1997). The purpose of the studies is the first aspect that will be discussed.

\section{Purpose}

An umbrella term, “personal and professional growth” was used to describe the purposes for initiating most international programmes for student nurses. Evanson and Zust (2004) conducted a qualitative study using content analysis of journals, participant observation, narratives, and focus groups and sought to describe the meaning of participation in an international immersion experience. The programme took place in Guatemala and lasted ten days. They found students experienced “personal growth”, described as having a greater understanding of self and relationships, as well as stimulating professional changes. Professional changes focused on understanding the effects of social injustice on people's lives and “appreciating the whole person in their care”, (Evanson \& Zust, p. 10) according to the research team. The researchers (Evanson \& Zust) concluded that there needs to be ongoing research to validate growth and address the long term impact.

Callister (2004) conducted a phenomenological study to assess the long term impact on former nursing students who had participated in international elective immersion programmes from 1995-2002. Callister found that the "over-riding theme was gaining a global perspective, which included themes such as increased understanding of other cultures and people, gaining an understanding of global health issues, experiencing personal growth, making interpersonal connections, and increased cultural competence” (p. 361). 
Purnell (1998), a narrative descriptive account, took a group of students to Panama for three weeks in order for the participants "to grow” by experiencing another culture. It was conjectured the students could learn about differences in lifestyles and health care delivery that would then enable personal growth. This was similar to what G. Stevens (1998) did in Nicaragua on a trip that lasted 10 days, and Lindquist (1984) in an exchange to England. Exposure was thought to allow growth simply by going to a different environment. Students experiencing life from the vantage point of others reported personal growth experiences in understanding others (G. Stevens, 1998), and in alternate forms of family structure and communication styles (Purnell, 1998).

Inglis et al. (1998, 2000) and Rolls et al. (1997) addressed both cognitive and attitudinal changes for students who were situated in placements in non industrialised nations. The Inglis et al. (2000) study utilised a questionnaire measuring changes in perception through content analysis and the Inglis et al. (1998) study was a pilot study utilising open ended interviews, looking at potential changes in students' conceptual understanding of community health nursing, based on hypothetical health related issues in a culture external to the students. The purpose was to see if there were shifts in understandings before, during and after participation in the programme. The 1997 study (Rolls et al.) was a nonexperimental descriptive study using interviews before, during and after the study abroad programme. The researchers Inglis et al. (1998, 2000), and Rolls et al. (1997) thought growth would be inevitable by removing students from their world and placing them in the world of others. In these two studies it was reported that students gained increased knowledge and understanding of cultures. Students had positive attitude changes toward the culture, religion and lifestyles of others. Inglis et al. (2000) stated "whether these changes were permanent or transitory cannot be determined from this study” (p. 254).

Ryan, Twibell, Brigham and Bennett (2000) had an ongoing programme with venues in both the developed and developing world and used a grounded theory focus to explore the effects of immersion programmes. The conclusions were that there should be "curriculum designed to integrate culture throughout the nursing 
program, as well as faculty who value and model cultural competence” (Ryan et al., 2000, p. 406) inferring that growth had indeed taken place. They found that because students participated in international programmes, they exhibited changes in their values such as increased insight, increased cultural sensitivity and the positive acceptance of others whose lifestyles are different, through becoming aware of their own biases. They also increased their abilities to communicate, as well as demonstrating increased flexibility and creativity (Ryan et al., 2000). Leh, Robb and Albin (2004) addressed an ongoing programme for students from the USA who had structured observational visits to various health care facilities in Sweden. They described the outcomes as fostering students’ global awareness and building personal confidence; the article was a narrative account of the programme. Holstege (2000) described an immersion programme in Nicaragua as students worked with people ravaged by a hurricane. Students expanded their views on world health issues and acknowledged differences in cultures as they challenged their own values and beliefs towards human inclusion, lifestyles, and world views. This study (Holstege, 2000) was phenomenological in approach and focused on the lived experience of the students addressing culture shock, adjustments, lessons learned, and memories.

Duffy, Harju, Huittinen \& Trayner (1999) discussed the placement of Finnish nursing students in the USA for a Public Health experience so that students could be immersed as "foreigners"; this was a descriptive account of the exchange programme. Results indicated that students were able to compare and contrast issues related to public health in both countries, “the first step in making changes” (Duffy et al., 1999, p. 30). Finnish students with good English skills developed teaching plans, taught health education classes and also counselled patients.

St.Clair and McKenry (1999) measured “culture competence” using a two group research design. There was both an experimental and control group; the latter did not participate in an international exchange programme. They found that "short term international nursing clinical immersion experiences” (St.Clair \& McKenry, p. 233) supported "cultural competence” versus the control group that worked with culturally diverse patients in their home communities. Students in their own communities did not experience any change in their ethnocentric status while 
students who went abroad did (St.Clair \& McKenry). Cultural competence in this study was defined as being able to practice nursing with individuals, groups, and communities essentially within the cultural framework of race, gender, and sexual orientation. St.Clair and McKenry further defined cultural competence as being able to nurse individuals with an understanding of their traditions and values.

Cotroneo, Grunzweig, and Hollingsworth (1986) took students to Austria and Yugoslavia for two weeks to compare, contrast, and expand their understanding of health care systems at home and abroad with the purpose of increasing intercultural competency and growth. They found students' growth and learning were positively and greatly influenced through international immersion programmes. This article (Cotroneo et al.) was a descriptive account of an international exchange for students nurses. Students reported that their knowledge of European health care systems increased and that they also "came to rethink their own American context" (Cotroneo et al., p. 385) in how they viewed American health care delivery.

Zorn, Ponick, and Peck (1995) had an ongoing programme in England for nursing students from the USA and used a quasi-experimental approach to measure student cognitive development. Cognitive development was defined as "personal and professional growth”. Their findings stated that students' cognitive development was considerably improved compared to students who did not partake in the immersion experience. Frisch (1990) designed a cohort study and was interested in cognitive growth of students from the USA. The students participated in an immersion programme in Mexico and the findings were comparable to Zorn et al. (1995). Zorn (1996) was one of the few researchers who addressed the long-term impact on students who participated in study abroad programmes. The venues were England, Denmark, Scotland, and Russia. The reported long term growth was directly related to a greater understanding of global issues, personal openness, and accepting differences in others not seen in non-immersed students (Zorn, 1996).

Thompson, Boore and Deeny (2000) wrote about ongoing programmes for nursing students in Northern Ireland that took place in both developed and developing nations for the purpose of students' broadening and intensifying their understanding of societal effects on life, health and health care. A questionnaire was 
used with a five point Likert type scale to evaluate differences between students’ experiences in developed versus developing countries and the impact of the immersion experience on personal and professional development. The results showed that "students' [achieved a greater] understanding of the influence of cultural factors on health and healthcare” (Thompson et al., 2000, p. 491). The study stated students "who had undertaken their international experience in developing countries had gained [more comprehensive] international perspectives, personal development and intellectual development” (Thompson et al., 2000, p. 489) than their counterparts who had participated in immersion programmes in industrialised nations. Graves (2003) described a programme of a one time academic and cultural exchange for American (USA) students with a department of nursing in Chile that lasted one week. The purpose was for the visiting students to learn about the role of Chilean community health nurses. The outcome was described by individual comments relating to “learning more about oneself” (Graves, 2003, p. 20) through international immersion, and becoming more sensitive to language and life styles. Students also felt that they needed a greater time frame for the immersion, a theme found in several very short-term projects (Cedar Crest hosts, 1996; Sandin, Grahn, \& Kronvall, 2004).

Even though the expressed purposes of a number of the studies differed semantically, all 45 studies coalesced to one outstanding feature. The characteristic common to all the studies was looking for a change in individuals' perceptions, both in the personal and professional realms through participation in international programmes. The next issue to be addressed is characteristics of the respective programmes.

\section{Programme characteristics (venue and accompaniment)}

There were two issues of importance specific to programme characteristics. It seemed important to know whether students were accompanied or unaccompanied by faculty plus discern if the students were strictly observing/touring institutions or directly offering hands-on patient care. The continuing presence, support, participation, and interaction by faculty from the home school, according to the 
research, had the potential to enormously impact participants. In many studies it was difficult to ascertain if faculty actually did accompany students (Ailinger \& Carty, 1996; Bond \& Jones, 1994, 1993; Cedar Crest hosts, 1996; De Dee \& Stewart, 2003; Kollar \& Ailinger, 2002, 1996; N. Lee, 2004). There were studies undertaken by faculty who did not participate in international programmes but had an interest in the subject and who conducted interviews and utilised questionnaires that addressed outcomes following the international experience. For instance, Pross $(2000,2003)$ conducted a phenomenological study of lived experiences of students through a post immersion mail survey and interviews. The findings were divided into four categories that measured preparation, adjustment, caring, and transformation. Pross (2000, 2003) felt that the individual values, and the culture of the students were directly affected by their participation. All students were not affected identically but in general the participants felt that they had all participated in a "once in a lifetime" opportunity, gaining knowledge in global understanding that challenged their world view. All the participants experienced culture shock, defined in this study as "any physical or emotional discomfort experience[d] by those adjusting to a new environment” (Pross, 2000, p. 40) as they moved from “one world to another”. One student defined culture shock in the following way, “you can't imagine the vastness of the poverty because you just don't ever see it here [USA]. There is no way to prepare for this” (Pross, 2003, p. 399). Pross (2000, 2003) concluded that long term longitudinal studies are necessary to truly determine the real outcomes of immersion programmes.

Callister (2004), and Callister and Hobbins-Garbett (2000) described an international service learning programme where faculty accompany and "role model to the students cultural sensitivity related to health issues” (p. 181) at the venue in the developing world. Students were perceived to be learning "social responsibility" as integral to their emerging professional nursing role. St. Clair and McKenry (1999) utilised a triangulated research design with senior nursing students to assess perceived changes in cultural competence for those who participated in short term (two to three week) international exchanges. It is not known if the students were accompanied on all or any of the international segments. The findings demonstrated 
that being a "cultural minority” in an international setting enabled students to “...recognize their 'unknown ethnocentrism”” (St. Clair and McKenry, 1999, p. 234).

Another common denominator for several programmes was the venue, developing countries and whether care was observed or actually given by the nursing students. Cummings (1998) described an on going (hands-on) international exchange of four weeks duration, the venue was Barbados. Students were accompanied by a faculty person from their home university. The accompaniment resulted in faculty attending all presentations, lectures, and tours of facilities with the students. The faculty person was also instrumental in setting up the clinical rotations with the host hospital matron and nursing staff. Students worked with preceptors on all the units of the hospital providing complete hands-on nursing care whilst the accompanying professor rotated among the hospital units which provided students with continuing support from their home school nursing professor. Fennal, Fair, Fleming, Gibson, Hugee, and Winborne-Tanner (1998), Hadwiger and Hadwiger (1998), and Peel (1998) also described programmes where students were accompanied by home university faculty and were thoroughly engaged, fully participating in the provision of intense hands-on nursing care and students felt that they were truly part of the health care team. Tabi and Mukherjee (2003) and Walsh and De Joseph (2003) had similar programmes with students providing extensive hands-on nursing care. This resulted in students having a facilitator "on board” to support them throughout their immersion experience.

The next set of studies described programmes where students were not accompanied to the venue by home university faculty (Lee, 2004; Sandin, et al., 2004; Watt, Law, Ots \& Waagø, 2002). Students were sent abroad to immersion sites with varying degrees of preparation and participation for the hands-on experience. Sandin et al. (2004) stated that the "students need[ed] to have more knowledge about the culture to be encountered” (p. 230) so that they would have the appropriate knowledge (tools) about how to interact with the hospital staff and patients and feel more comfortable in their socio/cultural communications. Students also appeared to be unsure of their clinical role and their interactions with hospital 
personnel. The authors (Sandin et al., 2004) also stated that both individual and group discussions among the participants should take place during the immersion as well as "constant dialogue between students and teacher is important to develop understanding of one's own values, beliefs, and behavioural patterns” (p. 230).

Yonge (1997) stated that students without the presence of faculty "have to negotiate learning opportunities, manage conflicts and stress” (p. 815) which attenuates the entire immersion experience Grant and McKenna (2003) reported in their descriptive exploratory approach of Australian students’ four week clinical immersion in Great Britain: "One of the roles of a clinical teacher is to support students through difficult experiences and assist with debriefing afterwards... On international clinical placements students may not have the same resources for clinical teaching, supervision or debriefing” (p. 534). Because of the cultural components, students are in special need of support and guidance. Grant and McKenna (2003) stated the importance of students being “able to access...clinical or university staff” (p.534) in order to have a support system to resolve potential conflicts and concerns.

Watt et al. (2002) wrote about international exchange programmes for university students that were organised through the European Socrates-Erasmus programmes. These are ongoing programmes among many European countries and supported by government grants. The foci of the programmes are "people to people" relationship establishment that includes language, personal and professional development, and immersion into a foreign country. For student nurses the programmes provide international student exchanges consisting of observational experience in a clinical setting as well as social and cultural outings. The students, however, were not accompanied by their home school faculty. Nursing students from 26 colleges located in 14 European countries went abroad in this programme. Results showed “considerable cultural learning can occur” (Watt et al., p. 323) with facilitated exchange experiences.

The case study by N. Lee, (2004) was conducted in a school of nursing in England that involved student nurses participating in an immersion programme outside of the United Kingdom, venues were not given. Preparation included course 
tutors who conducted a literature review focusing on international studies for undergraduate nursing students citing the possible benefits of participation. For students, the preparation consisted of a semester long module entitled "International Nursing and Health Care”. The expectations were that each of the students had to organise their own immersion segment. The results revealed that the international “experience was more demanding then they [students] or facilitators [tutors] had originally expected” (N. Lee, p. 118). Student comments included considerable social isolation and a nursing practise that differed from that of their own country. Tutor comments contained statements that addressed the importance of experiential learning versus classroom learning and the potential negative influence of the “facilitator’s own prejudices and stereotypes” (N. Lee, p. 117). This is important as students have the potential of beginning their journeys with preconceived, misconstrued ideas about people, practices, and institutions. This then colours the students' experiences. The results did state that the most significant learning for students was personal growth and "the examination of nursing practices [observations in host country] and the impact of feeling different” (N. Lee, p. 120) from the people in the immersion country. Faculty from the participating schools were unknown to the students on the exchange. Because the students were working in a country and health care system that were foreign to them they experienced personal and professional distress.

Scholes and Moore (2000) reported on an ongoing programme for nursing students from three collaborating nursing programmes in England, Holland, and Spain. For the immersion portion of the experience, faculty at the immersion site served as preceptors, and were previously unknown to the students which in itself became an intrinsic part of the international learning experience. The faculty of the three involved schools attempted to share modules to prepare their students. This proved to be difficult as each country had its own standards, procedures, and rules that guided their own nursing programmes and made crossover learning difficult. One of the prerequisites was studying a second language before partaking in the immersion segment but this did not help significantly either. At the venue institutions, students experienced “social dislocation which was profound and 
confusing” (Scholes \& Moore, p. 66) directly related to everyday hospital routines. At the same time, students learned what it was like for hospitalised patients who feel a perceived and real loss of control. Students did not have their usual support systems in place, nor have faculty from their home schools although they did have preceptors. The placements lasted three months and participants' outcomes included growth through comparing and contrasting differences in culture and health care practices; "students found their experiences on exchange diverse and powerful” (Scholes \& Moore, p. 69).

One ongoing USA programme (Currier, Omar, Talarzyk \& Guerrero, 2000) was a semester in length and took place in Mexico. Students had periodic visits from their home school professor, otherwise local faculty were the preceptors. The conclusions of the study were that students developed a "broad perspective of nursing and health care in Mexico and develop(ed) an appreciation for its language and culture” (Currier et al., p. 293).

Another factor of importance concerning programme characteristics was the issue of clinical tours/observations versus actual hands-on patient care. According to Fennal et al. (1998), one of the goals of the international immersion programme was for students to be able to understand the differences in the delivery of nursing care between the USA and the immersion country, the Dominican Republic. Students provided direct nursing care to clients on many hospital units. They understood that they could give quality care despite economic resource constraints.

A few of the studies cited involvement in public health projects. Although there was no direct hands-on care, students designed and implemented a health education project that directed their activity rather than being observers (Duffy et al., 1999; Inglis et al., 1998; Rolls, et al., 1997; G. Stevens, 1998). In several studies, there was considerable difficulty discerning if students participated in clinical care or not (Kollar \& Ailinger, 2002; N. Lee, 2004; Pross, 2003; Sandin et al., 2004). Leaving out this key element made assessment of each project more difficult.

Bond and Jones $(1993,1994)$ initiated a programme solely for culture and language immersion. It was felt that students who lived in a border (USA-Mexico) town knew very little about their many Mexican-American neighbours or their 
culture so they were immersed in a language and culture programme in Mexico. Students felt that the programme enabled them to increase their language skills and knowledge base about their clients. Faculty conclusions were that students gained “...skills and principles which will enable them to generalize to other cultural groups” (Bond \& Jones, 1994, p. 68).

Hands-on clinical experience was described in many of the studies including Cummings (1998), Fennal et al. (1998), Haloburdo and Thompson (1998), Peel (1998), and Walsh, (2003). The Haloburdo and Thompson (1998) study suggested the importance of including the opportunity for students to give direct nursing care. In this study comparisons were made between students who were immersed in developing nations and those in developed countries. The biggest difference found was that those students who were immersed in developing countries rekindled "their reconnection with caring as the essence of nursing” (Haloburdo \& Thompson, 1998, p. 19). Those students “accompanied by a faculty member, identified their presence [with clients] as contributing positively to the learning experience” (Haloburdo \& Thompson, 1998, p. 19) and the study also suggested the value of "providing direct client care, and [that] such experiences should be considered when planning international” (p. 19) immersion programmes. In addition Thompson et al. (2000) found that students who had participated in hands-on exchanges to developing countries had gained "significantly more in relation to international perspectives, personal development and intellectual development than those who visited developed countries” (Thompson et al. p. 489).

There were many variations in the programmes reviewed related to students being accompanied by faculty or venturing out on their own. The degree of participation in health care provision in the venue country demonstrated a long continuum from strictly observational to provision of full patient care.

\section{Preparation for immersion}

In the arena of preparation for international immersion, there were wide differences reflected in the literature. An important factor to consider is that student experiences can be shaped by the initial period of preparation, how orientation and 
direction are presented, and what is presented. Scholes and Moore (2000) reported a ten week preparation period for English students going to either Holland or Spain. The preparation included liberal arts, culture, and a transcultural framework to prepare nursing students for a three month period in either Holland or Spain, with the other involved countries doing the same. The aim was to produce culturally sensitive nurses by being immersed in another country's clinical environment. In the immersion segment, students worked with faculty from the country in which they were immersed and learned about differing styles and expectations of clinical tutors. Goldberg and Brancato (1998) reported a one day orientation for USA students participating in an exchange to Great Britain. The orientation included discussion of cultural similarities and differences, the travel itinerary, a slide presentation on health care in Great Britain, and a discussion about goals and expectations of the programme. Students participated in cultural tours, observations, classes and one day "in practice” with a British counterpart. The results were that students were able to compare and contrast the "strengths and weaknesses of their own country's healthcare in comparison to another country's healthcare system” (p. 34) and thus were more cognizant of worldwide issues affecting the nursing profession.

Sandin et al. (2004) reported a one week preparation for Swedish nursing students who were travelling to Tanzania. The preparations were focused on the hospital, the students' contact person at the hospital, clothes to wear, and what to bring. There was no mention of cultural or political preparation related to the venue. Students "attempted to understand the value and behavioural divergences they encountered, yet values of their home culture remained their frame of reference (Sandin et al., 2004, p. 225). Walsh (2003) reported mandatory attendance at planning meetings and utilised a service learning methodology for international immersion in Guatemala that included reflection, development of leadership, meaningful service, reciprocity, and hands-on nursing within the venue communities. Outcomes included students' intense understanding of the extent of abysmal poverty for families in the developing world versus their prior work in poverty areas in their own home communities. Students also talked about their 
increase in hand-on skills versus dependence on technology. They were especially struck by their own abilities to use their own physical assessment skills.

Pross (2000, 2003) reported that some of the participants in her phenomenological study did their own self-planning for their immersion experience, but other participants were involved in group preparation done by the students themselves such as investigating the municipal transportation system in the venue city. The experiences that students undertook were thus individualised and predicated on particular individual or group preparation. There were varying degrees of culture shock and students described being angry, afraid, frustrated, and having a fear of the unknown (Pross, 2000). The preparatory segment for Inglis et al. (2000) included “intensive pre-departure” (p. 249) orientation, however no details of content covered or time involved were included in the study. The results of the study stated that students were deeply troubled about the poverty of the people but students also became more independent in their professional nursing role in the face of poverty, a noteworthy finding. The authors (Inglis et al. 2000) pointed out that most immersion programmes reported in the literature are offered by the USA with student placements in Europe versus the majority of Australian study abroad placements that take place in Asia. The sites for the Inglis et al. $(1998,2000)$ and Rolls et al. (1997) were Nepal and Thailand as these countries are their "neighbours".

G. Stevens (1998) reported students were given a faculty-designed notebook with readings that described the venue country, government, and customs. Cummings (1998) reported “awareness” and "pre-planning” sessions “...to assist students to better understand the course expectations and the conditions to expect in Barbados” (Cummings, 1998, p. 43); this enabled students to immerse themselves more readily into the venue community.

Cotroneo et al. (1986) used an extensive reading list, had meetings with the students to discuss the trip, and had one seminar by an expert lecturer on Central and Eastern Europe, the site of the programme. The planning activities were carried out to enhance the experiences of the students by preparing them for participation in cultures that were different from their accustomed life patterns. Student participants 
felt that the preparation enabled them to have a comprehensive understanding of the goals of the programme, and helped them to make the transition to other cultures. There was no mention of preparation in several studies such as Anders (2001), Graves (2003), or Parnell (1998).

The reason preparation matters: students have expectations related to their roles, functions, goals and objectives from their own ethnocentric view. Their perspective is expanded as they learn about the requirements of the code of dress at work and at play, and the expectations about their conduct and other salient factors. Bonding with peers and instructor also takes place that results in a psychosocial support system. Preparation related to the life style, the government, education, religion, family, and economics of the country will give students greater insight, understanding, and a basis to use for contrasting and comparing lives. During the preparation period students also get to know more about themselves and how they may potentially react to differences. The organisational components of each programme were unique in preparing students for the international immersion segment. The continuum of preplanning progressed from nil to extensive and elaborate formats.

\section{Outcomes}

There was almost universal positive consensus about expectations and outcomes of international immersion programmes for nursing students. Students became aware of different health care delivery systems, how they operated, and how it affected the populace (Grant \& McKenna; 2003; Leh et al. 2004, Walsh, 2003). Students observed, toured, attended lectures, and participated in direct hands-on health care provision depending upon the programme they participated in (DeDee \& Stewart, 2003; Leh et al., 2004; Thompson et al., 2000; Watt et al., 2002). There were varying degrees of immersion into the host society (Colling \& Wilson, 1998; Duffy et al., 1999; Inglis et al., 1998, 2000; Sandin et al., 2004). As students’ cultural worlds expanded, students became aware that they "had a culture", their own (Button, Green, Tengnah, Johansson, \& Baker, 2005; Rolls et al., 1997; Ryan et al., 2000). They became aware of their ethnocentrism (Evanson \& Zust, 2004; Ryan 
et al., 2000; St. Clair \& McKenry, 1999). They clearly “grew” professionally and personally according to research reports (Currier et al., 2000; Haloburdo \& Thompson, 1998; Kollar \& Ailinger, 2002; Tabi \& Mukherjee, 2003).

“Americans [USA] have a curious attitude towards our own culture: we don’t think we have any. One of the great paradoxes of American culture is that the one constant Americans all agree on is that we are all different” (King \& O’Boyle, 2002, p. 1). For an American student travelling abroad, this trip is often their first experience and encounter with differentness when it is assumed that the whole world lives like “us” (Williams, Baumslag, \& Jeliffe, 1994). Culture is the “...prime mediator between ourselves and the world” (King \& O’Boyle, 2002, p. 2) and the “unveiling” of lived lives takes place when one is truly exposed and immersed in other cultures.

When one is the minority and everyday activities are carried out in a markedly differently fashion, differences become readily apparent. When an environment distinctly contrasts to "ours", when foods, furniture, housing, transportation, language, religious practices are unique to "our view”, the world and its many divergent features become readily apparent. Health care delivery is “foreign” or "strange”, with hospitals that don't look or function like “we” expect, and the nurses, midwives, and doctors have roles that differ from ours. There are new languages, expressions, new paralingual sounds and gestures. The physiologic processes are the same but there are myriad psycho/socio/cultural variations. Students are exposed to contrasting methodologies and for some the intimate contact with people of all colours and hues in all levels of society is a new experience. To whatever degree, partial or total, change brings about awareness of differences. Brueggemann (1987) stated “development comes through shatteringly in moves of harsh discontinuity...Indeed new development requires harsh displacement, a breaking of old configurations” (p. 36).This means that exposure and immersion in a developing world community will necessarily be more indelibly imprinted on one's cognition and affect and promote the greatest individual changes if old values are displaced/replaced. 
"In describing another culture, people tend to stress the differences and overlook the similarities” (Burnard, 2005, p. 177).Through immersion, students are "forced" to address that which is similar as they partake in family affairs/ceremonies that represent milestones. Food for survival, food for pleasure, we all eat. Jokes may not "match" one's expectations yet humour is extant in all societies. We all share bodily functions, the birthing process, national holidays, family structures and kinship systems even though each may be "played” out very differently. For students observing and participating in others' lives, all the sameness and differentness comes alive in the realisation of a shared humanity when in an international immersion programme. "My [author] work with people of many differing cultures, constantly reinforces my conviction that childbearing and child rearing are the foundations of the common human experience” (Levine, 1991, p. 143).

In an international immersion programme, of prime importance is active student involvement (Spradley \& McCurdy, 1972). Dubois (1963) wrote, "suitable supervision...a summer... of field experience may ultimately be far more important than all the data, problems, concepts, theories, methods or techniques that we impart in classes” (p. 36).

What is needed is true experiential learning. It is gained by practising nursing in another culture where affective and cognitive learning takes place through the direct interaction between client and patient. Cultural interchange and cultural growth are promoted as students work with their peers and colleagues in local hospitals and clinics, as students experience the life of others through the eyes of other, and as students fully realise their acceptance by their host communities and health care providers. It is the opening of hearts, minds, and eyes, the re-visioning; it is the acceptance; it is becoming "a family member" and a "professional colleague" that begins to alter the shape of one’s life. "Our lives are increasingly intertwined with those in other nations" (Warren, 2002, p. 300) as we share the very essence of who we are.

An interesting phenomenological study undertaken by Heuer and Bengiamin (2001) and Heuer, Russell, and Kahlstorf (1997) reflected on the international immersion of USA students in a short-term cross-cultural programme that took place 
in Russia. The writers (Heuer \& Bengamin, 2001; Heuer et al., 1997) focused on culture shock, defined as a clash between a known and unknown set of values as one is experiencing a "new culture". The honesty with which this article is written is commendable; perhaps other programmes have had similar experiences, yet, it has not been definitively reported as overwhelming. Students' impressions were clustered into three themes: airport customs, the physical environment, and public bathrooms. Students felt that they learned about differences in life styles yet their main thrust was focused on culture shock. Students felt that their cultural awareness had been piqued (aroused). “This...did not mean approval, but rather they recognized that the differences maybe were due to the structure and history of the culture” (Heuer and Bengamin, 2001, p. 8). Culture shock is a reality and the degree that each of us "endures" this phenomenon is dependent on the individual (Holstege, 2000; Inglis et al., 2000).

I can relate this phenomenon to one of the venues that I used. As we left the airport to travel the four hours to our destination, one student commented about the lovely rustic homes with their charming wooden shutters. Another student said "look at those old houses with the shutters hanging out of alignment and in need of paint”, each of the student's perceptions were unique and individualised. As individuals we have our own value systems that affect our perceptions of the world. For my students a point of consistent culture shock has been the return to the over-developed world and the everyday excesses.

Zorn (1996), using a descriptive survey design, found that the greatest changes in immersed students were: increasing international perspective (global understanding) and personal growth as individual respondents stated that trips enhanced self esteem, increased sensitivity to others, and helped one to become more open minded. Zorn stated a "lower impact was reported in [perceptions related to] the professional nurses' role” (Zorn) thus concluding that the research findings “support the long-range value of study abroad learning experiences” (p. 110).

St. Clair and McKenry (1999) posited that even short-term cultural immersion experiences helped individuals recognize their "unknown ethnocentrism" (p. 234). They found that students became "aware of how their ethnocentrism 
affected their ability to become culturally competent providers” (p. 234). Kollar and Ailinger (2002) found growth in practical knowledge, understanding, and personal growth. They further reported the influence of "interpersonal connections for nursing students [and] the direct effect on how, where, and why nurses chose to continue their professional practice after obtaining licensure.

Button et al. (2005) undertook a meta-analysis and integrative literature review on immersion programmes using a multitude of electronic databases. They reviewed the literature from $1980-2003$. Their findings included that “...preparation and debriefing were found to have [positively] affected the reported overall international placement experience” (p. 315) and that the “...primary effects of international placements were identified as personal development and transcultural adaptation" (p. 315). It was concluded that students should be "exposed to a variety of nursing experiences within the host country...as it gives them [students] a broad spectrum for comparison between cultures, nursing practice and health care delivery in those cultures” (p. 316).

\section{Summary}

This chapter has reviewed 45 immersion programmes that took place over a period of 21 years. The overall arching theme for initiating an international immersion programme was personal and professional growth. The programmes were analysed for purpose, characteristics of the programme, site, accompaniment by faculty, preparation for the student experience, and identified outcomes. There was almost $100 \%$ consensus related to the value and necessity of immersion programmes. I would like to conclude with this apropos quote:

We are affected in similar ways even though we may react differently. We react in ways that are affected by the way we perceive. We are affected by what we hear and by what we are told. We are moved according to how we interpret all of this and by what it means to us, whether it seems threatening or reassuring, disturbing, or quieting. (Salk, 1983, p. 94)

Chapter X, the concluding chapter, brings together the entire thesis. The evolution and importance of the study is discussed, as well as its limitations and the need for future studies about international immersion programmes. 
Table 9.1

International Immersion Programmes

\begin{tabular}{|c|c|c|}
\hline Year & Author & Title \\
\hline 2005 & $\begin{array}{l}\text { Button, Breen, } \\
\text { Tengnah, \& Baker }\end{array}$ & $\begin{array}{l}\text { The Impact of International Placements on Nurses' } \\
\text { Personal and Professional Lives: Literature } \\
\text { Review }\end{array}$ \\
\hline 2004 & Callister, L. & $\begin{array}{l}\text { Gaining a Global Perspective; The Impact of } \\
\text { International Undergraduate Clinical Electives. }\end{array}$ \\
\hline 2004 & $\begin{array}{l}\text { Evanson, T. \& } \\
\text { Zust, B. }\end{array}$ & $\begin{array}{l}\text { The Meaning of Participation in an International } \\
\text { Service Experience Among Baccalaureate Nursing } \\
\text { Students }\end{array}$ \\
\hline 2004 & Lee, $N$. & $\begin{array}{l}\text { The Impact of International Experience on Student } \\
\text { Nurses' personal and Professional Development }\end{array}$ \\
\hline 2004 & Leh, Robb \& Albin & $\begin{array}{l}\text { Responding to the challenge of developing a } \\
\text { global perspective in nursing education }\end{array}$ \\
\hline 2003 & $\begin{array}{l}\text { DeDee, L. \& Stewart, } \\
\text { S. }\end{array}$ & $\begin{array}{l}\text { The Effect of Student Participation in International } \\
\text { Study }\end{array}$ \\
\hline 2003 & Grant \& McKenna & $\begin{array}{l}\text { International clinical placements for undergraduate } \\
\text { students }\end{array}$ \\
\hline 2003 & Graves, $\mathrm{M}$. & $\begin{array}{l}\text { Lessons Learned from Valuable International } \\
\text { Experience }\end{array}$ \\
\hline 2003 & $\begin{array}{l}\text { Tabi, M., \& } \\
\text { Mukherjee, S. }\end{array}$ & $\begin{array}{l}\text { Nursing in a Global Community: A Study Abroad } \\
\text { Program }\end{array}$ \\
\hline 2003 & Walsh, L & $\begin{array}{l}\text { International Service Learning in Midwifery and } \\
\text { Nursing Education }\end{array}$ \\
\hline 2003 & $\begin{array}{l}\text { Walsh, L. \& De } \\
\text { Joseph, J. }\end{array}$ & I Saw it in a Different Light \\
\hline 2002 & $\begin{array}{l}\text { Kollar, S. \& Ailinger, } \\
\text { R. }\end{array}$ & $\begin{array}{l}\text { International Clinical Experiences. Long-Term } \\
\text { Impact on Students }\end{array}$ \\
\hline 2002 & $\begin{array}{l}\text { Watts, S., Law, K., } \\
\text { Ots, U., \& Waago, K. }\end{array}$ & $\begin{array}{l}\text { Reflection Across Boundaries: The European } \\
\text { Nursing Module }\end{array}$ \\
\hline 2001 & Anders, R. & A nursing study Abroad Opportunity \\
\hline 2001 & $\begin{array}{l}\text { Heuer, L. \& Bengamin, } \\
\text { M. }\end{array}$ & $\begin{array}{l}\text { American Nursing Students Experience Shock } \\
\text { During a Short-Term International Program }\end{array}$ \\
\hline 2000 & $\begin{array}{l}\text { Callister \& Hobbins- } \\
\text { Garbett }\end{array}$ & $\begin{array}{l}\text { "Enter to Learn, Go Forth to Serve": Service } \\
\text { Learning in Nursing Education }\end{array}$ \\
\hline 2000 & $\begin{array}{l}\text { Currier, C., Omar, M., } \\
\text { Talarczyk, G. \& } \\
\text { Guerrero, R. }\end{array}$ & $\begin{array}{l}\text { Developing and implementation of a Semester } \\
\text { Program in Mexico for Senior Nursing students }\end{array}$ \\
\hline 2000 & $\begin{array}{l}\text { Inglis, A., Rolls, C., \& } \\
\text { Kristy, Suzanne }\end{array}$ & $\begin{array}{l}\text { The Impact on Attitudes Towards Cultural } \\
\text { Difference of Participation in a Health Focused } \\
\text { Study Abroad Program }\end{array}$ \\
\hline
\end{tabular}




\begin{tabular}{|c|c|c|}
\hline 2000 & Holstege, M. & $\begin{array}{l}\text { The Lived Experience of Student Nurses } \\
\text { Providing Health Care Relief to Hurricane Mitch } \\
\text { Victims in Nicaragua }\end{array}$ \\
\hline 2000 & $\begin{array}{l}\text { Ryan, M., Twibell, R., } \\
\text { Brigham, C., \& } \\
\text { Bennett, P. }\end{array}$ & $\begin{array}{l}\text { Learning to Care for Clients in Their World, Not } \\
\text { Mine }\end{array}$ \\
\hline 2000 & $\begin{array}{l}\text { Scholes, J., \& Moore, } \\
\text { D. }\end{array}$ & $\begin{array}{l}\text { Clinical Exchange: One Model to Achieve } \\
\text { Culturally Sensitive Care }\end{array}$ \\
\hline 1999 & $\begin{array}{l}\text { Duffy, M., Harju, L., } \\
\text { Huittinen, L., \& } \\
\text { Trayner, C. }\end{array}$ & $\begin{array}{l}\text { An Innovative Model for International } \\
\text { Undergraduate Education }\end{array}$ \\
\hline 1999 & $\begin{array}{l}\text { St. Clair, A., \& } \\
\text { McKenry, L. }\end{array}$ & Preparing Culturally Competent Practitioners \\
\hline 1998 & $\begin{array}{l}\text { Colling, J., \& Wilson, } \\
\text { T. }\end{array}$ & $\begin{array}{l}\text { Short-Term Reciprocal International Academic } \\
\text { Exchange Program }\end{array}$ \\
\hline 1998 & Cummings, $\mathrm{H}$. & $\begin{array}{l}\text { Nursing in Barbados: A Fourth-Year Elective } \\
\text { Practice Experience for Nursing Students and } \\
\text { Registered Nurses }\end{array}$ \\
\hline 1998 & $\begin{array}{l}\text { Fennal, M., Fair, N., } \\
\text { Fleming, K., G Gibson, } \\
\text { S., Hugee, B., \& } \\
\text { Winborne-Tanner, M. }\end{array}$ & A Clinical Experience in Cultural Diversity \\
\hline 1998 & $\begin{array}{l}\text { Goldberg, L. \& } \\
\text { Brancato, V. }\end{array}$ & A United Kingdom Nursing Student Partnership \\
\hline 1998 & $\begin{array}{l}\text { Hadwiger, M., \& } \\
\text { Hadwiger, S. }\end{array}$ & $\begin{array}{l}\text { Transcultural Nursing Experience in the } \\
\text { Philippines }\end{array}$ \\
\hline 1998 & $\begin{array}{l}\text { Haloburdo \& } \\
\text { Thompson }\end{array}$ & $\begin{array}{l}\text { A Comparison of International Learning } \\
\text { Experiences for Baccalaureate Nursing Students: } \\
\text { Developed and Developing Countries }\end{array}$ \\
\hline 1998 & $\begin{array}{l}\text { Inglis, A., Rolls, C., \& } \\
\text { Kristy, S. }\end{array}$ & $\begin{array}{l}\text { The Impact of Participation in a Study Abroad } \\
\text { Programme on Students' Conceptual } \\
\text { Understanding of Community Health Nursing in a } \\
\text { Developing Country }\end{array}$ \\
\hline 1998 & Peel, K. & $\begin{array}{l}\text { Incorporating International Nursing in the } \\
\text { Curriculum: One Baccalaureate Program's } \\
\text { Experience }\end{array}$ \\
\hline 1998 & Purnell, L. & $\begin{array}{l}\text { University of Delaware Nursing Students Go to } \\
\text { Panama }\end{array}$ \\
\hline 1998 & Stevens, G. & Experience the Culture \\
\hline 1997 & $\begin{array}{l}\text { Heuer, L., Russell, L., } \\
\text { \& Kahlstorf, H. }\end{array}$ & Short-Term Cultural Immersion in Russia \\
\hline 1997 & Lee, $\mathrm{N}$. & Learning from Abroad: The benefits for Nursing \\
\hline 1997 & $\begin{array}{l}\text { Rolls, C., Inglis, A. \& } \\
\text { Kristy, S. }\end{array}$ & $\begin{array}{l}\text { Study Abroad Programs: Creating Awareness of } \\
\text { and Changing Attitudes to Nursing, Health and } \\
\text { Ways of Living in Other Cultures }\end{array}$ \\
\hline
\end{tabular}




\begin{tabular}{|c|l|l|}
\hline 1996 & $\begin{array}{l}\text { Ailinger, R. \& Carty, } \\
\text { R. }\end{array}$ & Teaching community Health Nursing in Nicaragua \\
\hline 1996 & Zorn, C. & $\begin{array}{l}\text { The Long-Term Impact on Nursing Students of } \\
\text { Participating in International Education }\end{array}$ \\
\hline 1996 & $\begin{array}{l}\text { Cedar Crest Hosts } \\
\text { Scandinavian }\end{array}$ & $\begin{array}{l}\text { Cedar Crest Hosts Scandinavian Nursing Student } \\
\text { Exchange Program }\end{array}$ \\
\hline 1995 & $\begin{array}{l}\text { Zorn, C., Ponick, D., \& } \\
\text { Peck, S. }\end{array}$ & $\begin{array}{l}\text { An Analysis of the Impact of Participation in an } \\
\text { International Study Program on the cognitive } \\
\text { Development of Senior Baccalaureate Nursing } \\
\text { Students }\end{array}$ \\
\hline 1994 & Bond, M. \& Jones, M. & Short-Term Cultural Immersion in Mexico \\
\hline 1993 & Bond, M., \& Jones, M & $\begin{array}{l}\text { Cultural Immersion: Student Experiences in } \\
\text { Mexico }\end{array}$ \\
\hline 1990 & Frisch, N. & $\begin{array}{l}\text { An International Nursing Student Exchange } \\
\text { Program: An Educational Experience that } \\
\text { Enhanced Student Cognitive Development }\end{array}$ \\
\hline 1986 & $\begin{array}{l}\text { Cotroneo, M., } \\
\text { Grunzweig, W., \& } \\
\text { Hollingsworth, A. }\end{array}$ & $\begin{array}{l}\text { All Real Living is Meeting: The Task of } \\
\text { International Education in a Nursing Curriculum }\end{array}$ \\
\hline 1984 & $\begin{array}{l}\text { Lindquist, G. } \\
\text { A Cross-Cultural Experience: Comparative Study } \\
\text { in Nursing and Health Care }\end{array}$ \\
\hline
\end{tabular}




\section{CHAPTER X}

\section{CONCLUSION: THE TIE THAT BINDS: PULLING IT ALL TOGETHER}

The most admirable thinkers within the scholarly community...do not split their work from their lives. They seem to take both too seriously to allow such dissociation, and they want to use each for the enrichment of the other... what this means is that you must learn to use your life experience in your intellectual work. Mills (1959, p. 195)

\section{Introduction}

Chapter X concludes with an overview of the entire project. It speaks further to who I am and how I have been shaped by my family of origin, personal development, and professional career. The significance of this study is discussed, as well as its limitations, and the important issue of the need for future research. I end with a conundrum for my readers as I have placed a vignette at the very end.

\section{The Evolution of This Project}

Growing up in a home that supported, provided, and treasured diversity in all its limitless shapes, forms and varieties moulded me into who I am and what I do (today). I have pursued a lifetime of exploring, learning, interacting, immersing, sharing, travelling, and, especially caring for others. I internalised the socialisation process that I lived as a young child, adolescent, and young adult. My roots were planted and nurtured by my extended family and sprouted, grew, and took hold as I matured. The many branches have enabled me to glide easily among countries, continents, languages, and most of all people. Nursing was a natural profession to pursue, as caring has always been the priority of my life. The most important "piece" of my chosen profession is the psychosocial interactions and trust that emerge between patient/family and me as a caregiver. 
There is something about nursing, about intimacies that are formed, within minutes or hours, in circumstances that require complete commitment by all involved. Nursing is the catalyst that allows me to pursue that which I cherish and value, people and relationships, that which represent the very essence of my being and my profession.

As I initially practised my profession and gained expertise in more than one clinical speciality in the USA and abroad, I began to feel the need to delve deeper into the issues of human connection, status, and roles and how all these factors affect a lifetime of living.

I did not want to become a clinical specialist but rather to pursue studies that transcended the twigs and branches of nursing and penetrated the deeply enmeshed roots. Sociology and anthropology were the way to go, and I found a graduate programme in Transcultural Health at Pennsylvania State University (USA) that focused on the health care concerns that have always been critical and central to my life and profession. The subject areas were "bundled" and intertwined, as the focus was people, health, and the bio/psycho/socio/cultural components of life that impact the individual, family, and a community's health status throughout the life span.

Many years ago I learned that the basis of the profession of nursing was the nurse-patient relationship, which I constructed as a partnership. In my years of nursing practice, I saw another reality, exacerbated by perceived “differences” of patient and care provider (fit) and the power shifts contained therein.

As a graduate student, I taught undergraduate nursing classes but did not anticipate becoming an instructor. What struck me was the neediness and needs of students to discuss and express overwhelming feelings about "routine” patient care. They had need to be in a safe environment in order "to talk". They had the need to be nurtured in order to "grow" and address the perceptions that could lead to "new" insights and awareness. They had to be supported as they gained an expanded world view. I learned that personal change can have a profound effect on nursing practice.

When I began teaching at Humboldt State University, I realised the "vastness of the gap" between students and the "rest of the world". Students were focused on their lives and getting through the nursing programme and had little or no 
knowledge of how others lived. Perceptions were formed in the context of their socioeconomic status, ethnicity, race, values, and their individual socialisation process. Students repeatedly conceptualised the world from their own middle class life style(s). I felt impelled to develop an international programme with a maternal child health focus in order to both teach and breach a perceived lack of diversity. Students became sieves, absorbing with all their senses their "reality" as they experienced international immersion, and students were fundamentally changed. Benhabib (1992) succinctly stated that one becomes more aware of who one is by communicating and connecting with those who are not like us.

What has emerged from the immersion programmes are astounding metamorphoses in students, cum nurses. As their world view grew and changed, both professionally and personally, immersed students assumed an expanded role and responsibility for their psycho/social/cultural growth. This was exemplified by recognising the humanity in all of us via identifying our human similarities. Immersed students also recognised common human needs and desires and how individuals and groups problem solved to reach their goals. Students looked anew at themselves, as persons of privilege, due solely to the vagaries of their geographical birth. Students addressed caring and the importance of the individual and her/his family, as well as patient advocacy as absolute necessities. They realised that injustice and intolerance are alive and well both in the venue countries as well as their country of origin. Students addressed change and how it begins with oneself. Finally, students came face to face with blatant prejudice that engendered deep reflection and the realisation that in the USA racism and stereotyping need to be addressed, issues that students really didn’t think about before.

Campesino (2002) addressed the same concepts when discussing nurses making comparisons between health care systems in developing nations and those of developed nations: "The abuse of power in such comparisons and the reluctance of nurses from the United States to address conditions of suffering in their own country” (Campesino, 2002, p. 343) must be attended to. Also, "new insights and solutions for our own problems might be found from comparisons of health care systems outside the United States if we ascertain what is working well” (Campesino, 
2002, p. 344). The dialectic should be addressed in all facets of nursing as well as in immersion programmes as students (and nurses) come face to face with opposites and conflicting ideas and the absence of social justice (Redman \& Clark, 2002).

Immersed students also discussed their professional role changes. Role change was described as: proactive advocacy for patients and families; confidence, ability, comfort, enjoyment and basking in anticipated challenge when working with individuals and families with different value systems; constant awareness of the health care institution's power as well as cumulative effects on clients of the totality of all the sociologic institutions and how all come together as a patient (and family) seek health care, and the potential preconceived "thinking” and stereotyping by health care providers about those that "differ". The new dimension to role change came about through expansion of world view via exposure, immersion, willingness to take risks, increased flexibility and adaptability, increased maturity, increased calmness, willingness to "stand up" for clients and self in professional encounters, comfort with being assertive to promote change, and challenge the status quo.

Participants talked about their consciousness and attentiveness to the utilisation of supplies and felt that their homebound colleagues just did not understand the issue of needless waste directly related to overall health care costs. Colleagues were not aware of alternatives.

For me the greatest change, through the immersion programmes has been in my openness in "teaching from the heart”. As I watch the profession of nursing become increasingly technological (Leenerts, 2003), I envision robots moving from bed to bed "passing meds". I literally cringe. The art of nursing is of supreme importance; nurses must address care and caring by looking at patients in their [patients] own "life environment". "The art of nursing is not an indulgent nicety, but instead, an essential activity grounded by practice and manifest in helping patients create coherence and meaning in [their] lives” (LeVasseur, 1999, p. 62). The cumulative effects of each patient's individuality must be valued in order to develop meaningful relationships that promote a healthy lifestyle. (P. Stevens, 1989) “The identity accepted by the individual...[is] the most important” (Barer-Stein, 1994, p. 156) marker in the nurse/patient relationship. Communication must become and 
remain among the top priorities of the profession, and that is what I have relied on in my teaching. For example at post clinical conferences following clinical rotations, and in students' written work, I encourage students' reflections and analyses to be an integral component. I realise how institutional racism, policies and procedures negatively impact people by affecting the quality and quantity of care offered. For example, even in the USA, at change of shift report, judgements about patients are often transmitted to the oncoming shift. Comments are made about patients to the students during the workday, and the attitude of the nurses and the effects, when working with certain patients is judgemental and demeaning. I teach to recognise racism, stereotyping, and the negativity that ensues and pervades the atmosphere of patient/family care. I focus on giving unconditional client centred care. In my evolution as a professional person I realise that although the "culture" of a particular society affects health care, it is the "culture" of the five sociologic institutions (economics, education, family, government, and religion) that have the greatest cumulative impact on individuals, families, and community life. In my perception, nursing has focused on separation; emphasising differences among people rather than similarities. We must address patients through their perceived culture and honour each for who he/she is. The nurse that may be "expert” on a particular culture can still have deeply held racist beliefs. We need to go beyond culture to practise nursing in the USA and the world addressing human dignity and human worth.

The decision to undertake a PhD at the age of 62 and successfully complete it at the age of 67 was also part of my evolutionary being. My thesis was necessitated by the paucity of literature about the profound effects of international immersion programmes upon nursing students. This thesis was and is, necessary, in order for me to disseminate my life's work. The programmes were transformative to both my students and myself in opening an unknown, unfathomable place for all of us.

My thesis is a culmination of how I see myself and who I am. It is the coming together of my heart, soul, and spirit as I endeavour to inculcate in students the importance of acceptance and understanding of differences, the commonality of 
humanness, creativity, innovativeness, and "taking risks". It is always conducted by my being there for students to explore and express their feelings in a safe place, about everyday happenings in the developing world.

Students and I usually develop long term relationships. I support them through projects undertaken long after their trips, often to do with the giving of self, the advocacy to stop intolerance in its tracks, the ability to support patients to the fullest, and the understanding and comfort to champion diversity.

\section{Why This Study Matters}

Simply said, immersion programmes change students. Participants initially come from a "different place" in their abilities to understand and work well with people whose values greatly differ from their own. People whose colour is different are gradually viewed in a "new light" as students realise the daily hurdles faced by such individuals solely due to their different looks. Former participants are challenged to "educate" their professional peers, neighbours and friends. Their world view is broadened as students mature. Finally and metaphysically participants look forward to and are excited to work with widely different others. They have an openness that continually broadens their overall world view.

The process, however, is slow. In the countries where we work, we break down barriers together. The "locals and we", as we immerse ourselves in the lifestyles and health care systems, become part of the social rubric of that community. The interaction among all is one of learning, sharing and giving of oneself. For USA students the realisation that the rest of the world does not live like us is especially powerful.

To the profession of nursing, the emphasis is on health and well being no matter who you are and where you live. The tools that the students return with enhance their skills in all personal and professional realms. It is especially relevant when students are care providers of patients and families who perceive their care to be biased because of "who they are". For this reason programmes are constructed so that the venue changes with each programme so that I may be a role model and work 
with my students in assessing communities, health care systems, health care delivery, patient care, family care, and patient care.

An incident I vividly remember is a day that we spent in a leper colony. I could hear the comments among my students "It must be okay to touch people, look at MaryAnne”. This quickly facilitated robust introductions, trust, and a comfort level that might not have been possible had not the students had a role model. There might have been awkward feelings and restricted interactions given the environment and the fear generated by a diagnosis of leprosy. By being there as a support system for students I was able to eliminate students feeling ill at ease, fearful or offended. By role modelling appropriate caring behaviours I was able to advocate and facilitate an interactive learning experience.

Students quickly gained confidence in their role and functions as their comfort level increased. By modelling each step the student transition was eased, and made for independent action of participants. As the professor, I work with my students at all the clinical sites, share living quarters, and participate in the activities of daily living.

There is purpose and meaning to what I do by immersing students in the lives of others. This opens a "brave new world”, sans technology, emphasising instead the therapeutic use of self. The art of nursing is resurrected in its primacy. A dimension renewed, "nursing is a caring profession...Caring encompasses empathy for and connection with people...Teaching and role-modeling caring is...nursing” (Fahrenwald, Bassett, Tschetter, Carson, White, \& Winterboer, 2005, p. 46). To me, being entwined in students' professional and social evolving world view is embedded in the role of teaching and also has very personal meaning. It is as if a bud, on its tentative journey to maturity, needing nurturing in the forms of water, sun, and soil, begins to blossom and the flower is the culmination.

International immersion surpasses cultural sensitivity, awareness, and competence. Immersion surpasses nursing as these journeys allow people in all circumstances to experience and share humanness. It is the grass roots of positive change for a world that "preaches" globalisation. It is a world of exposure, a window to all of humanity and life styles because: 
The existence of global means of cultural dissemination adds an entirely new element to multiculturalism (and to multilingualism). It ensures that pluriculturalism is here to stay, that it will intensify, and that it is linked into all aspects of public life: economic, political, social and all aspects of the private. (Kress, 1995, p. 19)

It is also learning that the majority of the world's people live in dire poverty, and have no "exit", nor a safety net of help. It is being able to begin to make professional and personal changes in one’s life based on social justice.

\section{Study Limitations}

This study is the culmination of many years of professorial and experiential endeavours which have been scrutinised and analysed to become my doctoral thesis. Students who participated in the immersion programmes were asked to become part of this project by consenting to be interviewed. The aim was to further understand the outcome of each student's experiences at the time of immersion by looking for patterns and themes across the participant range and if, and how, the programmes impacted on their lives (Nieswiadomy, 2002). Appropriateness of the sample population was met because the participants could “...best supply [the] information according to the conceptual requirements of the study” (Polit et al. 2001, p. 250) and yielded “...the fullest possible understanding of the phenomenon of interest (p. 250).

The sample was one of convenience, because of easy accessibility and financial considerations, but did not give all participants equal access randomly and thus can be biased. However, "the phenomenon of interest is [was] homogenous with respect to the characteristics studied" (Gillis \& Jackson, 2002, p. 506) and is thus acceptable as a research methodology. As I used a convenience sample it is not possible to extrapolate to a larger population. Former participants, who lived in geographical proximity to me, were contacted. I originally planned to interview six former participants but received permission to increase the number to ten, as I felt that a greater sampling would yield more comprehensive results.

I sent out letters of invitation to participate and each person contacted volunteered to be included in the study. The volunteers may have had their own reasons, agenda, for their willingness to participate, which has the potential to skew 
the results. The interviewees were well known to me and this factor can also bias the results because participants might have espoused what they presumed I wanted to hear. The positive side of knowing the participants, is that each can truly feel comfortable expressing what he/she feels, can be more open, more disclosing, and more willing to discuss their lived experiences. "Qualitative research focuses on gaining insight and understanding about an individual's perception of events” (Nieswiadomy, 2002, p. 51) yet even when getting rich thick information it may be irrelevant, and it may be one's own agenda.

Three to thirteen years had elapsed, depending on the year of participation, since the immersion experience and this might have caused some lapse of memory for participants. Memories may not have been as crystal clear, yet many memories, especially those deeply felt, engrained, and powerful were in evidence. Maturation, the gap between the end of the experience and the present could have affected the results as a result of the passage of time (Polit et al. 2001).

Self report “...the most common method of data collection in nursing studies-are strong in respect to their directness” (Polit et al. 2001, p. 277) in ascertaining what individuals “...feel or what they believe” (p. 277). The weakness can be the level of openness and truthfulness of the participant in their self report (Polit et al.).

Initially, for each programme there were 40 students who wished to participate, but due to constraints at the site, each programme could accommodate a limited number, which varied. Participants were chosen by random selection, and the possibility exists that outcomes may have been different depending upon individual students and the bonding and attachment processes that ensued. Groups that form at random, individual personalities and how each mesh with another, added to environmental circumstances, and leadership can yield multiple differing outcomes. If individuals have personal conflicts the group process can be deflected but if the group works together in concert, the unity allows exploration of feelings on an individual and group basis. The environment (physiologic, psychosocial) also contributes to the potential of a positive and/or negative outcome. The leadership of the group is very important in contributing to positive outcomes and directly relates 
to the use of therapeutic communication and psychosocial support at both the individual and group levels.

The trips were self-financed, myself included. Students of all income levels were participants. Some used students loans, others borrowed money from family and friends, and some students used their own savings. For one programme there was a fund-raiser; all the proceeds were divided equally among students and used toward their immersion programme expenses. For three of the programmes, I sought grants from Associated Students of Humboldt State University and students, again, received equally divided monies to use toward their airfares.

Also, a bias might have existed as to the “makeup” of students' who were willing to take risks, and were adventuresome versus students who would not choose an option to travel and put themselves "at risk". I must acknowledge the difficulty in making immersion programmes available to every student. The experience of international immersion is a limited opportunity at present; in my programme it is due to the availability of accommodations, and clinical placements at the site. The process that I use is random selection from all interested students. For universities, international immersion programmes can mean a huge expenditure of monies in supporting students in these endeavours. The value however is that international immersion for all students is the key to the viable future of this world because in order to break down barriers one needs to honestly experience the world of another. Dilthey (as cited in Habermas, 1968/trans. 1971, p. 156) stated:

An infinite wealth of life unfolds in the individual existence of particular persons in virtue of their relations to their milieu, to other persons, and to things. But every single individual is at the same time a point of intersection for structures that permeate individuals, exist through them, but extend beyond their lives.

\section{Future Studies Needed}

A vision is only an idea or an image of a more desirable future, but the right vision is an idea so energizing that it in effect jump starts the future by calling for the skills, talents, and resources to make it happen. (Nanus, 1992, p. 8) 
Because of the power of the findings of this thesis, immersion experiences are clearly important to nursing students’ learning. Faculty who undertake immersion programmes need to be experienced and comfortable in working in international settings and need to be comfortable in living situations that greatly differ from their usual lifestyle. Without faculty and lack of support systems, yes, students have each other, but they lack the co-ordination and leadership that can take the immersion experience to the very depths and core of living (life).

I utilise Diekelmann's (1993, 2001) theory of narrative pedagogy as we explore everyday experiences and the effects on students, potential effects on patients, families, communities, and societies. The settings for these fora set a tone of openness, dialogue, expression of feelings, learning, coping, and support. There is continuing support by the professor as well as the students’ peer group. As a profession we must recognise and begin to combat the overwhelming effects of racism, particularly institutional racism, in all its forms. Learning about cultures, and other value systems, is certainly a valid premise, but how will change occur on the political level if we as nurses do not question the status quo. "Critical reflection leads to knowledge” (Jacobs, Fontana, Kehoe, Matarese, \& Chinn, 2005, p. 9) and emancipation, and “improves the human condition” (Jacobs et al., p. 13) by learning about dialectics and making life changes. This study identifies similar themes as does prior research and adds the dimension of long term outcomes, only focused upon in a handful of studies. The dialectic, as found in this study, has not been addressed before, and herein lays the need for further research. All nursing students need to be exposed to themselves above all, and be able to critically reflect upon who they are and how they function within their professional and social environments. Dialogue, support, and expression of feelings must be addressed as students continually are exposed to lifeways as lived by their patients, and others. By continually increasing one's knowledge about humanity, both its similarities and differences, in the affective and cognitive realms, transformation is possible: "A World Shrinking, a World Expanding”. Devereau (1967) succinctly paraphrased the great scientist Poincaré (1901/trans. 1926) when he stated the “definition of science 
as a quest for similarities in differences and for differences in similarities” (Devereau, 1967, p. 255) which aptly applies to the art and science of nursing.

Future nursing research must address social justice if the profession is to continue to evolve in its service to global humankind. In order to recognise and understand social (in)justice and all its ramifications, students need to be exposed internationally and in their home countries to the existent dichotomies, the political process, and the making of health policy. There is a definitive need for social justice to be taught in nursing programmes in all nations because nursing does not operationialise itself in a social vacuum. (Callister \& Hobbins-Garbett, 2000; Fahrenwald et al., 2005; Georges, 2003; Redman \& Clark, 2002).

Future research should extend the findings of this study to better understand ourselves, to better understand cultures, to make a difference in the world, to promote nursing as a profession that champions social justice, and to promote further understanding of nursing education in relationship to culturally appropriate nursing care.

\section{Summary}

This chapter has pulled all the "pieces" together by discussing the evolution of the project, why this study matters, its limitations, and the need for further research. On a final note, I am providing a vignette, upon which I will not comment, but encourage your interpretation.

The Boy on the Train

My seven year old [older daughter] and I are travelling from one big city to another on a train that will take us about thirty-six hours until we get to our destination. There are bars on the windows; we sit on hard wooden seats, six people to a pallet. It is appallingly hot and humid. We have brought along all our provisions and drinks to help avoid dysentery once again. We have been travelling about three hours and an employee comes along closes the wooden shutters on all the windows, and locks the entry/exit doors in each railroad car. The train slows as we go through a mountain pass. An English speaking passenger explains that we are going though a mountain pass known for thieves who jump upon the tops of the railway cars, and descend 
and rob all the passengers, many times maiming and killing. I feel as if we are partaking in an epic American western; only this is not fantasy but fact (reality). There is a little boy about eight years of age who got on the train about an hour ago. He is slowly wending his way through the railroad cars with a little hand brush made of locally picked leaves. He brushes off shoes, brushes away discarded fruit, drinks, and other bits of left over food. Some people give him a small monetary token of appreciation, others push or even kick him away. When he has brushed all the debris into a corner of the railway car he alights, crosses the track and waits for a train going in the opposite direction. Sometimes he may get thrown off the train because he does not have a ticket, at other times the conductor will pretend that he is not there. There are times when he must give the conductor a percentage of his earnings. This eight year old is helping to support his family. 


\section{CODA}

“...a place where temperature and color and feeling were somehow more elemental, more penetrating, capable perhaps (who could say for certain?) of reaching through the organs of sense to leave their direct and permanent impress on the soul” (Zencey, 1995, p. 4). 
APPENDIX A

INVITATION TO PARTICIPATE 


\author{
Invitation to Participate \\ Transforming Experiences: \\ Facilitating Student Nurse Development through Immersion in \\ International Programmes
}

Researcher: MaryAnne Levine

I am a PhD student at the above university in the Graduate School of Nursing and Midwifery. I am undertaking a study of the outcomes, both personal and professional, of international immersion programmes in the developing world for undergraduate student nurses.

I am inviting six former students who have participated in one of the International Transcultural Perinatal Programmes to participate in this study. Participants will be asked to partake in a semi structured hour long taped interview. Inclusion in the study will be based on current geographic proximity to the researcher.

The information that follows describes what will take place if you decide to participate.

\title{
INFORMATION FOR PARTICIPANTS
}

1. Each participant will meet with the researcher for one hour or more at a place of the participant's choosing or when necessary the interview will take place on the telephone.

2. The interviews will be semi structured with broad questions such as: Please reflect on what it was like for you going to work and live in a country in the developing world? Tell me the story of your experience. What impact did the experience have on you personally? What impact has the experience had on your professional practice. The interview will be audio taped.

3. Each participant will choose a pseudonym to be used throughout the entire research process.

4. Confidentiality will be maintained at all times. All records will be kept under lock and key and only the researcher will know the true identity of the participants.

5. The taped interviews will be transcribed by a professional typist and the participants will only be known by their pseudonyms. Data will be kept in a locked filing cabinet and only the researcher will have the key.

6. Participants will be asked to read the transcripts of their taped interview for verification and accuracy. Participants may alter or correct the transcript to clarify comments they have made. This may be done up to the commencement of data analysis. Each participant will be provided with a copy of her/his transcript of the taped interview. 


\section{VICTORIA UNIVERSITY OF WELLINGTON \\ Te Whare Wananga o te Upoko o te Ika a Maui}

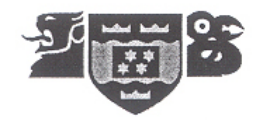

7. The tapes will be returned to the participants at the end of the study.

8. Each participant will not know the identity of the other participants.

9. The results of this research will be in the public domain. It may be used for professional presentations and/or in published articles.

10. The transcripts will be kept for six years after the conclusion of the research project for possible use in further articles, professional presentations, or further data analysis.

If you are interested in participating in the research, please contact me by telephone or email me at the following address: levinem@humboldt.edu, or telephone 826-5137(W), 826-1322(H).

I will arrange for you to be sent a consent form to sign and we will organise a mutually agreeable time and place for the interview to occur.

Ethical approval has been granted for this research from the Human Ethics Committee of Victoria University of Wellington. If you would like to discuss any ethical issues concerning the project with them, their contact telephone number and email address are as follows:

allison.kirkman@vuw.ac.nz or telephone 64-4-463-5676

If you wish to discuss any other aspect of the project you may contact my $\mathrm{PhD}$ supervisor, Professor Maralyn Foureur of the Graduate School of Nursing and Midwifery at Victoria University of Wellington by telephoning 64- 4- 4636137 or email maralyn.Foureur@vuw.ac.nz.

Thank you for your interest in this study. I look forward to hearing from you.

Yours Sincerely,

MaryAnne Levine 
APPENDIX B

CONSENT FORM-TRANSCRIBER 


\section{VICTORIA UNIVERSITY OF WELLINGTON \\ Te Whare Wānanga o te Ūpoko o te Ika a Mãui}

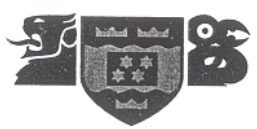

\section{Confidentiality Agreement for Transcriber of Audio-tapes}

I, Brenda Watson, agree to maintain the confidentiality of the participants in this research project:

TRANSFORMING EXPERIENCES:

FACILITATING STUDENT NURSE DEVELOPMENT THROUGH IMMERSION PROGRAMMES in INTERNATIONAL SETTINGS,

by keeping the contents of the audio-tapes and the typed transcripts confidential.

I will return the audio-tapes and the transcripts to MaryAnne Levine when completed.

I will not make copies of the audio-tapes or the transcripts for the use of anyone other than the researcher MaryAnne Levine.

Signature of Transcriber:

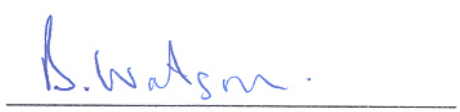

Date:

1 July 2003 
APPENDIX C

CONSENT FORM-PARTICIPANT 


\section{VICTORIA UNIVERSITY OF WELLINGTON \\ Te.Whare Wananga o te Upoko o te Ika a Maui}

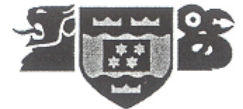 \\ CONSENT FORM}

Consent to Participate in a $\mathrm{PhD}$ study to be conducted by MaryAnne Levine entitled: Transforming Experiences:

Facilitating Student Nurse Development through Immersion in International Programmes

I have been given, and understand, the explanation of this research project. I have had an opportunity to ask questions and have them answered to my satisfaction.

I consent to be a participant in this study that addresses transcultural immersion programmes in the developing world for baccalaureate undergraduate nursing students.

I have completely read, and understand, the accompanying "Information for Participants".

I am fully informed that I may drop out of the study at any time of my choosing.

I understand that I will be given a copy of the transcript of my interview to comment on and correct if necessary.

I understand that I may amend my interview transcript up until the start of data analysis.

I understand that I will be given the audiotape of my interview at the end of the research project.

I understand that I will not be identified in any publication arising from the research.

I understand that I will only be known by the pseudonym that I have chosen.

The researcher is the only person who will know my true identity and that will remain confidential. All data will be kept in a locked cabinet and only the researcher will have the key.

Signature of Participant

Date

GRADUATE SCHOOL OF NURSING AND MIDWIFERY

PO Box 600, Wellington, New Zealand, Telephone $+644463-5363$ or Freephone 0800 108-005

Facsimile+644463-5442, Email Nursing-Midwifery@vuw.ac.nz 
APPENDIX D

PREAMBLE UPON TAPING 


\section{VICTORIA UNIVERSITY OF WELLINGTON \\ Te Whare Wananga o te Upoko o te Ika a Maui}

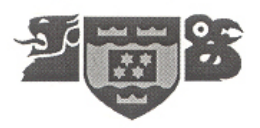

\section{PREAMBLE UPON TAPING}

Just to remind you that this is being audiotaped and also to let you know again that this data will be held in the strictest confidence and kept under lock and key.

I am the only person who will know your true identity as we will be using your pseudonym for taping and your demographic data is also under a pseudonym.

I have a 3 by 5 card here with your pseudonym so that I have a constant reminder to call you by that name.

This tape will be returned to you at the completion of my PhD.

This interview is purely voluntary and there is no financial remuneration involved. You are free to terminate the interview at any point, for any reason.

The taped interviews will be transcribed by a professional typist in New Zealand who will only know you by your pseudonym.

You will be asked to read the transcript of your taped interview for accuracy and further clarification can be offered at that time.

I may just take a few notes so that I can make sure that I am listening carefully as I don't want to interrupt you or miss anything.

Do you have any questions at this time?

I'm going to give you the demographic data form that you completed so that you can reiterate the information for this recording. 
APPENDIX E

ETHICS APPROVAL 


\section{VICTORIA UNIVERSITY OF WELLINGTON \\ Te Whare Wananga o te Upoko o te Ika a Maui}

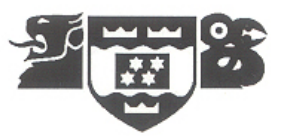

\section{E M O R A N D M}

DATE: June 17, 2003

TO: $\quad$ Mary Anne Levine

FROM: $\quad$ Allison Kirkman, Convener, Human Ethics Committee

SUBJECT: APPLICATION FOR ETHICAL APPROVAL:

Thank you for your application for ethical approval, which has now been considered by the Standing Committee of the Human Ethics Committee.

Your application has been approved and this approval extends until December 2005.

Best wishes with your research.

Allison Kirkman

Convener, Human Ethics Committee 
APPENDIX F

EXAMPLE OF TRANSCRIPT PAGES WITH ANALYSIS 
the world. They way they treated us and brought us into their wist just because we were nurses and from homes and shared every another country and they wanted to

Can you tell me more about that? The camaraderie you're talking about of nurses?

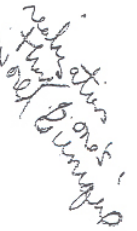

Well I think that here you know in this little community when we meet another nurse, oh you're a nurse and then all of a sudden there's this understanding ah, you know. Where do you work what do you do. We thing about them and what they're going through and when you know something aboutd and you meet somebody's who's a nurse then you do start a foundation of a have an understanding and somewhere relationship. Of course, everywhere is diferent and with us. I would like great in terms of the nurses and everythin

to be able to do more to thank them for it.

It sounds as if you have reflected so much about this experience, from your point of view have the nurses ever talked to you about how they felt about having been working with you?

wanted to be there and that we were working with them. They were glad that we had traveled to Nakhodka. They forgotten by the world and forgotten by their own country and felt sort of fout how it seemed like Moscow had forgotten about Nakhodka, they talked about how it seemed llke Russia. For them to have a group of Ams. They were very flad and excited they had to share with us was a surprise hospital with us. They were very $D_{i}$ about it and didn't want to just share the hospitar our institutions would share

open and showed us things that I'm not sure that our institutions wro

with a foreigner.

Can you tell me some more about the sharing, you're not sure that they would share?

Our own positions?

Yeah

Well I think that when we share our institutions and we have a foreign person come to visit that we show them the pretty, pretty things and guide them theugh with a bunch people to show them the things that they want them to 作 see. When wussia, which is not always pretty and often hard.

Not always pretty and very hard.

Yes. They shared that with us. They knew what we were coming from Yes. They shared that with us. They knew what we were coming
because they'd seen western hospitals on TV and in movies. They knew we
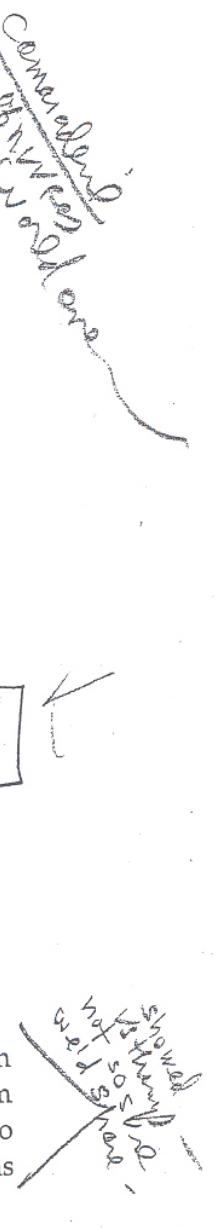
had many things like medications and equipment that they don't have. In addition to sharing the hospital with us and the care and the-things that they don't have, they shared their lives with us. They wanted us to be involved, to go places with them, and to meet their families and the people that mattered to them.

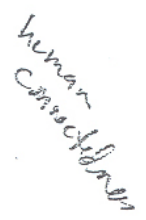

So it sounds as if you were immersing in the healthcare system and immersion in the social system?

Yes.

And the instant identification and trust because you were a nurse and they were a nurse?

Yes, and the fact that we had come to learn from them too. That we weren't coming there to show them what American nurses do.

I hear. what you say and tell me some more about what you or we were going to learn from them.

Well they wanted to share with us everything that they do in addition to labor and delivery, to the pediatric hospital, the care of the baby. I think back to the medical hospital where they shared so much with us that wasn't pretty. They showed us things like their ICU where they don't have things like our IV fiuids or IV pumps. Every time the patient needed IV fluids we had to get a new needle to put in their vein, the IV fluid ran in by gravity, and then when it was done the needle came out. (There's just so much that we have.

Addendum:

I wanted to learn about who the Russian people and healthcare workers were as people and how their culture influenced the care that they give as nurses to the patients. In addition, how the culture of Russia influenced the way that people approached"their health care. I learned that the health care workers in Russia are incredible dedicated people full of compassion. They putt up with circumstances that we would find intolerable because they îne their community and caring for people. The Russian people are used to the poor circumstances in their health-eare system. They do whatever it take to help their loved ones in the hospital, such as bringing in sheets, food and medicine for their loved one that the hospital can't provide.

What overall meaning did the trip have for you? Can you reflect on that?

I think that the biggest, oh gosh there's so much about that trip, it's hard to put it all into words because it's like it went somewhere in my heart and that was that. In addition, I think about the fact that people and everything that they were willing to share with my co-workers my colleagues, me. When we
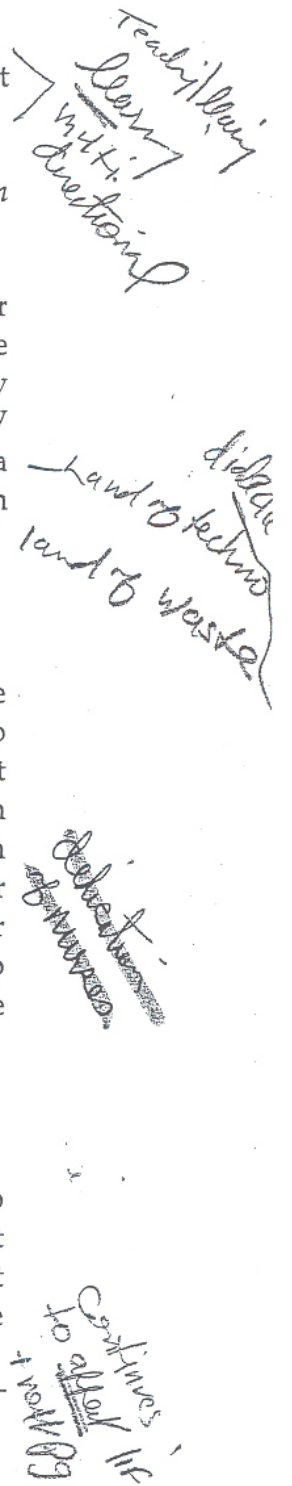


\section{REFERENCES}

Accord, L. (2000). Education: It's about students. Journal of Professional Nursing, 16(3), 128.

Ackerly, B. (2000). Political theory and feminist social criticism. Cambridge: Cambridge University Press.

Agarwal, B. (1994). Gender and command over property: A critical gap in economic analysis and policy in South Asia. World Development, 22(10), 1455-1478.

Ahmed, L. (1988). Between two worlds: The formation of a turn-of-the-century Egyptian feminist. In B. Brodzki, \& Schenck, C. (Ed.), Life/lines (pp. 154174). Ithaca, New York: Cornell University Press.

Ahmed, S., Adams, A., Chowdhury, M., \& Bhiuya, A. (2000). Gender, socioeconomic development and health-seeking behaviour in Bangladesh. Social Science \& Medicine, 51(3), 361-371.

Ailinger, R., \& Carty, R. (1996). Teaching community health nursing in Nicaragua. Nursing and Health Care, 17(5), 236-241.

Alibhai, Y. (1986). Can't they see I'm me. Nursing Times, 82, 56.

Allport, G. (1942). The use of personal documents in psychological science. New York: Social Science Research Council.

Ambrose, S., \& Bridges, M. (2005). The necessity of really knowing your audience. National Education Association, 22(3), 6-7.

Anand, S., \& Bärnighausen, T. (2004). Human resources and health outcomes: Cross-country econometric study. The Lancet, 364(9445), 1603-1609.

Anders, R. (2001). A nursing study abroad opportunity. Nursing and Health Care Perspectives, 22, 116-121.

Anderson, B. (2005). Assessment of the World Bank's support for reproductive health. [Review of the book Banking on Reproductive Health]. Journal of Midwifery and Women’s Health, 50(4), 352.

Anderson, R. (1989). Kierkegaard on ethics in communication with self. In C. Roberts, K. Watson \& L. Barker (Eds.), Interpersonal communication processes (pp. 411-440). New Orleans: SPECTRA. 
Auden, W. H. (1967). A short defense of poetry: Tradition and innovation in contemporary literature. Paper presented at the International PEN Conference, Budapest.

Awareness mobilization. (2000). Awareness, mobilization, and action for safe motherhood: A field guide. Washington, D. C.: The White Ribbon Alliance for Safe Motherhood.

Aye, M., Champagne, F., \& Contandriopoulos, D. (2002). Economic role of solidarity and social capital in accessing modern health care services in the Ivory Coast. Social Science \& Medicine (In press still as of Oct 02).

Barer-Stein, T. (1994). Culture in the classroom. In T. Barer-Stein \& J. Draper (Eds.), The craft of teaching adults (pp. 145-164). Florida: Krieger Publishing Company.

Barer-Stein, T. \& Draper, J. (Eds.). (1994). The craft of teaching adults. Florida: Kreiger.

Barlow, M. (2005). Abolishing "effortless perfection". National Education Association, 23(2), 6-7.

Barnard, D., Towers, A., Boston, P., \& Lambrinidou, Y. (2000). Crossing over: Narratives of palliative care. New York: Oxford University Press.

Barnes, P. (1981). Education through autobiography. Milton Keynes: The Open University Press.

Barnhart, C. (1969). The American College Dictionary. New York: Random House. Barrera, A. (1990). The role of maternal schooling and its interaction with public health programs in child health production. Journal of Development Economics, 32, 69-91.

Behar, R. (1996). The vulnerable observer: Anthropology that breaks your heart. Berkeley: University of California Press.

Belenky, M., Clinchy, B., Goldberger, N., \& Tarule, J. (1986). Women's ways of knowing. New York: Basic Books.

Beneria, L., \& Bisnath, S. (1997). Gender and poverty: An analysis for action. New York: United Nations Development Program. 
Benhabib, S. (1992). Situating the self: Gender, community and postmodernism. New York: Routledge.

Benner, P. (1984). From novice to expert. Menlo Park: Addison-Wesley Publishing company.

Benner, P. (1999). Claiming the wisdom and worth of clinical practice. Nursing and Health Care Perspectives, 20(6), 312-319.

Benner, P. (2000). The roles of embodiment, emotion and lifeworld for rationality and agency in nursing practice. Nursing Philosophy, 1, 1-14.

Berg, B. (2001). Qualitative research methods for the sciences (4th ed.). Boston: Allyn and Bacon.

Bertaux, D. (1981). Introduction. In D. Bertaux (Ed.), Biography and society (pp. 515). Beverly Hills, California: Sage.

Bertaux, D. (Ed.). (1981). Biography and society. Beverly Hills: Sage.

Bertaux, D., \& Kohli, M. (1984). The life story approach: A continental view. In R. Turner \& J. Short (Eds.), Annual review of sociology (pp. 215-262). Palo Alto, California: Annual Reviews Inc.

Bevis, E., \& Murray, J. (1990). The essence of the curriculum revolution: Emancipatory teaching. Journal of Nursing Education, 29(7), 326-331.

Bodley, J. (1982). Victims of progress. Mountain View, California: Mayfield.

Bond, M., \& Jones, M. (1993). Cultural immersion: Student experiences in Mexico. IMPRINT(April/May), 66-68.

Bond, M., \& Jones, M. (1994). Short term cultural immersion in Mexico. Nursing and Health Care, 15(5), 248-253.

Borchet, C. (2001). Women and health: Mainstreaming the gender perspective into the health sector: Report of the expert group meeting 28 September-2 October 1998 Tunis (Tunisia). Journal of Government Information, 28, 457491.

Bowles, G., \& Klein, R. D. (Eds.). (1983). Theories of women's studies. London: Routledge \& Kegan Paul.

Bouchet-Saulnier, F. (2002). The practical guide to humanitarian law (L. Brav, Trans.). New York: Rowman \& Littlefield. 
Boud, D., Keogh, R., \& Walker, D. (Eds.). (1985). Reflection: Turning experience into learning. London: Kogan Page.

Boutain, D. (1999a). Critical language and discourse study: Their transformative relevance for critical nursing inquiry. Advances in nursing science, 21(3), $1-8$.

Boutain, D. (1999b). Critical nursing scholarship: Exploring critical social theory with African American studies. Advances in nursing science, 21(4), 37-47.

Boyd, E., \& Fales, A. (1983). Reflective learning: Key to learning from experience. Journal of Humanistic Psychology, 23(2), 99-117.

Bradshaw, D. (1999). Transforming lives, transforming communities: A conceptual framework for further education. Melbourne: Adult, Community and Further Education Board, Victoria.

Brause, R. (2000). Writing your doctoral dissertation. London: Falmer Press.

Braverman, P., Egerter, S., Cubbin, C., \& Marchi, K. (2004). An approach to studying social disparities in health and health care. Research and Practice, 94(12), 2139-2148.

Brettell, C. (1997). Blurred Genres and blended voices: Life history, biography, autobiography, and the auto/ethnography of women's lives. In Reed-Danahay (Ed.), Auto/ethnography. New York: Berg.

Brodzki, B., \& Schenck, C. (Eds.). (1988). Life/lines. Ithaca, New York: Cornell University Press.

Bronowski, J. (1965). Science and human values. New York: Harper \& Row.

Browner, C., \& Leslie, J. (1996). Women, work, and household: health in the context of development. In C. Sargent (Ed.), Gender and health (pp. 260275). Upper Saddle River: Prentice Hall.

Browning, E. B. (n.d.). Sonnet XLIII. Sonnets from the Portuguese. New York: Harper and Row.

Brueggemann, Walter. (1987). Hope within history. Atlanta: John Knox Press.

Buber, M. (1937/1970). I and thou (W. Kaufmann, Trans.). New York: Charles Scribner's Sons. 
Burford, G., \& Hudson, J. (Eds.). (2000). Family Group Conferencing. New York: Aldine De Gruyter.

Burger, J. (1987). Report from the frontier. London: Zed Books Ltd.

Burke, A., \& Shields, W. (2005). Millennium development goals: Slow movement threatens women's health in developing countries. Contraception, 72(4), 247249.

Burnard, P. (1991). Experiential learning in action. Avebury, England: Aldershot.

Burnard, P. (1993). Using experiential learning methods with larger groups of students. Nurse Education Today, 13, 60-65.

Burnard, P. (2005). Issues in helping students from other cultures. Nurse Education Today, 25(3), 176-180.

Button, L., Green, B., Tengnah, C., Johansson, I., \& Baker, C. (2005). The impact of international placements on nurses' personal and professional lives: literature review. Journal of Advanced Nursing, 50(3), 315-324.

Buvinic, M., Gwin, C., \& Bates, L. (1996). Investing in women: Progress and prospects for the World Bank. Washington, D.C.: World Bank.

Callister, L. (2004). Gaining a global perspective; the impact of international undergraduate clinical electives. Communication Nursing Research, 37, 361.

Callister, L., \& Hobbins-Garbett, D. (2000). "Enter to learn, go forth to serve": Service learning in nursing education. Journal of Professional Nursing, 16(3), 177-183.

Campesino, M. (2002). Commentary: Problematic issues in cultural comparisons. Journal of Professional Nursing, 18(6), 343-345.

Campinha-Bacote, J. (1999). Model and instrument for addressing cultural competence in health care. Journal of Nursing Education, 38(5), 203-207.

Campinha-Bacote, J. (2003). The process of cultural competence in the delivery of healthcare services. Cincinnati, Ohio: Transcultural C.A. R. E. Associates.

Cancian, F., \& Gordon, S. (1988). Changing emotion norms in marriage: love and anger in U. S. women's magazines since 1900. Gender and Society, 2, 308342. 
Cangelosi, P. (2005). Let's help them stay in nursing! Reflections on Nursing Leadership (Fourth quarter), 8-9.

Capera. (1983). The turning point. New York: Bantam Books.

Carpio, B., \& Majumdar, B. (1993). Experiential learning: An approach to transcultural education for nursing. Journal of Transcultural Nursing, 4(2), 4-11.

Carroll, T. (1983). Women, religion and developement in the third world. New York: Praeger.

Carryer, J. (1997). A feminist appraisal of the experience of embodied largeness. A challenge for nursing. Unpublished PhD, Victoria University, Wellington, New Zealand.

Carter, B. (2004). Faith, hope and clarity. AARP The Magazine, 47(6B), 54-61.

Carter, M. (1985). The philosophical dimensions of qualitative nursing science research. In M. Leininger (Ed.), Qualitative research methods in nursing (pp. 27-32). London: Grune \& Stratton.

Cattell, M. (1996). Gender, aging, and health: a comparative approach. In C. Sargent (Ed.), Gender and health: An international perspective (pp. 87-122). Upper Saddle River: Prentice Hall.

Cedar Crest hosts. (1996). Cedar Crest hosts Scandinavian nursing student exchange program. Pennsylvania Nurse, 51, 23-24.

Chalasinski, J. (1981). The life records of the young generation of Polish peasants as a manifestation of contemporary culture. In Bertaux (Ed.), Biography and society. Beverly Hills: Sage.

Challenges for. (2001). Challenges for the future: Wednesday's plenary summaries. Paper presented at the Healthy Women: Healthy World: 28th Annual Conference, Washington, D.C.

Chapman, R., \& Berggren, J. (2005). Radical contextualization: Contributions to an anthropology of racial/ethnic health disparitis. Health: An interdisciplinary Journal for the Social Study of Health, Illness and Medicine, 9(2), 145-167.

Chenitz, C., \& Swanson, J. (1986). From practice to grounded theory. Menlo Park, California: Addison-Wesley. 
Cheru, F. (1992). Structural adjustment, primary resource trade and sustainable development in Sub-Saharan Africa. World Development, 20(4), 497-512.

Chinn, P., \& Watson, J. (1994). Art \& aesthetics in nursing. New York: National league for Nursing Press.

Christ, C. (1980). Diving deep and surfacing. Boston: Beacon Press.

Church, K. (1995). Forbidden narratives (Vol. 2). Amsterdam: Gordon and Breach.

Clandinin, D., \& Connelly, F. (1994). Personal experience methods. In N. Denzin \& Y. Lincoln (Eds.), Handbook of qualitative research (pp. 413-427). Thousand Oaks: Sage.

Clark, C. (2005). Transforming nursing education: A partnership social system for alignment with philosophies of care. International Journal of Nursing Education Scholarship, 2(1), 1-18.

Coffey, M., \& Atkinson, P. (1996). Making sense of qualitative data. Thousand Oaks, California: Sage.

Colling, J., \& Wilson, T. (1998). Short-term reciprocal international academic exchange program. Journal of Nursing Education, 37(1), 34-36.

Cook, R., \& Dickens, B. (2001). Human rights to safe motherhood. International Journal of Gynecology \& Obstetrics, 76, 225-231.

Cook, R., Dickens, B., \& Syed, S. (2004). Obstetric fistula: The challenge to human rights. International Journal of Gynecology \& Obstetrics, 87, 72-77.

Cornelius, L. (2004). African Americans and access to health care: Trends in the use of health services. In I. Livingston (Ed.), Praeger handbook of Black American health (Vol. Two, pp. 701-715). Westport, Connecticut: Praeger.

Cotroneo, M., Grunzweig, W., \& Hollingsworth, A. (1986). All real living is meeting: The task of international education in a nursing curriculum. Jounal of Nursing Education, 25(9), 384-386.

Cox, R. (1980). Social forces, states and world orders: Beyond international relations theory. Journal of International Studies, 10(2), 126-155.

Craft, N. (1997). Women's health: women's health is a global issue. British Medical Journal, 315(7116), 1154-1157. 
Crampton, P., Salmond, C., Kirkpatrick, R., Scarborough, R., \& Skelly, C. (2000).

Degrees of deprivation in New Zealand: An atlas of socioeconomic difference. Auckland: David Bateman Ltd.

Craven, R. F., \& Hirnle, C. J. (2003). Fundamentals of nursing: Human health and function. Philadelphia: Lippincott, Williams and Wilkins.

Creswell, J. (1998). Qualitative inquiry and research design. Thousand Oaks: Sage.

Cummings, P. (1998). Nursing in Barbados: A fourth-year elective practice experience for nursing students and registered nurses. Journal of Nursing Education, 37(1), 42-44.

Currier, C., Omar, M., Talarczyk, G., \& Guerrero, R. (2000). Development and implementation of a semester program in Mexico for senior nursing students. Journal of Professional Nursing, 16(5), 293-299.

D'Aluisio, F., \& Menzel, P. (1996). Women in the material world. San Francisco: Sierra Club Books.

Davies, B. (1992). Women's subjectivite and feminist stories. In C. Ellis \& M. Flaherty (Eds.), Investigating subjectivity (pp. 53-78). Newbury Park: Sage.

Davis, K., \& Glass, N. (1999). Contemporary theories and contemporary nursing Advancing nursing care for those who are marginalised. Contemporary Nurse, 8 (2), 32-38.

Davis, G., \& Parker, C. (1997). Writing the doctoral dissertation: A systematic approach (2nd ed.). New York: Barron's.

Davis, P., \& Dew, K. (Eds.). (1999). Health and society in Aotearoa New Zealand. Auckland: Oxford University Press.

de Costa, C. (2001). Flora's legacy. The Lancet, 358, 2162-2163.

DeDee, L., \& Stewart, S. (2003). The effect of student participation in international study. Journal of Professional Nursing, 19(4), 237-242.

del Bueno, D., \& Vincent, P. (1986). Organizational culture: How important is it? Journal of Nursing Administration, 16, 15-20.

DeLuca, E. (2005). Crossing cultures: The lived experience of Jordanian graduate students in nursing: A qualitative study. International Journal of Nursing Studies, 42, 657-663. 
Denhardt, R. (1981). In the shadow of organization. Lawrence, Kansas: The Regents Press of Kansas.

Denzin, N. (1989a). Interpretive biography. Newbury Park: Sage.

Denzin, N. (1989b). Interpretive interactionism. Newbury Park: Sage.

Denzin, N., \& Lincoln, Y. (Eds.). (1994). Handbook of qualitative research. Thousand Oaks, CA: Sage.

DeSantis, L. (1999). Exploring transcultural communication as a participanobserver. In J. Luckman (Ed.), Transcultural communication in nursing. Boston: Delmar.

Devereux, G. (1967). From anxiety to method. The Hague: Mouton Press.

Dewey, J. (1916). Democracy in education. New York: Macmillan.

Diekelmann, N. (1990). Nursing education: Caring, dialogue, and practice. Journal of Nursing Education, 29(7), 300-305.

Diekelmann, N. (1993). Behavioral Pedagogy: A Heideggerian hermeneutical analysis of the lived experiences of students and teachers in baccalaureate nursing education. Journal of Nursing Education, 32(6), 245-254.

Diekelmann, N. (1995). Reawakening thinking: is traditional pedagogy nearing completion? Journal of Nursing Education, 34(5), 195-196.

Diekelmann, N. (2001). Narrative pedagogy: Heideggerian hermeneutical analyses of lived experiences of students, teachers, and clinicians. Advances in Nursing Science, 23(3), 53-71.

Diekelmann, N. (Ed.). (2003). Teaching the practitioners of care. Madison, Wisconsin: The University of Wisconsin Press.

Dilthey, W. (1910/1985). Poetry and experience (C. Rodie, Trans. Vol. V). Princeton, New Jersey: Princeton University Press.

Dodd, C. (1998). Dynamics of intercultural communication (5th ed.). Boston: McGraw Hill.

d'Oliveira, A., Diniz, S., \& Schraiber, L. (2002). Violenced against women in health -care institutions: An emerging problem. Lancet, 359(May 11), 1681-1685. 
Dollar, D., \& Gatti, R. (1999). Gender inequality, income and growth: are good times good for women? (Policy Research Report on Gender and Development.Working paper series, No. 1): The World Bank.

Dossey, B., Keegan, L., \& Guzzetta, C. (Eds.). (2000). Holistic nursing (3rd ed.). Maryland, USA: Aspen.

Douglass, B., \& Moustakas, C. (1985). Heuristic Inquiry. Journal of Humanistic Psychology, 25(3), 39-55.

Drife, J., \& Kunzel, W. (2005). Editor's highlights. European Journal of Obstetrics \& Gynecology and Reproductive Biology, 12(1), 1-2.

DuBois, C. (1963). The curriculum in cultural anthropology. In D. Mandelbaum, Lasker, G., \& Albert, E. (Ed.), The teaching of anthropology. (pp. 27-38). Washington, DC: American Anthropological Association.

Duffy, M., Harju, L., Huittinen, L., \& Trayner, C. (1999). An innovative model for international undergraduate education. Nursing and Health Care Perspectives,

Durie, M. (1994). Whaiora. Auckland: Oxford University Press.

Durie, M. (2001). Mauri ora: The dynamics of Maori health. Auckland: Oxford University Press.

Eliot, T. S. (1943). Four Quartets. New York: Harcourt, Brace, and World.

Ellis, C., \& Flaherty, M. (1992). An agenda for the interpretation of lived experience. In C. Ellis \& M. Flaherty (Eds.), Investigating subjectivity (pp. 116). Newbury Park: Sage.

Ellis, C., \& Flaherty, M. (Eds.). (1992). Investigating subjectivity. Newbury Park: Sage.

Engebretson, J., \& Headley, J. (2000). Cultural diversity and care. In B. Dossey, L. Keegan \& C. Guzzetta (Eds.), Holistic nursing. Maryland, USA: Aspen.

Enloe, C. (1990). Making feminist sense of international politics. Berkeley, California: University of California Press.

Epp, J., \& Ringheim, K. (2004). Banking on reproductive health. Washington, D.C.: Global Health Council. 
Evans, D. (1996). How to write a better thesis report. Melbourne: Melbourne University Press.

Evanson, T., \& Zust, B. (2004). The meaning of participation in an international service experience among Baccalaureate nursing students. International Journal of Nursing Education Scholarship, 1(1), 1-14.

Fahrenwald, N., Bassett, S., Tschetter, L., Carson, P., White, L., \& Winterboer, V. (2005). Teaching core nursing values. Journal of Professional Nursing, 21(1), 46-51.

Fennal, M., Fair, N., Fleming, K., Gibson, S., Hugee, B., \& Winborne-Tanner, M. (1998). A clinical experience in cultural diversity. The South Carolina Nurse, 15-18.

Fest, G. (2003a). Shining light: The lady behind the lamp: Part I. Nurse Week (6 October), 14-16.

Fest, G. (2003b). Shining light: The lady behind the lamp: Part II. Nurse Week (20 October), 14-17.

Fikree, F., Razzak, J., \& Durocher, J. (in press). Attitudes of Pakistani men to domestic violence: A study from Karachi, Pakistan. The Journal of Men's Health \& Gender, In press.

Filmer, D., Hammer, J., \& Pritchett, L. (2000). Weak links in the chain: A diagnosis of health policy in poor countries. The World Bank Research Observer, 15(2), 199- 224.

Filmer, D., \& Pritchett, L. (1998). The effect of household wealth on education attainment around the world: demographic and health survey evidence (World Bank Policy Research Working Paper No. 1980, September 1998): World Bank Research.

Flexner, S. (Ed.). (1983). Random house dictionary of the English language (2nd ed.). New York: Random House.

Foden, G. (1999). Ladysmith. New York: Random House.

Foliaki, S., \& Pearce, N. (2003). Changing pattern of ill health for indigenous people. British Medical Journal, 327, 407-408. 
Fotso, J., \& Kuate-Defo, B. (2005). Socioeconomic inequalities in early childhood malnutrition and morbidity: Modification of the household-level effects by the Community SES. Health \& Place, 11, 205-225.

Francis, D. (2004). Reconstructing the meaning given to critical incidents in nurse education. Nurse Education in Practice, 4(4), 244-249.

Frankl, V. (1969). Self-transcendence as a human phenomenon. In A. Sutich \& M. Vich (Eds.), Readings in humanistic psychology (pp. 113-125). London: Collier-Macmillan.

Freedman, D., Frey, O., \& Zauhar, F. (Eds.). (1993). The intimate critique. Durham: Duke University Press.

Freire, P. (1972). Pedagogy of the oppressed. Harmondsworth: Penquin.

Freire, P. (1998). Teachers as cultural workers (D. Macedo, D. Koiki, \& A.Oliveira, Trans.). Boulder, Colorado: Westview Press.

Frey, O. (1993). Beyond literary Darwinism: Women's voices and critical discourse. In D. Freedman, O. Frey \& F. Zauhar (Eds.), The intimate critique. Durham: Durham University Press.

Frick, W. (1983). The symbolic growth experience. Journal of Humanistic Psychology, 23(1), 108-125.

Frick, W. (1987). The symbolic growth experience: Paradigm for a humanisticexistential learning theory. Journal of Humanistic Psychology, 27(4), 406423.

Frick, W. (1990). The symbolic growth experience: A chronicle of heuristic inquiry and a quest for synthesis. Journal of Humanistic Psychology, 30(1), 64-80.

Frisch, N. (1990). An international nursing student exchange program: An educational experience that enhanced student cognitive development. Journal of Nursing

Frost, R. (1921). Mountain Interval. New York: Henry Holt.

Fuse, K., \& Crenshaw, E. (2006). Gender imbalance in infant mortality: A crossnational study of social structure and female infanticide. Social Science \& Medicine, 62(2), 360-374. 
Gadamer, H. (1960/1982). Truth and method (T. G.Barden \& J.Cumming, Trans.). New York: Crossroads.

Gadamer, H. (1996/2000). The beginning of philosophy (R.Coltman, Trans.). New York: Continuum Publishing Company.

Galeo, S., Freudenberg, N., \& Vlahov, D. (2004). Cites and population health. Social Science \& Medicine, 60(5), 17-33.

Gash, S. (2000). Effective literature searching for research (second ed.). Hampshire: Gower.

Gates, H. (1993). "What's in a name?”: Some meanings of blackness. In D. Freedman, O. Frey \& F. Zauhar (Eds.), The intimate critique. Durham: Durham University Press.

Gennaro, S. (2005). Overview of current state of research on pregnancy outcomes in minority populations. American Journal of Obstetrics and Gynecology, 192, S3-S10.

Georges, J. (2003). An emerging discourse. Advances in nursing science, 26(1), 4452.

Germain, A. (2004). Reproductive health and human rights. The Lancet, 363(9402), 65-66.

Gershman, J., \& Irwin, A. (2000). Getting a grip on the global economy. In J. Y. Kim, J. Millen, A. Irwin \& J. Gershman (Eds.), Dying for growth (pp. 1143). Maine: Common Courage Press.

Geertz, C. (1973). The interpretation of cultures. New York: Basic Books.

Giele, J., \& Elder, G. (Eds.). (1998). Methods of life course research. Thousand Oaks, CA: Sage.

Gillis, A., \& Jackson, W. (2002). Research for nurses. Philadelphia: F.A. Davis Company.

Glaser, B., \& Strauss, A. (1967). The discovery of grounded theory. Chicago: Aldine.

Goffman, E. (1959). The presentation of self in everyday life. Garden City: Doubleday. 
Goldberg, L., \& Brancato, V. (1998). A United Kingdom nursing student partnership. Nurse Educator, 23(5), 30-34.

Goodenough, W. (1964). Cultural anthropology and linguistics. In D. Hymes (Ed.), Language in culture and society (pp. 36-39). New York: Harper \& Row, Publishers.

Goodenough, W. (1981). Culture, language, and society. Menlo Park, California: The Benjamin/Cummings.

Goodman, J. (1998). Ideology and critical ethnography. In G. Shacklock \& J. Smyth (Eds.), Being reflexive in critical educational and social research (pp. 5066). London: Falmer Press.

Gordon, J. A. (2002). Beyond the classroom walls. London: Routledge Falmer.

Graham, L. (1992). Archival research in intertextual analysis: Four representations of the life of Dr. Lillian Moller Gilbreth. In C. Ellis \& M. Flaherty (Eds.), Investigating subjectivity (pp. 17-30). Newbury Park: Sage.

Graham, W., \& Hussein, J. (2004). The right to count. The Lancet, 363(9402), 6768.

Grant, E., \& McKenna, L. (2003). International clinical placements for undergraduate students. Journal of Clinical Nursing, 12, 529-535.

Graves, M. (2003). Lessons learned from valuable international experience. Kentucky Nurse, 51(3), 19-20.

Grene, M. (Ed.). (1969). Knowing and being. Chicago: Chicago University Press.

Grown, C., Gupta, G., \& Pande, R. (2005). Taking action to improve women's health through gender equality and women's empowerment. The Lancet, 365(9458), 541-543.

Guba, E. (1981). Criteria for assessing the trustworthiness of naturalistic inquiries. Educational Resources Information Center Annual Review Paper, 29, 75-91. Gubrium, J., \& Holstein, J. (2002). From the individual interview to the interview society. In J. Gubrium \& J. Holstein (Eds.), Handbook of interview research (pp. 3-32). Thousand Oaks: Sage.

Gubrium, J., \& Holstein, J. (Eds.). (2002). Handbook of interview research. Thousand Oaks: Sage. 
Habermas, J. (1968/1971). Knowledge and human interests (J. Shapiro, Trans.). Boston: Beacon Press.

Hadwiger, M., \& Hadwiger, S. (1998). Transcultural Nursing Experience in the Philippines. Nurse Educator, 24(1), 12-15.

Hagerty, B., \& Patusky, K. (2003). Reconceptualizing the nurse-patient relationship. Journal of Nursing Scholarship, 35(2), 145-150.

Haloburdo, E., \& Thompson, M. (1998). A comparison of international learning experiences for baccalaureate nursing students: Developed and developing countries. Journal of Nursing Education, 37(1), 13-21.

Hamilton, A. (1990). Writing dissertations. London: RIBA.

Harry, V. (1996). Volitional change in elementary teachers' conceptions of science pedagogy via a generative learning model of training. (Doctoral dissertation, Pennsylvania State University, State College, Pennsylvania, 1996).

Dissertation Abstracts International, 57, 04A, 1467.

Hartrick, G. (1998). A critical pedagogy for family nursing. Journal of Nursing Education, 37(2), 80-84.

Haselgrave, M. (1997). What she wants. The Lancet, 349 Supplement I(S1-S2), s11s12.

Henningfeld, D. (2004). Putting the heart before the course: Passion vs. planning. Thought \& Action, $x x(1), 49-56$.

Hertz, R. (Ed.). (1997). Reflexivity \& voice. Thousand Oaks: Sage Publications.

Heuer, L., \& Bengiamin, M. (2001). American nursing students experience shock during a short-term international program. Journal of Cultural Diversity, 8(4), 128-134.

Heuer, L., Russell, L., \& Kahlstorf, H. (1997). Short-term cultural immersion in Russia. Prairie-Rose, 66(1), 7-11.

Hikins, J. (1989). Intrapersonal discourse and its relationship to human communication: Rhetorical dimensions of self-talk. In C. Roberts, K. Watson \& L. Barker (Eds.), Intrapersonal communication processes. New Orleans: SPECTRA. 
Hinds, P., Chaves, D., \& Cypess, S. (1992). Context as a source of meaning and understanding. In J. Morse (Ed.), Qualitative health research (pp. 31-42). Newbury Park: Sage.

Holloway, I., \& Wheeler, S. (1996). Qualitative research for nurses. London: Blackwell Science.

Holm, G. (2001). Violence against women: A global epidemic. The Danish Nursing Journal, 101, 8-11.

Holstege, M. (2000). The lived experience of student nurses providing health care relief to hurricane Mitch victims in Nicaragua. Unpublished Master of Science in Nursing, Grand Valley State University.

Holstein, J., \& Gubrium, J. (2000). The self we live by. New York: Oxford University Press.

Holt, G. (1998). A guide to successful dissertations study for students of the built environment (2nd ed.). University of Wolverhampton. West Midlands.: The Built Environment Research Unit School of Engineering and the Built Environment.

hooks, b. (2003). Teaching community. London: Routledge.

Hopkins, G. M. (1918/1948). Poems of Gerard Manley Hopkins (3rd ed.). London: Oxford University Press.

Howard, A., Ford, C., \& Mclean, H. (2005). Disparities in medication use, action, and prescribing for African Americans. In I. Livingston (Ed.), Praeger handbook of Black American health (Vol. Two, pp. 497-515). Westport, Connecticut: Praeger.

Hudson, W., \& Kane, J. (Eds.). (2000). Rethinking Australian citizenship. Cambridge: Cambridge University Press.

Huff, R., \& Kline, M. (Eds.). (1999). Promoting health in multicultural populations. Thousand Oaks, California: Sage.

Hurlock, D. (2003). Possibilities of a poetic pedagogy: The movement by which a life gets changed for keeps" (Bronwyn Wallace). (Doctoral dissertation, University of Calgary, Canada, 2003). Dissertation Abstracts International, 65, 01A, 96. 
Husserl, E. (1913/1969). Ideas (B. Gibson, Trans.). London: George Allen \& Unwin.

Hymes, D. (Ed.). (1964). Language in culture and society. New York: Harper \& Row, Publishers.

Inglis, A., Rolls, C., \& Kristy, S. (1998). The impact of participation in a study abroad programme on students' conceptual understanding of community health nursing in a developing country. Journal of Advanced Nursing, 28(4), 911-917.

Inglis, A., Rolls, C., \& Kristy, S. (2000). The impact on attitudes towards cultural difference of participation in a health focused study abroad program. Contemporary Nurse, 9(3/4), 246-255.

Intrator, S. (Ed.). (2002). Stories of the courage to teach. San Francisco: JosseyBass.

Ironside, P. (2001). Creating a research base for nursing education: An interpretive review of conventional, critical, feminist, postmodern, and phenomenologic pedagogies. Advances in nursing science, 23(3), 72-87.

Ironside, P. (2004). "Covering content" and teaching thinking: Deconstructing the additive curriculum. Journal of Nursing Education, 43(1), 5-12.

Islam, A., \& Tahir, M. (2002). Health sector reform in South Asia: new challenges and constraints. Health Policy, (60), 151-169.

Izard, C. (1991). The psychology of emotions. New York: Plenum Press.

Jackson, M., \& Jackson, R. (2002). Courage to teach: A retreat program of personal and professional renewal for educators. In S. Intrator (Ed.), Stories of the courage to teach (pp. 282-308). San Francisco: Jossey-Bass.

Jacobs, B., Fontana, J., Hidalgo, M., Matarese, D., \& Chinn, P. (2005). An emancipatory study of contemporary nursing practiced. Nursing Outlook, 53(1), 6-14.

James, V., \& Gabe, J. (Eds.). (1996). Health and the sociology of emotions. Oxford: Blackwell.

Jandt, F. (2001). Intercultural communication (3rd ed.). Thousand Oaks: Sage. 
Jiggins, J. (1989). How poor women earn income in Sub-Saharan Africa and what works against them. World Development, 17(7), 953-963.

Johns, C., \& Freshwater, D. (Eds.). (1998). Transforming nursing through reflective practice. London: Blackwell Science.

Johns, C., \& McCormack, B. (1998). Voice as a metaphor for transformation through reflection. In C. Johns \& D. Freshwater (Eds.), Transforming nursing through reflective practice. London: Blackwell Science.

Johnson, J. (1994). Intrapersonal spoken language: An attribute of extrapersonal competency. In D. Vocate (Ed.), Intrapersonal communication (pp. 169192). Hillsdale: Lawrence Erlbaum Associates.

Johnstone, M. (1999). Reflective topical autobiography: an under utilised interpretive research method in nursing. Collegian, 6(1), 24-29.

Jourard, S. (1967). To be or not to be... Gainesville, Florida: University of Florida Press.

Jourard, S. (1971). Self-disclosure: An experimental analysis of the transparent self. New York: Wiley-Interscience.

Kadar, M. (1992a). Whose life is it anyway? In M. Kadar (Ed.), Essays on life writing: From genre to critical practice. Toronto: University of Toronto Press.

Kadar, M. (Ed.). (1992b). Essays on life writing: from genre to critical practice. Toronto: University of Toronto Press.

Kalantzis, M. (2000). Multicultural citizenship. In W. Hudson \& J. Kane (Eds.), Rethinking Australian citizenship (pp. 99-110). Sydney: Cambridge University Press.

Kanhai-Brunton, R. (1993). The crippling of the third world: Shiva Naipul's heritage. In D. Freedman, O. Frey \& F. Zauhar (Eds.), The intimate critique. Durham: Duke University Press.

Kanpol, B. (1998). Where was I? Or was I? In G. Shacklock \& J. Smyth (Eds.), Being reflexive in critical educational and social research. London: Falmer Press. 
Katz, A. (2005). "Caregiving" is Cultural. Journal of Professional Nursing, 21(3), 139-140.

Kendall, G., \& Li, J. (2005). Early childhood socialization and social gradients in adult health: A commentary on Singh-Manoux and Marmot's "Role of socialization in explaining social inequalities in health. Social Science \& Medicine, 61(11), 2272-2276.

Ketefian, S., \& Porter, C. (2000). Diversity in nursing education-Part III. Journal of Professional Nursing, 16(3), 129.

Kielhofner, G. (1982). Qualitative research: Part one-paradigmatic grounds and issues of reliability and validity. The Occupational Therapy Journal of Research, 2(2), 67-79.

Kierkegaard, S. (1846/1941). Concluding unscientific postscript (D. Swenson \& W. Lowrie, Trans.). Princeton, New Jersey: Princeton University Press.

Kierkegaard, S. (1851/1941). For self-examination and judge for yourselves! (W. Lowrie, Trans.). Princeton: Princeton University Press.

Kim, J. Y., Millen, J., Irwin, A., \& Gershman, J. (Eds.). (2000). Dying for growth. Maine: Common Courage Press.

Kinchloe, J., \& Steinberg, S. (Eds.). (1998). Unauthorized methods: Strategiesfor critical teaching. New York: Routledge.

King, M., \& O'Boyle. (2002). Idea and value exchange worldwide: American culture in the world. Journal of American \& Comparitive Cultures, 25(Spring), 1-8.

Koithan, M. (1994). Incorporating multiple modes of awareness in nursing curriculum. In Art \& aesthetics in nursing. (pp. 145-162). New York: National League for Nursing.

Kolb, D. (1984). Experiential Learning. New Jersey: Prentice-Hall.

Kollar, S., \& Ailinger, R. (2002). International clinical experiences: Long-term impact on students. Nurse Educator, 27(1), 28-31.

Krefting, L. (1991). Rigor in qualitative research: The assessment of trustworthiness. American Journal of Occupational Therapy, 45(3), 214-222.

Kress, G. (1995). Writing the future. Sheffield: NATE.

Kroeber, A. (1952). The nature of culture. Chicago: University of Chicage Press. 
Kroeber, A., \& Kluckhohn, C. (1952). Culture: A critical review of concepts and definitions. Cambridge, Massachusett: Peabody Museum of American Archaeology and Ethnology, Harvard University.

Kunzman, R. (2002). The meaning of life assignment. In S. Intrator (Ed.), Stories of the courage to teach (pp. 84-93). San Francisco: Jossey-Bass.

Kurland, J. (2000). Public health in the new millennium II: Social exclusion (No. A66449539). Washington, D.C.

Langness, L., \& Frank, G. (1981). Lives. Novato, California: Chandler \& Sharp Publishers, Inc.

Lauterbach, S. (2002). In another world: five years later-a phenomenological nursing inquiry into meanings, "essences" of mothers' lived experience with perinatal death of a wished -for baby unfolding over time. International Journal for Human Caring, 6(1), 17-24.

Lawn, J., Cousens, S., \& Zupan, J. (2005). Four million neonatal deaths: When? Where? Why? The Lancet, 365(9462), 891-900.

LeCompte, M., \& Preissle, J. (1993). Ethnography and qualitative design in educational research (2nd ed.). New York: Academic Press.

Lee, N. (1997). Learning from abroad: The benefits for nursing. Journal of Nursing Management, 5, 359-365.

Lee, N. (2004). The impact of international experience on student nurses' personal and professional development. International Nursing Review, 51, 113-122.

Leenerts, M. (2003). Teaching personal knowledge as a way of knowing self in therapeutic relationship. Nursing Outlook, 51(4), 158-164.

Leh, S., Robb, W., \& Albin, B. (2004). Responding to the challenge of developing a global perspective in nursing education. Nursing Education Perspectives, 25(2), 86-90.

Leininger, M. (1978). Transcultural nursing: Concepts, theories and practices. New York: John Wiley and Sons.

Leininger, M. (1979). Transcultural nursing. USA: Masson Publishing USA. 
Leininger, M. (1985a). Audiovisual methods in nursing research. In M. Leininger (Ed.), Qualitative research methods in nursing (pp. 331-342). London: Grune \& Stratton.

Leininger, M. (1985b). Nature, rationale, and importance of qualitative research methods in nursing. In M. Leininger (Ed.), Qualitative research methods in nursing (pp. 1-27). Orlando: Grune \& Stratton, Inc.

Leininger, M. (Ed.). (1985). Qualitative Research Methods in Nursing. Orlando: Grune \& Stratton, Inc.

Leininger, M. (2002). Culture care assessment for congruent competency practices. In M. Leininger, \& McFarland, M. (Ed.), Transcultural nursing: Concepts,theories, research \& practice (3rd ed., pp. 117-144). New York: McGraw-Hill.

Leininger, M., \& McFarland, M. (Eds.). (2002). Transcultural nursing: Concepts,theories, research \& practice (3rd ed.). New York: McGraw-Hill.

Leo, J. (2002, July 22). Professors who see no evil. U.S. News \& World Report, 133, 14.

LeVasseur, J. (1999). Toward an understanding of art in nursing. Advances in nursing science, 21(4), 48-63.

Levine, M. A. (1977). Cultural marginality and health innovativeness. Unpublished master's thesis, Pennsylvania State University, State College, Pennsylvania.

Levine, M. A. (1991). Teaching the peasant midwife in rural Honduras. Midwives Chronicle, 104(1240), 142-143.

Levine, M. A. (1994). The relationship of poverty to pregnancy in the developing world. Midwives Chronicle, 107(1275), 118-121.

Levine, M. A. (2000a). Childbirth and midwifery in Nepal: Part 1. Journal of the International Confederation of Midwives, 13(4), 6-8.

Levine, M.A. (2000b). Childbirth and midwifery in Nepal: Part 2. Journal of the International Confederation of Midwives, 13(5), 8-9.

Levine, M.A. (2001). Childbearing women and poverty in the developing world. New Zealand College of Midwives Journal (24), 4-6. 
Levine, M. A., Perpetua, E., Allen, K., Comer, L., Lindsley, C., Loeffler, D., et al. (2002). Transcultural midwifery: US students visit and work in Far East Russia. International Midwifery, 15(5), 6-8.

Lim, S. (1993). The scarlet brewer and the voice of the colonized. In D. Freedman, O. Frey \& F. Zauhar (Eds.), The intimate critique. Durham: Duke University Press.

Lincoln, Y., \& Guba, E. (1985). Naturalistic Inquiry. London: Sage.

Lindquist, G. (1984). A cross-cultural experience: Comparative study in nursing and health care. Journal of Nursing Education, 23(May), 212-214.

Linton, R. (1955). The tree of culture. New York: Alfred A. Knopf.

Livingston, I. (Ed.). (2004a). Praeger handbook of Black American health (2nd ed. Vol.1). Westport, Connecticut: Praeger Publishers.

Livingston, I. (Ed.). (2004b). Praeger handbook of Black American health (2nd ed. Vol. 2). Westport, Connecticut: Praeger Publishers.

Lopez-Alonso. (2001). La globalización y las salud en los groupos minoritarios. Cultura de los Cuidados, 5(10), 63-71.

Lorde, A. (1984). Sister outsider. Freedom, California: The Crossing Press.

Luckman, J. (Ed.). (1999). Transcultural communication in nursing. Boston: Delmar Publishers.

Ludwig, A. (1997). How do we know who we are? Oxford: Oxford University Press.

Mandelbaum, D., Lasker, G., \& Albert, E. (Eds.). (1963). The teaching of anthropology. Washinton, DC: American Anthropological Association.

Mann, B. (1972). A little gem: Ethnography of an urban jewelry store. In J. Spradley \& D. McCurdy (Eds.), The cultural experience. Chicago: Science Research Associates.

Mariko, M. (2003). Quality of care and the demand for health services in Bamako, Mali: The specific roles of structural, process, and outcome components. Social Science \& Medicine, 56(6), 1183-1196.

Massiah, J. (1989). Women's lives and livelihoods: a view from the Commonwealth Caribbean. World Development, 17(7), 965-977. 
Mavalankar, D., \& Rosenfield, A. (2005). Maternal mortality in rosource-poor settings: Policy barriers to care. American Journal of Public Health, 95(2), 200-203.

Maze, C. (2005). Registered nurses' personal rights vs. professional responsibility in caring for members of underserved and disenfranchised populations. Journal of Clinical Nursing, 14, 546-554.

McAuliffe, M., \& Cohen, M. (2005). International nursing research and educational exchanges: A review of the literature. Nursing Outlook, 53(1), 21-25.

McEldowney, R. (2003). Shape-shifting: Stories of teaching for social change in nursing. Unpublished doctoral dissertation, Victoria University, Wellington, New Zealand.

McGee, P. (1992). Teaching transcultural care. London: Chapman \& Hall.

McLuhan, T. C. (1971). Touch the earth. New York: Promontory Press.

Mead, G. (1967). Mind, self and society. Chicago: The University of Chicago Press.

Mehrotra, S., \& Jarrett, S. (2002). Improving basic health service delivery in lowincome countries: 'voice' to the poor. Social Science \& Medicine, 54(11), $1685-1690$.

Meleis, A. (1987). ReVisions in knowledge development: A passion for substance. Scholarly Inquiry for Nursing Practice: An International Journal, 1(1).

Meleis, A. (1997). Theoretical Nursing. New York: Lippincott.

Meleis, A. (2001). Small steps and giant hopes: Violence on women is more than wife battering. Health Care for Women International, 23, 313-315.

Meleis, A., \& Lindgren, T. (2001). Show me a woman who does not work! Journal of Nursing Scholarship, 33(3), 209-210.

Mezirow, J. and Associates. (2000). Learning as transformation. San Francisco: Jossey-Bass.

Mhlanga, R. (2003). Abortion: Developments and impact in South Africa. British Medical Bulletin, 67, 115-126.

Miles, M., \& Huberman, A. (1994). Qualitative data analysis (2 ed.). Thousand Oaks: Sage.

Mills, C. W. (1959). The sociological imagination. London: Oxford Press. 
Minnich, E. (1990). Transforming knowledge. Philadelphia: Temple University Press.

Mishler, E. (1986). Research interviewing: Context and narrative. Cambridge, MA.: Harvard University Press.

Morris, L. (1999). No wealth no health: Access to healthcare for low-income women with children. Whitireia Nursing Journal(6), 43-50.

Morris, W. (Ed.). (1976). The American heritage dictionary of the English language. Boston: Houghton Mifflin.

Morse, J. (1997). Recognizing the power of qualitative research. In J. Morse (Ed.), Completing a qualitative project (pp. 1-7). Thousand Oaks: Sage.

Morse, J. (2001). Situating grounded theory within qualitative inquiry. In R. Scheiber \& P. N. Stern (Eds.), Using grounded theory in nursing (pp. 1-16). New York: Springer.

Morse, J. (Ed.). (1992). Qualitative health research. Newbury Park: Sage.

Morse, J. (Ed.). (1997). Completing a qualitative project. Thousand Oaks: Sage.

Moser, C. (1989). Gender planning in the third world: meeting practical and strategic gender needs. World Development, 17(11), 1799-1825.

Moss, N. (2002). Gender equity and socioeconomic inequality: A framework for the patterning of women's health. Social Science \& Medicine, 54, 649-661.

Moustakas, C. (1956). True experience and the self. In C. Moustakas (Ed.), The self. New York: Harper \& Row.

Moustakas, C. (1959). The alive and growing teacher. New York: Philosophical Library.

Moustakas, C. (1961). Loneliness. USA: Prentice-Hall.

Moustakas, C. (1966). The authentic teacher. Cambridge, MA: Howard A. Doyle.

Moustakas, C. (1967). Creativity and conformity. New York: D. Van Nostrand.

Moustakas, C. (1972). Loneliness and love. Englewood Cliffs: Prentice-Hall.

Moustakas, C. (1975). The touch of loneliness. Englewood Cliffs: Prentice-Hall.

Moustakas, C. (1977). Turning Points. Englewood Cliffs: Prentice-Hall.

Moustakas, C. (1981). Heuristic research. In P. Reason \& J. Rowan (Eds.), Human inquiry (pp. 207-218). New York: John Wiley \& Sons. 
Moustakas, C. (1990). Heuristic research. Newbury Park: Sage.

Moustakas, C. (Ed.). (1956). The self. New York: Harper \& Row.

Moustakas, C., \& Perry, C. (1973). Learning to be free. London: Prentice-Hall International.

Mumtaz, Z., \& Salway, S. (2005). 'I never go anywhere': Extricating the links between women's mobility and uptake of reproductive health srvices in Pakistan. Social Science \& Medicine, 60, 1751-1765.

Munhall, P., \& Oiler, C. (1986). Nursing research: A qualitative perspective.

Norwalk, Connecticut: Appleton-Century-Crofts.

Murphy, B., \& Dillon, C. (1998). Interviewing in action. Boston: Brooks/Cole.

Nanus, B. (1992). Visionary leadership. San Francisco: Jossey-Bass.

National Health Committee. (1998). The social, cultural and economic determinants of health in New Zealand: Action to improve health. Wellington: National Advisory Committee on Health and Disability.

Newman, M. (2003). A world of no boundaries. Advances in Nursing Science, 26(4), 240-245.

Nieswiadomy, R. (2002). Foundations of nursing research (4th ed.). Upper Saddle River, New Jersey: Prentice Hall.

Nieto, S., \& Gordon, S. (2002). Adult conversations about unasked questions: Teaching for educational justice. In S. Intrator (Ed.), Stories of the courage to teach (pp. 64-78). San Francisco: Jossey-Bass.

Nightingale, F. (1859/1980). Notes on nursing: What it is and what it is not. New York: Churchill Livingstone.

O'Brien, M. (1981). The politics of reprodution. London: Routledge \& Kegan Paul.

Okojie, C. (1994). Gender inequalities of health in the third world. Social Science \& Medicine, 39(9), 1237-1247.

Olbrys, S. (2004). 'Keep your keys': Teaching, democracy, and performance art. Thought and Action, xx(1), 9-22.

O'Rand, A. (1998). The craft of life course studies. In J. Giele \& G. Elder (Eds.), Methods of life course research (pp. 52-74). Sage Publications. 
Packard, M. (2004). Unfolding the blanket of understanding in the listening space: A phenomenological, exploration of 'being-with' in the nursing student-teacher relationship. Unpublished PhD, University of Maryland, Baltimore, Maryland.

Pallitto, C., \& O'Campo, P. (2005). Community level effects of gender inequality on intimate partner violence and unintended pregnancy in Colombia: Testing the feminist perspective. Social Science \& Medicine, 60(10), 2205-2216.

Parse, R. (2001). Qualitative Inquiry: The path of sciencing. Boston: Jones and Bartlett.

Parse, R., R., Coyne, A. B., \& Smith, M. J. (1985). Nursing research: qualitative methods. Bowie, Md.: Brady Communications.

Paterson, B. (1991). The juggling act: An ethnographic analysis of clinical teaching in nursing education. (Doctoral dissertation, The University of Manitoba, 1991). Dissertation Abstracts International, 54, 03B, 1310.

Paterson, J., \& Zderad, L. (1976). Humanistic nursing. New York: Wiley \& Sons.

Patton, M. (1990). Qualitative evaluation and research methods (2nd ed.). Newbury Park: Sage.

Peel, K. (1998). Incorporating international nursing in the curriculum: One baccalaureate program's experience. The South Carolina Nurse, July, August, September, 17-19.

Pelose, G. (1989). Metacognition as an intrapersonal communication process: The purposes of cognitive monitoring and methodology for its assessment. In C. Roberts, K. Watson \& L. Barker (Eds.), Intrapersonal communication processes (pp. 135-165). New Orleans: SPECTRA.

Peplau, H. (1952). Interpersonal relations in nursing. New York: Putnam's Sons. Perry, W. G. (1970). Forms of intellectual and ethical development in the college years. New York: Holt, Rinehart \& Winston.

Phillips, E., \& Pugh, D. S. (1998). How to get a PhD (2nd ed.). Bristol, Pa: Open University Press. 
Pick, W., Ross, M., \& Dada, Y. (2002). The reproductive and occupational health of women street vendors in Johannesburg, South Africa. Social Science \& Medicine, 54, 192-204.

Pillai, V., \& Gupta, R. (2006). Cross-national analysis of a model of reproductive health in developing countries. Social Science Research, 35(1), 210-227.

Plummer, K. (1983). Documents of life. London: George Allen \& Unwin.

Poincaré, H. (1901/1913). The foundations of science (G. Halsted, Trans.). Lancaster, Pennsylvania: The Science Press.

Polanyi, M. (1959). The study of man. Chicago: The University of Chicago Press. Polanyi, M. (1962). Personal knowledge. Chicago: The University of Chicago Press. Polanyi, M. (1966). The tacit dimension. Garden City: Doubleday \&Company, Inc. Polanyi, M. (1969). Knowing and being. In M. Grene (Ed.), Knowing and being. Chicago: University of Chicago Press.

Polit, D., Beck, C., \& Hungler, B. (2001). Essentials of nursing research (5 ed.). Philadelphia: Lippincott.

Polkinghorne, D. (1982). What makes research humanistic. Journal of Humanistic Psychology, 22(3), 47-54.

Polkinghorne, D. (1988). Narrative knowing and the human sciences. Albany: State University of New York Press.

Popkewitz, T., \& Brennan, M. (1998). Foucault's challenge: Discourse, knowledge and power in education. New York: Teachers College Press.

Potgieter, E. (2003). Relationship between the whole brain creativity model and Kolb's experiential learning model. Curationis, 26(3), 42-51.

Potter, P., \& Guzzetta, C. (2000). The holistic caring process. In B. Dossey, L. Keegan \& C. Guzzetta (Eds.), Holistic nursing (pp. 315-343). Maryland: Aspen.

Powdermaker, H. (1966). Stranger and friend. New York: Norton.

Powell, J. (1985). Autobiographical learning. In D. Boud, R. Keogh \& D. Walker (Eds.), Reflection: Turning experience into learning. London: Kogan Page. Powers, B., \& Knapp, T. (1995). A dictionary of nursing theory and research (2nd ed.). Thousand Oaks: Sage. 
Poverty is pronounced. (2001). World development report 2000/2001: Attacking poverty. New York: Oxford University Press.

Pross, E. (2000). International education experiences of baccalaureate nursing students. (Doctoral dissertation, University of Colorado, 2000). Dissertation Abstracts International, 61, 10B, 5239.

Pross, E. (2003). International nursing students a phenomenological perspective. Nurse Education Today, 23(6), 396-403.

Purnell, L. (1998). University of Delaware nursing students go to Panama. Delaware Nurses Association, 23(December 1998, January 1999), 20-23.

Purnell, L., \& Paulanka, B. (2003). Transcultural health care (2nd ed.). Philadelphia: F.A. Davis.

Quotations (n.d.). Retrieved April 19, 2006, from http://www.quotationspage.com/quotes /Martin_Luther_King_Jr. /

Ramsden, I. (1990). Kawa Whakaruruhau: Cultural safety in nursing education in Aotearoa. Wellington: Ministry of Education.

Ramsden, I. (1995). Cultural safety: implementing the concept. New Zealand College of Midwives Journal (7), 6-9

Ramsden, I. (1996). Cultural safety. Wellington, New Zealand: Nursing and midwifery education.

Ramsden, I. (2000). Cultural Safety/ Kawa whakaruruhau ten years on: a personal overview. Nursing Praxis in New Zealand, 15(1), 4-12.

Ramsden, I. (2002). Cultural safety and nursing education in Aotearoa and Te Waipounamu. Unpublished PhD, Victoria University, Wellington.

Ray, M. (1985). A philosophical method to study nursing phenomena. In M. Leininger (Ed.), Qualitative research methods in nursing (pp. 81-92). London: Grune \& Stratton, Inc.

Reason, P., \& Rowan, J. (Eds.). (1981). Human inquiry. New York: John Wiley \& Sons.

Reavy, K. (2003). Service learning: A case-study examining positive and negative influences on higher education faculty. (Doctoral dissertation, The 
University of Utah, 2003). Dissertation Abstracts International, 64, 08A, 2807

Redman, W., \& Clark, L. (2002). Service-learning as a model for integrating social justice in the nursing curriculum. Journal of Nursing Education, 41(10), 446449.

Reeb, R. (1994). The other's world. Nursing and Health Care, 15(5), 230.

Reed-Danahay (Ed.). (1997). Auto/Ethnography. New York: Berg.

Rinne, C. (1987). The affective domain--equal opportunity in nursing education? Journal of Continuing Nursing Education, 18(2), 40-43.

Roberts, C., Edwards, R., \& Barker, L. (1987). Intrapersonal communication processes. Scottsdale, Arizona: Gorsuch, Scarisbrick.

Roberts, C., Watson, K., \& Barker, L. (Eds.). (1989). Intrapersonal communication processes: Original essays. New Orleans: SPECTRA.

Rogo, K. (2004). Improving technologies to reduce abortion-related morbidity and mortality. International Journal of Gynecology \& Obstetrics, 85(Suppl. 1), S73-S82.

Roland, A. (1984). The self in India and America: Towards a psychoanalysis of social and cultural contexts. In V. Kavolis (Ed.), Designs of selfhood (pp. 171- 172). London: Associated University Presses.

Rolls, C., Inglis, A., \& Kristy, S. (1997). Study abroad programs: Creating awareness of and changing attitudes to nursing, health and ways of living in other cultures. Contemporary Nurse, 6(3/4), 152-156.

Rosaldo, R. (1989). Culture and truth: The remaking of social analysis. Boston: Beacon Press.

Rosenfield, A., \& Schwartz, K. (2005). Improving the health of women in developing countries: The time is now. Journal of Midwifery \& Women's Health, 50(4).

Ross, B. (1991). Remembering the personal past. New York: Oxford University Press.

Rountree, K., \& Laing, T. (1996). Writing by degrees. Auckland: Addison Wesley Longman New Zealand. 
Rowan, J. (1983). The real self and mystical experiences. Journal of Humanistic Psychology, 23(2), 9-27.

Royston, E., \& Armstrong, S. (1989). Preventing maternal deaths. Geneva: World Health Organization.

Ryan, M., Twibell, R., Brigham, C., \& Bennett, P. (2000). Learning to care for clients in their world, not mine. Journal of Nursing Education, 39(9), 401408.

Sachs, J., \& McArthur, J. (2005). The millennium project: A plan for meeting the millennium development goals. The Lancet, 365(9458), 347-353.

Salk, J. (1983). Anatomy of reality. New York: Columbia University Press.

Sandelowski, M. (1986). The problem of rigor in qualitative research. Advances in Nursing Science, 8(3), 27-37.

Sandin, I., Grahn, K., \& Kronvall, E. (2004). Outcomes of Swedish nursing students' field experiences in a hospital in Tanzania. Journal of Cultural Diversity, 15(3), 225-230.

Sargent, C., \& Brettell, C. (Eds.). (1996). Gender and health: An international perspective. Upper Saddle River: Prentice Hall.

Savage, J. (2004). Researching emotion: The need for coherence between focus, theory and methodology. Nursing Inquiry, 11(1), 25-34.

Scanlan, J. (1996). Clinical teaching: The development of expertise (symbolic interactionism). (Doctoral dissertation, University of Manitoba, Canada 1996). Dissertation Abstracts International, 58, 04B, 1807.

Schim, S. (2005). A picture on the front of the box. Journal of Professional Nursing, 21(5), 255-256.

Schim, S., Doorenbos, A., \& Borse, N. (2005). Cultural competence among Ontario and Michigan healthcare providers. Journal of Nursing Scholarship, Fourth quarter, 354-360.

Scholes, J., \& Moore, D. (2000). Clinical exchange: One model to achieve culturally sensitive care. Nursing Inquiry, 7(1), 61-71.

Schön, D. (1983). The reflective practitioner. New York: Basic Books. 
Schreiber, R. (2001). The "how to" of grounded theory: avoiding the pitfalls. In R. Scheiber \& P. N. Stern (Eds.), Using grounded theory in nursing (pp. 55-84). New York: Springer.

Schreiber, R., \& Stern, P. N. (2001). Introduction. In R. Schreiber \& P. N. Stern (Eds.), Using grounded theory in nursing (pp. xvi-xviii). New York: Springer.

Schreiber, R., \& Stern, P. N. (Eds.). (2001). Using grounded theory in nursing. New York: Springer.

Schultz, P. (2002). Why governments should invest more to educate girls. World Development, 30(2), 207-225.

Self, S. (2005). What makes motherhood so expensive? The role of social expectations, interdependence, and coordination failure in explaining lower wages of mothers. The Journal of Socio-Economics, 34, 850-865.

Shacklock, G., \& Smyth, J. (Eds.). (1998). Being reflexive in critical educational and social research (Vol. 18). London: Falmer Press.

Singh, R. R. (1991). Education for the twenty-first century: Asia-Pacific perspectives. Bangkok: UNESCO Regional Office.

Sloss, E., Mirsky, J., \& Radlett, M. (1998). Women's Health: Using human rights to gain reproductive rights. Retrieved 14 January, 2002

Smyth, J., \& Shacklock, G. (1998). Re-making teaching. New York: Routledge.

Smyth, W. (1986). Reflection-in-action. Victoria: Deakin University Press.

Sommer, R., \& Osmond, H. (1960). Autobiographies of former mental patients. Journal of Mental Science, 107, 1030-1032.

Spinelli, E. (1989). The interpreted world: An introduction to phenomenological psychology. Newbury Park, California: Sage.

Spradley, J. (1979). The ethnographic interview. New York: Holt, Rinhart, and Winston.

Spradley, J., \& McCurdy, D. (1972). The cultural experience. Chicago: Science Research Associates.

St. Clair, A., \& McKenry, L. (1999). Preparing culturally competent practitioners. Jounal of Nursing Education, 38, 228-234. 
State of world's children. (2001). The state of the world's children. New York: UNICEF.

Staunton, I. (1990). Mothers of the revolution. Bloomington: Indiana University Press.

Stearns, C., \& Stearns, P. (1986). Anger: The struggle for emotional control in America's history. Chicago: University of Chicago Press.

Stern, P. N. (2001). The "how to" of grounded theory": Avoiding the pitfalls. In R. Schreiber \& P. Stern (Eds.), Using grounded theory in nursing (pp. 55-84). New York: Springer.

Stevens, G. (1998). Experience the culture. Journal of Nursing Education, 37(1), 3033.

Stevens, P. (1989). A critical social reconceptualization of environment in nursing: Implications for methodology. Advances in Nursing Science, 11(4), 56-68.

Street, A. (1990). Nursing practice. Geelong, Victoria: Deakin University Press.

Street, A. (1992). Inside nursing. Albany: State University of New York Press.

Street, A. (1995). Nursing replay. Melbourne: Churchill Livingstone.

Streubert, H., \& Carpenter, D. (1999). Qualitative Research in Nursing (2nd ed.). Philapdelphia: Lippincott.

Study Abroad Program: Chancellor's office, Executive orders 550 and 590. Office of International Programs. California State University, Long Beach, California, (1985).

Subramanian, S., Chen, J., Rehkopf, D., Waterman, P., \& Kreiger, N. (2005). Racial disparities in context: A multilevel analysis of neighborhood variations in poverty and excess mortality among black population in Massachusetts. American Journal of Public Health, 95(2), 260-265.

Suominen, T., Kovasin, M., \& Ketola, O. (1997). Nursing culture-some viewpoints. Journal of Advanced Nursing, 25, 186-190.

Sutich, A., \& Vich, M. (Eds.). (1969). Readings in humanistic psychology. London: Collier-Macmillan Limited.

Swenson, D., \& Sims, S. (2003). Listening to learn: Narrative strategies and interpretive practices in clinical education. In N. Diekelmann (Ed.), Teaching 
the practitioners of care (pp. 154-193). Madison Wisconsin: The University of Wisconsin Press.

Szwarcwald, C., Tavares de Andrade, C., \& Bastos, F. (2002). Income inequality, residential poverty clustering and infant mortality: a study in Rio de Janeiro, Brazil. Social Science \& Medicine.

Tabi, M., \& Mukherjee, S. (2003). Nursing in a global community: A study abroad program. Journal of Transcultural Nursing, 14(2), 134-138.

Takacs, D. (2003). How does you positionality bias your epistemology. Thought and Action, 19 (1), 27-38.

Tamasese, K. (1998). O le faa'aloalo I le faa'aloalo. Dulwich Centre Newsletter (1), 34-36.

Tanner, C. (2004). The meaning of curriculum: Content to be covered or stories to be heard? Journal of Nursing Education, 43(1), 3-4.

Taylor, B. (2000). Reflective practice. St Leonards: Allen \& Unwin.

Taylor, S., \& Bogdan, R. (1984). Introduction to qualitative research methods (2nd ed.). New York: John Wiley \& Sons, Inc.

Thayer-Bacon, B. (2000). Transforming critical thinking. New York: Teachers College Press.

Thompson, K., Boore, J., \& Deeny, P. (2000). A comparison of an international experience for nursing students in developed and developing countries. International Journal of Nursing Studies, 37, 481-492.

Thoreau, H. D. (1854 /1985). A week on the Concord and Merrimack rivers. Walden: or, life in the woods. New York: Penguin Putnam.

Tinker, A. (2000). Women's health: the unfinished agenda. International Journal of Gynecology \& Obstetrics, 70, 149-158.

Tinkle, M., \& Beaton, L. (1983). Toward a new view of science: Implications for nursing research. Advances in nursing science, 5(2), 27-36.

Toms, J., \& Toms, M. (2003). New Dimensions (Radio broadcast). Washington, D. C.: National Public Radio.

Towards evidence. (2001). Towards evidence to secure reproductive rights. The Lancet, 363(9402), 1. 
Tsu, V. D., \& Shane, B. (2004). New and underutilized technologies to reduce maternal mortality: call to action from a Bellagio workshop. International Journal of Gynecology and Obstetrics, 85(Supp 1), 83-93.

Turabian, K. (1987). A manual for writers of term papers, theses and dissertations (5th ed.). Chicago: University of Chicago Press.

Turner, R., \& Short, J. (Eds.). (1984). Annual review of sociology (Vol. 10). Palo Alto, California: Annual Reviews.

UNICEF. (1999). Ujeli: A child bride in Nepal [video]. (Available from UNICEF: P.O. Box \#1187 Pulchowk, Kathmandu, Nepal).

Unterhalter, E. (2005). Global inequality, capabilities, social justice: The millennium development goal for gender equality in education. International Journal of Educational Development, 25, 111-122.

Van Manen, M. (1990). Researching lived experience. Ontario, Canada: Althouse Press.

Villarruel, A. (2004). Health disparities research: Issues, strategies, and innovations. The Journal of Multicultural Nursing and Health, 10(2), 7-12.

Vlassoff, C. (1994). Gender inequalities in health in the third world: Uncharted ground. Social Science \& Medicine, 39(9), 1249-1259.

Vocate, D. (1994). Self-talk and inner speech: understanding the uniquely human aspects of intrapersonal communication. In D. Vocate (Ed.), Intrapersonal communication: Different voices, different minds (pp. 3-32). Hillsdale: Lawrence Erlbaum Associates.

Vocate, D. (Ed.). (1994). Intrapersonal communication: Different voices, different minds. Hillsdale: Lawrence Erlbaum Associates.

Vygotsky, L. (1926/1997). Educational psychology (R. Silverman, Trans.). Boca Raton, Florida: St. Lucie Press.

Wagaarachchi, P., \& Lakshman, F. (2002). Trends in maternal mortality and assessment of substandard care in a tertiary care hospital. European Journal of Obstetrics \& Gynecology and Reproductive Biology, 101, 36-40. 
Waldegrave, C. (1999). Research and the public debate. Paper presented at the "A decade of change conference: Consequences for health" Health Research Council Conference, Wellington, New Zealand.

Waldegrave, C. (2000). "Just therapy" with families and communities. In G. Burford \& J. Hudson (Eds.), Family group conferencing (pp. 153-163). New York: Aldine De Gruyter.

Walsh, L. (2003). International service learning in midwifery and nursing education. Journal of Midwifery \& Women's Health, 48(6), 449-458.

Walsh, L., \& DeJoseph, J. (2003). I saw it in a different light: Learning experiences in baccalaureate nursing education. Journal of Nursing Education, 42(6), 266-272.

Warner, J. (2002). Cultural competence immersion experiences. Nurse Educator, 27(4), 187-190.

Warren, R. (2002). The purpose driven life. Grand Rapids, Michigan: Zondervan.

Watson, G. (1987). Writing a thesis. London: Longman.

Watson, J. (1985). Science of human caring. New York: National League for Nursing.

Watson, L., \& Watson-Franke, M. (1985). Interpreting life histories. New Brunswick: Rutgers University Press.

Watt, S., Law, K., Ots, U., \& Waagø, K. (2002). Reflections across boundaries: The European nursing module. Journal of Transcultural Nursing, 13(4), 318-324.

Weinsheimer, J. (1985). Gadamer's Hermeneutics: A reading of truth and method. London: Yale University Press.

Whiteford, L. (1996). Political economy, gender, and the social production of health and illness. In C. Sargent \& C. Brettell (Eds.), Gender and health (pp. 242259). Upper Saddle River: Prentice Hall.

Williams, C., Baumslag, N., \& Jelliffe, D. (1994). Mother and child health (3rd ed.). New York: Oxford University Press.

Williams, D. (2002). Racial/ethnic variations in women's health: The social embeddedness of health. Public Health Matters, 92(4), 588-597. 
Williamson, J. (1992). 'I peel myself out of my own skin': Reading don't: A woman's word. In M. Kadar (Ed.), Essays on life writing: from genre to critical practice (pp. 133-151). Toronto: University of Toronto Press.

Wolf, D. (1996). Situating feminist dilemmas in fieldwork. In D. Wolf (Ed.), Feminist dilemmas in fieldwork (pp. 1-55). Boulder, Colorado: Westview Press.

Wolf, D. (Ed.). (1996). Feminist dilemmas in fieldwork. Boulder, Colorado: Westview Press.

Wood, C. (2002b). Lift every voice. In S. Intrator (Ed.), Stories of the courage to teach (pp. 192-201). San Francisco: Jossey-Bass.

Wood, P. (2002a). Nursing's background of scholarly inquiry. In E. Papps (Ed.), Nursing in New Zealand: Critical issues, Different perspectives. Auckland: Pearson Education New Zealand Limited.

Yin, R. (2003). Case study research (3rd ed.). Thousand Oaks: Sage Publications.

Yonge, O. (1997). Assessing and preparing students for distance preceptorship placements. Journal of Advanced Nursing, 26, 812-816.

Zencey, E. (1995). Panama. New York: Berkley.

Zorn, C. (1996). The long-term impact on nursing students of participating in international education. Journal of Professional Nursing, 12(2), 106-110.

Zorn, C., Ponick, D., \& Peck, S. (1995). An analysis of the impact of participation in an international study program on the cognitive development of senior baccalaureate nursing students. Journal of Nursing Education, 34 (February), 67-70. 